\title{
Interplay of Verticillium signaling genes favoring beneficial or detrimental outcomes in interactions with plant hosts
}

\author{
Dissertation \\ For the award of the degree \\ "Doctor rerum naturalium" \\ of the University of Goettingen \\ within the doctoral program \\ "Plant Responses To Eliminate Critical Threats" \\ of the Georg-August University School of Science (GAUSS)
}

\author{
submitted by \\ Jessica Starke \\ from Celle
}

May 2019 



\section{Thesis Committee and members of the Examination Board}

Referee: Prof. Dr. Gerhard H. Braus

Department of Molecular Microbiology and Genetics

University of Goettingen

$2^{\text {nd }}$ referee: apl. Prof. Dr. Kai Heimel

Department of Molecular Microbiology and Genetics

University of Goettingen

$3^{\text {rd }}$ referee: Prof. Ph.D. James W. Kronstad

Michael Smith Laboratories

University of British Columbia Vancouver

Prof. Dr. Andrea Polle

Department of Forest Botany and Tree Physiology

University of Goettingen

Prof. Dr. Petr Karlovsky

Department of Molecular Phytopathology and Mycotoxin Research

University of Goettingen

Prof. Dr. Volker Lipka

Department of Plant Cell Biology

University of Goettingen

Date of oral examination: July $22^{\text {nd }} 2019$ 


\section{Declaration of independence}

Herewith I declare that the dissertation entitled "Interplay of Verticillium signaling genes favoring beneficial or detrimental outcomes in interactions with plant hosts" was written on my own and independently without any other aids and sources than indicated.

Jessica Starke

Goettingen, 2019 
This work was accomplished in the group of Prof. Dr. Gerhard H. Braus, at the Department of Molecular Microbiology and Genetics at the Institute of Microbiology and Genetics, University of Goettingen.

Parts of this work will be published in:

Harting $\mathrm{R}^{\star}$, Starke $\mathrm{J}^{\star}$, Kusch $\mathrm{H}$, Pöggeler S, Maurus I, Schlüter R, Landesfeind M, Bulla I, Nowrousian M, de Jonge R, Stahlhut G, Hoff K, Aßhauer KP, Thürmer A, Stanke M, Daniel R, Morgenstern B, Thomma BPHJ, Kronstad JW, Braus-Stromeyer SA, Braus GH (2019) A $20 \mathrm{~kb}$ Lineage Specific genomic region tames virulence in pathogenic allodiploid Verticillium longisporum. Manuscript in preparation. ( ${ }^{*}$ contributed equally)

Starke J, Harting R, Maurus I, Bremenkamp R, Kronstad JW, Braus GH (2020) Unfolded protein response and scaffold independent pheromone MAP kinase signalling control Verticillium dahliae growth, development and plant pathogenesis. BioRxiv 941450. doi:10.1101/2020.02.10.941450. 


\section{Table of Contents}

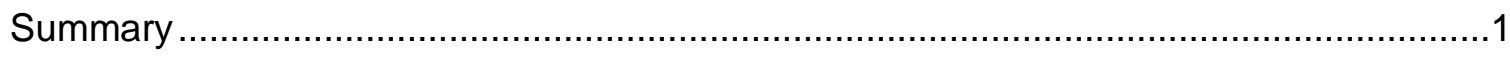

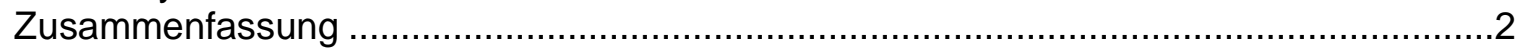

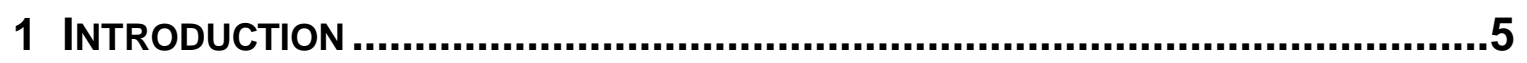

$1.1 \quad$ Verticillium spp. - colonizers of the plant's vascular system..............................

1.1.1 The ascomycete genus Verticillium ........................................................

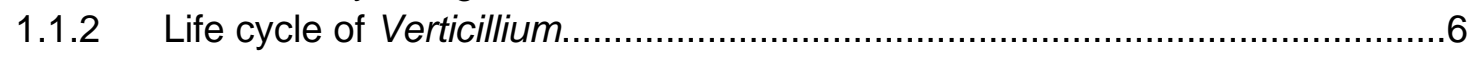

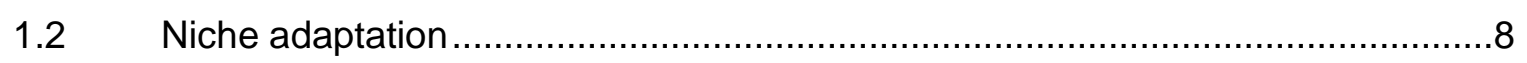

1.2.1 Verticillium dahliae Lineage Specific regions ..........................................

1.2.2 Interspecific hybridization of Verticillium longisporum ..................................10

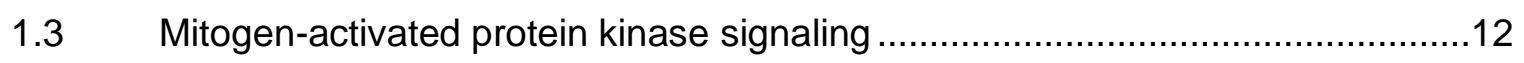

1.3.1 Fus3 and Kss1 MAPK signaling in Saccharomyces cerevisiae.....................13

1.3.2 Scaffolds in Fus3/Kss1 MAPK signaling in filamentous ascomycetes ..........15

1.3.3 Fus3/Kss1 MAPK signaling in plant pathogenic fungi..................................15

$1.4 \quad$ The unfolded protein response .................................................................... 17

1.4.1 The unfolded protein response pathway in Saccharomyces cerevisiae .........17

1.4.2 The unfolded protein response pathway in pathogenic fungi ........................19

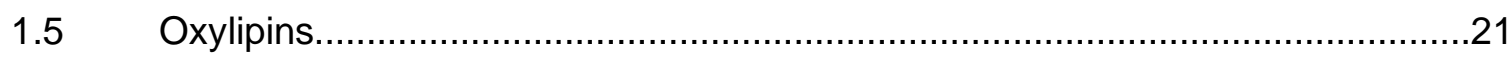

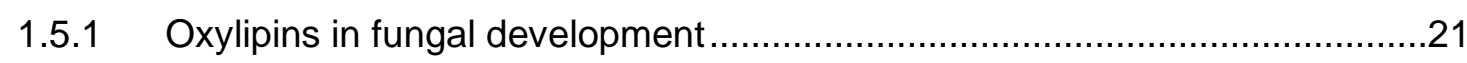

1.5.2 Oxylipins in plant-fungus interactions ......................................................23

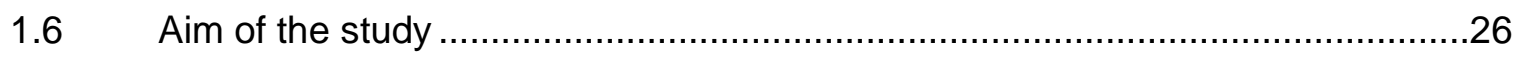

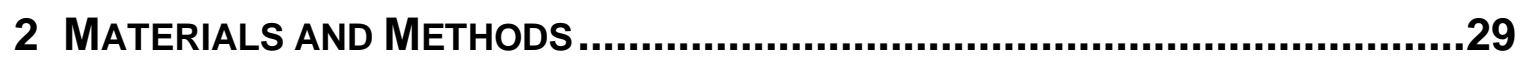

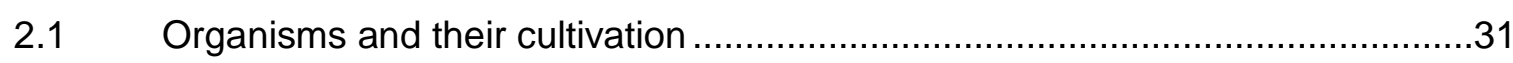

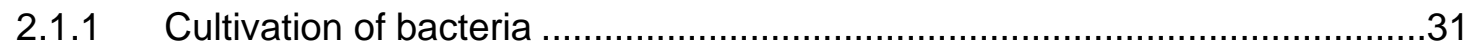

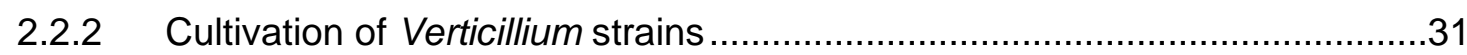

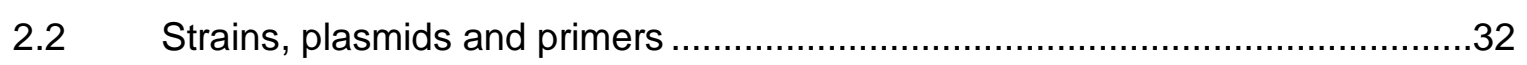

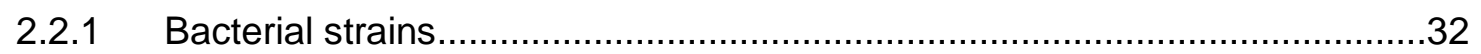

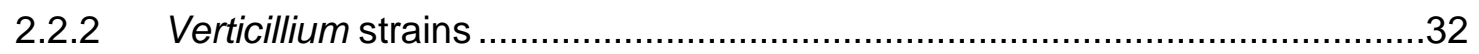

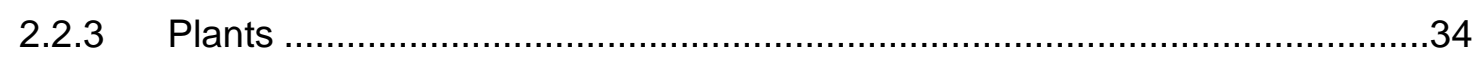

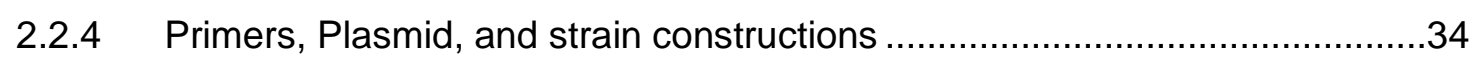

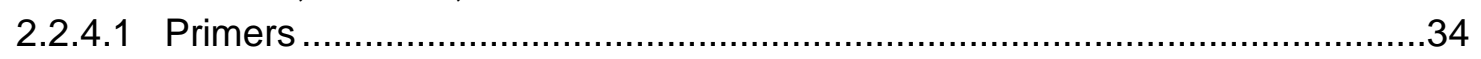

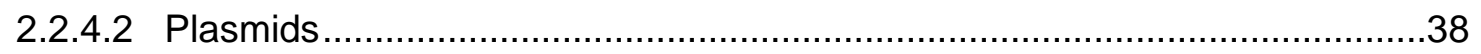

2.2.4.3 Plasmid and strain construction of $V$. longisporum strains ...........................40

2.2.4.4 Plasmid and strain construction of $V$. dahliae strains ..................................4 41

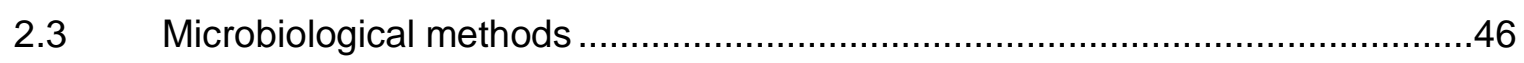

2.3.1 Transformation of Escherichia coli ...............................................................46

2.3.2 Transformation of Agrobacterium tumefaciens ...........................................46

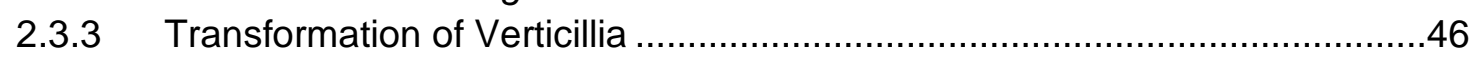

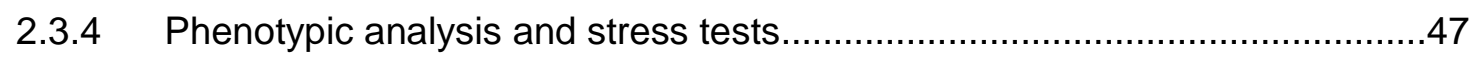

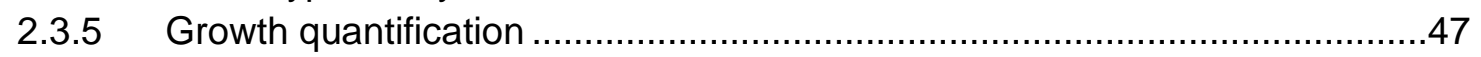

2.3.6 Microsclerotia observation and quantification of melanization.......................47

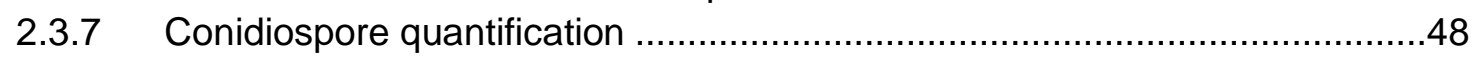

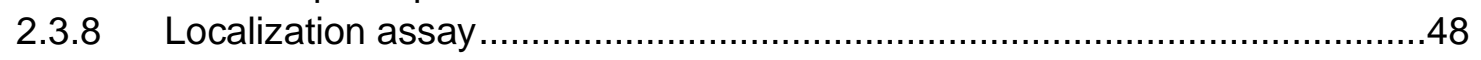

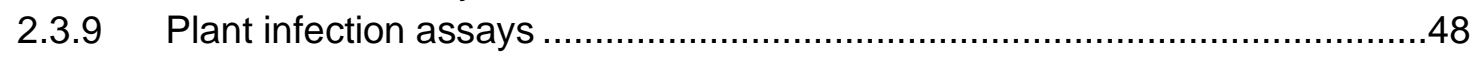


2.3.9.1 Brassica napus infection assay with $V$. longisporum .................................48

2.3.9.2 Solanum lycopersicum infection assay with $V$. dahliae................................49

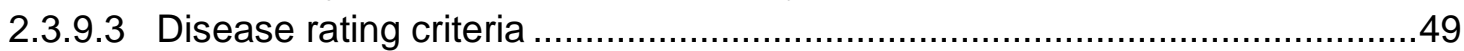

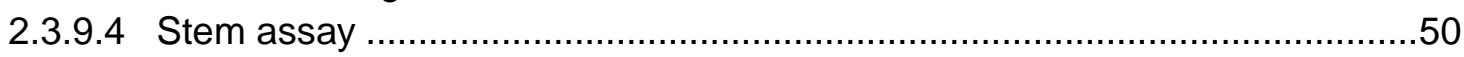

2.3.9.5 Arabidopsis thaliana root colonization assay ...........................................

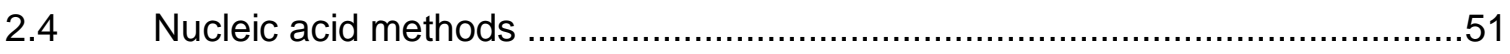

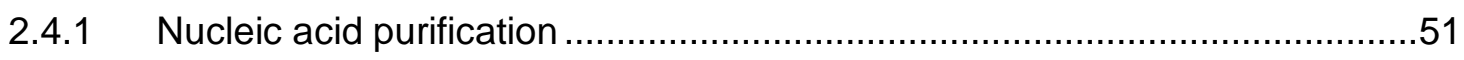

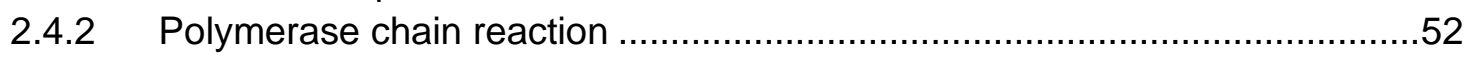

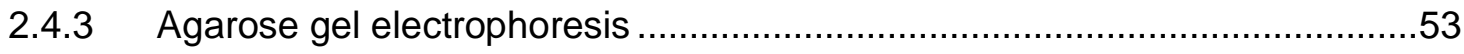

2.4.4 Abscence confirmation of the VI43LS2Okb region in $V$. longisporum $V I 32 \ldots . .53$

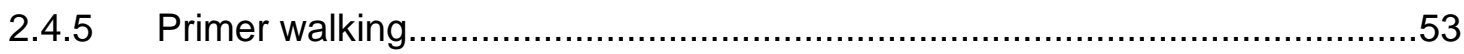

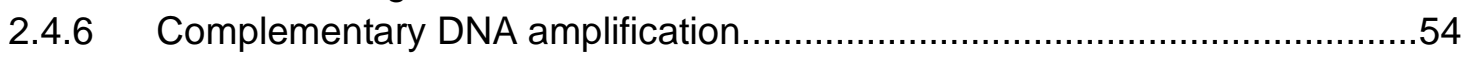

2.4.7 Quantitative reverse transcriptase PCR ..................................................54

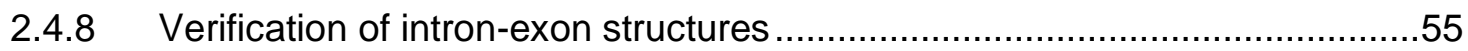

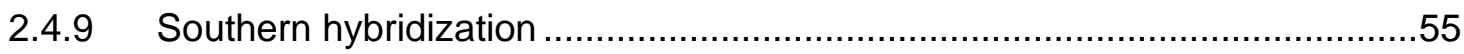

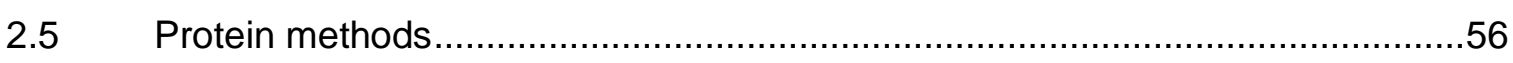

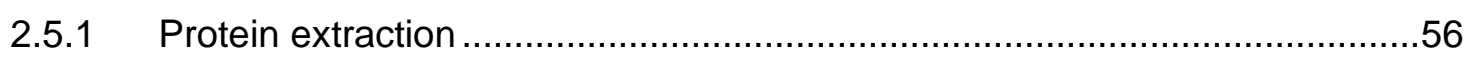

2.5.2 Determination of protein concentration by Bradford assay ...........................56

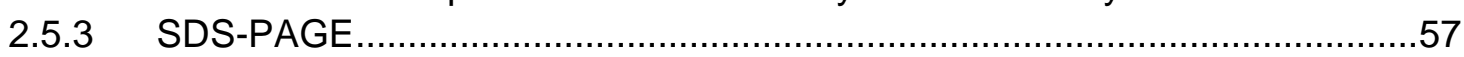

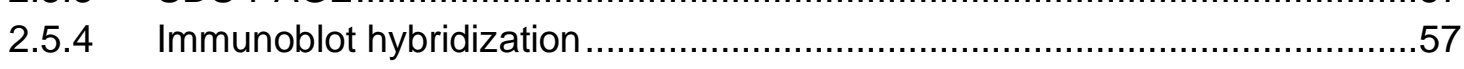

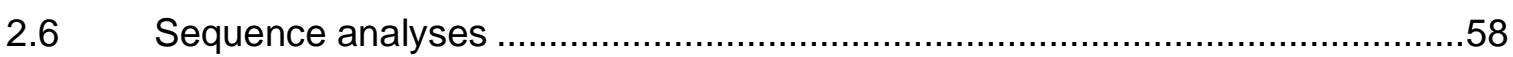

3 RESULTS ...........................................................................................59

3.1 The $V$. longisporum VI43LS20kb region makes the pathogenic isolate VI43 more tolerable for the plant and is absent in the asymptomatic isolate $\mathrm{VI} 32$.

3.1.1 V. longisporum strains VI43 and VI32 are able to colonize rapeseed plants with different consequences for plant growth and development .........59

3.1.2 The pathogenic $V$. longisporum VI43 genome harbors the VI43LS20kb region, which is absent in the asymptomatic colonizer VI32 ........................61

3.1.3 The VI43LS20kb region reduces virulence in rapeseed plants......................64

3.1.4 The homologous VI43LS20kb region in V. dahliae is indifferent for virulence towards tomato plants

3.2 Vmk1/Mek2 MAPK cascade-mediated microsclerotia formation and pathogenicity in $V$. dahliae are independent from the scaffold protein Ham5 ....67

3.2.1 V. dahliae Ham5 corresponds to the $N$. crassa scaffold protein HAM-5 .......67

3.2.2 The $V$. dahliae MAP2K Mek2 and the MAPK Vmk1 show high similarities to $N$. crassa MEK-2 and MAK-2...

3.2.3 Vmk1 - and Mek2-mediated regulation of vegetative growth and microsclerotia formation is independent from Ham5.

3.2.4 Mek2- and Vmk1-mediated regulation of virulence is independent from Ham5 in tomato plants.

3.3 The unfolded protein response pathway regulator Hac1 controls

development and virulence of $V$. dahliae ......................................................76

3.3.1 HAC1 and IRE1 genes of the UPR pathway in $V$. dahliae............................76

3.3.2 The HAC1 mRNA is unconventionally spliced in $V$. dahliae ..........................77

3.3.3 The unconventionally spliced mRNA variant $H A C 1^{i}$ is translated into the Hac1 protein in V. dahliae...

3.3.4 HAC1 is essential for microsclerotia formation of $V$. dahliae .........................82 
3.3.5 V. dahliae HAC1 is dispensable for penetration of the

A. thaliana root cortex

3.3.6 V. dahliae HAC1 is required for colonization of tomato plants.......................87

3.4 The $V$. dahliae oleate $\Delta 12$-fatty acid desaturase Ode1 promotes

differentiation with only a minor impact on virulence

3.4.1 V. dahliae Ode1 corresponds to the A. nidulans oleate $\Delta 12$-fatty acid desaturase OdeA.

3.4.2 V. dahliae Ode1 localizes to plasma membranes and cell organelle membranes

3.4.3 ODE1 is required for vegetative growth and microsclerotia formation in V. dahliae.

3.4.4 V. dahliae ODE1 is dispensable for induction of disease symptoms in tomato plants.

4.1 The pathogenic $V$. longisporum isolate VI43 possesses a genomic insertion contributing to disease symptom attenuation in rapeseed plants

4.1.1 V. longisporum isolates colonize plants with different pathotypes.................99

4.1.2 The $V$. longisporum VI43LS20kb region mediates virulence attenuation towards rapeseed plants........................................................................... 100

4.1.3 Host specificity of virulence and putative attenuating factors in Verticillia.. 104

4.2 MAPK signaling pathways in $V$. dahliae mediate differentiation, stress response and virulence independent from the scaffold protein Ham5

4.2.1 The Ham5 scaffold function for the Fus3/Kss1-like MAPK cascade is not conserved among filamentous ascomycetes.

4.2.2 V. dahliae Mek2 and Vmk1 are involved in fungal propagation in planta... 111

4.3 The unfolded protein response regulator Hac1 mediates differentiation and virulence in $V$. dahliae

4.3.1 Unconventional splicing and translation of the bZIP transcription factor Hac1 is conserved in V. dahliae....

4.3.2 The unfolded protein response regulator Hac1 mediates species-specific differentiation in V. dahliae.

4.3.3 V. dahliae Hac1 is essential for fungal colonization of the host plant......... 118

4.4 The $V$. dahliae oleate $\Delta 12$-fatty acid desaturase Ode1 has important roles

in fungal growth and is dispensable for induction of disease symptoms

4.4.1 The $V$. dahliae oleate $\Delta 12$-fatty acid desaturase Ode1 contributes to fungal growth and differentiation

4.4.2 Plant-derived unsaturated fatty acids might replace products synthesized by $V$. dahliae Ode1 during host colonization.

References

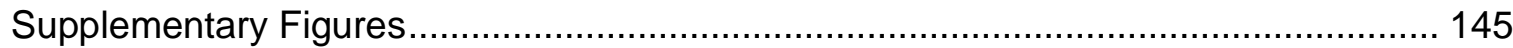

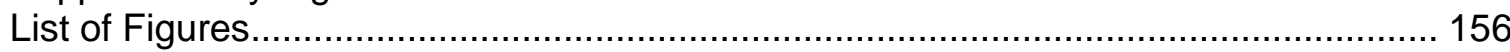

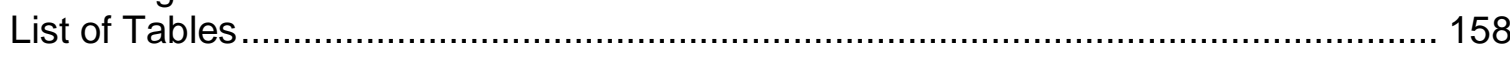

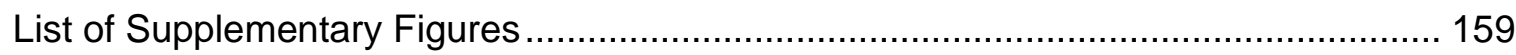

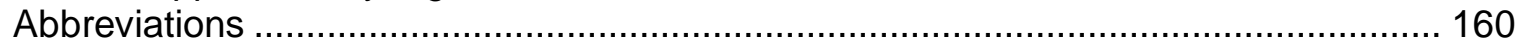

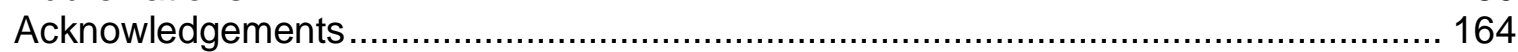

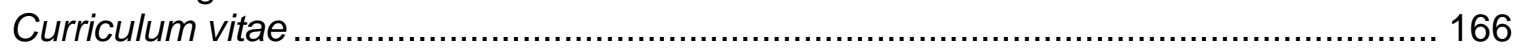




\section{Summary}

Verticillia and their plant hosts both contribute to pathogenesis as a consequence of their interaction. Evolution-driven genome extension includes hybridization, resulting in amphidiploid species as the rapeseed pathogen Verticillium longisporum and the acquirement of Lineage Specific (LS) regions in haploid Verticillia. Whether a fungus-plant interaction results in pathogenicity or reduced susceptibility of the host depends on mutual exchange of secreted signals, their perception and intracellular transduction pathways.

The genome of the haploid tomato pathogen $V$. dahliae JR2 includes an LS region with several genes for putative transcription factors, which is not required for virulence. Hybrid pathogenic V. longisporum isolates VI43 or VI145c contain the homologous VI43LS2Okb region, which is absent in the non-symptomatic hybrid VI32. Deletion of this region in $V$. longisporum VI43 led to a hypervirulent phenotype in rapeseed, suggesting a function in reducing virulence as well as susceptibility of the host plant.

The Fus3/Kss1-like $V$. dahliae MAP kinase Vmk1 plays an essential role in regulating virulence in plants. Intracellular transduction pathways can be insulated by scaffold proteins for maintenance of specificity. The $V$. dahliae MAPK scaffold protein Ham5, homologous to Neurospora crassa HAM-5, has not yet been analyzed in plant pathogens. Deletion of V. dahliae HAM5 allowed wild type-like development, whereas deletion of the upstream MAP2K MEK2 reduced vegetative growth and microsclerotia formation as deletion of VMK1. $M E K 2$ and $V M K 1$ deletion strains were re-isolated from plant tissue, supporting their potential to invade tomato plants. Both displayed defects in induction of disease symptoms. HAM5 deletion strains induced wild type-like disease symptoms in planta. Therefore, the Vmk1/Mek2 MAPK signaling pathway-mediated regulation of vegetative growth, microsclerotia formation and pathogenicity is independent from the scaffold protein Ham5 and does not require its insulation function in $V$. dahliae.

Plant invasion and immune suppression require secretion of correctly folded proteins monitored by the Hac1-controlled unfolded protein response (UPR) pathway. The bZIP transcription factor Hac1 is essential for virulence of the necrotrophic appressoria-forming ascomycete Alternaria brassicicola or of the basidiomycete Ustilago maydis. V. dahliae $H A C 1$ mRNA appears in two splice variants $H A C 1^{u}$ (uninduced) and $H A C 1^{i}$ (induced), where only $H A C 1^{i}$ was translated into a protein. $H A C 1$ deletion caused significantly decreased vegetative growth, conidiation and impaired microsclerotia formation. $\triangle H A C 1$ could penetrate and initiate colonization of the root cortex of Arabidopsis thaliana, but induced only minor disease symptoms in tomato plants. Different to the MAPK deletion strains, $\triangle H A C 1$ could not be re-isolated from plant stems, corroborating deficiency in propagation within the plant. Constitutively active UPR led to increased microsclerotia formation and induced strong disease symptoms in planta. In conclusion, V. dahliae HAC1 is an important regulator of growth and differentiation with strong impact on virulence and susceptibility.

Virulence is connected to fungal hormones. Fungal oxylipins control differentiation processes, secondary metabolite production or manipulation of plant host defense responses. The Ode1 oleate $\Delta 12$-fatty acid desaturase is localized to intracellular membranes and catalyzes the oxidation of oleic acid to linoleic acid as major oxylipin precursor. $V$. dahliae ODE1 deletion resulted in decreased vegetative growth and microsclerotia formation. However, the defect in ODE1 had minor impact on fungal virulence. In summary, the outcome of a fungus-plant interaction as result of a complex interplay of virulence enhancing and reducing mechanisms was analyzed: Lineage Specific insertions can be beneficial for the plant, single enzymes for linoleic acid production as precursor of oxylipins can make a minor contribution, whereas Hac1-regulated UPR or the scaffoldindependent pheromone response MAPK pathway are crucial for pathogenicity. 


\section{Zusammenfassung}

Sowohl Verticillien als auch ihre Wirtspflanzen tragen zur Entwicklung von Krankheitssymptomen als Folge ihrer Interaktion bei. In haploiden Verticillien wurden Hybridisierungen zur Entstehung des amphidiploiden Rapspathogens Verticillium longisporum, und der Erwerb von artspezifischen Regionen (LS Regionen) als Mechanismen evolutions-getriebener Genomerweiterung beschrieben. $\mathrm{Ob}$ aus einer PilzPflanzeninteraktion Pathogenität oder eine reduzierte Anfälligkeit der Wirtspflanze resultiert, hängt vom gegenseitigen Austausch von sekretierten Signalen und deren Wahrnehmung, sowie von intrazellulären Transduktionswegen ab.

Im haploiden Genom des Tomatenpathogens V. dahliae JR2 befindet sich eine VI43LS20kbRegion, die für mögliche Transkriptionsfaktoren kodiert und nicht für die Virulenz benötigt wird. Die Genome der pathogenen, amphidiploiden V. longisporum Isolate VI43 und VI145c enthalten ebenfalls diese LS Region, die jedoch nicht im Genom des asymptomatischen Hybriden VI32 zu finden ist. Die Deletion dieser Region führte in V. longisporum VI43 zu verstärkten Krankheitssymptomen in Rapspflanzen, woraus eine Funktion in der Reduzierung der Virulenz oder der Anfälligkeit der Wirtspflanze hervorgeht.

Die Fus3/Kss1-ähnliche MAP Kinase Vmk1 spielt eine essentielle Rolle in der Regulation der Virulenz in Pflanzen in $V$. dahliae. Intrazelluläre Signaltransduktionswege können durch sogenannte Scaffold-Proteine isoliert werden um die Spezifität aufrecht zu erhalten. Das entsprechende Homolog zum Neurospora crassa MAP Kinase Scaffold-protein HAM-5 wurde bisher nicht in Pflanzenpathogenen beschrieben und die Rolle der vorgeschalteten Kinase Mek2 ist in Verticillien bisher nicht bekannt. Die Deletion von V. dahliae HAM5 erlaubte eine Wildtyp-ähnliche Entwicklung, wohingegen die Deletion von MEK2, sowie die Deletion von $V M K 1$, in reduziertem vegetativen Wachstum und verringerter Mikrosklerotienbildung resultierten. MEK2 und VMK1 Deletionsstämme konnten aus Pflanzengewebe re-isoliert werden, was ihr Potenzial in die Wirtspflanze einzudringen bestätigt. Jedoch zeigten beide Stämme Defekte in der Induktion von Krankheitssymptomen in Tomatenpflanzen. HAM5 Deletionsstämme induzierten Wildtyp-ähnliche Krankheitssymptome in der Pflanze. Somit ist die Regulation von vegetativem Wachstum, Mikrosklerotienbildung und Pathogenität, die durch den Vmk1/Mek2 MAPK-Signalweg vermittelt wird, in $V$. dahliae unabhängig von dem Scaffold-Protein Ham5 und erfordert dessen Isolationsfunktion nicht.

Das Eindringen in die Wirtspflanze und die Immunsuppression erfordert die Sekretion von korrekt gefalteten Proteinen, welche durch den Hac1-kontrollierten Signalweg als Antwort auf ungefaltete Proteine (UPR) überwacht wird. Der bZIP Transkriptionsfaktor Hac1 ist essentiell für die Virulenz des nekrotrophen Appressorien-bildenden Ascomyceten Alternaria brassicicola, sowie des Basidiomyceten Ustilago maydis. Die V. dahliae HAC1 mRNA kommt in zwei Spleißvarianten $H A C 1^{u}$ (uninduziert) und $H A C 1^{i}$ (induziert) vor. Jedoch wird nur $H A C 1^{i}$ in ein Protein übersetzt. Die Deletion von $H A C 1$ führte zu signifikant verringertem vegetativen Wachstum, verringerter Konidienbildung und zur Unfähigkeit Mikrosklerotien als Überdauerungsstrukturen zu bilden. $\triangle H A C 1$ konnte den Wurzelkortex von Arabidopsis thaliana-Pflanzen penetrieren und die Kolonisierung initialisieren, induzierte jedoch deutlich weniger starke Krankheitssymptome in Tomatenpflanzen. Anders als die MAP Kinase-Deletionsstämme konnte $\triangle H A C 1$ nicht aus dem Pflanzenmaterial re-isoliert werden. Dies spricht für einen Defekt in der Vermehrung des Pilzes in der Pflanze. Ein konstitutiv aktiver UPR Signalweg führte zu verstärkter Mikrosklerotienbildung und einer Induktion von starken Krankheitssymptomen in Tomatenpflanzen. Folglich spielt HAC1 eine entscheidende Rolle in der Regulation von Wachstum und Differenzierung, und wird für die Virulenz von $V$. dahliae und die Induktion von Krankheitssymptomen in der Pflanze benötigt. 
Des Weiteren gibt es Verbindungen zwischen Virulenz und pilzlichen Hormonen. Diese sogenannten Oxylipine können Differenzierungsprozesse, die Produktion von Sekundärmetaboliten und die Manipulation der Immunabwehr der Wirtspflanze beeinflussen. Die Ode1 Oleat- $\Delta 12$ Fettsäuredesaturase ist in intrazellulären Membranen lokalisiert und katalysiert die Oxidation von Ölsäure zu Linolsäure als Hauptvorläufer von Oxylipinen. Die Deletion des $V$. dahliae Gens ODE1 resultierte in verringertem vegetativem Wachstum und einer reduzierten Mikrosklerotienbildung. Allerdings zeigte der Defekt im ODE1 Gen nur einen geringen Einfluss auf die pilzliche Virulenz.

Zusammenfassend wurde der Erfolg einer Pilz-Pflanzeninteraktion als Ergebnis eines komplexen Zusammenspiels aus Virulenz-verstärkenden und -reduzierenden Mechanismen untersucht: Artspezifische Insertionen im Pilzgenom können vorteilhaft für die Wirtspflanze sein, einzelne Enzyme für die Produktion von Linolsäure als Vorläufer von Oxylipinen haben einen geringen Einfluss, während der Hac1-regulierte UPR Signalweg, sowie der Scaffoldunabhängige Pheromon-MAPK-Signalweg, eine entscheidende Rolle für die Pathogenität spielen. 


\section{INTRODUCTION}

\subsection{Verticillium spp. - colonizers of the plant's vascular system}

\subsubsection{The ascomycete genus Verticillium}

The plant disease Verticillium wilt is caused by members of the soil-borne ascomycete genus Verticillium, which contains ten species: V. albo-atrum sensu stricto, V. alfalfae, V. nonalfalfae, $\quad$ V.dahliae, $\quad$ V. isaacii, V. klebahnii, V. longisporum, V. nubilum, V. tricorpus and V. zaregamisanum (Inderbitzin et al., 2011a; Inderbitzin \& Subbarao, 2014). Conidiophores carry verticillate arranged phialides as conidia producing cells, giving the genus the name Verticillium (Pegg \& Brady, 2002). These species are distributed in temperate and subtropical regions world-wide and vary in their host ranges (Pegg, 1984; Fradin \& Thomma, 2006). The induced symptoms differ between hosts as well as between isolates from a certain species and may comprise stunting, chlorosis, necrosis, early senescence, defoliation, vein clearing, brownish vascular discoloration, or stem striping (Fradin \& Thomma, 2006; Depotter et al., 2016).

Verticillium species produce different dormant structures as resting mycelium, chlamydospores, or microsclerotia, which can persist in the soil (Wilhelm, 1955; Inderbitzin et al., 2011a; Carroll et al., 2018). Increasing temperatures due to climate change are assumed to increase the regional distribution and the economic impact of plant pathogens (Velásquez et al., 2018), like Verticillia, allowing more successful overwintering, extension of the growth season, and potentially earlier infection of younger plants. Furthermore, severity of disease symptoms induced by several Verticillium species was observed to be correlated with increasing temperatures (Koike et al., 1994; Jabnoun-Khiareddine et al., 2006; Siebold \& von Tiedemann, 2013). Available treatments for disease control include planting of resistant varieties, soil fumigation, and crop rotation with non-susceptible plants, but are costly, have questionable effects on the environment, or are not effective (Subbarao et al., 2007; Klosterman et al., 2009; Enebak et al., 2012; Carroll et al., 2018).

$V$. dahliae causes vascular wilting disease in almost 200 host species, including a broad range of crop plants and, therefore, has a great economic impact (Pegg \& Brady, 2002; Luo et al., 2014). Even if the species $V$. dahliae has a broad host range, single isolates of the species show altered aggressiveness on different plant hosts and can even colonize plants without induction of disease symptoms (Resende et al., 1994; Zeise \& von Tiedemann, 2002; Pegg \& Brady, 2002; Gibriel et al., 2019). Other members of the genus display narrower host ranges and distributions, however, significant economic losses are caused by $V$. albo-atrum, $V$. alfalfae, $V$. nonalfalfae, $V$. tricorpus, and 
V. zaregamsianum (Inderbitzin \& Subbarao, 2014). The species V. isaacii, V. klebahnii, and $V$. nubilum are pathogens with minor economic impact (Inderbitzin \& Subbarao, 2014). V. longisporum is the only interspecific hybrid of the genus with an amphidiploid genome (Ingram, 1968), mainly virulent on Brassicaceae (Zeise \& von Tiedemann, 2002; Eynck et al., 2007). Recently, preliminary data from field trials in the United Kingdom were published, suggesting inconsistent impact on yield reductions caused by rapeseed infections with $V$. longisporum despite the presence of disease symptoms (Depotter et al., 2019). The two species $V$. dahliae and $V$. longisporum are in scope of this study.

\subsubsection{Life cycle of Verticillium}

Species of the genus Verticillium induce Verticillium wilt, a monocyclic disease with one infection cycle per growing season (Fradin \& Thomma, 2006; Klosterman et al., 2011; Depotter et al., 2016a). V. dahliae and V. longisporum form thick-walled, black melanized microsclerotia from swollen, septate hyphae as a characteristic dormant structure (Griffiths, 1970; Pegg \& Brady, 2002; Figure 1, bottom). These resting structures persist in the soil through the winter or, if necessary, for up to 14 years (Wilhelm, 1955). Recognition of root exudates or plant surface molecules of an appropriate host is the first step in pathogen-plant communication, inducing germination of the fungus (Berlanger \& Powelson, 2000). The fungus initially colonizes the root surface and invades the plant via natural root wounds, or by development of swollen hyphal tips, which were described as penetration structures, named hyphopodia (Pegg \& Brady, 2002; Reusche et al., 2014; Zhao et al., 2014, 2016; Su et al., 2018; Figure 1, left). Root tips, wounds, and lateral root hairs are favored entry points (Fitzell et al., 1980; Eynck et al., 2007; Vallad \& Subbarao, 2008; Su et al., 2018).

From cortical cells hyphae migrate towards the vascular parenchyma and xylem vessels by intercellular growth and some of them successfully reach the xylem (Klosterman et al., 2009). Asexual spores are formed and spread within the vascular system via the transpiration stream (Klosterman et al., 2009). The size and shape of these conidia are the major morphological characteristic to distinguish between $V$. dahliae and $V$. longisporum. $V$. dahliae forms conidia of ovoid shape and a length between 3.5 to $5.5 \mu \mathrm{m}$, whereas the name $V$. longisporum hints to the formation of elongated conidia of 7.1 to $8.8 \mu \mathrm{m}$ (Karapapa et al., 1997; Collins et al., 2003; Tran et al., 2013; Figure 1, top).

A switch from the biotrophic to the necrotrophic life style corresponds with the colonization of tissues neighboring the xylem (Fradin \& Thomma, 2006). Disease symptoms induced by $V$. dahliae vary between fungal isolates and host plants and 
include stunting, chlorosis, necrosis, early senescence, defoliation, vein clearing, and brownish vascular discoloration (Fradin \& Thomma, 2006; Figure 1, right).

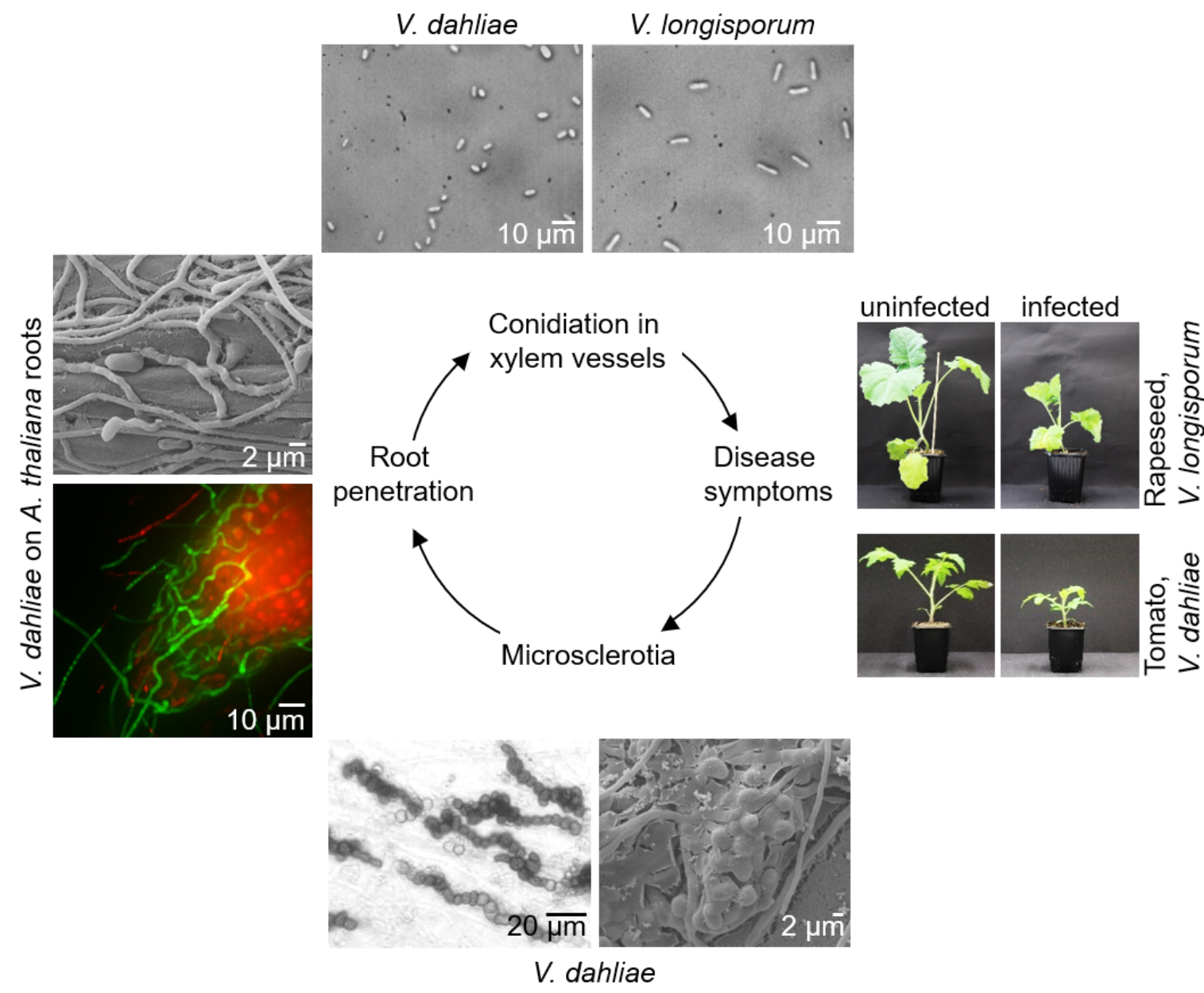

Figure 1: Verticillium life cycle. $V$. dahliae and $V$. longisporum form black melanized microsclerotia as dormant structures. In the presence of a suitable host the fungus germinates and grows into direction of the plant (Berlanger \& Powelson, 2000). It colonizes the root surface and invades root tips, lateral root hairs, or natural root wounds by intercellular growth or formation of hyphopodia (Fitzell et al., 1980; Eynck et al., 2007; Vallad \& Subbarao, 2008; Reusche et al., 2014; Zhao et al., 2014, 2016). Hyphae migrate from the cortex to the xylem vessels (Klosterman et al., 2009). Conidia are formed and spread within the vascular system with the transpiration stream (Klosterman et al., 2009). The fungus starts to colonize tissues neighboring the xylem with or without induction of disease symptoms. Under laboratory conditions the most obvious disease symptoms induced by $V$. longisporum in rapeseed plants or $V$. dahliae in tomato plants is stunting. In the dying host or under nutrient-limited conditions the fungus starts microsclerotia formation. Left: electron microscopy picture of $V$. dahliae growing on Arabidopsis thaliana roots (upper, Rabea Schlüter, Greifswald) and confocal fluorescence microscopy pictures from $V$. dahliae expressing ectopic GFP colonizing A.thaliana root tips stained in red with propidium iodide/silwet solution (lower). Top: V. dahliae forms smaller conidia (3.5 to $5.5 \mu \mathrm{m}$ ) with ovoid shape, whereas $V$. longisporum forms elongated conidia (7.1 to $8.8 \mu \mathrm{m})$. Right: Rapeseed plants 35 days after root dipping into distilled water or $V$. longisporum VI 43 spore solution (top). Tomato plants 21 days after root dipping into distilled water or V. dahliae JR2 spore solution (lower). Bottom: Microscopy picture of microsclerotia formed by $V$. dahliae nine days after spot inoculation of 50000 spores onto cellulose containing medium (left) and electron microscopy picture of microsclerotia formed by $V$. dahliae after four days on solid simulated xylem medium (Rabea Schlüter, Greifswald). 
V. longisporum-induced symptoms in rapeseed plants in the field are stem striping due to necrosis of the cortical tissue, whereas no wilting can be observed (Heale \& Karapapa, 1999; Depotter et al., 2016a). In contrast, artificial infection of rapeseed plants with pathogenic $V$. longisporum isolates by root dipping of seedlings results in stunting, chlorosis, necrosis, and increased branching (Zeise \& von Tiedemann, 2002; Eynck et al., 2007, 2009; Floerl et al., 2008; Depotter et al., 2016a; Figure 1, right). Verticillia are able to colonize plants without induction of disease symptoms. This can be observed either because the host is not susceptible, these plants are commonly considered as "non-host" or better as "asymptomatic host" plants (Malcolm et al., 2013), or because the Verticillium isolate is "non-pathogenic".

The fungus recognizes limited nutrient availability in the dying host plant or in plant debris, which induces the formation of resting structures (Fradin \& Thomma, 2006). These resting structures remain in the soil until perception of a new host. A sexual reproduction stage has not been described for any species of the genus (Short et al., 2014).

During all steps of the plant colonization cycle fungi perceive signals from the host environment, which induce differentiated development. On the other hand, the fungus sends signals like effectors to invade and suppress the plant immune system, which is required for successful colonization.

\subsection{Niche adaptation}

The fungus-host plant interaction is based on coevolution as a process of ongoing adaptation of both to changing external or internal environmental conditions. Success of the fungus requires to overcome the host's immune system, which evolves to limit the propagation of the invading fungus. Furthermore, fungi evolve to adapt to new host species with other defense mechanisms than previous hosts. Chromosomal recombination by meiosis has been seen as major evolutionary mechanism (Williams, 1975; de Jonge et al., 2013). However, in about $20 \%$ of all fungi, including Verticillia, no sexual stage has been observed (Heitman et al, 2007; Seidl \& Thomma, 2014). Evolution of clonally reproducing asexual fungi can include mechanisms as random mutations due to replication errors, horizontal gene or chromosome transfer, transposable elements, chromosomal rearrangements leading to deletions, duplications, inversions and translocations, and the hybridization of genomes (Brasier, 2000; Coleman et al., 2009; Klosterman et al., 2011; Ma et al., 2011; Mehrabi et al., 2011; Rouxel et al., 2011; de Jonge et al., 2012; Seidl \& Thomma, 2014; Faino et al., 2016). The genomes of some filamentous pathogens were grouped into a slowly evolving core genome, that maintains general physiology and a smaller, more quickly evolving pan genome with 
higher plasticity, enriched in genes for niche adaptation (Raffaele \& Kamoun, 2012; Faino et al., 2016). Regions representing the more flexible pan genome are also described as Lineage Specific (LS) regions, displaying the genomic regions of a lineage, that differentiates it from its relatives (Klosterman et al., 2011; Faino et al., 2016; Shikunne \& Faino, 2017). The impact of LS regions and genome hybridization on niche adaptation in Verticillia is one focus of this thesis.

\subsubsection{Verticillium dahliae Lineage Specific regions}

Lineage Specific genomic regions, unique or shared by a subset of strains, represent the pan genome as the part of the genome with higher plasticity (Klosterman et al., 2011; de Jonge et al., 2013). LS regions in $V$. dahliae were described to be acquired through horizontal gene transfer from Fusarium oxysporum (Chen et al., 2018) and plants (de Jonge et al., 2012), or transposons (Klosterman et al., 2011; Faino et al., 2016), and were observed to be correlated with synteny breakpoints arising from chromosomal reshuffling (de Jonge et al., 2013; Shi-kunne \& Faino, 2017). In Verticillia, generally frequent chromosomal rearrangements were observed in pathogenic and nonpathogenic species (Shi-kunne \& Faino, 2017). A correlation of synteny breakpoints with LS regions has not been analyzed in other Verticillia than $V$. dahliae yet (Shi-kunne \& Faino, 2017).

Research on LS regions to date focused especially on effector genes encoded in these regions. Effectors are typically small, cysteine-rich proteins with secretion signals, which are assumed to support colonization of the host by suppression of the plant immune response or manipulation of the host's cell physiology (Stergiopoulos \& de Wit, 2009; Selin et al., 2016). Gibriel et al. defined the relative proportion of the core and LS regions comparing $19 \mathrm{~V}$. dahliae strains with $93-97 \%$ core genome to 3-7\% LS regions (Gibriel et al., 2019). The analyzed core genomes displayed an average gene number of 9886 genes with 171 genes classified as effector genes by bioinformatical prediction. For the LS regions of these strains a total gene number ranging from 517 to 1318 was predicted, with an average effector gene number of 15 . Similar results were previously published for comparison of a smaller group of V. dahliae strains (de Jonge et al., 2013), suggesting that the relative number of genes important for niche adaptation is enriched in LS regions in $V$. dahliae. Focusing on bioinformatically predicted effector genes in LS regions, virulence factors have been identified in different $V$. dahliae strains (de Jonge et al., 2013; Faino et al., 2016; Kombrink et al., 2017; Li, 2019, Dissertation, University Wageningen). Examples that contribute to pathogenicity are the in planta induced effector Ave1 from a subset of $V$. dahliae strains (de Jonge et al., 2012; Faino et al., 2016; Song et al., 2018), the chitin-binding lysin motif effector LysM unique in VdLs.17 
(Kombrink et al., 2017), the defoliating pathotype-specific D effector (Li, 2019, Dissertation, University Wageningen), and the effector Sun1 (sunflower 1) specifically required for full pathogenicity of $V$. dahliae strains in sunflower infection ( $\mathrm{Li}, 2019$, Dissertation, University Wageningen). The effector protein Tom1 (tomato 1) was found to be essential for pathogenicity on tomato plants following identification of LS regions present in 11 tomato-infecting $V$. dahliae strains in comparison to six strains, which were avirulent on this host (Li, 2019, Dissertation, University Wageningen).

Even if the effector genes were found to be enriched in $V$. dahliae LS regions in comparison to the less flexible core genome, the number of effector genes relative to the total number of genes encoded in the LS regions is rather small (de Jonge et al., 2013; Gibriel et al., 2019). Different to the studies, comparing V. dahliae isolates, Klosterman et al. identified LS regions of $V$. dahliae by comparison to $V$. alfalfae genomes (formerly $V$. albo-atrum) and found the same relative numbers of secreted proteins in the core and LS regions (Klosterman et al., 2011). Besides transposable elements, the flexible regions contained gene duplications and genes, which were predicted to be potentially involved in pathotype specificity, signaling processes, transcriptional regulation, degradation of plant material, and iron or lipid metabolism (Klosterman et al., 2011). The role of LS genes in niche adaptation different to effector genes remains to be elucidated.

\subsubsection{Interspecific hybridization of Verticillium longisporum}

Genome evolution and niche adaptation through interspecific hybridization was described in several plant colonizing fungi, including the Brassicaceae colonizer V. longisporum (Clewes et al., 2008; Oberhofer \& Leuchtmann, 2012; Stukenbrock et al., 2012; Menardo et al., 2016). V. longisporum is the only interspecific hybrid of its genus with an amphidiploid genome (Clewes et al., 2008). The species evolved from at least three separate hybridization events from two haploid parental lineages (Inderbitzin et al., 2011b; Figure 2). Hybridization occurred for all known V. longisporum lineages between one unknown species named $A 1$, which is a close relative of $V$. alfalfae, and either one of two $V$. dahliae species named D2 and D3, or another unknown species named D1, as a close relative of $V$. dahliae (Inderbitzin et al., 2011b). The lineage A1/D2 has been exclusively detected in the USA, whereas the lineages $A 1 / D 1$ and $A 1 / D 3$ have been identified in Europe, Japan and the USA (Zeise \& von Tiedemann, 2002; Tran et al., 2013; Depotter et al., 2017). A1/D1 and A1/D3 lineages were found as coexisting species as, for example, the A1/D1 isolate VI43 and the A1/D3 isolate VI32 analyzed in this study, which were obtained from fields in the same area in Germany (Zeise \& von Tiedemann, 2002; Tran et al., 2013). The three hybrids display differences in pathogenicity towards different host plants, with lineages A1/D1 as the most pathogenic 
on rapeseed, A1/D2 as the most pathogenic on cabbage and horseradish, and lineage A1/D3 as the least pathogenic, but also found in rapeseed (Novakazi et al., 2015; Figure 2). Overall, V. longisporum lineages are mainly virulent on Brassicaceae (Zeise \& von Tiedemann, 2002; Eynck et al., 2007), whereas the haploid species $V$. dahliae and V. alfalfae cause disease in different hosts (Pegg \& Brady, 2002), but are less successful on Brassicaceae (Zhou et al., 2006). One theory is, that the increased fitness of $V$. longisporum on Brassicaceae in comparison to the parental lineages might have outcompeted the unknown parental lineages A1 and D1 (Depotter et al., 2016b).

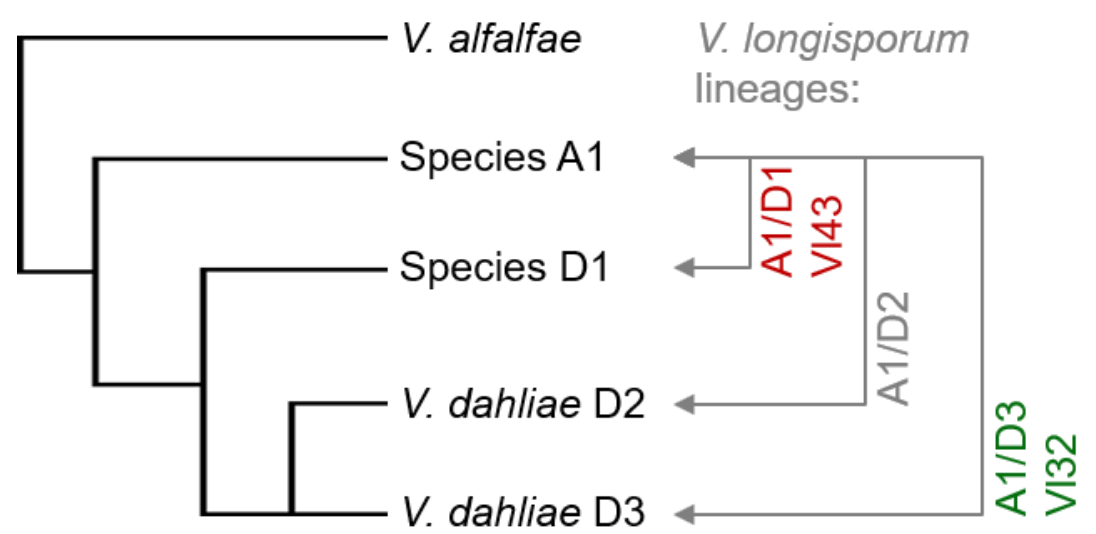

Figure 2: Evolution of $\boldsymbol{V}$. longisporum hybrids. The interspecific hybrids belonging to the species $V$. longisporum evolved at least three times by separate hybridization events, resulting in the lineages $A 1 / D 1, A 1 / D 2$, and $A 1 / D 3$. The species $A 1$ is an unknown species, which is phylogenetically closer to $V$. alfalfae. The species D1 is another unknown species closer related to $V$. dahliae. The parental lineages D2 and D3 are $V$. dahliae strains. The lineage A1/D2 has been found exclusively in the USA, whereas the lineages A1/D1 and A1/D3 have been identified in Europe, Japan and the USA (Depotter et al., 2017) and were found as coexisting species. The A1/D1 isolate VI43 and the A1/D3 isolate VI32 were obtained from fields in the same area in Germany and are studied in this work (Zeise \& von Tiedemann, 2002; Tran et al., 2013). Arrows indicate parental species of $V$. longisporum lineages. The tree is not scaled. (Modified from Inderbitzin \& Subbarao, 2014).

Mechanisms leading to hybridization are either sexual mating or the fusion of hyphae. Even if the prerequisites for sexual mating, including mating and meiosis-specific genes, are given in the genomes of Verticillia, a sexual reproduction stage has not been described for any species of the genus (Short et al., 2014; Milgroom et al., 2014). Opposite idiomorphs of the MAT locus, the major regulators of sexual recombination in ascomycetes, are required for sexual compatibility (Metzenberg \& Glass, 1990; Turgeon \& Yoder, 2000; Debuchy \& Turgeon, 2006). Most characterized V. longisporum isolates harbor copies of the MAT1-1 idiomorph in their genomes (Inderbitzin et al., 2011b; Depotter et al., 2017). Recently two isolates were described to contain two copies of the MAT1-1 idiomorph and one MAT1-2 idiomorph, each (Fogelqvist et al., 2018). In 
contrast, in a collection of $1120 \mathrm{~V}$. dahliae isolates the distribution of the mating type was skewed towards MAT1-2 among isolates with 99\% (Short et al., 2014). The observation of extensive sequence changes in the MAT loci and the absence of intermixing between genetic clusters in population structure analysis suggests that sexual reproduction of V. longisporum is unlikely (Depotter et al., 2017).

Another mechanism causing hybridization of genomes is parasexual reproduction by hyphal anastomosis followed by fusion of nuclei, which results in duplication of the parental genomes (Karapapa et al., 1997; Depotter et al., 2016b). Vegetative selfanastomoses of $V$. longisporum hyphae have been observed on specific media (unpublished data Rabea Schlüter, University of Greifswald). However, the observation of interspecific vegetative hyphal fusions of haploid Verticillia relies on auxotrophic mutants and respective selection in the laboratory and does not allow conclusions about the field situation (Hastie, 1973, 1989).

\subsection{Mitogen-activated protein kinase signaling}

As a result of niche adaptation conserved signaling pathways were shown to be modified in several species. This was also shown for mitogen-activated protein kinase (MAPK) signaling cascades. These cascades are activated by external signals enabling organisms to adapt to certain environmental conditions as ,for example, the germination and activation of infection-related programs following perception of a host plant. The core module of MAPK signaling pathways consists of a MAP kinase kinase kinase (MAP3K), a MAP kinase kinase (MAP2K), and a MAP kinase (MAPK) that sequentially phosphorylate one another and regulate downstream targets by phosphorylation. This module is highly conserved among eukaryotes from yeast to plants and mammals (Widmann et al., 1999). Different MAPK pathways share several components, like the MAP kinases, adaptor proteins or upstream kinases. Certain MAPK cascade scaffold proteins are necessary to bring the components of the MAPK module in proximity and maintain pathway specificity (Schaeffer \& Weber, 1999; Patterson et al., 2010). In Saccharomyces cerevisiae five MAPK cascades are described, which are defined by the name of the final MAPK: cell wall remodeling is mediated by the MAPK SIt2 (Suppressor of lytic phenotype), response to high osmolarity is achieved by Hog1 (High osmolarity glycerol response), Smk1 (Sporulation-specific mitogen-activated protein kinase) is required for sporulation, Kss1 (Kinase suppressor of Sst2 mutations) induces cell cycle arrest and invasive filamentous growth, and Fus3 (cell fusion 3) regulates mating as a response to pheromones (Herskowitz, 1995; Madhani \& Fink, 1998). Within ascomycetes homologs of three MAPK cascades, the Fus3/Kss1, Slt2 and Hog1 signaling pathways, can be found (Turrà et al., 2014). Most filamentous fungi have only 
one MAPK homologous to the yeast Fus3 and Kss1 MAPK (Jiang et al., 2018a). During this study the role of a potential scaffold protein for the yeast Fus3/Kss1-like MAPK pathway, known to be essential for pathogenicity of $V$. dahliae, was analyzed. Further, the MAP2K of the corresponding MAPK pathway was characterized. The MAPK signaling cascade is best described in S. cerevisiae and the nomenclature often refers to this organism.

\subsubsection{Fus3 and Kss1 MAPK signaling in Saccharomyces cerevisiae}

The yeast pheromone response MAPK pathway is activated upon pheromone binding to the guanine nucleotide-binding (G)-protein coupled membrane receptors Ste2/Ste3 (Sterile2/3), recruiting the scaffold protein Ste5 (Sterile5) to the membrane (Hamel et al., 2012; Figure 3A). The membrane-bound $\beta y$-subunits of the G-protein bind the kinase Ste20 (Sterile20) and the scaffold protein Ste5 to initiate signaling (Sabbagh et al., 2001). Ste20 phosphorylates the first kinase of the MAPK module Ste11 (Sterile11; Van Drogen et al., 2001). Ste11 (MAP3K) assembles with the other pathway components Ste7 (Sterile7; MAP2K), and the MAPK Fus3 at the membrane by binding to the scaffold protein Ste5 (Choi et al., 1994; Marcus et al., 1994; Printen \& Sprague, 1994; Kranz et al., 1994; Pryciak \& Huntress, 1998), and the adaptor protein Ste50 (Sterile50; Wu et al., 1999; Xu et al., 1996). The MAPK Fus3 as well as the partially redundant MAPK Kss1 are phosphorylated in response to pheromones by Ste7. However, only Fus3 interacts with the scaffold protein Ste5 (Bardwell et al., 1998; Sabbagh et al., 2001; Good et al., 2009). Phosphorylated Fus3 migrates to the nucleus and activates the transcription factor Ste12 (Sterile12), which forms homodimers and regulates mating-specific pheromone responsive genes (Van Drogen et al., 2001). Besides, Fus3 targets the transcription factor Tec1 (Transposon enhancement control) for degradation by phosphorylation (Chou et al., 2006).

The invasive filamentous growth MAPK pathway is activated upon nutrient limitation by the transmembrane receptors Sho1 (Synthetic high osmolarity-sensitive) and Msb2 (Multicopy suppression of a budding defect; Mösch et al., 1999; Hamel et al., 2012; Figure $3 \mathrm{~A}$ ). Heterodimers of Ste12 and Tec1 can be formed and activate filamentation specific genes (Chou et al., 2006).

The invasive filamentous growth and the pheromone response MAPK pathways share several components, including the core MAPK module consisting of Ste11, Ste7, and Kss1, as well as the adaptor protein Ste50, and the kinase Ste20, but activate different responses. The yeast MAPK Kss1 phosphorylates the transcription factors Ste12, which interacts with Tec1 upon nutrient-rich conditions and induces invasive or pseudohyphal growth (Hamel et al., 2012). 
A

Saccharomyces cerevisiae

B Aspergillus nidulans Verticillium dahliae

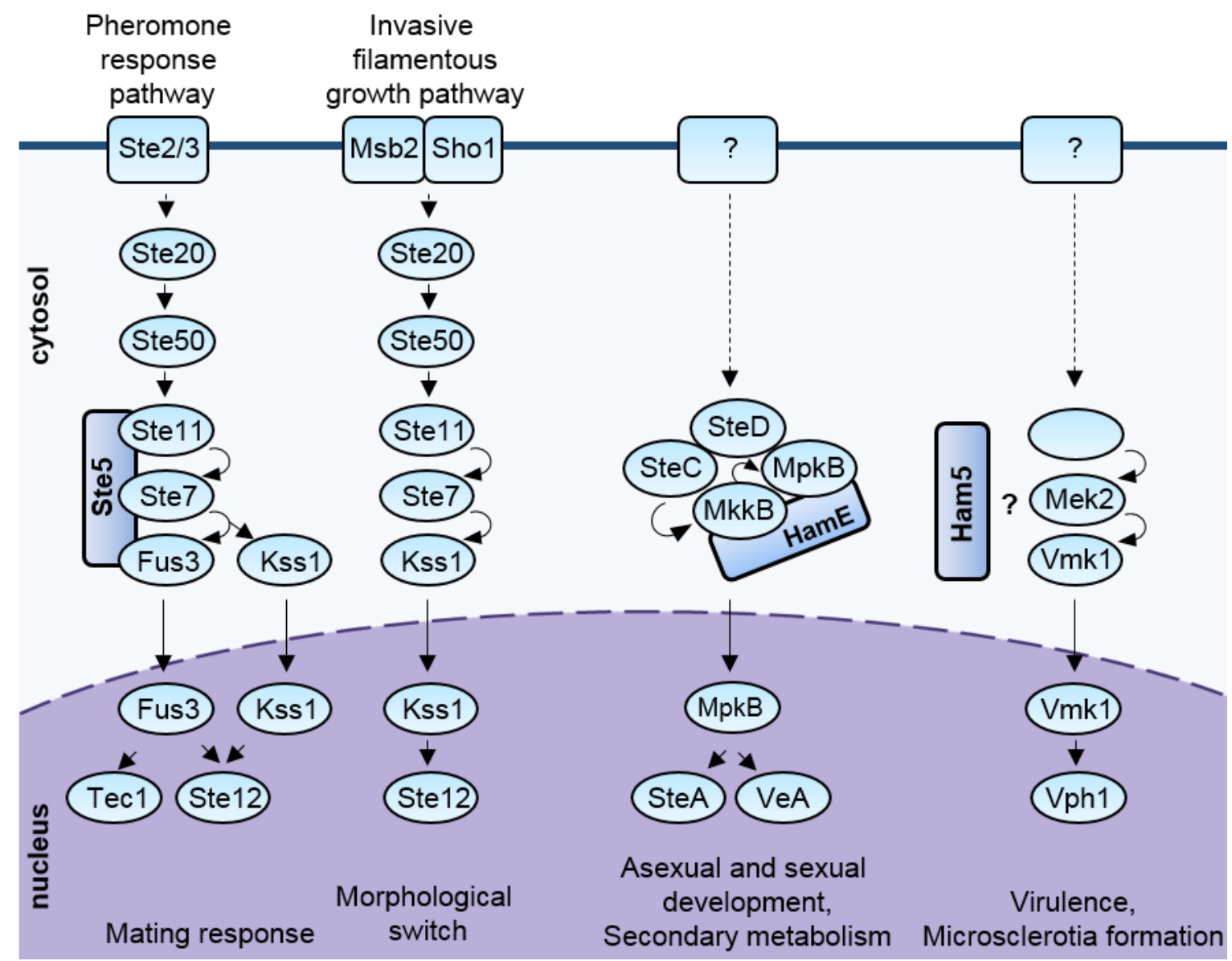

Figure 3: The yeast Fus3 and Kss1 MAPK pathways and homologous cascades in filamentous ascomycetes $\boldsymbol{A}$. nidulans and V. dahliae. (A) In S. cerevisiae the scaffold protein Ste5 is required for assembly and maintenance of pathway specificity in response to pheromones. This leads to phosphorylation of the transcription factors Ste12 and Tec1. Ste12 regulates mating responsive genes. Phosphorylated Tec1 is targeted for degradation. The invasive filamentous growth pathway shares the core MAPK module, but is independent from Ste5. It induces a morphological switch in response to starvation, which is regulated by Tec1-Ste12 heterodimers (modified from Hamel et al., 2012). (B) In A. nidulans the Ste5 orthologous scaffold protein HamE interacts with the MAP2K MkkB and the MAPK MpkB. The MAP3K SteC interacts with the Ste50-like adaptor protein SteD and the MAP2K MkkB. The cascade results in phosphorylation of the Ste12-like transcription factor SteA and the velvet protein $\mathrm{VeA}$ in the nucleus and, thereby, regulates genes involved in asexual and sexual development and secondary metabolism (based on Frawley et al., 2018). (C) In plant pathogenic fungi the Fus3/Kss1-like MAPK is essential for pathogenicity, as Vmk1 in $V$. dahliae. Requirement of a scaffold protein in this pathway was not described in any pathogenic filamentous ascomycete, whereas several components of the cascade were described in many organisms. In V. dahliae only the MAPK Vmk1 and the Ste12-like transcription factor Vph1 were characterized (Sarmiento-Villamil et al., 2018; Rauyaree et al., 2005). The other components of the MAPK core module in $V$. dahliae are not yet described. During this study the MAP2K Mek2 and the A. nidulans HamE/ N. crassa HAM-5 homolog in $V$. dahliae were characterized. (More details are given in the text). 


\subsubsection{Scaffolds in Fus3/Kss1 MAPK signaling in filamentous ascomycetes}

Whereas many components of the yeast Fus3/Kss1 MAPK homologs were studied in filamentous ascomycetes for years, orthologs to the scaffold protein Ste5 were identified only recently.

The first ortholog to the yeast scaffold Ste5 encoding gene was identified in the ascomycete Neurospora crassa (Dettmann et al., 2014; Jonkers et al., 2014). During chemotropic growth, the $N$. crassa scaffold protein HAM-5 (Hyphal anastomosis mutant 5) assembles a MAPK cascade of NRC-1 (MAP3K), MEK-2 (MAP2K) and MAK-2 (MAPK), anchored to the membrane via the adaptor protein STE-50 (Dettmann et al., 2014; Jonkers et al., 2014). The scaffold protein HAM-5 directly interacts with STE-50 and the MAPK MAK-2, whereas indirect interaction was proposed with the MAP2K MEK-2 and the MAP3K NRC-1 (Dettmann et al., 2014). HAM-5 was described to be essential for oscillation of the MAPK module assembly and disassembly at opposing tips of germlings or hyphae leading to fusion in N. crassa (Fleissner et al., 2009; Aldabbous et al., 2010; Fu et al., 2011; Dettmann et al., 2014; Jonkers et al., 2014).

A homolog to N. crassa HAM-5, named HamE, was identified in Aspergillus nidulans (Frawley et al., 2018). HamE was proposed as a scaffold of the pheromone pathway with roles in regulation of sexual and asexual development as well as in secondary metabolite production (Bayram et al., 2012; Frawley et al., 2018). HamE interacts with the MAP2K MkkB and the MAPK MpkB, but not with the MAP3K SteC (Bayram et al., 2012; Frawley et al., 2018). The MAPK module is anchored to the membrane via the yeast Ste50 homolog SteD (Bayram et al., 2012; Frawley et al., 2018; Figure 3B). In A. nidulans, HamE is required for efficient phosphorylation of the MAPK, which regulates the yeast Ste12-like transcription factor SteA as well as the velvet protein VeA, both important for fungal development and secondary metabolite production (Bayram et al., 2012; Frawley et al., 2018).

\subsubsection{Fus3/Kss1 MAPK signaling in plant pathogenic fungi}

MAPK cascades of both partners are involved in the establishment of the plant-pathogen interaction (Hamel et al., 2012). In response to the recognition of pathogen-associated molecular patterns (PAMPs) the plant activates defense genes via MAPK cascades and calcium signaling, known as PAMP-triggered immunity (Ali \& Reddy, 2008). In ascomycetous plant pathogens MAPK pathways corresponding to the $S$. cerevisiae Fus3/Kss1, Slt2, and Hog1 cascades can be found (Turrà et al., 2014). The MAPK pathways display conserved, as well as species-specific roles, as they are involved in chemotropic sensing of plant root exudates, regulation of adhesion, infection-related morphogenesis, cell wall composition, stress response, and formation of dormant 
structures (Xu, 2000; Zhao et al., 2007; Ali \& Reddy, 2008; Rispail et al., 2009; Rana et al., 2017).

The best described MAPK in phytopathogens with the highest conservation in its impact on virulence is the Fus3/Kss1 MAPK. This pathway controls virulence in diverse fungal pathogenic species with different life styles, developing different infection structures, and colonizing plants from different infection sites, including stomata, roots, and plant surfaces (Hamel et al., 2012). In appressoria-forming plant pathogenic ascomycetes as Magnaporthe oryzae, the Fus3/Kss1-like MAPK is essential for appressorium formation (Xu \& Hamer, 1996; Lev et al., 1999; Takano et al., 2000; Ruiz-Roldán et al., 2001; Hamel et al., 2012; Jiang et al., 2018a). MAPK-deficient mutants of the nonappressorium forming multi-host pathogen F. oxysporum displayed defects in plant penetration as well, correlated with decreased gene expression of pectate lyases (Di Pietro et al., 2001). In contrast, the MAPK is dispensable for pathogenicity in mice (Ortoneda et al., 2004). A role of the Fus3/Kss1-like MAPK in regulation of cell wall degrading enzymes (CWDEs) was observed as well in other phytopathogens (Lev \& Horwitz, 2003; Jiang et al., 2018a). The pathway displays positive regulatory effects on secondary metabolite and sclerotia production in some species (Chen et al., 2004; Jiang et al., 2018a). Upstream components of the cascade, like the MAP2K Ste7 and the MAP3K Ste11, as well as the adaptor protein Ste50, and homologs of the downstream transcription factor Ste12 display similar roles in virulence of different plant pathogenic fungi (Park et al., 2002, 2006; Zhao et al., 2005; Zhao \& Xu, 2007; Rispail \& Di Pietro, 2009; Li et al., 2012).

In V. dahliae only the MAPK homolog Vmk1 and the downstream Ste12-like transcription factor Vph1 were characterized (Figure 3C). A conserved essential role in pathogenicity was described for Vmk1 and MAPK-deficient mutants displayed reduced conidiation and microsclerotia formation (Rauyaree et al., 2005). No alterations in their ex planta phenotype or in conidiation, but impaired hyphopodia formation and penetration of the root cortex were observed for Vph1-deficient mutants (Sarmiento-Villamil et al., 2018).

A scaffold protein of the Fus3/Kss1-like MAPK signaling pathway was not yet analyzed in any filamentous phytopathogen. However, homologs to N. crassa HAM-5 were described to be highly conserved among Pezizomycotina, the biggest subdivision of the Ascomycota, before its scaffold-function was described in any species (Jamet-Vierny et al., 2007). In this work, the role of the HAM-5 homolog was studied in V. dahliae combined with the characterization of the MAP2K homolog Mek2, and the MAPK Vmk1 as control. 


\subsection{The unfolded protein response}

Perception of the plant host is transduced by intracellular fungal transduction pathways leading to the upregulation of several genes encoding for proteins involved in plant host colonization. Many of these proteins have to be secreted to facilitate their role in hostfungus interactions. In hemibiotrophic fungi, secretion occurs in waves to induce an initial biotrophic interaction followed by a necrotrophic phase. During initial invasion of the host barriers, extracellular degradation enzymes like CWDEs, or hydrolytic enzymes are required. Subsequent systemic colonization relies on successful adaptation to a new environment by establishment of the nutrient uptake and suppression of the host immune system by effectors or toxic proteins. In later stages, lytic enzymes and defense response activating effectors are secreted (O'Connell et al., 2012; Lo Presti et al., 2015). These processes require increased protein processing and preparation for secretion via the Golgi apparatus, which is accomplished by the endoplasmic reticulum (ER).

The ER is a branched membrane system fused with the outer membrane of the nucleus, which is able to adapt to developmental or environmental conditions (Schwarz \& Blower, 2016). In situations requiring increased protein folding and secretion capabilities, the ER senses the imbalance of incoming proteins and protein folding capacity of the cell organelle and triggers expression of genes involved in ER stress relief, referred to as unfolded protein response (UPR) (Kozutsumi et al., 1988; Kohno et al., 1993; Hetz, 2012; Heimel, 2015). These genes encode chaperones, foldases, glycosylation enzymes, as well as proteins required for vesicle transport, ER-associated degradation (ERAD), lipid biosynthesis, and regulators for adaptation of the ER size (Cox et al., 1993; Kaufman, 1999; Travers et al., 2000; Conn, 2011; Hetz, 2012). Whereas in mammals three ER transmembrane receptors, IRE1, PERK, and ATF6 are involved in UPR signaling to the nucleus (Ron \& Walter, 2007; Hetz, 2012), in fungi, only the signal transduction pathway initiated by the sensor Ire1 (Inositol requiring 1) was described (Mori et al., 1993; Okamura et al., 2000; Kohno, 2010). This pathway is conserved in eukaryotes, however, species-specific adaptations can be observed. Especially the function of the basic leucine zipper (bZIP) transcription factor Hac1 (Homologous to Atf/Creb1) varies between different organisms and will be focused during this work in respect to its role in fungal differentiation and virulence of $V$. dahliae. In the following, the UPR pathway of $S$. cerevisiae will be summarized as paradigm and UPR functions in pathogenic fungi will be introduced.

\subsubsection{The unfolded protein response pathway in Saccharomyces cerevisiae}

ER stress causes a developmental block in the transition from the yeast form to pseudohyphal growth and sporulation in S. cerevisiae (Schröder et al., 2000). The yeast 
unfolded protein response is based on perception of un- or misfolded proteins in the ER lumen by the transmembrane sensor Ire1 (Mori et al., 1993; Cox et al., 1993; Figure 4). Ire1 possesses a three domain structure: an ER luminal $\mathrm{N}$-terminal domain, as well as the cytoplasmic kinase, and the endoribonuclease domains. In presence of un- or misfolded proteins, Ire1 oligomerizes and proteins auto-phosphorylate each other via the cytoplasmic kinase domains and the cytoplasmic endoribonuclease domain is activated (Shamu \& Walter, 1996; Welihinda \& Kaufman, 1996; Sidrauski \& Walter, 1997; Okamura et al., 2000; Figure 4).

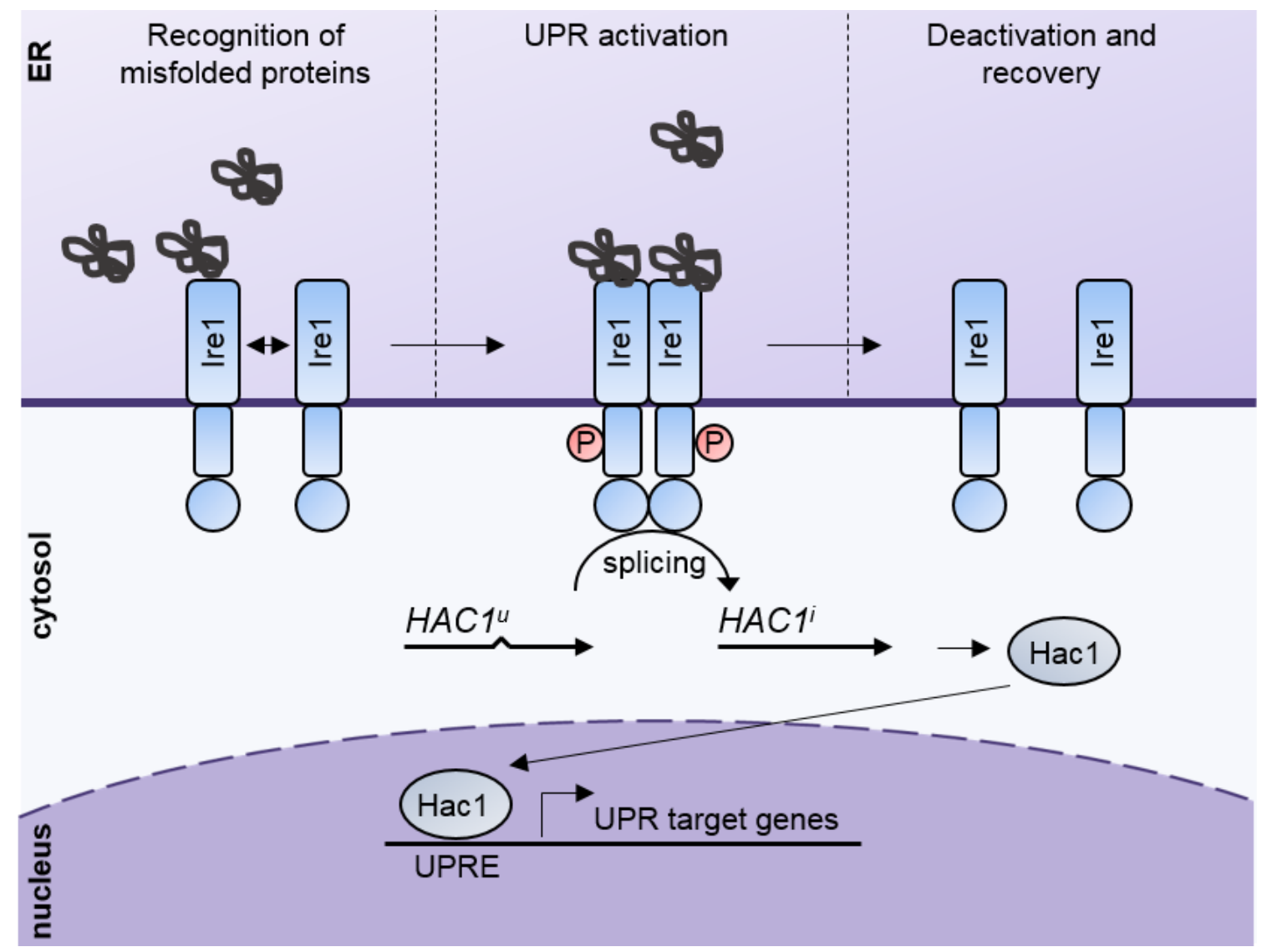

Figure 4: Hac1 is the central unfolded protein response regulator in S. cerevisiae. ER stress is perceived by the transmembrane sensor Ire1 in the presence of un- or misfolded proteins in the ER lumen. Ire1 proteins oligomerize and activate autophosphorylation by its cytosolic kinase domain. Thereby, the endoribonuclease domain of Ire1 is activated, resulting in unconventional splicing of the HAC1 ${ }^{u}$ mRNA. The spliced HAC1 mRNA (HAC1) is translated into the bZIP transcription factor Hac1. Hac1 migrates to the nucleus and regulates UPR target genes with unfolded protein response elements (UPRE) in their promotor regions, like genes encoding for chaperones, foldases, or genes involved in ERassociated degradation (ERAD) or ER expansion, resulting in ER stress relief (based on Heimel, 2015). 
The endoribonuclease activity is required for unconventional splicing of the bZIP transcription factor Hac1 encoding mRNA (Sidrauski \& Walter, 1997; Gonzalez et al., 1999; Figure 4). Subsequent ligation of the exons is processed by the tRNA ligase Trl1 (Sidrauski et al., 1996; Sidrauski \& Walter, 1997). Translation of the unspliced mRNA variant into $\mathrm{Hac}^{4}{ }^{\mathrm{u}}$ is suppressed by base-pairing interaction between the unconventional intron and the 5'UTR (Chapman \& Walter, 1997; Rüegsegger et al., 2001).

Furthermore, translation of the unspliced mRNA correlated with accelerated degradation of very unstable Hac1u proteins was suggested (Di Santo et al., 2016). Splicing of the $H A C 1^{u}$ mRNA results in the mRNA variant $H A C 1^{i}$, which is translated into the stable Hac1 protein. Hac1 migrates into the nucleus where it regulates UPR target genes (Mori et al., 1996, 1998; Figure 4). Several of these genes possess a specific palindromic sequence, named UPR element (UPRE), in their promotor regions (Mori et al., 1996, 1998). Expression of UPR target genes mediates ER stress relief by increasing the folding capacity, ER expansion and degradation of misfolded proteins via the ERAD pathway (Cox et al., 1993; Kaufman, 1999; Travers et al., 2000; Jonikas et al., 2009; Heimel, 2015).

\subsubsection{The unfolded protein response pathway in pathogenic fungi}

Ire1-dependent UPR signaling for regulation of genes involved in ER stress relief was observed as a conserved mechanism for ER stress relief and virulence of several pathogenic fungal species colonizing animal or plant hosts (Cheon et al., 2011; Joubert et al., 2011; Richie et al., 2011; Krishnan \& Askew, 2014; Heimel, 2015). The role of homologs and orthologs of the UPR regulatory transcription factor Hac1 varies in human or plant pathogenic fungal species (Krishnan \& Askew, 2014).

The ER stress response mechanism of the opportunistic human pathogenic yeast Candida glabrata is regulated in an Ire1-dependent decay independently of Hac1 (Miyazaki et al., 2013). This results in splicing of various ER-associated mRNAs by Ire1 as primary mechanism to cope with ER stress (Miyazaki et al., 2013; Heimel, 2015).

In yeast and filamentous ascomycetes, translation of the uninduced HAC1 mRNA variant into an alternative protein $\mathrm{Hac}{ }^{4}$ was described to be blocked by different mechanisms (Chapman \& Walter, 1997; Rüegsegger et al., 2001; Saloheimo et al., 2003; Mulder et al., 2004; Joubert et al., 2011; Heimel, 2015). In the basidiomycete U. maydis, translation of the unspliced cib1 (Clp1 interacting bZIP1) mRNA into Cib1 ${ }^{\text {u}}$, possessing a UPR repressing function and an additional Cib1-independent function in ER stress response, was assumed similar to the mechanisms observed in higher eukaryotes (Yoshida et al., 2006, 2009; Heimel et al., 2013). 
Hac1 homologs and orthologs are required for regulation of vegetative growth under ER stress inducing growth conditions in most tested fungal species (Richie et al., 2009; Cheon et al., 2011; Joubert et al., 2011; Heimel et al., 2013; Montenegro-Montero et al., 2015). However, the impact of the UPR on vegetative growth under non-stress conditions is species-specific and varies from unaltered growth in absence of ER stressors (Cheon et al., 2011; Heimel et al., 2013; Heimel, 2015), over impacts on conidia or cell morphology (Wimalasena et al., 2008; Joubert et al., 2011), to functions specifically important on complex substrates (Richie et al., 2009; Montenegro-Montero et al., 2015).

A crosstalk between the cell wall integrity (CWI) MAPK pathway and the UPR pathway was suggested following the observation that UPR-deficient strains displayed increased sensitivity not only in response to ER stressors, but as well to cell wall perturbing agents, as for example in C. albicans, C. neoformans, and A. fumigatus (Richie et al., 2009, 2011; Cheon et al., 2011; Malavazi et al., 2014). In the necrotrophic plant pathogenic fungus $A$. brassicicola, the loss of virulence of mutants deficient in the UPR pathway was suggested to be caused by increased susceptibility to antimicrobial plant metabolites inducing membrane damage (Joubert et al., 2011; Guillemette et al., 2014). In contrast, sensitivity to cell wall perturbing agents was unaffected in Hac1-deficient strains of the saprophytic fungus $N$. crassa (Montenegro-Montero et al., 2015).

Besides the role in counteracting host antimicrobial compounds in A. brassicicola, the UPR pathway was described to be linked to infection-related morphogenesis, adaptation of the secretion capacity during plant invasion and colonization, and recently also to the regulation of ER stress independent virulence factors (Cheon et al., 2011; Joubert et al., 2011; Richie et al., 2011; Heimel et al., 2013; Hampel et al., 2016; Pinter et al., 2019). In the rice blast fungus $M$. oryzae, homologs of the heat shock protein Bip1 and the bZIP transcription factor Hac1 are involved in induction of the ER stress response and are essential for asexual development and penetration of the plant surface (Yi et al., 2009; Tang et al., 2015; Jiang et al., 2018b). Appressorium formation and initial penetration of the plant surface was unaffected in the avirulent $A$. brassicicola mutant defective in the UPR regulator AbHacA (Joubert et al., 2011). In the dimorphic corn smut fungus U. maydis, a morphogenic switch from budding to filamentous growth initiates pathogenic development (Boyce et al., 2005). Here, a functional UPR is required for the mitotic growth of the fungus within the cell after formation of appressoria and penetration of the plant surface (Heimel et al., 2010). Regulation of virulence-specific genes, like the effector genes pit2 and tin1-1, were identified to be regulated by the UPR in the basidiomycete (Hampel et al., 2016). Recently, a UPR-regulated virulence factor, the signal peptide peptidase Ssp1 with specific function in interference with plant defense 
responses and dispensable for ER stress resistance, was identified in $U$. maydis (Pinter et al., 2019).

Overall, the UPR pathway plays important roles in developmental processes and plant infections in several fungi. None of the UPR components was studied in Verticillia to date. During this study, the homolog of the bZIP transcription factor Hac1 was characterized in $V$. dahliae.

\subsection{Oxylipins}

The fungal secretome does not only include proteins, but also lipid metabolites, as oxygenated polyunsaturated fatty acids, named oxylipins. These fungal hormones act as trans-kingdom signaling molecules as they show conserved structures in fungi and plants (Brodhun \& Feussner, 2011). Fungal oxylipins and their polyunsaturated fatty acid precursors are involved in fungal development and secondary metabolite production as well as in environmental adaptation. The signaling molecules were described to manipulate plant cellular processes in favor of the survival of the invader within the plant and, on the other hand, plant oxylipins can modulate fungal development (Calvo et al., 2001; Brodhagen et al., 2008; Brodhun et al., 2009; Reverberi et al., 2010; Scala et al., 2014). Oxylipins contribute to fungal differentiation and might affect the outcome of the interaction of Verticillia with their hosts, which was analyzed in frame of this study. In the following, the role of fungal oxylipins in development and plant-fungus interactions will be introduced.

\subsubsection{Oxylipins in fungal development}

In fungi, fatty acid synthases produce long chain fatty acids. Unsaturated and polyunsaturated fatty acids are produced by introduction of double bonds by ER membrane-bound desaturases. These desaturases transfer electrons from the donor cytochrome $b_{5}$ and thereby reduce molecular oxygen to two molecules of water (Uttaro, 2006). The polyunsaturated fatty acid linoleic acid $(18: 2 \Delta 9,12)$ is synthesized by oleate $\Delta 12$-fatty acid desaturases from oleic acid (18:1 $\Delta 9)$ by introduction of a second double bond into the carbon chain at position 12 from the carboxy-terminus (Uttaro, 2006; Figure 5). Linoleic acid is the most abundant fatty acid in mycelia of several fungal species and displays an important membrane component with functions in adaptation of membrane fluidity and the major precursor of fungal oxylipins (Rambo \& Bean, 1974; Evans et al., 1986; Castoria et al., 1995; Goodrich-Tanrikulu et al., 1998; Los \& Murata, 1998; Gostinčar et al., 2009; Brodhun \& Feussner, 2011). 


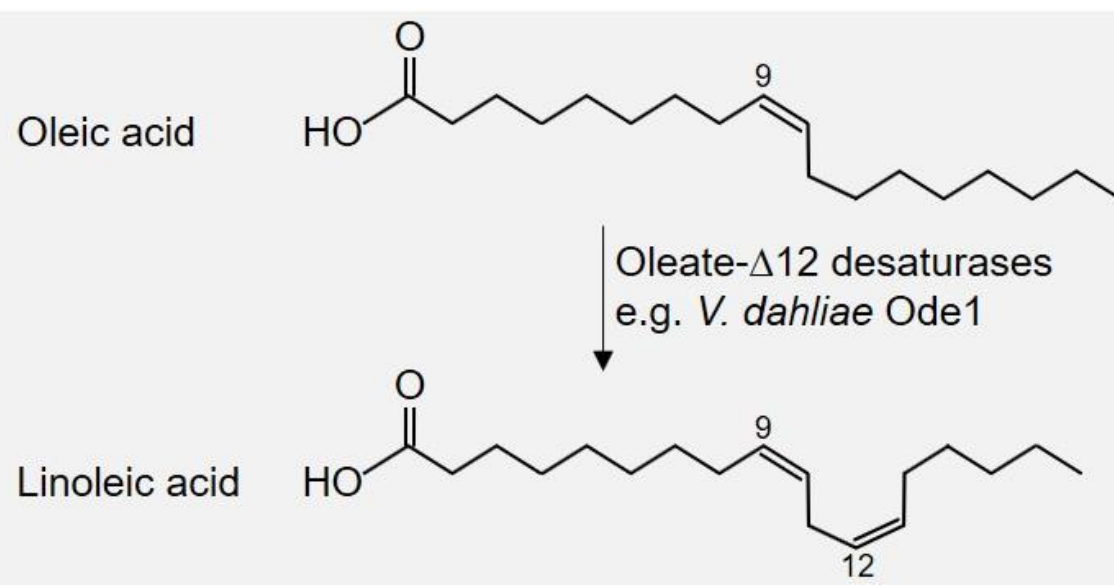

Figure 5: Linoleic acid biosynthesis by oleate $\Delta 12$-fatty acid desaturases. Linoleic acid is the most abundant fatty acid in fungal cells as an important component of the cell membrane and the major precursor of oxylipins (Rambo \& Bean, 1974; Evans et al., 1986; Castoria et al., 1995; Goodrich-Tanrikulu et al., 1998; Los \& Murata, 1998; Gostinčar et al., 2009; Brodhun \& Feussner, 2011). Linoleic acid is synthesized by oleate $\Delta 12$-fatty acid desaturases, like OdeA in Aspergilli or OdeA corresponding protein Ode1 in $V$. dahliae, from oleic acid by introduction of a second double bond into the carbon chain at position 12 from the carboxy-terminus (Calvo et al., 2001; Chang et al., 2004; Wilson et al., 2004; Uttaro, 2006).

In plants, lipoxygenases (LOXs) catalyze the initial step in oxylipin biosynthesis from polyunsaturated fatty acids whereas different enzymes catalyze oxylipin producing reactions from polyunsaturated fatty acid substrates in fungi (Brodhun \& Feussner, 2011). These enzymes group into monooxygenases, LOXs, and cyclooxygenases (Fischer \& Keller, 2016). The cyclooxygenases include the linoleate diol synthases (LDSs), also known as precious sexual inducer (Psi) factor-producing oxygenase (Ppo) enzymes (Andreou \& Feussner, 2009; Brodhun \& Feussner, 2011). Oxylipins were found to play significant roles in the coordination of developmental processes like conidiation, sclerotia formation, production of secondary metabolites, and quorum sensing (Calvo et al., 2001; Brodhun et al., 2009; Reverberi et al., 2010; Brodhun \& Feussner, 2011; Scala et al., 2014).

In A. nidulans and Aspergillus parasiticus, mutants deficient in the conversion of oleic acid into linoleic acid as the major precursor of oxylipins were analyzed by the deletion of the oleate $\Delta 12$-fatty acid desaturases OdeA (Calvo et al., 2001; Chang et al., 2004; Wilson et al., 2004). Mutants of both species displayed reduced vegetative growth and conidiation as well as altered ascospore formation. Additionally, a complete loss of sclerotia development was observed in the A. parasiticus odeA-deficient mutant (Chang et al., 2004; Wilson et al., 2004).

A. nidulans produces Psi factors, which promote sexual development (Champe \& ElZayat, 1989). Aspergilli possess three to four Ppo enzymes (PpoA, PpoB, PpoC, PpoD) 
producing a ratio of these hormones to regulate the balance of asexual to sexual spores (Mazur \& El-zayat, 1991; Tsitsigiannis et al., 2004b, 2004a, 2005a, 2005b; Tsitsigiannis \& Keller, 2007; Horowitz Brown et al., 2009). Whereas products from PpoA and PpoB induce sexual sporulation, products of $\mathrm{PpoC}$ induce asexual sporulation in A. nidulans (Champe \& El-Zayat, 1989; Tsitsigiannis et al., 2004a, 2004b). Ppo enzymes also affect the balance between asexual and sexual spores in $A$. fumigatus, however, the effect of the different enzymes varies (Dagenais et al., 2008). Aspergillus flavus produces a fourth Ppo enzyme, PpoD (Horowitz Brown et al., 2009). In this organism, Ppo enzymes as well as a LOX, were found to regulate the density-dependent morphological transition of conidia to sclerotia as resting structures (Horowitz Brown et al., 2008, 2009). A negative regulatory impact of a LOX on sclerotia production has been observed in Aspergillus ochraceus (Reverberi et al., 2010). Besides their roles in regulation of sexual development and quorum sensing, Ppo enzymes are involved in secondary metabolite production in Aspergilli as they promote, for instance, the biosynthesis of the mycotoxins sterigmatocystin as well as aflatoxin and inhibit the production of penicillin (Tsitsigiannis \& Keller, 2006; Horowitz Brown et al., 2009). Homologous ppo genes were identified in several ascomycete and basidiomycete fungal species (Tsitsigiannis et al., 2005b; Tsitsigiannis \& Keller, 2007).

In summary, linoleic acid and oxylipin producing enzymes play crucial roles in fungal growth, development, and secondary metabolite production, which are species-specific and, in some cases, display antagonistic effects.

\subsubsection{Oxylipins in plant-fungus interactions}

Plant oxylipins were found to modulate fungal development and mycotoxin production and, vice versa, fungal oxylipins can manipulate the host lipid metabolism and alter plant defense responses presumably by mimicking endogenous signal molecules. In several cases, the interplay of plant and fungal oxylipins decides on the outcome of the interaction.

The plant stress and defense response to pathogens with different lifestyles is mediated by the hormone jasmonic acid (JA) and JA derivatives synthesized from linoleic acid (Thaler et al., 2004) (Figure 6). Plants deficient in JA biosynthesis display increased susceptibility, for instance to Verticillia and F. oxysporum (Thaler et al., 2004; Thatcher et al., 2009; Riemann et al., 2013; Fischer \& Keller, 2016; Scholz et al., 2018). Fungal species were described to produce oxylipins similar to the plant hormone JA and active or inactive JA derivatives to manipulate host defense reactions as, for example, F. oxysporum and M. oryzae (Husain et al., 1993; Miersch et al., 1999; Christensen \& Kolomiets, 2011; Brodhun \& Feussner, 2011; Andolfi et al., 2014; Cole et al., 2014; 
Patkar \& Naqvi, 2017; Chini et al., 2018). Due to structural similarities, fungal oxylipins were suggested to be perceived by plant receptors and to mimic plant hormones for manipulation of the immune response (Feys, 1994; Brodhun \& Feussner, 2011). The only known JA receptor to date is the F-box protein jasmonate receptor coronatine insensitive 1 (COI1) (Brodhun \& Feussner, 2011). COl1 acts as a de-repressor of JA responsive genes by targeting the JAZ (jasmonate ZIM-domain) repressor proteins for degradation (Thines et al., 2007). COl1-deficient $A$. thaliana mutants are insensitive to perception of activated jasmonoyl isoleucine (JA-lle) derivatives. F. oxysporum and $V$. longisporum were found to induce disease symptoms in Arabidopsis plants dependent on the presence of COI1 (Thatcher et al., 2009; Ralhan et al., 2012; Cole et al., 2014). However, this is restricted to isolates, which are able to produce JA derivatives in F. oxysporum (Cole et al., 2014). Tomato infecting F. oxysporum isolates do not produce detectable jasmonates and COI1 is dispensable for virulence on tomato plants (Cole et al., 2014).

Maize plants deficient in LOX3 displayed increased resistance to Fusarium verticilloides infection associated with decreased mycotoxin production (Gao et al., 2007). F. verticillioides susceptible varieties were found to produce high levels of LOX3 products and the precursor linoleic acid (Dall'Asta et al., 2012, 2015; Battilani et al., 2018). In F. verticillioides the Linoleate Diol Synthase 1 (LDS1)-derived oxylipins act as negative regulators of vegetative growth, conidiation, and secondary metabolite production (Scala et al., 2014). The signaling molecules induce LOX3, thereby, suppress the JA-mediated plant defense response and promote fungal virulence (Battilani et al., 2018). Here, oxylipins from both interaction partners are essential for susceptibility (Battilani et al., 2018).

In contrast to fungal oxylipins, which are mimicking plant hormones to suppress the plants defense responses, there are also examples for fungal oxylipins which activate plant defense responses for their benefit. The grapevine pathogen Lasiodiplodia mediterranea produces a JA precursor named JA ester lasiojasmonate A (LasA), which activates JA-regulated defense responses in plants presumably after conversion into the active JA-lle derivative (Chini et al., 2018). Fungal LasA was suggested to be produced in late stages of infection to induce cell death and facilitate fungal infection, but the mode of action of LasA has not been elucidated to date (Chini et al., 2018).

Like pathogenic species, mutualistic fungi found their path to manipulate the host defense responses in order to establish their interaction (Patkar \& Naqvi, 2017; Sanders, 2011). Manipulation of the host via oxylipins from mutualistic fungi was not described to date, whereas there are examples of fungal effectors targeting the plant's jasmonate signaling pathway from Trichoderma virens and mycorrhizal fungi as Laccaria bicolor 
(Djonovic et al., 2007; López-Ráez et al., 2010; Christensen \& Kolomiets, 2011; Plett et al., 2014; Patkar \& Naqvi, 2017).

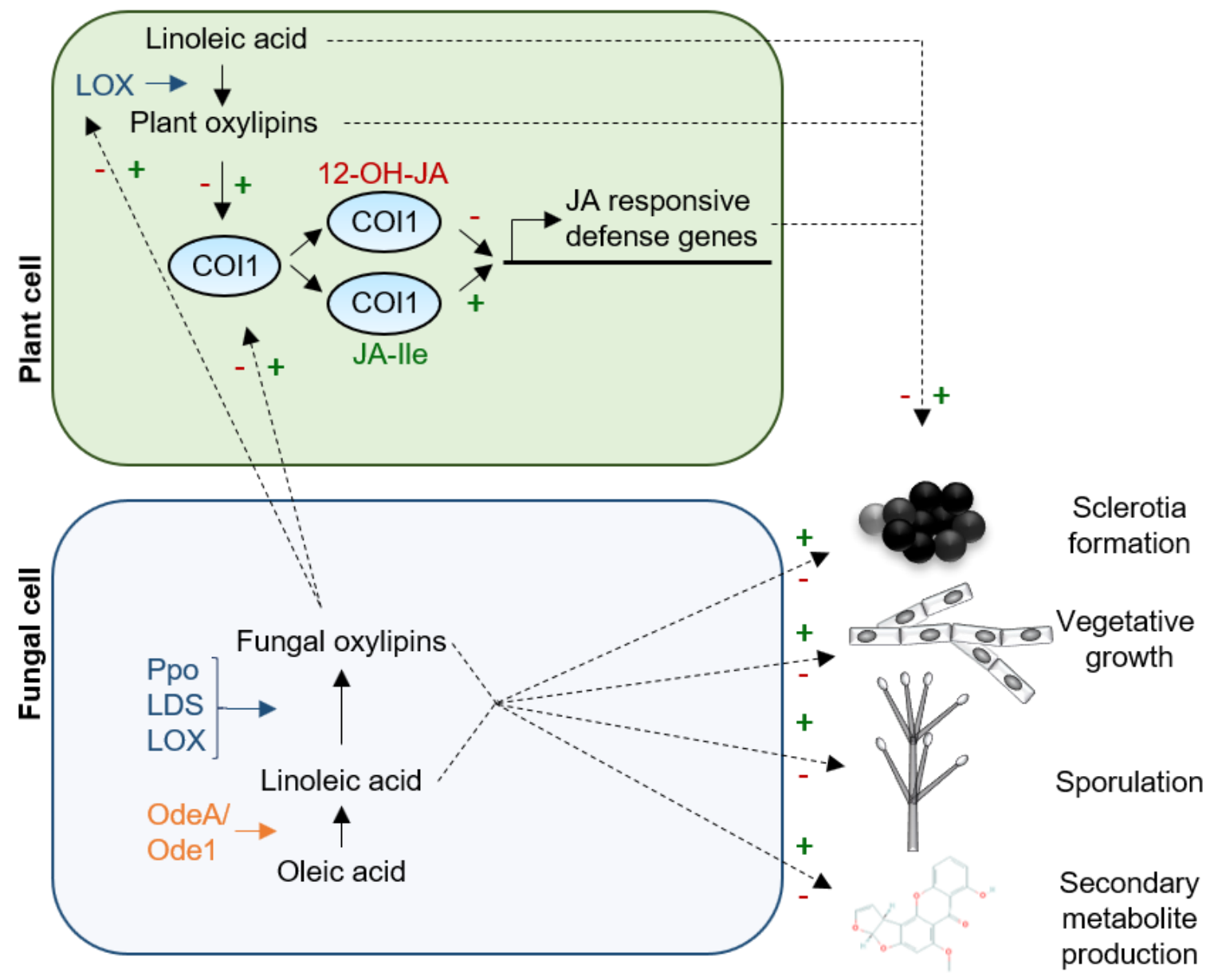

Figure 6: Fungal and plant oxylipins in crosstalk and fungal differentiation. Fungi synthesize oxylipins predominantly from linoleic acid, a polyunsaturated fatty acid which is produced by oleate $\Delta 12$-fatty acid desaturases like OdeA in Aspergilli (Calvo et al., 2001; Chang et al., 2004; Wilson et al., 2004) or the corresponding V. dahliae desaturase Ode1. The conversion of linoleic acid into fungal oxylipins is catalyzed by different enzymes including precious sexual inducer (Psi) factor-producing oxygenase (Ppo) enzymes, linoleate diol synthases (LDS), and lipoxygenases (LOX) (Andreou \& Feussner, 2009; Brodhun \& Feussner, 2011; Patkar et al., 2015; Fischer \& Keller, 2016). Fungal oxylipins display positive and negative regulatory effects (indicated by arrows with plus and minus) on fungal growth, conidiation, sclerotia formation, and mycotoxin production. Furthermore, fungal oxylipins manipulate the host's defense responses either by targeting the plant's biosynthesis of oxylipins or the jasmonate (JA) binding F-box protein coronatine insensitive 1 (COI1) (indicated by arrows with plus and minus). Binding of active JA derivatives like jasmonoyl isoleucine (JA-lle) to COl1 targets the jasmonate ZIM-domain (JAZ) repressors of JA responsive genes to the degradation machinery and, thereby, activates defense responses. Binding of 12-hydroxy JA (12-OH-JA) inactivates $\mathrm{COI} 1$ and negatively regulates JA responsive defense genes. Plant oxylipins regulate their defense responses and can modulate fungal growth, differentiation, and mycotoxin production.

In summary, the communication of fungi and plants via the production of endogenous and perception of exogenous oxylipins determines the susceptibility of the host plant or 
the propagation of the fungus (Figure 6). To date, these enzymes were not analyzed in Verticillia and their role in fungal growth, development, and virulence is unknown. In this work $V$. dahliae ODE1 coding for the oleate $\triangle 12$-fatty acid desaturase was identified for further characterization of its role in differentiation and virulence (Figure 5, Figure 6).

\subsection{Aim of the study}

Plant colonizing fungi and their hosts coevolve and develop constantly new specialized tools like effectors, oxylipins, mycotoxins, and CWDEs to benefit from or overcome the interaction. To succeed in colonization, these tools have to be prudently orchestrated during the different steps in colonization, which requires perception and adaptation to environmental conditions regulated by endogenous signaling cascades. Conserved signaling pathways were shown to be modified in several species in frame of niche adaptation.

The independently evolved $V$. longisporum lineages adapted to Brassicaceae as a host but display differences in their pathogenicity. In the course of this study, two $V$. longisporum isolates from different lineages and pathotypes but from fields in the same area in Germany (Zeise \& von Tiedemann, 2002) were compared to investigate the genomic basis for the establishment of different pathotypes in related species. In the genomes of $V$. dahliae, a relative enrichment of genes with functions in niche adaptation was observed in LS regions (de Jonge et al., 2013; Gibriel et al., 2019). To date, LS regions in the genome of the hybrid $V$. longisporum were not analyzed. In first experiments, the colonization behavior of the two $V$. longisporum isolates in rapeseed plants was compared. Subsequently, LS regions present in the pathogenic and absent in the mutualistic colonizer were searched. In order to narrow down to interesting regions, an LS region originating from the $V$. dahliae-related parental species was focused. Deletion strains of this region were constructed in the pathogenic $V$. longisporum isolate and the haploid relative $V$. dahliae to characterize the role of LS regions in adaptation of the rapeseed colonizer $V$. longisporum.

The pathotype of a fungus depends on both, the defense responses of the host plant and the orchestration of tools enabling plant colonization. Sensing of the fungal environment and signal transduction are important mechanisms for adaptation and colonization of plants. The Fus3/Kss1-like MAPK signaling pathway in $V$. dahliae has a conserved role in fungal pathogenicity and, furthermore, the MAPK Vmk1 was described to positively regulate differentiation processes (Rauyaree et al., 2005). Insulation of homologous pathways by a scaffold protein was recently elucidated in the filamentous ascomycetes N. crassa and A. nidulans (Dettmann et al., 2014; Jonkers et al., 2014; Frawley et al., 2018). Homologs to these scaffold proteins have been identified bioinformatically in the 
biggest subdivision of the Ascomycota, including Verticillia (Jamet-Vierny et al., 2007). However, the role of the homolog has not yet been studied in any pathogenic species. Here, the function of the $V$. dahliae homolog Ham5 on the Fus3/Kss1-like MAPK pathway-mediated regulation of fungal differentiation and virulence was analyzed. Mutants deficient in the pathway components Vmk1 (MAPK) and Mek2 (MAP2K) were compared to a Ham5-deficient strain and double deletion strains defective in either Ham5/Vmk1 or Ham5/Mek2 regarding their vegetative growth, their ability to form microsclerotia, and virulence on tomato plants.

Signaling from the membrane coordinates the expression of tools involved in host colonization. This correlates with increased demands for processing and secretion of proteins in the ER, which requires a functional unfolded protein response (UPR). Variations of the role of the transcription factor Hac1 in development and virulence in different fungal species were observed (Krishnan \& Askew, 2014). To date, the UPR is an unstudied field in Verticillia. In frame of this work, the role of the homologous bZIP transcription factor Hac1 in fungal differentiation, ER stress response, and virulence in $V$. dahliae was addressed. The HAC1 homolog was identified and the occurrence of alternatively spliced mRNA variants was verified. HAC1 deletion strains were constructed and complemented by ectopic integration of the alternative mRNA splice variants $H A C 1^{u}$ and $H A C 1^{i}$ with $3^{\prime}$-hemagluttinin tags or unspliced $H A C 1$. It was tested if translation of $\mathrm{HAC1}^{u}$ into a protein could be detected. The constructed strains were characterized regarding their vegetative growth under physiological and ER stress conditions, conidiation, formation of microsclerotia as resting structures, and virulence in tomato plants.

Besides protein tools, fungi and plants possess lipid metabolites as signaling molecules with significant impact on the outcome of the interaction (Brodhun \& Feussner, 2011; Christensen \& Kolomiets, 2011; Fischer \& Keller, 2016; Patkar \& Naqvi, 2017). Here, the so far unknown role of a functional oxylipin biosynthesis in growth, development, and virulence of $V$. dahliae was investigated. Therefore, an oleate $\Delta 12$-fatty acid desaturase, catalyzing the oxidation of oleic acid to the major oxylipin precursor linoleic acid, was identified. Localization of the enzyme was tested by fluorescence microscopy of GFP tagged versions. Mutants deficient in this desaturase were constructed and phenotypically analyzed in planta and ex planta. 


\section{Materials AND Methods}

Media, buffers, and solutions used in this study were prepared with chemicals provided by AppliChem GmbH (Darmstadt, Germany), BD Biosciences (Heidelberg, Germany), Biozym Scientific GmbH (Hessisch Oldendorf, Germany), Carl Roth GmbH\&Co.KG (Karlsruhe, Germany), Fluka (Neu-Ulm, Germany), Invitrogen (Karlsruhe, Germany), Merck KGaA (Darmstadt, Germany), Oxoid Deutschland GmbH (Wesel, Germany), Roche Diagnostics GmbH (Mannheim, Germany), Sigma-Aldrich Chemie GmbH (Munich, Germany), Serva Electrophoresis GmbH (Heidelberg, Germany), and VWR International $\mathrm{GmbH}$ (Darmstadt, Germany). The $\mathrm{pH}$ was determined with a WTW bench $\mathrm{pH} / \mathrm{mV}$ Routine meter pH 526 (Sigma-Aldrich, Munich, Germany).

Ampicillin (Carl Roth GmbH\&Co.KG), kanamycin (AppliChem), chloramphenicol (AppliChem), clonNAT nourseothricin dihydrogen sulfate (Werner Bioagents $\mathrm{GmbH}$, Jena, Germany), cefotaxime (Wako Chemicals GmbH, Neuss, Germany), and hygromycin B (InvivoGen, San Diego, CA, USA) were used for selection of microorganisms. Small-scale sterile filtration of solutions was performed with Filtropur filters with a pore size of $0.2 \mu \mathrm{m}$ (Sarstedt AG\&Co, Nuembrecht, Germany). Fungal spores were filtered using Miracloth (Calbiochem Merck, Darmstadt, Germany). The Coulter Particle Count and Size Analyzer Z2 and the appropriate Coulter Isoton II Diluent (both Beckman Coulter, Krefeld, Germany) were used for conidiospore counting.

Restriction enzymes were obtained from Thermo Fisher Scientific (Schwerte, Germany) and NEB (Frankfurt am Main, Germany). The GeneArt Seamless Cloning and Assembly Kit and CloneJET PCR Cloning Kit were used from Thermo Fisher Scientific. RNase A was provided by AppliChem. Phusion High-Fidelity DNA polymerase, Taq DNA polymerase, corresponding polymerase buffers, and deoxynucleotides were obtained from Thermo Fisher Scientific (Schwerte, Germany). Q5 High-Fidelity DNA polymerase was purchased from New England Biolabs (Ipswich, MA, USA). Primers were obtained from Eurofins Genomics $\mathrm{GmbH}$ (Ebersberg, Germany) and Sigma-Aldrich Chemie GmbH (Munich, Germany). PCRs were performed in T Professional Standard 96, T Professional Trio 48, and T Professional Standard 96 Gradient thermocyclers (Biometra $\mathrm{GmbH}$, Goettingen, Germany), and in Primus 96 Thermal Cyclers (MWG Biotech AG, Ebersberg, Germany). DNA was purified with the NucleoSpin Plasmid Kit or NucleoSpin Gel and PCR clean up Kit from Macherey-Nagel (Dueren, Germany). DNA and RNA concentrations were measured with a NanoDrop ND-1000 spectrophotometer from PeqLab Biotechnology GmbH (Erlangen, Germany). Protein concentrations were measured with an Infinite M200 microplate reader operated with Magellan software (both: Tecan Trading AG, Maennedorf, Switzerland). 
RNA was extracted with the Direct-zol RNA MiniPrep Kit (Zymo Research, Freiburg im Breisgau, Germany). The GeneRuler 1 kb DNA ladder and GeneRuler 100 bp DNA ladder (Thermo Fisher Scientific) were used for DNA on-gel band size determination in DNA gel electrophoresis. The PageRuler Prestained Protein Ladder 10-180 kDa (Thermo Fisher Scientific) was used for protein on-gel band size determination. The following named electrophoresis equipment was obtained from BIO-RAD Laboratories (Hercules, CA, USA): DNA agarose gel electrophoresis was performed with Mini-Sub Cell GT chambers and the PowerPac 300 power supply, SDS polyacrylamide gel electrophoresis was conducted with the Mini-Protean Tetra Cell, Mini Trans-Blot Electrophoretic Cell and with the PowerPac 3000 power supply. Amersham Hybond-N nylon membrane for blotting of DNA from agarose gels and Amersham Protran $0.45 \mu \mathrm{m}$ NC nitrocellulose blotting membrane for blotting of proteins from SDS-polyacrylamide gels were purchased from GE Healthcare life sciences (Munich, Germany). The Amersham AlkPhos Direct Labeling and Detection System was used for Southern hybridization analysis. The Amersham Hyperfilm-ECL (GE Healthcare life sciences, Munich, Germany) and the Optimax X-ray Film Processor from Protec GmbH\&Co.KG (Oberstenfeld, Germany) were used for detection of chemiluminescence of Southern and western hybridization. Alternatively, chemiluminescence of western hybridization was visualized with the Fusion SL chemiluminescence detector (PeqLab, Erlangen, Germany). As primary antibodies a-GFP antibody sc-9996 (Santa Cruz Biotechnology, Santa Cruz, CA, USA) and a-HA (clone HA-7; Sigma-Aldrich Chemie GmbH) were used. As secondary antibodies horseradish peroxidase-coupled rabbit G21234 (Invitrogen) and mouse antibody 115-035-003 (Jackson ImmunoResearch, Newmarket, UK) were used.

Pipet tips, reaction tubes, petri dishes, and other plastic consumables were purchased from Sarstedt, StarLab GmbH (Hamburg, Germany), and Nerbe Plus GmbH (Winsen/Luhe, Germany). Centrifugation of $1.5 \mathrm{ml}$ and $2 \mathrm{ml}$ reaction tubes was performed with Heraeus Biofuge Fresco and Heraeus Pico Microcentrifuges from Heraeus Instruments $\mathrm{GmbH}$ (Hanau, Germany). For centrifugation of $15 \mathrm{ml}$ and $50 \mathrm{ml}$ tubes, Centrifuge 5804 R from Eppendorf AG (Hamburg, Germany) was used. The GFL (Burgwedel, Germany) shaking water bath 1086, Orbital shaker 3005, Orbital shaker 3020, and the Rotamax 120 (Heidolph, Schwabach, Germany) were used for incubations at constant agitation.

Plates for phenotypical analysis were scanned via Epson Perfection V600 Photo Scanner (Epson, Suwa, Japan). The binocular microscope SZX12-ILLB2-200 (Olympus Deutschland $\mathrm{GmbH}$, Hamburg, Germany) illuminated with the KL1500-LCD light source (Olympus GmbH, Hamburg, Germany) and the Axiolab light microscope (Zeiss, Oberkochen, Germany) equipped with SC30 cameras (Olympus $\mathrm{GmbH}$ ) and operated 
with the cellSens Dimension version 1.4 software (Olympus $\mathrm{GmbH}$ ) were used for observation of microsclerotia and hypocotyl discolorations of tomato stems.

The confocal Zeiss Observer Z1 microscope (Zeiss) equipped with a CSU-X1 A1 confocal scanner unit (Yokogawa, Ratingen, Germany), QuantEM:512SC digital camera (Photometrics, Tucson, AZ, USA) operated with the SlideBook 6.0 digital microscopy software (Intelligent Imaging Innovations, Goettingen, Germany) was used for fluorescence microscopy. The $\mu$-Slide 8 well (IBIDI, Martinsried, Germany) microscopy chambers were used for localization studies. Vacuoles were visualized in fluorescence microscopy with FM4-64 Dye (Thermo Fisher Scientific).

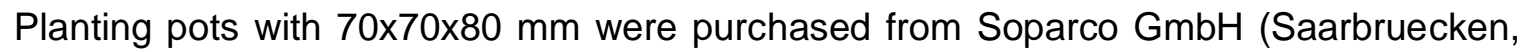
Germany). Fruhstorfer Erde Typ T Struktur 1 -fein- (Archut GmbH\&Co.KG, Lauterbach, Germany) and 0.4-0.8 mm crystal silica sand (Dorsilit, Hirschau, Germany) were used. Plants were grown in a BrightBoy GroBank (CLF PlantClimatics, Emersacker, Germany). Materials and instrumentations, which were not listed here are indicated in the following chapters.

\subsection{Organisms and their cultivation}

All media and supplemental substances used in this study were prepared in deionized water and sterilized either by autoclaving at $121^{\circ} \mathrm{C}$ for $20 \mathrm{~min}$ and 2 bar, or filtersterilized through a $0.2 \mu \mathrm{m}$ pore size membrane filter for temperature unstable substances.

\subsubsection{Cultivation of bacteria}

Escherichia coli and Agrobacterium tumefaciens strains were cultivated using liquid lysogeny broth (LB) medium (Bertani, 1951) [1\% bacto-tryptone, $0.5 \%$ yeast extract, $1 \%$ $\mathrm{NaCl}, \mathrm{pH} 7.5,2 \%$ agar for solid medium] on a rotary shaker at $37^{\circ} \mathrm{C}$ for $E$. coli and $25^{\circ} \mathrm{C}$ for $A$. tumefaciens. Liquid SOC medium ( $2 \%$ bacto tryptone, $0.5 \%$ yeast extract, $10 \mathrm{mM}$ $\mathrm{NaCl}, 2.5 \mathrm{mM} \mathrm{KCl}, 10 \mathrm{mM} \mathrm{MgCl}_{2}, 10 \mathrm{mM} \mathrm{MgSO}_{4}$, supplemented with $20 \mathrm{mM}$ glucose after autoclaving) was used for transformation of chemically competent E. coli and A. tumefaciens cells. Transformants were selected on solid LB medium by supplementation of $100 \mu \mathrm{g} / \mathrm{ml}$ ampicillin or $100 \mu \mathrm{g} / \mathrm{ml}$ kanamycin. For plasmid conservation equal volumes of bacterial overnight cultures and $100 \%$ glycerol were mixed and stored at $-80^{\circ} \mathrm{C}$.

\subsubsection{Cultivation of Verticillium strains}

Conidia were cultivated in liquid simulated xylem medium (SXM; modified from Neumann \& Dobinson, 2003 as described in (Hollensteiner et al., 2017) and in liquid potato dextrose medium (PDM) [2.65\% Potato-Dextrose broth (Carl Roth GmbH\&Co.KG)] and 
incubated at $25^{\circ} \mathrm{C}$ under constant agitation at $120 \mathrm{rpm}$. Mycelium was harvested from PDM liquid cultures for extraction of genomic DNA. Protein extracts were obtained from mycelium harvested from cultures grown in liquid PDM or modified Czapek-Dox Medium (CDM; Smith, 1949) [3\% sucrose, 2\% 50x AspA (3.5 mM NaNO 3,350 mM KCl, 550 mM $\mathrm{KH}_{2} \mathrm{PO}_{4}, \mathrm{pH} 5.5$ ), $2 \mathrm{mM} \mathrm{MgSO}_{4}, 0.001 \% \mathrm{FeSO}_{4}$ ]. Mycelium from liquid SXM or PDM cultures was used for extraction of RNA. Conidia were harvested from SXM cultures by filtration through sterile Miracloth (Calbiochem Merck), washed twice with sterile water, and resuspended in sterile water. The concentrations of spore solutions were determined using the Coulter Particle Count and Size Analyzer Z2 (Beckman Coulter) with the appropriate diluent by counting particles with a size of 1.9-4.5 $\mu \mathrm{m}$ as $V$. dahliae spores or particles with a size of 2.0-5.9 $\mu \mathrm{m}$ for $V$. longisporum spores. For conservation of fungal strains, freshly harvested spores were resuspended in $0.96 \% \mathrm{NaCl}$ with $0.05 \%$ Tween80, adjusted to $2 \times 10^{7}$ spores $/ \mathrm{ml}$ and preserved as $30 \%$ glycerol stock aliquots at $80^{\circ} \mathrm{C}$.

Verticillium transformants were selected on solid potato dextrose medium (PDM, 3.9\% potato extract glucose agar (Carl Roth GmbH\&Co.KG), 0.5\% agar) supplemented with antimycotics clonNAT nourseothricin dihydrogen sulfate $(72 \mu \mathrm{g} / \mathrm{ml}$, Werner Bioagents $\mathrm{GmbH})$ or hygromycin B (50 $\mu \mathrm{g} / \mathrm{ml}$, InvivoGen), and the antibiotic cefotaxime (300 $\mu \mathrm{g} / \mathrm{ml}$; Wako Chemicals $\mathrm{GmbH}$ ) after autoclaving.

\subsection{Strains, plasmids and primers}

\subsubsection{Bacterial strains}

E. coli strain DH5a (Woodcock et al., 1989) was used for cloning, plasmid reproduction, and conservation. A.tumefaciens strain AGL-1 (Lazo et al., 1991) was used for A. tumefaciens-mediated transformation (ATMT) for generation of different $V$. dahliae and $V$. longisporum strains.

\subsubsection{Verticillium strains}

The $V$. longisporum strain VI43 isolated from Brassica napus (Zeise \& von Tiedemann, 2002) was used as parental lineage for the generation of $V$. longisporum strains. The V. dahliae strain JR2 (Fradin et al., 2009) isolated from Solanum lycopersicum was used for the generation of $V$. dahliae deletion strains in this study. Fungal strains constructed and used in this study are listed in Table 1. 
Table 1: Verticillium strains constructed and used in this study.

\begin{tabular}{|c|c|c|}
\hline Strain & Description & Reference \\
\hline \multicolumn{3}{|c|}{ V. dahliae strains } \\
\hline JR2/ WT & Solanum lycopersicum isolate & $\begin{array}{l}\text { (Fradin et al., } \\
2009)\end{array}$ \\
\hline JR2-GFP & ${ }^{p}$ gpdA:GFP:trpC ${ }^{t} \cdot p$ gpdA:HYG ${ }^{R}: \operatorname{trp} C^{t}$ & (Bui et al., 2019) \\
\hline VGB392 & ${ }^{p}$ gpdA:GFP:trp $C^{t} \cdot \mathrm{g} g p d A: N A T^{R}: \operatorname{trp} C^{t}$ & This study \\
\hline $\begin{array}{l}\text { VGB279/ } \\
\text { VGB280 }\end{array}$ & $\triangle H A M 5::^{p} g p d A: N A T^{R}: \operatorname{trp} C^{t}$ & This study \\
\hline $\begin{array}{l}\text { VGB331/ } \\
\text { VGB332 }\end{array}$ & $\triangle O D E 1::^{p} g p d A: N A T^{R}: \operatorname{trp} C^{t}$ & This study \\
\hline $\begin{array}{l}\text { VGB335/ } \\
\text { VGB336 }\end{array}$ & $\Delta V M K 1::^{p} g p d A: H Y G^{R}: \operatorname{trp} C^{t}$ & This study \\
\hline $\begin{array}{l}\text { VGB337/ } \\
\text { VGB338 }\end{array}$ & $\triangle M E K 2::^{p} g p d A: H Y G^{R}: \operatorname{trp} C^{t}$ & This study \\
\hline VGB346 & $\begin{array}{l}\triangle M E K 2::^{p} g p d A: H Y G^{R}: \operatorname{trp} C^{t} \\
\Delta H A M 5::^{p} g p d A: N A T^{R}: \operatorname{trp} C^{t}\end{array}$ & This study \\
\hline $\begin{array}{l}\text { VGB358/ } \\
\text { VGB359 }\end{array}$ & 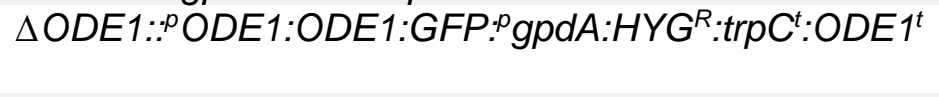 & This study \\
\hline $\begin{array}{l}\text { VGB360/ } \\
\text { VGB361 }\end{array}$ & $\triangle O D E 1::{ }^{p} O D E 1: G F P: O D E 1:^{p} g p d A: H Y G^{R}: \operatorname{trp} C^{t}: O D E 1^{t}$ & This study \\
\hline $\begin{array}{l}\text { VGB371/ } \\
\text { VGB372 }\end{array}$ & $\triangle H A C 1::^{p} g p d A: H Y G^{R}: \operatorname{trp} C^{t}$ & This study \\
\hline VGB380 & $\begin{array}{l}\Delta H A C 1::^{p} g p d A: H Y G^{R}: \operatorname{trp} C^{t} \\
{ }^{p} \text { gpdA:GFP:trp } C^{t}:{ }^{p} g p d A: N A T^{R}: \operatorname{trp} C^{t}\end{array}$ & This study \\
\hline VGB382 & $\begin{array}{l}\text { DHAC1::pgpdA:HYG }{ }^{R}: \operatorname{trp}^{t} \\
{ }^{p} H A C 1: H A C 1: H A C 1^{t}:{ }^{p} g p d A: N A T^{R}: \operatorname{trp} C^{t}\end{array}$ & This study \\
\hline $\begin{array}{l}\text { VGB383/ } \\
\text { VGB393 }\end{array}$ & Vd JR2 $2 L S::{ }^{p} g p d A: N A T^{R}: \operatorname{trp} C^{t}$ & This study \\
\hline $\begin{array}{l}\text { VGB388/ } \\
\text { VGB389 }\end{array}$ & 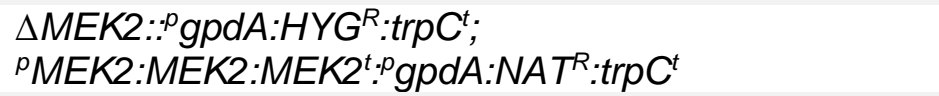 & This study \\
\hline VGB413 & $\begin{array}{l}\Delta V M K 1::^{p} g p d A: H Y G^{R}: \operatorname{trp} C^{t} \\
{ }^{p} V M K 1: V M K 1: V M K 1^{t}:{ }^{p} g p d A: N A T^{R}: \operatorname{trp} C^{t}\end{array}$ & This study \\
\hline VGB415 & $\begin{array}{l}\text { DHAM5::pgpdA:NAT }: \operatorname{trp}^{t} ; \\
{ }^{p} H A M 5: H A M 5: H A M 5^{t}:{ }^{p} g p d A: H Y G^{R}: \operatorname{trp} C^{t}\end{array}$ & This study \\
\hline VGB417 & $\begin{array}{l}\Delta V M K 1::^{p} g p d A: H Y G^{R}: \operatorname{trp} C^{t} \\
\Delta H A M 5::^{p} g p d A: N A T^{R}: \operatorname{trp} C^{t}\end{array}$ & This study \\
\hline $\begin{array}{l}\text { VGB439/ } \\
\text { VGB440 }\end{array}$ & $\begin{array}{l}\Delta H A C 1::^{p} \text { gpdA:HYGR }: \operatorname{trp}^{t} \text {; } \\
{ }^{p} H A C 1: H A C 1^{u}: H A C 1^{t}: p g p d A: N A T^{R}: \operatorname{trpC}^{t}\end{array}$ & This study \\
\hline $\begin{array}{l}\text { VGB437/ } \\
\text { VGB438 }\end{array}$ & $\begin{array}{l}\triangle H A C 1::^{\circ} \text { gpdA:HYG }{ }^{R}: \operatorname{trp} C^{t} ; \\
{ }^{p} H A C 1: H A C 1^{i}: H A C 1^{t} \cdot{ }^{p} g p d A: N A T^{R}: \operatorname{trp} C^{t}\end{array}$ & This study \\
\hline \multicolumn{3}{|c|}{ V. longisporum strains } \\
\hline VI32 & B. napus (oilseed rape) isolate & $\begin{array}{l}\text { (Zeise \& von } \\
\text { Tiedemann, 2002) }\end{array}$ \\
\hline V143 & B. napus (oilseed rape) isolate & $\begin{array}{l}\text { (Zeise \& von } \\
\text { Tiedemann, 2002) }\end{array}$ \\
\hline $\begin{array}{l}\text { VGB193/ } \\
\text { VGB214 }\end{array}$ & $\begin{array}{l}\text { VI43ALSII::p gpdA:NATR }: \operatorname{trp}^{t} \\
(\Delta \text { vI43ass51scaf493:534161-542607) }\end{array}$ & This study \\
\hline $\begin{array}{l}\text { VGB201/ } \\
\text { VGB233 }\end{array}$ & 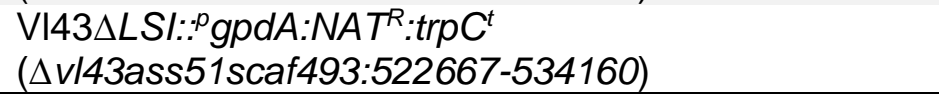 & This study \\
\hline
\end{tabular}




\begin{tabular}{|c|c|c|c|}
\hline Strain & \multicolumn{2}{|c|}{ Description } & Reference \\
\hline VGB232 & \multicolumn{2}{|c|}{$\begin{array}{l}\text { VI43ALS::pgpdA:HYG }{ }^{R}: \operatorname{trp} C^{t} \\
(\Delta v 143 \text { ass51scaf493:522667-542607) }\end{array}$} & This study \\
\hline \multicolumn{4}{|c|}{$\begin{array}{l}\text { p: promoter, } t \text { : terminator, } N A T^{R}: \text { nourseothricin resistance marker, } H Y G^{R} \text { : hygromycin } \mathrm{B} \\
\text { resistance marker, two VGB numbers for one genotype indicate two independen } \\
\text { transformants. }\end{array}$} \\
\hline \multicolumn{4}{|c|}{ Plants } \\
\hline \multicolumn{4}{|c|}{ All plants used in this study were purchased as seeds and are listed in Table 2.} \\
\hline Organism & & Description & Reference \\
\hline $\begin{array}{l}\text { Brassica nap } \\
\text { (falcon) }\end{array}$ & & $\begin{array}{l}\text { rapeseed, family: Brassicaceae, used } \\
\text { as host for } V \text {. longisporum }\end{array}$ & $\begin{array}{l}\text { Norddeutsche Pflanzenzucht } \\
\text { (Holtsee, Germany) }\end{array}$ \\
\hline $\begin{array}{l}\text { Solanum } \\
\text { lycopersicum } \\
\text { (moneymake }\end{array}$ & & $\begin{array}{l}\text { tomato, family: Solanaceae, used as } \\
\text { host for } V \text {. dahliae }\end{array}$ & $\begin{array}{l}\text { Bruno Nebelung GmbH\&Co. } \\
\text { Kiepenkerl-Pflanzenzüchtung } \\
\text { (Everswinkel, Germany) }\end{array}$ \\
\hline $\begin{array}{l}\text { Arabidopsis } \\
\text { thaliana } \\
\text { (Col-0) }\end{array}$ & & $\begin{array}{l}\text { thale cress, family: Brassicaceae, } \\
\text { used for root colonization analysis by } \\
V \text {. longisporum and } V \text {. dahliae }\end{array}$ & $\begin{array}{l}\text { Nottingham Arabidopsis } \\
\text { Stock Centre, stock no. } \\
\text { N1902 }\end{array}$ \\
\hline
\end{tabular}

\subsubsection{Primers, Plasmid, and strain constructions}

All fragments were PCR amplified using the Q5 Hot Start polymerase (New England Biolabs) or Phusion High-Fidelity DNA polymerase (Thermo Fisher Scientific) from given templates. All strains, primers, and plasmids are listed in Table 1, 3, and 4.

\subsubsection{Primers}

Oligonucleotides used in this study are listed in Table 4. Annealing temperatures of primers were calculated using the NEB Tm Calculator v1.9.13 and the Eurofins Genomics Oligo analysis tool for qPCR primers. Primers used in this study are listed in Table 3.

Table 3: Primers used and designed in this study.

\begin{tabular}{llll}
\hline $\begin{array}{l}\text { Primer } \\
\text { name }\end{array}$ & Primer sequence $\left(\mathbf{5}^{\prime} \boldsymbol{\rightarrow} \mathbf{3}^{\prime} \mathbf{)}\right.$ & $\begin{array}{l}\text { Length } \\
(\mathbf{b p})\end{array}$ & $\begin{array}{l}\text { Overhang } \\
\text { to }\end{array}$ \\
\hline AO144 & AAT AAT CAT CCA CTG CAC CT & 20 & - \\
JST1 & GTA TGT TGT GTG GAA GCT ACC TAC CGA CAT GCC & 35 & pME4564 \\
& CG & & \\
JST2 & ACC GGT CAC TGT ACA TGC TGT GAC CGT TCG TTT & 36 & ${ }^{p} g p d A$ \\
& TCC & & \\
JST3 & AGG TAA TCC TTC TTT CCA TAT TCT GAT GGT TTC & 33 & trpC \\
JST4 & CAC AGT ACA CGA GGA TAC AGA TCT TAT AGA ACT & 37 & pME4564 \\
& GTA C & & \\
\hline
\end{tabular}




\begin{tabular}{|c|c|c|c|}
\hline $\begin{array}{l}\text { Primer } \\
\text { name }\end{array}$ & Primer sequence $\left(5^{\prime} \rightarrow 3^{\prime}\right)$ & $\begin{array}{l}\text { Length } \\
\text { (bp) }\end{array}$ & $\begin{array}{l}\text { Overhang } \\
\text { to }\end{array}$ \\
\hline JST5 & $\begin{array}{l}\text { GTA TGT TGT GTG GAA CGG GTG CGA CAA ATG CTC } \\
\text { TC }\end{array}$ & 35 & pME4564 \\
\hline JST6 & ACC GGT CAC TGT ACA GCC TGC TGT CCG TGG CAC & 33 & ${ }^{p}$ gpdA \\
\hline JST7 & AGG TAA TCC TTC TTT GTC AGC CCG GAG ACG GTG & 33 & $\operatorname{trp} C^{t}$ \\
\hline JST8 & $\begin{array}{l}\text { CAC AGT ACA CGA GGA CGC CCA GAA ACC TGA CAA } \\
\text { CC }\end{array}$ & 35 & pME4564 \\
\hline JST9 & $\begin{array}{l}\text { AGA TCC CCG GGT ACC TGC TGT GAC CGT TCG TTT } \\
\text { TCC }\end{array}$ & 36 & ${ }^{p} g p d A$ \\
\hline JST11 & CCA TGG AGC ATA CAA ATA GCT & 21 & - \\
\hline JST13 & TCC CAC TGT AGG ACC AGA G & 19 & - \\
\hline JST15 & CGT CAG GGT AGC ATC ATT C & 19 & - \\
\hline JST16 & GAC CTG ATC GGA TGA CAT GC & 20 & - \\
\hline JST17 & CAC TCT CGA CTG CCG CCT G & 19 & - \\
\hline JST21 & $\begin{array}{l}\text { AGG TAA TCC TTC TTT TTC CTT ATG TTG GGA TTT } \\
\text { GAG G }\end{array}$ & 37 & $\operatorname{trp} C^{t}$ \\
\hline JST45 & TCA GAT CAT CTC GCT TTC GTG T & 22 & - \\
\hline JST46 & TCA GAC GCC TTC CGT GTT GG & 20 & - \\
\hline JST50 & ATG ACC TCG GAC CAC CAT TT & 20 & - \\
\hline JST53 & ATG ACC GGC GAC GAA GTA GC & 20 & - \\
\hline JST58 & CGG TCA CAA GAG CGT CTG GA & 20 & - \\
\hline JST61 & ATG TCG CGC AGC AAC GC & 17 & - \\
\hline JST62 & CTA CCG CAT AAT CTC CTG GTA GA & 23 & - \\
\hline JST76b & GTA TGT TGT GTG GAA AGC ACG GAG CAG AGA CCA & 33 & $\mathrm{pPK} 2$ \\
\hline JST77a & GGT TCT GGT ACA CGA CGA GC & 20 & - \\
\hline JST90 & GGC TAG GCG ACA TTG ACC CTG & 21 & - \\
\hline JST110 & $\begin{array}{l}\text { GTA TGT TGT GTG GAA CCG CGA GGG TTG GAG } \\
\text { AGG }\end{array}$ & 33 & pME4564 \\
\hline JST111 & $\begin{array}{l}\text { ACC GGT CAC TGT ACA GAC GGG CCT GAT ATT CTT } \\
\text { TCG A }\end{array}$ & 37 & ${ }^{p} g p d A$ \\
\hline JST112 & $\begin{array}{l}\text { AGG TAA TCC TTC TTT GTG GCC GTC TTT TCA CAG } \\
\text { GC }\end{array}$ & 35 & $\operatorname{trp} C^{t}$ \\
\hline JST113 & $\begin{array}{l}\text { CAC AGT ACA CGA GGA TCT CGT CCG GAC TGA TCC } \\
\text { AA }\end{array}$ & 35 & pME4564 \\
\hline JST127 & $\begin{array}{l}\text { CGT ATG TTG TGT GGA AGG ATG GCC AAT GTG GAT } \\
\text { TTG AT }\end{array}$ & 38 & pME4564 \\
\hline JST128 & $\begin{array}{l}\text { CAC CGG TCA CTG TAC AGG TAC TGG TGG CTC TTG } \\
\text { GGA }\end{array}$ & 36 & ${ }^{p} g p d A$ \\
\hline JST129 & $\begin{array}{l}\text { GAG GTA ATC CTT CTT TTC GGA TTG GAC AGT AGA } \\
\text { CAA GTT TG }\end{array}$ & 41 & ${ }^{p} g p d A$ \\
\hline JST130 & $\begin{array}{l}\text { CAC AGT ACA CGA GGA CGC GCA CAG TTA CAC TTC } \\
\text { ATA CTC T }\end{array}$ & 40 & pME4564 \\
\hline JST137 & $\begin{array}{l}\text { TCC ACA TTG GCC ATC CTT CCA CAC AAC ATA CGA } \\
\text { GCC G }\end{array}$ & 37 & ${ }^{\mathrm{P}} O D E 1$ \\
\hline JST138 & $\begin{array}{l}\text { AGT GTA ACT GTG CGC GTC CTC GTG TAC TGT GTA } \\
\text { AGC }\end{array}$ & 36 & $O D E 1^{t}$ \\
\hline JST171 & ATG GAG TCT TGG GAG CAC TC & 20 & - \\
\hline JST172 & TCA GAC ACC AAC CGC AAT & 18 & - \\
\hline JST174 & TCA GCG AAA GCG CAC TC & 17 & - \\
\hline JST175 & ATG GCT GCG ACC ACA TC & 17 & - \\
\hline
\end{tabular}




\begin{tabular}{|c|c|c|c|}
\hline $\begin{array}{l}\text { Primer } \\
\text { name }\end{array}$ & Primer sequence $\left(5^{\prime} \rightarrow 3^{\prime}\right)$ & $\begin{array}{l}\text { Length } \\
\text { (bp) }\end{array}$ & $\begin{array}{l}\text { Overhang } \\
\text { to }\end{array}$ \\
\hline JST176 & TCA CTG CTC ATC CGT ACG & 18 & - \\
\hline JST177 & AAA GAA GGA TTA CCT CTA AAC AAG TGT & 27 & - \\
\hline JST178 & $\begin{array}{l}\text { GGT ACC GAG CTC GAT TTA CTT GTA CAG CTC GTC } \\
\text { CA }\end{array}$ & 35 & ${ }^{p}$ gpdA \\
\hline JST179 & ACC ACC GCT ACC ACC CTG CTC ATC CGT ACG GC & 32 & $\begin{array}{l}\text { linker C- } \\
\text { terminal } \\
\text { GFP }\end{array}$ \\
\hline JST180 & ATT CTT AAT TAA GAT GGA TGG CCA ATG TGG AT & 32 & pPK2 \\
\hline JST181 & GCC CTT GCT CAC CAT GGT ACT GGT GGC TCT TGG & 33 & $\begin{array}{l}\text { N-terminal } \\
\text { GFP }\end{array}$ \\
\hline JST184 & GTA TGT TGT GTG GAA CGA GTG GAG ATG TGG AGT & 33 & pME4815 \\
\hline JST185 & ACC GGT CAC TGT ACA TGG CAT GCG GAG AGA C & 31 & pME4815 \\
\hline JST186 & ATT CTT AAT TAA GAT GAC AAG AGT CAA GCC CAC & 33 & pME4564 \\
\hline JST187 & AGA TCC CCG GGT ACC GAT GGA CGA AGC GAC TC & 32 & ${ }^{p} g p d A$ \\
\hline JST188 & $\begin{array}{l}\text { AGG TAA TCC TTC TTT TTT GAT TTT TAT CAT GAT } \\
\text { GAC GGC }\end{array}$ & 39 & $\operatorname{trp} C^{t}$ \\
\hline JST189 & $\begin{array}{l}\text { AGG ACT TCT AGA AGG TCC AGC TCC AAA TCA ATT } \\
\text { AAC C }\end{array}$ & 37 & pME4564 \\
\hline JST207 & TCA GTG GTT TTC GAT AGA CG & 20 & - \\
\hline JST208 & ATG ACA ACC AAC TCA TCT GA & 20 & - \\
\hline JST210 & $\begin{array}{l}\text { GTA TGT TGT GTG GAA GCT ACC TAC CGA CAC GCC } \\
\text { C }\end{array}$ & 34 & pME4564 \\
\hline JST211 & $\begin{array}{l}\text { GGT CAC TGT ACA GAT GGG ACT CGT ACC ATG TTT } \\
\text { C }\end{array}$ & 34 & $\operatorname{trp} C^{t}$ \\
\hline JST212 & $\begin{array}{l}\text { TGT TGT GTG GAA GAT ACT AAG TAC TGG TTG TGG } \\
\text { CTG AC }\end{array}$ & 38 & pME4815 \\
\hline JST213 & GGT CAC TGT ACA GAT AGG CTT GGA GAT GAC GAG & 33 & ${ }^{p} g p d A$ \\
\hline JST216 & TGT TGT GTG GAA GAT GAC AAG AGT CAA GCC CAC & 33 & pME4564 \\
\hline JST217 & TCA TTT TTC CAC СTT TCT CA & 20 & - \\
\hline JST218 & ATG TAC CCA GCA AAC TAC GA & 20 & - \\
\hline JST220 & TGG AAG ATT AGG ACA TGA & 18 & - \\
\hline JST223 & ATA GGA CAT TAT CAT CGC & 18 & - \\
\hline JST224 & GCA GCA AGT TCA CTC GAG & 18 & - \\
\hline JST226 & ATG CCC GAT CCT TTC GC & 17 & - \\
\hline JST228 & AGG GTG CGA TGC GTT GTT & 18 & - \\
\hline JST229 & TTA TGG CTT CGA CCA GTA TGG & 21 & - \\
\hline JST230 & ATG CTG ACT TCG GCC CCT & 18 & - \\
\hline JST231 & CTA CAT GTA GTC TTT ATT TTG ATG C & 25 & - \\
\hline JST232 & ATG TCC TCC AAC ATC CC & 17 & - \\
\hline JST233 & TCA GAC AAC TTC GCC GTG G & 19 & - \\
\hline JST234 & ATG TCC CGC CGT CTC CTG & 18 & - \\
\hline JST235 & CTA CCC GCA GGG GGT GC & 17 & - \\
\hline JST236 & ATG CAC TTT CAC GAA ATG GCT C & 22 & - \\
\hline JST238 & ACT TCG CGC AGT ACG ACT & 18 & - \\
\hline JST239 & AGG TAC TGG TGA AGG CAT & 18 & - \\
\hline JST240 & TCA GAG AAT GTG AAG ATG & 18 & - \\
\hline JST241 & CTT CGT CTT CTG TTT TCG & 18 & - \\
\hline
\end{tabular}




\begin{tabular}{|c|c|c|c|}
\hline $\begin{array}{l}\text { Primer } \\
\text { name }\end{array}$ & Primer sequence $\left(5^{\prime} \rightarrow 3^{\prime}\right)$ & $\begin{array}{l}\text { Length } \\
\text { (bp) }\end{array}$ & $\begin{array}{l}\text { Overhang } \\
\text { to }\end{array}$ \\
\hline JST242 & GAG ATC GAC AAC GCT GTC & 18 & - \\
\hline JST243 & TGT TGT GTG GAA GAT AGC ACG GAG CAG AGA CCA & 33 & pME4815 \\
\hline JST244 & $\begin{array}{l}\text { GGT CAC TGT ACA GAT CGA ACC GGT GAT GGA TAC } \\
\text { G }\end{array}$ & 34 & ${ }^{p}$ gpdA \\
\hline JST245 & ATT CTT AAT TAA GAT CTG CTC CTA TTC GGC TCC & 33 & pPK2 \\
\hline JST246 & GGT ACC GAG CTC GAT TCT CGT CCG GAC TGA TCC & 33 & pPK2 \\
\hline JST266 & $\begin{array}{l}\text { CTA TCC GCC GCT AGC GTA ATC GGG CAC ATC GTA } \\
\text { TGG GTA GCC GCC GCT GAC ACC AAC CGC AAT GC }\end{array}$ & 65 & $H A$ tag \\
\hline JST267 & $\begin{array}{l}\text { CTA TCC GCC GCT AGC GTA ATC GGG CAC ATC GTA } \\
\text { TGG GTA GCC GCC GCT GCG AAA GCG CAC TCG T }\end{array}$ & 64 & $H A$ tag \\
\hline JST268 & CTA TCC GCC GCT AGC GTA & 18 & - \\
\hline JST269 & TGT TGT GTG GAA GAT GCT GAG GTC ATG GCT GAC & 33 & pME4564 \\
\hline JST270 & CTC CCA AGA CTC CAT TTT GGA CGG CTT TGT GTG & 33 & HAC1 \\
\hline JST271 & $\begin{array}{l}\text { GCT AGC GGC GGA TAG GGG CTG TGA GAA TCG } \\
\text { GGT }\end{array}$ & 33 & $H A$ \\
\hline JST272 & $\begin{array}{l}\text { GGT CAC TGT ACA GAT GGG ACT CGT ACC ATG TTT } \\
\text { CA }\end{array}$ & 35 & $\operatorname{trp} C^{t}$ \\
\hline JST273 & $\begin{array}{l}\text { GCT AGC GGC GGA TAG TTT GAT TTT TAT CAT GAT } \\
\text { GAC GG }\end{array}$ & 38 & $H A$ \\
\hline JST288 & AGG TGA GAA CCG AGC AC & 17 & - \\
\hline JST289 & AGT GCA TTC TTG TGA AGC C & 19 & - \\
\hline JST292 & TTA ATT CGA GGA CCC ATT GTA AA & 23 & - \\
\hline JST293 & CCG ACG TGA CAC AAC GTC & 18 & - \\
\hline JS-V5 & $\begin{array}{l}\text { CGT ATG TTG TGT GGA AAC TAA GTA CTG GTT GTG } \\
\text { GCT GAC }\end{array}$ & 39 & $\mathrm{pPK} 2$ \\
\hline JS-V6 & $\begin{array}{l}\text { AAG ATC CCC GGG TAC CTT TGG GTG ATG TGC GTG } \\
\text { G }\end{array}$ & 34 & ${ }^{P}$ gpdA \\
\hline JS-V7 & $\begin{array}{l}\text { ACA ACC AGT ACT TAG TTT CCA CAC AAC ATA CGA } \\
\text { G }\end{array}$ & 34 & pMEK2 \\
\hline JS-V8 & $\begin{array}{l}\text { ACG CAC ATC ACC CAA AGG TAC CCG GGG ATC TTT } \\
\text { C }\end{array}$ & 34 & ${ }^{p} M E K 2$ \\
\hline JS-V9 & $\begin{array}{l}\text { TCC TTC TTT CTA GAA GTT GAA CAG GCC TGT CTG } \\
\text { G }\end{array}$ & 34 & pME4821 \\
\hline JS-V10 & $\begin{array}{l}\text { ACA CAG TAC ACG AGG AAG GCT TGG AGA TGA CGA } \\
\text { G }\end{array}$ & 34 & pME4821 \\
\hline JS-V11 & $\begin{array}{l}\text { CGT CAT CTC CAA GCC TTC CTC GTG TAC TGT GTA } \\
\text { AG }\end{array}$ & 35 & $M E K 2^{t}$ \\
\hline JS-V12 & $\begin{array}{l}\text { AGA CAG GCC TGT TCA ACT TCT AGA AAG AAG GAT } \\
\text { TAC CTC }\end{array}$ & 39 & $M E K 2^{t}$ \\
\hline JS-V21 & GAG GTA ATC CTT CTT TGG TGG CAG TGG CAG TGG & 33 & pPK2 \\
\hline JS-V22 & $\begin{array}{l}\text { ACA CGA GGA CTT CTA GCG AAC CGG TGA TGG ATA } \\
\text { CGT T }\end{array}$ & 37 & $\mathrm{pPK} 2$ \\
\hline JS-V23 & $\begin{array}{l}\text { ATC CAT CAC CGG TTC GCT AGA AGT CCT CGT GTA } \\
\text { CTG T }\end{array}$ & 37 & $V M K 1^{t}$ \\
\hline JS-V24 & $\begin{array}{l}\text { CAC TGC CAC TGC CAC CAA AGA AGG ATT ACC TCT } \\
\text { AAA CAA GT }\end{array}$ & 41 & $V M K 1^{t}$ \\
\hline ML1 & TTC CAC ACA ACA TAC GAG CC & 20 & - \\
\hline ML2 & TCC TCG TGT ACT GTG TAA GC & 20 & - \\
\hline ML5 & TGT ACA GTG ACC GGT GAC TCT T & 22 & - \\
\hline
\end{tabular}




\begin{tabular}{llll}
\hline $\begin{array}{l}\text { Primer } \\
\text { name }\end{array}$ & Primer sequence $\left(\mathbf{5}^{\prime} \rightarrow \mathbf{3}^{\prime} \mathbf{)}\right.$ & $\begin{array}{l}\text { Length } \\
(\mathbf{b p})\end{array}$ & $\begin{array}{l}\text { Overhang } \\
\text { to }\end{array}$ \\
\hline ML6 & TCC CGC GGT CGG CAT CTA CTT CAG GGG CAG & 38 & trpC $^{t}$ \\
ML8 & GGC ATG CT & 23 & - \\
ML9 & TGT ACA GTG ACC GGT GAC & 18 & - \\
pKO2- & TGT GGA CAA AGG ATT ACG GG & 20 & - \\
40B-for & & & \\
RO3 & GGT ACC CGG GGA TCT TTC G & 19 & - \\
RO4 & AAA GAA GGA TTA CCT CTA AAC AA & 23 & - \\
SAB16 & GGT GGT AGC GGT GGT ATG & 18 & - \\
SZ9 & AAC ACC CAG AAC AAG ATG CGC & 21 & - \\
SZ10 & GCT TGA CCT TGA GAT CCT TG & 20 & - \\
SZ11 & TGC ATT CTT GGC AAG AGA TGT GTG & 24 & - \\
SZ12 & AGC TTG TTA TCC TTG TCC TCG GT & 23 & - \\
SZ19 & ACC TCT GGA GGC AAG GCT T & 19 & - \\
SZ20 & GCT TGG CCT TCT TCT TCT GC & 20 & - \\
ZQY11 & GCA TGG ACG AGC TGT ACA AGG GTG GTA GCG & 35 & fusion to N- \\
& GTG GT & & terminal \\
& & & GFP \\
\hline
\end{tabular}

Blue: overhangs for Seamless and FastCloning, purple: overhang for fusion of linker or tag, ${ }^{p}$ : promoter, ${ }^{t}$ : terminator.

\subsubsection{Plasmids}

For construction of deletion and complementation cassettes, the GeneArt Seamless Cloning and Assembly Kit (Thermo Fisher Scientific) and the FastCloning protocol ( $\mathrm{Li}$ et al., 2011) were used. Therefore, 1-2 kb flanking sequences up- and downstream of the corresponding gene or region were amplified from genomic V. Iongisporum VI43 wild type DNA with 15-16 bp homologous overhangs to the desired neighboring fragments. The PCR products were purified with NucleoSpin Gel and PCR Clean-up Kit (MachereyNagel) and used for transformation of E. coli. All plasmids were verified by Sanger sequencing.

For construction of Verticillium strains, the nourseothricin resistance marker $\left(N A T^{R}\right)$ and the hygromycin B resistance marker $\left(H Y G^{R}\right)$ were used for selection of transformants. Both resistance markers are flanked by the $A$. nidulans glyceraldehyde-3-phosphate dehydrogenase gpdA (AN8041; A. nidulans FGSC A4) promoter (Punt et al., 1988; David et al., 2008) and the tryptophan biosynthesis gene trpC (AN0648; A. nidulans FGSC A4) terminator for constitutive heterologous gene expression and are named $N A T^{R}$ and $H Y G^{R}$ marker cassettes in the following. The same promoter and terminator were used for ectopic overexpression of GFP in Verticillium strains.

Plasmids constructed and used in this study are listed in Table 4. 
Table 4: Plasmids constructed and used in this study.

\begin{tabular}{|c|c|c|}
\hline Plasmid & Description & Reference \\
\hline pGreen2 & $\begin{array}{l}{ }^{p} \text { gpdA:HYG }{ }^{R}: \operatorname{trp} C^{t} ;{ }^{p} g p d A: G F P: \operatorname{trp} C^{t} ; K A N^{R} \text {, } \\
\text { left and right border for ATMT }\end{array}$ & (Tran et al., 2014) \\
\hline pJet1.2 & Cloning vector with $A M P^{R}$ & $\begin{array}{l}\text { Thermo Fisher } \\
\text { Scientific }\end{array}$ \\
\hline pPK2 & $\begin{array}{l}\text { Cloning vector with } K A N^{R} \text { and } H Y G^{R} \text {, } \\
\text { left and right border for ATMT }\end{array}$ & (Covert et al., 2001) \\
\hline pME4564 & $\begin{array}{l}\text { Cloning vector with } K A N^{R} \text { and } H Y G^{R} \text {, } \\
\text { left and right border for ATMT }\end{array}$ & $\begin{array}{l}\text { personal } \\
\text { communication Van } \\
\text { Tuan Tran, University } \\
\text { of Goettingen }\end{array}$ \\
\hline pME4815 & $\begin{array}{l}\text { Cloning vector with } K A N^{R} \text { and } N A T^{R}, \\
\text { left and right border for ATMT } \\
\left({ }^{p} g p d A: N A T^{R}: t r p C^{t} \text { in pME4564) }\right.\end{array}$ & $\begin{array}{l}\text { (Balnojan, 2016, MT, } \\
\text { University of } \\
\text { Goettingen) }\end{array}$ \\
\hline pME4816 & pVI43-LSI: ${ }^{p} g p d A: N A T^{R}: \operatorname{trp}^{t}: L S I^{\prime}$ in pME4564 & This study \\
\hline pME4817 & ${ }^{p} \mathrm{VI} 43-L S I I:^{p} g p d A: N A T^{R}: \operatorname{trp}^{t}: L S I I^{t}$ in pME4564 & This study \\
\hline pME4818 & ${ }^{p} \mathrm{VI} 43-L S::^{. p} g p d A: H Y G^{R}: \operatorname{trp} C^{t}: L S^{t}$ in pPK2 & This study \\
\hline pME4819 & ${ }^{p}$ gpdA:GFP:trp $C^{t}$ in pME4815 & This study \\
\hline pME4820 & ${ }^{p} H A M 5:^{p} g p d A: N A T^{R}: \operatorname{trp}^{t}:$ HAM5 ${ }^{t}$ in pME4564 & This study \\
\hline pME4821 & ${ }^{P} M E K 2$ in $\mathrm{pPK} 2$ & This study \\
\hline pME4822 & ${ }^{p} M E K 2{ }^{p} g p d A: H Y G^{R}: \operatorname{trp} C^{t}: M E K 2^{t}$ in pPK2 & This study \\
\hline pME4824 & $V M K 1^{t}$ in $\mathrm{pPK} 2$ & This study \\
\hline pME4825 & ${ }^{p} V M K 1:^{p} g p d A: H Y G^{R}: \operatorname{trp} C^{t}: V M K 1^{t}$ in pPK2 & This study \\
\hline pME4826 & ${ }^{p} M E K 2: M E K 2: M E K 2^{t}$ in pME4815 & This study \\
\hline pME4827 & ${ }^{p} V M K 1: V M K 1: V M K 1^{t}$ in pME4815 & This study \\
\hline pME4828 & ${ }^{p} H A M 5: H A M 5: H A M 5^{t}: p g p d A: H Y G^{R}: \operatorname{trp} C^{t}$ in pPK2 & This study \\
\hline pME4829 & ${ }^{p}$ VdJR2-LS: ${ }^{p} g p d A: N A T^{R}: \operatorname{trp}^{t}: L S^{t}$ in pME4564 & This study \\
\hline pME4830 & ${ }^{p} H A C 1: P g p d A: H Y G^{R}: \operatorname{trp} C^{t}: H A C 1^{t}$ in pPK2 & This study \\
\hline pME4831 & ${ }^{p} H A C 1: H A C 1: H A C 1^{t}$ in pME4815 & This study \\
\hline pME4832 & $H A C 1^{u}: H A$ in pJet1.2 & This study \\
\hline pME4833 & $H A C 1^{i}: H A$ in pJet1.2 & This study \\
\hline pME4834 & ${ }^{p} H A C 1: H A C 1^{i}: H A C 1^{t}$ in pME4564 & This study \\
\hline pME4835 & ${ }^{P} H A C 1: H A C 1^{u}: H A C 1^{t}$ in pME4564 & This study \\
\hline pME4836 & ${ }^{P} O D E 1:^{p} g p d A: N A T^{R}: \operatorname{trp} C^{t}: O D E 1^{t}$ in pME4564 & This study \\
\hline pME4837 & $O D E 1^{t}$ in $\mathrm{pPK} 2$ & This study \\
\hline pME4838 & $\begin{array}{l}{ }^{p} O D E 1: O D E 1: G F P{ }^{p} g p d A: H Y G^{R}: \operatorname{trp} C^{t}: O D E 1^{t} \text { in } \\
\text { pME4837 }\end{array}$ & This study \\
\hline pME4839 & $\begin{array}{l}\text { PODE1:GFP:ODE1: }{ }^{p} g p d A: H Y G^{R}: \operatorname{trp} C^{t}: O D E 1^{t} \text { in } \\
\text { pME4837 }\end{array}$ & This study \\
\hline
\end{tabular}

$A M P^{R ;}$ ampicillin resistance marker, ATMT: Agrobacterium tumefaciens-mediated transformation, $H Y G^{R}$ : hygromycin $\mathrm{B}$ resistance marker, $K A N^{R}$ : kanamycin resistance marker, $N A T^{R}$ : nourseothricin resistance marker, ${ }^{p}$ : promoter, ${ }^{t}:$ terminator, MT: Master thesis. 


\subsubsection{Plasmid and strain construction of $V$. longisporum strains}

\section{Plasmid and strain construction of VI43 LSI deletion}

For construction of the LSI ( $\Delta$ v/43ass51scaf493:522667-534160) deletion cassette, the 2005 bp upstream flanking region was amplified with primers JST1/JST2 and a 1023 bp downstream flanking region with primers JST3/JST4 from fungal wild type DNA. The $2194 \mathrm{bp} N A T^{R}$ marker cassette was amplified from pME4815 with primers ML8/ML9. The fragments were ligated to the backbone pME4564 which was PCR amplified using primers ML1/ML2, resulting in plasmid pME4816 used for wild type transformation. Correct replacement of the $L S I$ region by the $N A T^{R}$ marker cassette was confirmed by Southern hybridization using the $5^{\prime}$ flanking region amplified with JST1/JST2 as probe and restriction with Sspl (Figure S1). VI43 $\Delta L S I$ deletion transformants were conserved as VGB201 and VGB233.

\section{Plasmid and strain construction of VI43 LSII deletion}

For construction of the LSII ( $\Delta$ v/43ass51scaf493:534161-542607) deletion cassette, the $1041 \mathrm{bp}$ upstream flanking region was amplified with primers JST5/JST6 and the 2016 bp downstream flanking region with primers JST7/JST8 from fungal wild type DNA. The $N A T^{R}$ marker cassette and the used backbone were amplified as described for the VI43 LSI deletion strain and ligated, resulting in plasmid pME4817 used for wild type transformation. Correct replacement of the $L S / /$ region by the $N A T^{R}$ marker cassette was confirmed by Southern hybridization using the 3'-flanking region amplified with JST7/JST8 as probe and restriction with Nhel (Figure S1). VI43 $\Delta$ LSII deletion transformants were conserved as VGB193 and VGB214.

\section{Plasmid and strain construction of VI43 LS deletion}

For construction of the LS ( $\Delta$ v/43ass51scaf493:522667-542607) deletion cassette, the $2005 \mathrm{bp}$ upstream flanking region of the VI43LS20kb region was amplified with primers JST1/JST2 and the 2016 bp downstream flanking region was amplified with primers JST7/JST8 from fungal wild type DNA. The $H Y G^{R}$ marker cassette was amplified from pPK2 with primers RO3/RO4. The fragments were ligated to the plasmid pME4564, which was PCR-amplified using the primers ML1/ML2, resulting in plasmid pME4818 used for VI43 $\triangle L S I$ transformation. Correct replacement of the previously introduced $N A T^{R}$ marker cassette and the $L S I /$ region by the $H Y G^{R}$ marker cassette was confirmed by Southern hybridization using the $5^{\prime}$ - and $3^{\prime}$-flanking region as probes amplified with JST1/JST2 (5') and JST7/JST8 (3') and restriction with Sspl (5') and Nhel (3'; Figure S1). The resulting $L S$ deletion transformant was conserved as VGB232. 


\subsubsection{Plasmid and strain construction of $V$. dahliae strains}

\section{Plasmid and strain construction of the $V$. dahliae $L S$ deletion}

For construction of the V. dahliae LS deletion cassette, $1997 \mathrm{bp}$ up- and $2106 \mathrm{bp}$ downstream of the VI43LS20kb homologous region were amplified from $V$. dahliae JR2 genomic DNA using primers JST210/JST2 (5') and JST7/JST8 (3'). The nourseothricin resistance marker cassette was amplified from pME4815 with primers ML8/ML9 and ligated into pME4564 cut with the restriction enzymes EcoRV and Stul. The resulting plasmid pME4829 was used for $V$. dahliae JR2 transformation. Correct replacement of the VI43LS20kb homologous region by the $N A T^{R}$ marker cassette was confirmed by Southern hybridization using the flanking regions amplified with JST210/JST2 (5') as probes and JST7/JST8 (3') and restriction with Mlul (5', not shown) or Bgll (3'; Figure S3). The resulting $V$. dahliae JR2 $L S$ deletion transformants were conserved as VGB383 and VGB393.

\section{Plasmid and strain construction of the HAM5 single and double deletion with VMK1}

For construction of the HAM5 (VDAG_JR2_Chr4g07170a) deletion cassette, the $1500 \mathrm{bp}$ flanking region $333 \mathrm{bp}$ upstream of the open reading frame (ORF) was amplified with primers JST110/JST111 and the 1000 bp downstream flanking region was amplified with primers JST112/JST113 from wild type fungal DNA. The $2194 \mathrm{bp} N A T^{R}$ marker cassette was amplified with ML8/ML9 from pME4815. The fragments were ligated into the 6728 bp backbone pME4564 amplified with primers ML1/ML2. The resulting plasmid pME4820 was used for wild type and $\triangle V M K 1$ (VGB335) transformation. 5'-and 3'flanking regions of HAM5 were amplified using primers JST110/JST111 (5') and JST112/JST113 $\left(3^{\prime}\right)$, respectively and labeled as probes for Southern hybridization. Restriction enzymes $\mathrm{Xhol}\left(5^{\prime}\right.$, not shown) and Sacl (3') were used to cut genomic DNA (Figure S5). The resulting HAM5 single deletion transformants were conserved as VGB279 and VGB280, the HAM5 and VMK1 double deletion transformant was conserved as VGB417.

\section{Plasmid and strain construction of the ectopic HAM5 complementation}

For construction of the ectopic HAM5 complementation cassette, a 7265 bp sequence including 1359 bp 5'-flanking region, 4906 bp HAM5 ORF, and 1000 bp 3'-flanking region was amplified using JST245/JST246 from fungal genomic DNA. The PCR product was ligated into pPK2 harboring the $H Y G^{R}$ marker cassette cut with the restriction enzyme EcoRV. The resulting plasmid was named pME4828 and used for $\triangle H A M 5$ transformation. Flanking regions of HAM5 were amplified using primers JST110/JST111 $\left(5^{\prime}\right)$ and JST112/JST113 (3') and labeled as probes for Southern hybridization. 
Restriction enzymes Pvull (5', not shown) and Sacl (3') were used to cut genomic DNA (Figure S5). The resulting HAM5-C complementation transformant was conserved as VGB415.

\section{Plasmid and strain construction of the VMK1 deletion}

For construction of the VMK1 (VDAG_JR2_Chr2g01260a) deletion cassette, the $898 \mathrm{bp}$ $3^{\prime}$-flanking region was amplified with primers JS-V21/JS-V22 from fungal wild type DNA and ligated to the 10739 bp pPK2 backbone with $H Y G^{R}$ marker cassette amplified with JS-V23/JS-V24, resulting in pME4824. The 1472 bp 5'-flanking region was amplified with primers JST77a/JST76b from fungal wild type DNA and cloned into pME4824, amplified with primers ML1/JS-V23, resulting in pME4825 used for wild type transformation. Flanking regions of VMK1 were amplified using primers JST77a/JST76b (5') and JS-V21/JS-V22 (3') and labeled as probes for Southern hybridization. Restriction enzymes Xhol (5', not shown) or Bgll (3') were used to cut genomic DNA (Figure S6). The resulting VMK1 deletion transformants were conserved as VGB335 and VGB336.

\section{Plasmid and strain construction of the ectopic VMK1 complementation}

For construction of the ectopic VMK1 complementation cassette, a 3661 bp sequence including 1473 bp 5'-flanking region, 1260 bp VMK1 ORF, and 928 bp 3'-flanking region, was amplified from fungal wild type DNA using JST243/JST244. The PCR product was ligated to pME4815 harboring a $N A T^{R}$ marker cassette cut with the restriction enzyme EcoRV, resulting in the plasmid pME4827 used for $\triangle V M K 1$ transformation. Flanking regions of VMK1 were amplified using primers JST77a/JST76b (5') and JS-V21/JS-V22 (3') and labeled as probes for Southern hybridization. The restriction enzymes Vspl (5', not shown) or Bgll (3') were used to cut genomic DNA (Figure S6). The resulting VMK1-C complementation transformant was conserved as VGB413.

\section{Plasmid and strain construction of the MEK2 single and double deletion with HAM5}

For construction of the MEK2 (VDAG_JR2_Chr1g13070a) deletion cassette the $1500 \mathrm{bp}$ $5^{\prime}$-flanking region was amplified with primers JS-V5/JS-V6 from fungal wild type DNA and ligated to the 10676 bp pPK2 backbone with $H Y G^{R}$ marker cassette amplified with JS-V7/JS-V8, resulting in pME4821.

The 1500 bp 3 'flanking region 385 bp downstream of the ORF was amplified with primers JS-V9/JS-V10 from fungal wild type DNA and ligated to pME4821 amplified with primers JS-V11/JS-V12, resulting in pME4822 used for wild type and $\triangle H A M 5$ (VGB279) transformation. Flanking regions of MEK2 were amplified using primers JS-V5/JS-V6 (5') and JS-V9/JS-V10 (3') and labeled as probes for Southern hybridization. The restriction enzymes Hindll (5', not shown) or Nrul (3') were used to cut genomic DNA (Figure S7). 
The resulting MEK2 single deletion transformants were conserved as VGB337 and VGB338, the MEK2 and HAM5 double deletion transformant was conserved as VGB346.

\section{Plasmid and strain construction of the ectopic MEK2 complementation}

For construction of the ectopic MEK2 complementation cassette, a 5176 bp sequence

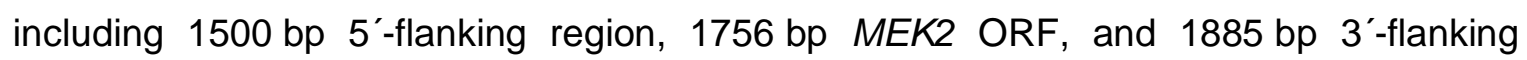
region was amplified from fungal wild type DNA using JST212/JST213. The PCR product was ligated to pME4815 harboring a $N A T^{R}$ marker cassette cut with the restriction enzyme EcoRV, resulting in the plasmid pME4826 used for $\triangle M E K 2$ transformation. Flanking regions of MEK2 were amplified using primers JS-V5/JS-V6 (5') and JSV9/JS-V10 (3') and labeled as probes for Southern hybridization. The restriction enzymes Hindlll (5', not shown) or Nrul (3'; Figure S7) were used to cut genomic DNA. The resulting MEK2-C complementation transformant was conserved as VGB389.

\section{Plasmid and strain construction of the HAC1 deletion}

For construction of the HAC1 (VDAG_JR2_Chr2g09780a) deletion cassette, a $1500 \mathrm{bp}$ flanking region 218 bp upstream from the ORF was amplified with JST186/JST187 and a 1445 bp 3 '-flanking region was amplified using JST188/JST189 from fungal wild type DNA. The $3942 \mathrm{bp} H Y G^{R}$ marker cassette was amplified from pPK2 with primers ML8/RO3. The fragments were ligated to pME4564 cut with restriction enzymes EcoRV and Stul, resulting in pME4830 used for wild type transformation. 1500 bp up- and $300 \mathrm{bp}$ downstream flanking regions of HAC1 were amplified using primers JST186/JST187 (5') and JST272/JST273 (3') and labeled as probes for Southern hybridization. Restriction enzyme Sall (5', not shown; 3'(Figure S8)) was used to cut genomic DNA. The resulting HAC1 deletion transformants were conserved as VGB371 and VGB372.

\section{Plasmid and strain construction of the ectopic HAC1 complementation}

For construction of the ectopic HAC1 complementation cassette, a 3636 bp insert containing 1702 bp 5'-flanking, 1634 bp HAC1 gene, and 300 bp 3'-flanking region was amplified with primers JST216/JST211 and cloned into pME4815 cut with the restriction enzyme EcoRV, resulting in plasmid pME4831 used for transformation of the $\triangle H A C 1$ strain. For Southern hybridization (Figure S8) the same restriction enzymes and probes were used as for verification of the HAC1 deletion strain. The resulting HAC1-C complementation transformant was conserved as VGB382.

\section{Plasmid and strain construction of the ectopic HAC1"-HA complementation}

For construction of a $H A C 1^{u}-H A$ complementation construct the uninduced mRNA splice variant of HAC1 without stop codon was amplified with primers JST171/JST172 from 
complementary DNA (cDNA) isolated from wild type cultures incubated in SXM for four days. The C-terminal $H A$-tag was fused to $H A C 1^{u}$ via amplification with a 48 bp $H A$ sequence as overhang using JST171/JST267. HAC14-HA was ligated to the cloning vector pJet1.2, resulting in pME4832.

The 1400 bp 5'-flanking region was amplified with primers JST269/JST270 and a 300 bp 3 '-flanking region was amplified with primers JST272/JST273 from fungal wild type DNA. The 1626 bp HAC1 ${ }^{u}-H A$ sequence was amplified from pME4832 with JST171/JST268. The fragments were ligated to pME4815 cut with the restriction enzyme EcoRV, resulting in pME4834 used for transformation of the $\triangle H A C 1$ strain. For Southern hybridization the same probes were used as for verification of the HAC1 deletion strain. The restriction enzymes Pvull (5', not shown) and Sall (3'; Figure S8) were used to cut genomic DNA. The resulting HAC1 ${ }^{u}-H A$ transformants were conserved as VGB439 and VGB440.

\section{Plasmid and strain construction of the ectopic HAC1-HA complementation}

For construction of a $H A C 1^{i}-H A$ complementation construct, the induced splice variant of HAC1 without stop codon was amplified from cDNA isolated from wild type cultures incubated in SXM for four days with subsequent supplementation with $3 \mathrm{mM}$ dithiothreitol (DTT) for three hours using primers JST171/JST174. The C-terminal HA-tag was fused to $H A C 1^{i}$ via amplification with a $48 \mathrm{bp} H A$ sequence as overhang using JST171/JST266. $H A C 1^{i}-H A$ was ligated to the cloning vector pJet1.2, resulting in pME4833 used for transformation of the $\triangle H A C 1$ strain. The 1400 bp $5^{\prime}$-flanking region was amplified with primers JST269/JST270 and a 607 bp 3'-flanking region was amplified with primers JST271/JST272 from fungal wild type DNA. The $1299 \mathrm{bp} H A C 1^{i}-H A$ sequence was amplified from pME4833 with JST171/JST268. The fragments were ligated to pME4815 cut with the restriction enzyme EcoRV, resulting in pME4835 used for transformation of the $\triangle H A C 1$ strain. For Southern hybridization the same probes were used as for verification of the HAC1 deletion strain. The restriction enzymes Pvull (5', not shown) and Sall (3'; Figure S8) were used to cut genomic DNA. The resulting HAC1'-HA transformants were conserved as VGB437 and VGB438.

\section{Plasmid and strain construction of the ectopic GFP overexpression strains JR2 OE-GFP and $\triangle H A C 1 O E-G F P$}

For construction of the GFP overexpression vector with $N A T^{R}$ resistance marker cassette, a 2378 bp fragment containing GFP under control of the gpdA promoter and trpC terminator was amplified from pGreen2 with primers JST184/JST185 and ligated to pME4815 amplified with primers ML1/ML9, resulting in pME4819 used for wild type and $\triangle H A C 1$ strain transformation. 
The resulting JR2 OE-GFP transformant was confirmed by fluorescence microscopy and phenotypic comparison to wild type and conserved as VGB392.

The resulting $\triangle H A C 1$ OE-GFP transformant was confirmed by fluorescence microscopy, phenotypic comparison to the $\triangle H A C 1$ strain and Southern hybridization. The $300 \mathrm{bp}$ downstream flanking region of HAC1 was amplified using primers JST272/JST273 and labeled as probe for Southern hybridization. The restriction enzyme Sall (3'; Figure S8) was used to cut genomic DNA. The resulting $\triangle H A C 1$ OE-GFP transformant was conserved as VGB380.

\section{Plasmid and strain construction of the ODE1 deletion}

For construction of the ODE1 (VDAG_JR2_Chr1g29610a) deletion cassette, the $1000 \mathrm{bp}$ 5 '- and 1522 bp $3^{\prime}$-flanking regions were amplified from fungal wild type DNA with primers JST127/JST128 (5') or JST129/JST130 (3'). The 2194 bp NAT marker cassette was amplified with ML8/ML9 from pME4815 and ligated to the $6728 \mathrm{bp}$ pME4564 backbone amplified with JST137/JST138, resulting in pME4836 used for wild type transformation. Amplified flanking regions of ODE1 were labeled as probes for Southern hybridization. The restriction enzymes Bgl (5', not shown) or Scal (3', Figure S9) were used to cut genomic DNA. The $\triangle O D E 1$ strains were conserved as VGB331 and VGB332.

\section{Plasmid and strain construction of endogenous GFP-tagged ODE1 complementation}

For construction of the ODE1 complementation cassettes coding for either $\mathrm{N}$ - or $\mathrm{C}$ terminal GFP-tagged Ode1, the 1522 bp 3'-flanking region was amplified from fungal wild type DNA with primers JST129/JST130 and ligated to the 10744 bp pPK2 backbone amplified with JST138/JST177, resulting in plasmid pME4837.

For construction of the endogenous ODE1-GFP complementation cassette, a $2501 \mathrm{bp}$ PCR product containing the $1000 \mathrm{bp} 5^{\prime}$-flanking region and $1501 \mathrm{bp} O D E 1$ gene without stop codon was amplified from fungal wild type DNA with JST179/JST180. The sequence of 735 bp coding for C-terminal GFP with a 5 aa linker was amplified with primers SAB16/JST178 from pGreen2. Both inserts were ligated to pME4837 cut with the restriction enzyme EcoRV, resulting in plasmid pME4838 used for transformation of the $\triangle O D E 1$ strain.

For construction of the endogenous GFP-ODE1 complementation cassette the $1000 \mathrm{bp}$ $5^{\prime}$-flanking region was amplified with JST180/JST181 and the 1504 bp ODE1 gene was amplified with JST182/JST183 from fungal wild type DNA. The 732 bp sequence coding for N-terminal GFP and a 5 aa linker was amplified with primers JST98/ZQY11 from 
pGreen2. Inserts were ligated to pME4837 cut with the restriction enzyme EcoRV, resulting in plasmid pME4839 used for transformation of the $\triangle O D E 1$ strain.

Amplified flanking regions of $O D E 1$ were labeled as probes for Southern hybridization as described in for the plasmid and strain construction of the $O D E 1$ deletion. The restriction enzymes Avall (5', not shown) or Scal (3', Figure S9) were used to cut genomic DNA. The expression of the fusion protein was confirmed by fluorescence microscopy and immunoblotting with a GFP-protein binding antibody. The strains were conserved as VGB358 and VGB359 (ODE1-GFP) and VGB360 and VGB361 (GFP-ODE1).

\subsection{Microbiological methods}

\subsubsection{Transformation of Escherichia coli}

Chemically competent $E$. coli DH5a cells were transformed by the heat shock method in a modified protocol according to Hanahan et al., 1991. $200 \mu$ competent E. coli cells were thawed on ice, mixed with the ligation mixture or plasmid solution, and incubated on ice for $30 \mathrm{~min}$. A heat shock at $42^{\circ} \mathrm{C}$ was induced for one minute. Afterwards, the E. coli cells were chilled on ice for two minutes. $800 \mu \mathrm{l} \mathrm{SOC}$ medium was added and the cells were incubated for $50 \mathrm{~min}$ at $37^{\circ} \mathrm{C}$ under constant agitation. Positive transformants were selected on solid LB plates, supplemented with $100 \mu \mathrm{g} / \mathrm{ml}$ ampicillin or $100 \mu \mathrm{g} / \mathrm{ml}$ kanamycin. The plates were incubated at $37^{\circ} \mathrm{C}$ overnight and the transformants were tested by colony PCR for plasmid uptake.

\subsubsection{Transformation of Agrobacterium tumefaciens}

A. tumefaciens cells were transformed with plasmid DNA as described in Bui 2017 (Dissertation, University of Goettingen). The heat shock was performed for five minutes at $37^{\circ} \mathrm{C}$.

\subsubsection{Transformation of Verticillia}

Verticillia were transformed by $A$. tumefaciens-mediated transformation as described in Mullins et al., 2001. A. tumefaciens cultures were inoculated in $5 \mathrm{ml}$ liquid LB medium supplemented with $100 \mathrm{\mu g} / \mathrm{ml}$ kanamycin and incubated overnight at $25^{\circ} \mathrm{C}$ on a rotary shaker. $20 \mathrm{ml}$ induction medium (IM) [1x MM salts $\left(2.5 \mathrm{x}\right.$ stock: $3.625 \mathrm{~g} / \mathrm{K} \mathrm{KH}_{2} \mathrm{PO}_{4}$, $5.125 \mathrm{~g} / / \mathrm{K}_{2} \mathrm{HPO}_{4}, 0.375 \mathrm{~g} / \mathrm{l} \mathrm{NaCl}, 1.25 \mathrm{~g} / \mathrm{l} \mathrm{MgSO}{ }_{4} \cdot 7 x \quad \mathrm{H}_{2} \mathrm{O}, 0.165 \mathrm{~g} / \mathrm{l} \mathrm{CaCl} \cdot 2 \mathrm{C} \mathrm{H}_{2} \mathrm{O}$, $\left.\left.6.2 \mathrm{mg} / \mathrm{FeSO}_{4} \cdot 7 x \mathrm{H}_{2} \mathrm{O}, 1.25 \mathrm{~g} / \mathrm{l}\left(\mathrm{NH}_{4}\right)_{2} \mathrm{SO}_{4}\right)\right], 400 \mathrm{ml} / \mathrm{l}, 10 \mathrm{mM}$ glucose, $0.5 \%$ glycerol, $40 \mathrm{mM}$ MES monohydrate (Carl Roth GmbH\&Co.KG) supplemented with $200 \mu \mathrm{M}$ acetosyringone (AS) were inoculated with $0.5 \mathrm{ml}$ to $1 \mathrm{ml}$ of the overnight culture and incubated for four to five hours at $28^{\circ} \mathrm{C}$ under constant agitation in a water bath. Equal amounts of $A$.tumefaciens liquid culture and Verticillium spore solutions with 
$1 \times 10^{6}$ spores $/ \mathrm{ml}$ were mixed and $200 \mu \mathrm{l} /$ plate of the mixture were distributed on IM solid medium (same as liquid IM except that it contains $5 \mathrm{mM}$ glucose and $1.5 \%$ agar, supplementation of sterile MES and AS after autoclaving) covered with a sterile nitrocellulose filter ( $\varnothing=85 \mathrm{~mm}$, Sartorius, Goettingen, Germany). After three days of incubation at $25^{\circ} \mathrm{C}$, filters were transferred to solid PDM supplemented with respective antimycotica hygromycin B $(50 \mu \mathrm{g} / \mathrm{ml})$ and/or nourseothricin $(72 \mu \mathrm{g} / \mathrm{ml})$ and cefotaxime $(300 \mu \mathrm{g} / \mathrm{ml})$. Filters were removed after six to ten days and transformants were separated twice on solid PDM selection plates.

\subsubsection{Phenotypic analysis and stress tests}

For characterization of the ex planta phenotypes, Verticillium strains were analyzed by spot inoculation of 50000 freshly harvested spores on different solid media, including SXM, PDM, Malt extract agar [30\% malt extract (Carl Roth GmbH\&Co.KG), 5\% BD Bacto Peptone (BD Biosciences), 20\% agar, pH 7.6], CDM [liquid CDM with 2\% agar], CDM with alternative carbon sources [ $3 \%$ cellulose (Carboxymethylcellulose sodium salt, Sigma-Aldrich Chemie $\mathrm{GmbH}$ ), 3\% galactose, or $3 \%$ glucose] and incubated at $25{ }^{\circ} \mathrm{C}$ for 9-14 days. For induction of oxidative stress, CDM was supplemented with $0.00075 \%$ $\mathrm{H}_{2} \mathrm{O}_{2}$ or $0.004 \%$ SDS. CDM supplemented with $0.5 \mathrm{M} \mathrm{NaCl}$ or $0.8 \mathrm{M}$ Sorbitol was used for induction of osmotic stress. ER stress induction was achieved by CDM supplementation with $1 \mu \mathrm{g} / \mathrm{ml}$ tunicamycin. CDM supplementation with linoleic acid (LA, $0.125 \mathrm{mg} / \mathrm{ml}$ ) was used for induction of oxidative stress and for complementation of partial auxotrophy in ODE1 deletion strains.

\subsubsection{Growth quantification}

For growth quantification, 50000 freshly harvested spores were spot inoculated on three $30 \mathrm{ml}$ plates per medium and transformant. Growth was quantified by measurement of two perpendicular diameters per colony at indicated growth conditions. The mean value of three colonies per transformant was considered as one biological replicate $(n=1)$. Significances were calculated using the one-way Anova and Student's t-test from Simple Interactive Statistical Analysis (SISA) online tool (http://www.quantitativeskills.com/sisa/ tableprocs/meanst.htm).

\subsubsection{Microsclerotia observation and quantification of melanization}

Microsclerotia formation was visualized by microscopy of colony cross sections with a binocular microscope (Olympus Deutschland $\mathrm{GmbH}$ ) and microscopy of material scraped off with a scalpel from the colony center using an Axiolab light microscope (Zeiss). Calibration of pictures for magnification was done using the cellSens Dimension version 1.4 software (Olympus $\mathrm{GmbH})$. 
For quantification of melanization, 50000 freshly harvested spores were spot inoculated on three $30 \mathrm{ml}$ CDM with cellulose medium plates per transformant. Pictures from colonies were taken nine days after spot inoculation from the top view after removal of aerial mycelium and the melanized area was measured from eight bit greyscale pictures using ImageJ software (Rasband, 1997). The means of the brightness factor were determined, set relative to wild type, and inverted. The mean value of three colonies per transformant was considered as one biological replicate $(n=1)$. Significances were calculated using the one-way Anova and Student's t-test from SISA online tool.

\subsubsection{Conidiospore quantification}

For spore quantification, freshly harvested conidia were inoculated in liquid SXM to a concentration of 4000 conidiospores $/ \mathrm{ml}$ in triplicates and incubated for five days under constant agitation at $135 \mathrm{rpm}$ in three independent experiments. Spores were harvested through Miracloth (Calbiochem Merck) and diluted in equal volumes of sterile water. Conidiospore concentrations were measured three times per spore solution using the Coulter Z2 Particle Count and Size Analyzer (Beckman Coulter, Krefeld, Germany) and the appropriate Coulter Isoton II Diluent. The mean number of spores from a single transformant per experiment represents $n=1$. Numbers of spores were normalized to the mean value of three independent experiments determined for wild type. The values determined for two independent transformants from one genotype were summed up in one bar. Error bars indicate the standard deviations. Significances were calculated using the one-way Anova and Student's t-test from SISA online tool.

\subsubsection{Localization assay}

Localization of Ode1 $\mathrm{N}$ - or C-terminally tagged to GFP was examined by fluorescence microscopy. $300 \mu \mathrm{l}$ liquid PDM was inoculated with 50 000-100 000 freshly harvested spores per well in an 8 well microscopy chamber (Ibidi) and incubated at $25^{\circ} \mathrm{C}$ for 12 20 hours. Hyphal morphology and subcellular localization were observed with a PlanNeofluar 100x/1.4 oil objective (Zeiss) with 300 ms exposure time for GFP signals and $800 \mathrm{~ms}$ exposure time for RFP signals. Vacuoles were visualized by fluorescence microscopy after staining with 0.3 $\mu$ FM4-64 Dye (Thermo Fisher Scientific) for one hour in the dark.

\subsubsection{Plant infection assays}

\subsubsection{Brassica napus infection assay with $V$. longisporum}

$B$. napus plants were used as host for infection assays with $V$. longisporum strains. Per experiment 15 seven-day-old seedlings of oilseed rape were tested by root dipping into a 
solution of $1.5 \times 10^{6}$ conidiospores $/ \mathrm{ml}$ and the mock control plants were treated with sterile demineralized water for 40 min under constant agitation at $\sim 30 \mathrm{rpm}$. The seedlings were transferred to $70 \times 70 \times 80 \mathrm{~mm}$ planting pots (Soparco $\mathrm{GmbH}$ ) containing a sand:soil (1:1) mixture (Dorsilit, Archut GmbH\&Co.KG). The soil was inoculated with either 2.25 $\times 10^{6}$ spores or $1.5 \mathrm{ml}$ sterile water for mock plants per pot. The plants were allowed to grow in a climate chamber BrightBoy GroBank (CLF PlantClimatics) at long day conditions with 16 hours of light (fluorescence: 60, GroLEDs: 100, illumination: $95 \mu \mathrm{mol}$ ) at $25^{\circ} \mathrm{C}$ and eight hours of darkness at $22^{\circ} \mathrm{C}$. The disease symptoms were scored after 35 days according to the disease rating criteria. The numbers of tested plants, transformants, and independent experiments are indicated.

\subsubsection{Solanum lycopersicum infection assay with $V$. dahliae}

S. lycopersicum (moneymaker, B17422) plants were used as host for plant infection assays with $V$. dahliae strains. Per experiment 15 ten-day-old seedlings of tomato were tested by root dipping into a solution of $1 \times 10^{7}$ conidiospores $/ \mathrm{ml}$ or $3 \mathrm{ml}$ sterile water for the mock control plants for 40 min under constant agitation at $\sim 30 \mathrm{rpm}$. The seedlings were transferred to $70 \times 70 \times 80 \mathrm{~mm}$ planting pots (Soparco) containing a sand:soil (1:1) mixture (Dorsilit, Archut). The soil was inoculated with $3 \times 10^{7} \mathrm{~V}$. dahliae spores or sterile water for mock plants per pot. The plants were allowed to grow in a climate chamber at long day conditions with 16 hours of light (fluorescence: 60, GroLEDs: 100, illumination: $95 \mu \mathrm{mol})$ at $25^{\circ} \mathrm{C}$ and eight hours of darkness at $22^{\circ} \mathrm{C}$. The disease symptoms were scored after 21 days according to the disease rating criteria. The numbers of tested plants, transformants and independent experiments are indicated.

\subsubsection{Disease rating criteria}

Disease symptoms induced in $B$. napus and $S$. lycopersicum infection assays with $V$. longisporum and $V$. dahliae were scored according to the following disease rating criteria: The fresh weight excluding the roots, the length of the longest leaf, and the height of the vegetation point per plant were determined and transformed into a score ranging from 1 to 5 relative to the values determined for the uninfected mock plants (rapeseed plants: $100-80 \%($ mock $)=1,79-60 \%($ mock $)=2,59-40 \%($ mock $)=3,<40 \%$ $($ mock $)=4$, dead plant $=5$; tomato plants: $100-70 \%($ mock $)=1,69-60 \%($ mock $)=2,59-$ $40 \%($ mock $)=3,<40 \%($ mock $)=4$, dead plant $=5)$. Furthermore, a leave symptom index per plant was calculated (leave symptoms score $=[$ (number of healthy leaves $x 1$ ) $+($ number of leaves with weak symptoms $\times 2)+($ number of leaves with strong symptoms $x 3)+($ number of dead leaves $x 4$ )] / total number of leaves) for rapeseed plants. The disease score per plant was calculated by the mean of the scores for each parameter 
[height, length of the longest leaf, weight, leave symptom index (for rapeseed plants only)] ranging from 1 to 5 ( 1 = healthy, 2 = weak symptoms, $3=$ strong symptoms, $4=$ very strong symptoms, $5=$ dead plant). The number of plants categorized into the disease scores relative to the total number of tested plants from all experiments was visualized in a stack diagram. One bar represents all tested plants from independent experiments inoculated with a single or two independent transformants of the same genotype as indicated.

For tomato plants discoloration of the hypocotyl was examined by observation of cross sections with a binocular microscope (Olympus Deutschland $\mathrm{GmbH}$ ).

For rapeseed plants inoculated with $V$. longisporum strains, statistical analysis was performed to calculate significant differences regarding the number of dead plants. Oneway Anova and Student's t-test from SISA online tool were used for calculation of p-values.

\subsubsection{Stem assay}

To test for fungal outgrowth from plants inoculated with fungal spores or water control, stems were harvested after 21 days for tomato plants or 35 days for rapeseed plants. The stems were surface sterilized by washing with $70 \%$ ethanol for eight minutes, followed by $6 \%$ sodium hypochlorite solution for eight minutes and subsequent washing with distilled sterile water, twice. The stem ends were removed and slices of the middle part were placed on PDM plates supplemented with chloramphenicol $(100 \mu \mathrm{g} / \mathrm{ml})$. Plates were incubated at $25^{\circ} \mathrm{C}$ for seven days and fungal growth was documented.

Fungal outgrowth from $V$. dahliae $\triangle V M K 1, \triangle M E K 2, \triangle H A M 5 \triangle V M K 1$ and $\triangle H A M 5 \triangle M E K 2$ strains was confirmed by control PCR reactions using genomic DNA and primers A0144/JST288 ( $\triangle$ VMK1, $\triangle H A M 5 \triangle V M K 1)$ resulting in a $3044 \mathrm{bp}$ PCR product and AO144/JST289 ( $\triangle M E K 2, \triangle H A M 5 \triangle M E K 2)$ resulting in a 3013 bp PCR product. AO144 targets the $g p d A$ promoter in the hygromycin resistance marker cassette, JST288 binds $156 \mathrm{bp}$ upstream of the $1473 \mathrm{bp} 5^{\prime}$-flanking region of VMK1 used for homologous recombination, and JST289 binds $89 \mathrm{bp}$ upstream of the $1500 \mathrm{bp} 5^{\prime}$-flanking region of MEK2 used for homologous recombination.

\subsubsection{Arabidopsis thaliana root colonization assay}

Analysis of root colonization capabilities of Verticillium strains was performed with A. thaliana (Col-0) plants. The root colonization assay was modified from Tran et al. 2014. Seeds were surface sterilized for five minutes in a solution containing $70 \% \mathrm{EtOH}$ and $0.05 \%$ Tween 80 and dried at $35{ }^{\circ} \mathrm{C}$ for $20 \mathrm{~min}$. The sterilized seeds were placed on Murashige and Skoog (MS; Murashige \& Skoog, 1962) plates [0.22\% MS + vitamin 
(Duchefa, Haarlem, Netherlands), 0.05\% MES monohydrate (Carl Roth GmbH\&Co.KG), $1 \%$ sucrose and $1.5 \%$ plant agar (Duchefa), pH 5.7], incubated at $4{ }^{\circ} \mathrm{C}$ overnight and transferred to the climate chamber for incubation at long day conditions with 16 hours of light (fluorescence: 60 , GroLEDs: 100 , illumination: $95 \mu \mathrm{mol}$ ) at $25^{\circ} \mathrm{C}$ and eight hours of darkness at $22{ }^{\circ} \mathrm{C}$. After 21 days, plants were transferred to $1 \%$ water agarose. One day later, roots were inoculated in fresh conidial suspensions $\left(1 \times 10^{5}\right.$ spores $\left./ \mathrm{ml}\right)$ for $35 \mathrm{~min}$ and transferred back to $1 \%$ water agarose. Two thirds of the plate were covered with aluminum foil to shade the roots. Afterwards, plants were incubated in a plant chamber at long day conditions for the indicated time.

For microscopy, roots were cut and incubated for five minutes in the dark in a staining solution $[0.0025 \%$ propidium iodide and $0.005 \%$ silwet]. The roots were transferred to an object slide with $150 \mu \mathrm{l} \mathrm{H}_{2} \mathrm{O}$ and $50 \mu \mathrm{l}$ staining solution. Cover slips were fixed with nail polish. Fungal root colonization was examined by fluorescence microscopy. Overview pictures [20x objective (Zeiss)] as well as close up pictures [63x objective (Zeiss)] were taken with $300 \mathrm{~ms}$ exposure time for GFP signals and $800 \mathrm{~ms}$ exposure time for RFP signals. Volume view pictures shown are maximum intensity projections of a Z-stack of microscope images. Per experiment, fungal strain, and time point root colonization was analyzed for two to three independent plants.

\subsection{Nucleic acid methods}

\subsubsection{Nucleic acid purification}

\section{Purification of PCR products and linearized DNA fragments}

PCR products used for cloning or as probes for Southern hybridization analysis, as well as fragments cut with restriction enzymes, were separated from side products on an agarose gel by gel electrophoresis, excised from the gel, and purified using the NucleoSpin Gel and PCR Clean-up Kit (Macherey-Nagel) according to the manufacturer's instructions.

\section{Plasmid purification from $E$. coli}

For isolation of a plasmid of interest, $E$. coli strains were inoculated in $5 \mathrm{ml}$ LB medium supplemented with the corresponding antibiotic and incubated at $37^{\circ} \mathrm{C}$ on a rotary shaker overnight. Plasmid DNA was purified with the NucleoSpin Plasmid Kit from Macherey-Nagel according to the manufacturer's protocol.

\section{Genomic DNA purification from Verticillium}

The extraction method was modified from Kolar et al., 1988. For the isolation of genomic DNA of Verticillium, mycelium was harvested from liquid PDM four to seven days post 
inoculation by filtration through Miracloth filters (Calbiochem Merck). The mycelium was rinsed with $0.96 \% \mathrm{NaCl}$ solution, dried, frozen, and ground to fine powder in liquid nitrogen. The powder was mixed with $800 \mu$ l of lysis buffer [50 mM tris pH 7.5, $50 \mathrm{mM}$ EDTA pH 8, 3\% SDS and 1\% B-mercaptoethanol]. The mixture was incubated at $65{ }^{\circ} \mathrm{C}$ for one hour and subsequently mixed with $800 \mu$ phenol. Phases were separated by centrifugation for $20 \mathrm{~min}$ at $13000 \mathrm{rpm}$ and $4{ }^{\circ} \mathrm{C}$. The upper phase was transferred into a new tube, mixed with $500 \mu \mathrm{l}$ chloroform for denaturation of proteins and centrifuged for ten minutes at $13000 \mathrm{rpm}$ and $4{ }^{\circ} \mathrm{C}$. The upper phase was transferred into a new tube, mixed with $400 \mu \mathrm{l}$ isopropanol and centrifuged for two minutes at $13000 \mathrm{rpm}$ for precipitation of genomic DNA. The precipitated genomic DNA was desalted by washing with $300 \mu \mathrm{l} 70 \%$ ethanol and centrifugation for one minute at $13000 \mathrm{rpm}$. Ethanol was removed and the precipitated genomic DNA was dried with open lid at $65^{\circ} \mathrm{C}$ for $20 \mathrm{~min}$. The sediment was resuspended in 50-100 $\mu$ deionized $\mathrm{H}_{2} \mathrm{O}$ and RNA was cut by RNase A $(200 \mu \mathrm{g} / \mathrm{ml})$ at $65^{\circ} \mathrm{C}$ for $30 \mathrm{~min}$. Quality and concentration of genomic DNA were tested by agarose gel electrophoresis.

\section{RNA purification from Verticillium}

RNA was purified from fungal mycelium harvested at indicated time points from indicated growth conditions using the Direct-zol RNA MiniPrep Kit from Zymo Research according to the manufacturer's instructions. Mycelium was rinsed with $0.96 \% \mathrm{NaCl}$ solution, dried, frozen, and ground to fine powder in liquid nitrogen. Approximately $1 \mathrm{ml}$ of the powder was transferred to precooled $15 \mathrm{ml}$ Falcon tubes, mixed with $1 \mathrm{ml}$ TRIzol (Ambion and life technologies, Carlsbad, CA, USA), and frozen in liquid nitrogen.

DNase I was used to cut remaining DNA on columns according to the manufacturer's protocol. Purified RNA was eluted in prewarmed DNase/RNase free water. Quality and concentration of RNA were defined by determination of the absorption rations at $260 \mathrm{~nm} /$ $280 \mathrm{~nm}(\sim 1.8)$ and $260 \mathrm{~nm} / 230 \mathrm{~nm}$ ( 2.0-2.2) for a 1:10 dilution using the Nanodrop ND1000 spectrophotometer (PeqLab Biotechnology $\mathrm{GmbH}$ ), and by testing appearance of distinct bands for ribosomal RNAs at $\sim 2.3 \mathrm{~kb}$ (28S) and $\sim 1.1 \mathrm{~kb}$ (18S) during agarose gel electrophoresis of $1 \mu \mathrm{l}$ RNA. The extracted RNA was directly used for cDNA synthesis.

\subsubsection{Polymerase chain reaction}

Polymerase chain reaction (PCR) was used for amplification of DNA fragments for construction of plasmids, Southern probes, confirmation of the presence of genes in fungal genomic DNA, verification of gene annotations on cDNA level, and for colony PCRs (Bergkessel \& Guthrie, 2013) to identify E. coli transformants harboring a desired plasmid after plasmid transformation. Phusion High-Fidelity DNA Polymerase, Taq DNA Polymerase (both: Thermo Fisher Scientific), and Q5 High Fidelity Polymerase (New 
England Biolabs) were used for amplification of DNA. PCR programs were designed according to the manufacturer's instructions. Appropriate annealing temperatures of primer pairs were determined by the use of the online NEB Tm calculator v 1.9.12 (New England Biolabs, Ipswich, MA, USA).

\subsubsection{Agarose gel electrophoresis}

Agarose gel electrophoresis was used to separate DNA or RNA according to charge and size for analyses of quality and concentration of purified DNA or RNA. Furthermore, it was used to test specificity of PCR products, to separate genomic DNA fragments cut by restriction enzymes in Southern hybridization analyses, and to purify DNA fragments from unspecific products with subsequent gel extraction. Nucleic acid samples were mixed with $6 x$ loading dye $[0.25 \%$ Bromophenol blue, 0.25\% Xylene cyanol FF, $40 \%$ sucrose, $\mathrm{pH}$ 8.0] and loaded on a $1 \%$ agarose gel [1\% agarose, $1 \times$ TAE buffer ( $40 \mathrm{mM}$ tris-acetate, $20 \mathrm{mM}$ sodium acetate, $2 \mathrm{mM}$ EDTA, $\mathrm{pH} 8.3), 0.001 \mathrm{mg} / \mathrm{ml}$ ethidium bromide] in 1x TAE buffer. The GeneRuler $1 \mathrm{~kb}$ DNA Ladder and GeneRuler $100 \mathrm{bp}$ DNA Ladder (Thermo Fisher Scientific) were used as size standards. Separation was performed with an electric field of $90 \mathrm{~V}$. In-ge/ visualization was achieved by exposure to UV light $(\lambda=254 \mathrm{~nm})$ using a Gel iX20 Imager and the Intas GDS gel documentation software (Intas Science Imaging Instruments $\mathrm{GmbH}$, Goettingen, Germany).

\subsubsection{Abscence confirmation of the VI43LS20kb region in $\mathrm{V}$. longisporum $\mathrm{VI32}$}

Seven genes predicted for the VI43LS20kb region were amplified using Phusion HighFidelity DNA polymerase (Thermo Fisher Scientific) from genomic DNA (2.4.1) of VI43 and VI32 with similar concentrations. Primers are listed in Table 3. A part of the histone H2a encoding genes homologous to V. dahliae JR2 (VDAG_JR2_Chr4g01430a) was amplified with primers SZ19/SZ20 resulting in 854 bp PCR products as positive controls. 1112 bp LSG-1 (VDAG_JR2_Chr5g10950a homolog) was amplified with primers JST229/JST230, 687 bp LSG-2 (VDAG_JR2_Chr2g10300a homolog) with JST231/JST232, $2177 \mathrm{bp} \quad$ LSG-3 (VDAG_JR2_Chr2g10290a homolog) with JST231/JST232, 498 bp LSG-4 (VDAG_JR2_Chr2g10280a homolog) with JST233/JST234, 619 bp LSG-5 (VDAG_JR2_Chr2g10270a homolog) with JST207/JST208, 2448 bp LSG-6 (VDAG_JR2_Chr2g10260a homolog) with JST46/JST50, $527 \mathrm{bp}$ LSG-7 (VDAG_JR2_Chr2g10250a homolog) with JST235/JST236.

\subsubsection{Primer walking}

Primer walking (Sverdlov \& Azhikina, 2005) is a sequencing method used for sequencing of DNA fragments, which extend the sequencing capacities of a single read between 
$1-1.5 \mathrm{~kb}$ obtained from the Sanger sequencing method. The fragment of interest is divided into shorter fragments and sequenced from both ends with specific primers. According to the sequencing result the next sequencing primers are designed in several steps in order to sequence the complete fragment. The method was used to verify the assembly of the $V$. longisporum VI43LS20kb region. The region was divided into four overlapping fragments, which were amplified using primers JST11/JST218 (5538 bp), JST217/JST208 (5900 bp), JST207/JST50 (5140 bp), and JST21/JST13 (5258 bp). The fragments were ligated to the pJet1.2 cloning vector (Thermo Fisher Scientific) for sequencing with the primers pJet1.2-forward/pJet1.2-reverse sequencing primers (Thermo Fisher Scientific), JST15, JST16, JST17, JST220, JST223, JST224, JST228, JST229, JST231, JST234, pKO2-40B-for, and JST238-242.

\subsubsection{Complementary DNA amplification}

RNA was extracted and quality of RNA was determined as described in chapter 2.4.1. Reverse transcription of $0.8 \mu \mathrm{g}$ RNA to cDNA was performed using the Qiagen QuantiTect Reverse Transcription Kit (Hilden, Germany) according to the manufacturer's protocol. Absence of genomic DNA in cDNA samples was tested using $H 2 A$ as a control gene with the primers SZ19/SZ20.

\subsubsection{Quantitative reverse transcriptase PCR}

Transcription of HAC1 was analyzed by quantitative reverse transcriptase (qRT) PCR. RNA was extracted and quality of RNA was determined as described in chapter 2.4.1 from $50 \mathrm{ml}$ liquid SXM cultures inoculated with $1 \times 10^{6}$ spores. Reverse transcription was performed as described in chapter 2.4.6. The cDNA was diluted 1:5. Primers are listed in Table 3 and were designed with annealing temperatures of $60^{\circ} \mathrm{C}$ using the Eurofins Genomics Oligo analysis tool. Histone H2A (VDAG_JR2_Chr4g01430a) and EIF2B (VDAG_JR2_Chr4g00410a) served as reference genes. Transcription levels were analyzed in triplicates using a CFX Connect Real Time System cycler (Biorad) with Mesa Green qPCR MasterMix Plus for SYBR Assay (Eurogentec) and cycling was 2:20 min at $95^{\circ} \mathrm{C}$ followed by 40 cycles of: $95^{\circ} \mathrm{C}$ for $20 \mathrm{~s}, 60^{\circ} \mathrm{C}$ for $22 \mathrm{~s}$, and $72{ }^{\circ} \mathrm{C}$ for $22 \mathrm{~s}$. Specificity of PCR products was tested with the subsequent melting curve analysis from $65^{\circ} \mathrm{C}$ to $95^{\circ} \mathrm{C}$ with $5 \mathrm{~s}$ per $0.5^{\circ} \mathrm{C}$ after $10 \mathrm{~s}$ at $95^{\circ} \mathrm{C}$. Primers JST290/JST291 (HAC1 variants), SZ9/SZ10 (H2A), and SZ11/SZ12 (EIF2B) were used. Expression levels were quantified relative to the reference genes in $\triangle \triangle C T$ method (Livak \& Schmittgen, 2001). $\mathrm{A}$ gene study of two independent experiments was performed using CFX Manager Software version 3.1 (Biorad). 


\subsubsection{Verification of intron-exon structures}

ORFs and intron-exon-structures of the tested genes were analyzed by PCR amplification of transcripts from cDNA. Amplification of the ORF from genomic DNA served as control. Phusion High-Fidelity DNA polymerase was used according to the manufacturer's instructions.

Due to the size of the $4662 \mathrm{bp}$ spliced HAM5 transcript, it was amplified from wild type JR2 cDNA prepared from a four-day-old fungal liquid SXM culture in two overlapping fragments with JST53/JST58 (2476 bp) and JST90/JST45 (3227 bp).

The $1068 \mathrm{bp}$ VMK1 transcript was amplified from cDNA, which was prepared from mycelium obtained from plating of $1 \times 10^{6}$ conidiospores on solid SXM covered with nitrocellulose membrane and incubation for four days. The primers JST61/JST62 were used.

The 1569 bp MEK2 transcript was amplified from cDNA (prepared from a four-day-old fungal liquid SXM culture) using primers JST226/JST292.

The 1446 bp ODE1 transcript was amplified from cDNA (prepared from a six-day-old fungal liquid PDM culture incubated in the dark) using primers JST175/JST176.

The cDNA used for amplification of HAC1 was isolated from wild type cultures grown in $50 \mathrm{ml} \mathrm{SXM}\left(1 \times 10^{7}\right.$ spores $)$ and incubated at $25^{\circ} \mathrm{C}$ under constant agitation for four days for the un-induced HAC1 mRNA variant and with subsequent supplementation with $3 \mathrm{mM}$ DTT for three hours for the induced HAC1 mRNA variant. The $1581 \mathrm{bp}$ un-induced HAC1 mRNA sequence was amplified with primers JST171/JST172. The $1254 \mathrm{bp}$ induced splice variant of HAC1 was amplified using primers JST171/JST174.

PCR fragments were analyzed by agarose gel electrophoresis, excised from gel, and purified using the NucleoSpin Gel and PCR Clean-up Kit (Macherey-Nagel) according to the manufacturer's instructions. Transcripts were fully sequenced. The ORF and splice sites given were confirmed for HAM5 (VDAG_JR2_Chr4g07170a), VMK1 (VDAG_JR2_Chr2g01260a), MEK2 (VDAG_JR2_Chr1g13070a), and ODE1 (VDAG_JR2_Chr1g29610a). The transcript variant given for HAC1 (VDAG_JR2_Chr2g09780a) was confirmed as the uninduced variant HAC1u. A second splice variant of the $H A C 1$ mRNA named $H A C 1^{i}$ was identified (sequence given in Figure S10).

\subsubsection{Southern hybridization}

All Verticillium transformants constructed in this study were confirmed by Southern hybridization analysis (Southern, 1975). Genomic DNA was extracted (2.4.1), cut by appropriate restriction enzymes overnight, and separated by agarose gel electrophoresis. The gel was washed under constant agitation in three steps: for ten 
minutes in Wash buffer $1(0.25 \mathrm{M} \mathrm{HCl}), 25$ min in Wash buffer $2(0.5 \mathrm{M} \mathrm{NaOH}, 1.5 \mathrm{M}$ $\mathrm{NaCl})$ for denaturation, and $30 \mathrm{~min}$ in Wash buffer $3(0.5 \mathrm{M}$ tris, $1.5 \mathrm{M} \mathrm{NaCl}, \mathrm{pH} 7.4)$ for neutralization. The genomic DNA fragments were transferred by dry blotting from the gel onto an Amersham Hybond-Nylon membrane (GE Healthcare). The membrane was dried at $70^{\circ} \mathrm{C}$ for ten minutes and exposed to UV-light $(\lambda=254 \mathrm{~nm})$ for three minutes per side of the membrane for cross-linking of the DNA fragments. For labeling with the probe and subsequent detection, the Amersham Gene Images AlkPhos Direct Labeling and Detection System (GE Healthcare) was used according to the manufacturer's instructions.

The probes were hybridized to DNA fragments on the membrane at $60{ }^{\circ} \mathrm{C}$ rotating overnight. The membrane was washed twice with prewarmed first washing buffer ( $2 \mathrm{M}$ urea, $50 \mathrm{mM}$ SDS, $50 \mathrm{mM} \mathrm{Na}_{3} \mathrm{PO}_{4}, 150 \mathrm{mM} \mathrm{NaCl}, 1 \mathrm{mM} \mathrm{MgCl}$, $\%$ blocking reagent) for ten minutes at $60^{\circ} \mathrm{C}$ rotating, and twice with the second washing buffer ( $1 \mathrm{M}$ tris base, $1 \mathrm{M} \mathrm{NaCl}, \mathrm{pH}$ 10) for five minutes at room temperature under constant agitation. Detection of the labeled DNA bands was performed by CDP-Star Detection Reagent (GE Healthcare) application to the membrane, incubation for $5 \mathrm{~min}$, and exposure of an Amersham Hyperfilm ECL (GE Healthcare). The film was developed using an Optimax (Protec GmbH\&Co.KG) film processor.

\subsection{Protein methods}

\subsubsection{Protein extraction}

For western hybridization, proteins were extracted from mycelium harvested from liquid cultures through Miracloth (Calbiochem Merck) and rinsed with $0.96 \% \mathrm{NaCl}$ solution. Mycelium was dried, frozen, and ground to fine powder in liquid nitrogen using mortar and pestle. The powder was mixed with $B^{*}$ buffer $(300 \mathrm{mM} \mathrm{NaCl}, 100 \mathrm{mM}$ tris- $\mathrm{HCl}$ $\mathrm{pH} 7.5,10 \%$ glycerol, $2 \mathrm{mM}$ EDTA, $0.02 \% \mathrm{NP}_{4} \mathrm{O}$ ), supplemented with $2 \mathrm{mM}$ DTT and $10 \mu \mathrm{l} / \mathrm{ml}$ Protease inhibitor cocktail mix (Roche; stock solution: one tablet in $500 \mu \mathrm{l} \mathrm{B}$ buffer). The mixture was centrifuged for $30 \mathrm{~min}$ at $13000 \mathrm{rpm}$ at $4{ }^{\circ} \mathrm{C}$. The supernatant was transferred to a new tube and used for further analysis or stored at $-20^{\circ} \mathrm{C}$.

\subsubsection{Determination of protein concentration by Bradford assay}

The concentration of proteins was determined by the Bradford assay (Bradford, 1976). The absorbance of the protein sample mixed with Roti-Quant solution (Carl Roth $\mathrm{GmbH} \&$ Co.KG) was measured with an Infinite M200 microplate reader operated with Magellan software (Tecan Trading AG). A bovine serum albumin (BSA; Carl Roth $\mathrm{GmbH} \&$ Co.KG) dilution series was used as standard. 


\subsubsection{SDS-PAGE}

Sodium dodecyl sulfate-polyacrylamide gel electrophoresis (SDS-PAGE; Smith, 1984) was used for separation of protein extracts according to the proteins' molecular masses. The protein extract (2.5.1) was mixed with sample buffer [250 mM tris- $\mathrm{HCl} \mathrm{pH} 6.8,15 \%$ $\beta$-mercaptoethanol, 30\% glycerol, 7\% SDS, 0.3\% Bromophenol blue] and boiled at $95{ }^{\circ} \mathrm{C}$ for five minutes. Prestained Protein Ladder (Thermo Fisher Scientific) was used as standard. Indicated amounts of the denaturized protein extracts were loaded on $12 \%$ acrylamide gels [Running gel: $3.3 \mathrm{ml} \mathrm{H} \mathrm{H}_{2} \mathrm{O}, 2.5 \mathrm{ml}$ tris buffer $\mathrm{pH} 8.8$ (stock: $1.5 \mathrm{M}$ ), $100 \mu \mathrm{l}$ SDS (stock: 10\%), $4 \mathrm{ml}$ polyacrylamide (stock: 30\%), $100 \mu \mathrm{l}$ ammonium persulfate (APS; stock: $10 \%), 10 \mu \mathrm{l} N, N, N^{\prime}, N^{\prime}$-tetramethylethane-1,2-diamine (TEMED); stacking gel: $650 \mu \mathrm{l}$ polyacrylamide (stock: $30 \%$ ), $1.25 \mathrm{ml}$ gel buffer ( $3 \mathrm{M}$ tris, $\mathrm{pH} 8.45,0.3 \%$ SDS), $3 \mathrm{ml} \mathrm{H} \mathrm{H}_{2} \mathrm{O}, 15 \mu \mathrm{l}$ Coomassie Brilliant Blue, $60 \mu \mathrm{l}$ APS (stock: 10\%), $10 \mu \mathrm{l} \mathrm{TEMED],} \mathrm{placed}$ in running buffer (25 mM tris-base, $250 \mathrm{mM}$ glycine, 0.1\% SDS) and run at 120-200 V in Mini-Protean Tetra Cell (Bio-Rad Laboratories $\mathrm{GmbH}$ ) for separation of proteins.

\subsubsection{Immunoblot hybridization}

During immunoblot hybridization, proteins separated by SDS-PAGE (2.5.3) were blotted to a nitrocellulose membrane (Amersham Protran $0.45 \mu \mathrm{m}$, GE Healthcare Life Sciences) using a Mini Trans-Blot Cell (Bio-Rad Laboratories) filled with transfer buffer [25 mM tris-base, $192 \mathrm{mM}$ glycerol, 0.02\% SDS, 20\% methanol]. Wet electroblotting was performed either at $35 \mathrm{~V}$ at room temperature overnight or for one hour at $100 \mathrm{~V}$ with cool packs. PonceauS (0.2\% PonceauS, 3\% TCA) staining was used for visualization of transferred proteins as a loading control. The membrane was washed in TBST buffer [tris buffered saline and Tween20: $50 \mathrm{mM}$ tris, $150 \mathrm{mM} \mathrm{NaCl}, 0.05 \%$ Tween20] and blocking in TBST supplemented with 5\% milk powder (Sucofin TSI GmbH\&Co.KG, Zeven, Germany) for one hour at room temperature was performed to prevent unspecific binding of the antibody. Membranes were incubated in primary antibody a-GFP sc-9996 (1:250; Santa Cruz Biotechnology) or a-HA (1:2000; Sigma-Aldrich Chemie GmbH) in TBST buffer with $5 \%$ milk powder at $4{ }^{\circ} \mathrm{C}$ overnight. Membranes were washed in TBST buffer for ten minutes at room temperature under constant agitation three times. As secondary antibody a-mouse 115-035-003 (1:2000; Jackson Immuno Research) in TBST buffer with $5 \%$ milk powder was applied to the membranes and incubated at room temperature for one to two hours. Membranes were washed in TBST buffer for ten minutes at room temperature under constant agitation three times. Detection of proteins was conducted via horseradish peroxidase (HRP) substrate luminol based chemiluminescence. Two detection solutions (solution 1: $2.5 \mathrm{mM}$ luminol, $400 \mu \mathrm{M}$ paracoumarat, $100 \mathrm{mM}$ tris- $\mathrm{HCl}$ $\mathrm{pH}$ 8.5; solution 2: $100 \mathrm{mM}$ tris- $\mathrm{HCl} \mathrm{pH} \mathrm{8.5,} 5.4 \mathrm{mM} \mathrm{H}_{2} \mathrm{O}_{2}$ ) were applied to the membrane 
and incubated for two minutes at constant agitation in the dark. Signals were detected with the Fusion SL chemiluminescence detector (Peqlab Biotechnology $\mathrm{GmbH}$ ), operated with the corresponding software Fusion 15.18 (Vilber Lourmat), and Amersham Hyperfilm ECL film (GE Healthcare Life Sciences), which was developed by the use of the Optimax (Protec GmbH\&Co.KG) film processor.

\subsection{Sequence analyses}

The online databases National Center for Biotechnology Information (NCBI; Geer et al., 2010), VertiBase (http://biofung.gobics.de:1555/; coordinated by Prof. Dr. Gerhard H. Braus, University of Goettingen), and Ensembl Fungi (Kersey et al., 2018) were used for BLAST searches. Verticillium gene predictions, accession numbers, and sequences were obtained from Ensembl Fungi and VertiBase. Seqbuilder (DNASTAR, Madison, USA) software was used for plasmid and primer design, prediction of restriction sites of enzymes, translation of DNA, and cDNA sequences into amino acid sequences and for calculation of molecular weights of proteins. Appropriate annealing temperatures of primer pairs were determined by the use of the online NEB Tm calculator v 1.9.12 (New England Biolabs, Ipswich, USA) or the Eurofins Genomics Oligo analysis tool for qRTPCR primers. Sanger sequencing of plasmid DNA or PCR products was performed by the Microsynth Seqlab in Goettingen. Obtained sequences were analyzed using Lasergene (DNA STAR INC., Madison, WI, USA) software. The web server RNAfold (Zuker, 2003) was used for determination of secondary structures in qRT-PCR amplicons and mRNAs. Specificity of qRT-PCR primers was predicted by BLAST searches using Ensembl Fungi.

Prediction of protein domains was obtained using the InterPro website (http://www.ebi.ac.uk/Tools/pfa/iprscan; Jones et al., 2014). The presence of localization signals was analyzed using the online databases cNLS mapper (http://nlsmapper.iab.keio.ac.jp/cgi-bin/NLS_Mapper_form.cgi; Kosugi et al., 2009) and DeepLoc-1.0(Almagro Armenteros et al., 2017; http://www.cbs.dtu.dk/services/DeepLoc/i ndex.php). Multiple alignment of protein sequences was performed with ClustalW (Thompson et al., 1994) or Muscle (Edgar, 2004) algorithms in MEGA6.0 software (Tamura et al., 2013). Protein sequence identity matrices were obtained from multiple sequence alignments using the pairwise sequence identity and similarity calculation (SIAS) tool (http://imed.med.ucm.es/Tools/sias.html). Phylogenetic analysis was performed using Maximum likelihood tree calculations with MEGA6.0 software. 


\section{Results}

\subsection{The V. longisporum VI43LS20kb region makes the pathogenic isolate V143 more tolerable for the plant and is absent in the asymptomatic isolate $\mathrm{VI} 32$}

\subsubsection{V. longisporum strains VI43 and VI32 are able to colonize rapeseed plants with different consequences for plant growth and development}

$V$. longisporum species from different lineages display differences in their pathotypes. Isolates from the lineage A1/D1 are the most pathogenic on rapeseed whereas isolates from the lineage A1/D3 are the least pathogenic, but also colonize rapeseed plants (Novakazi et al., 2015). Two isolates of these lineages, VI43 (A1/D1) and VI32 (A1/D3), were isolated from rapeseed fields in the same area in Germany (Zeise \& von Tiedemann, 2002). The question about the genomic basis for the establishment of different pathotypes in related species was addressed, focusing on a Lineage Specific region of the pathogenic $V$. longisporum isolate VI43, which is absent in the genome of the asymptomatic isolate $\mathrm{VI} 32$.

In initial experiments, the colonization behavior of the two $\mathrm{V}$. longisporum isolates VI43 and VI32 in rapeseed plants was compared by monitoring of induced disease symptoms and subsequent analysis of fungal outgrowth from infected tissue. One-week-old rapeseed plants were inoculated by root dipping into the same numbers of spores obtained from VI43, VI32, or into the water control (mock). After 35 days only VI43 induced visible disease symptoms in the plants, including decreased height and leave length as well as chlorosis and necrosis of the leaves and reduced weight (Figure 7A). About $27 \%$ of the plants died from fungal infection with strain VI43, 30\% displayed strong symptoms, $27 \%$ weak symptoms and $17 \%$ of the tested plants did not show any disease symptoms. Plants infected with strain VI32 were unaffected and did not show any alterations compared to uninfected mock plants.

Previous studies revealed that symptomatic and asymptomatic $V$. longisporum isolates show only minor lineage-specific phenotypic differences on specific media ex planta. No differences in early plant colonization on $A$. thaliana roots were observed regarding their growth on the root surface and formation of hyphopodia as invasion structures (unpublished data of Rebekka Harting from the University of Goettingen and Rabea Schlüter from the University of Greifswald).

Re-isolation experiments from surface sterilized stem sections of rapeseed plants inoculated with either one of the two $V$. longisporum isolates were conducted to test if both strains are able to further colonize the plant and show differences in the colonization 
behavior after initial root penetration. After seven days strong fungal outgrowth was visible from VI43 infected stem sections and to a smaller extend also from plants treated with VI32 spores (Figure 7B).

Both $V$. longisporum isolates are able to colonize the vascular system of rapeseed plants, but only VI43 induces plant disease, whereas rapeseed colonization by VI32 is asymptomatic.

A
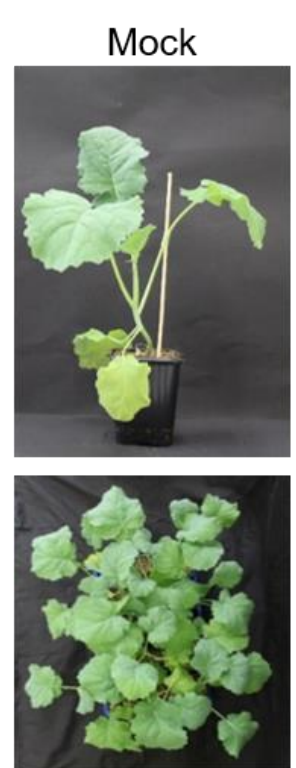

B

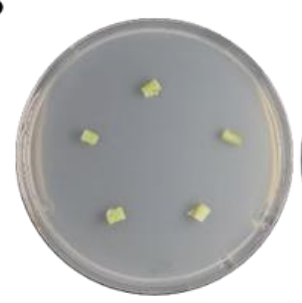

VI43
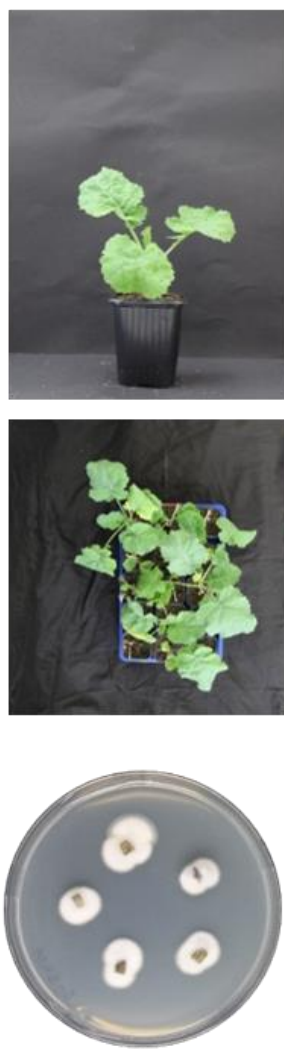

VI32
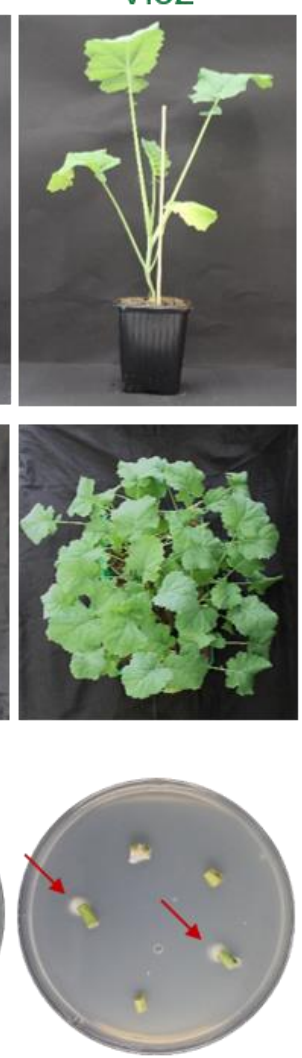

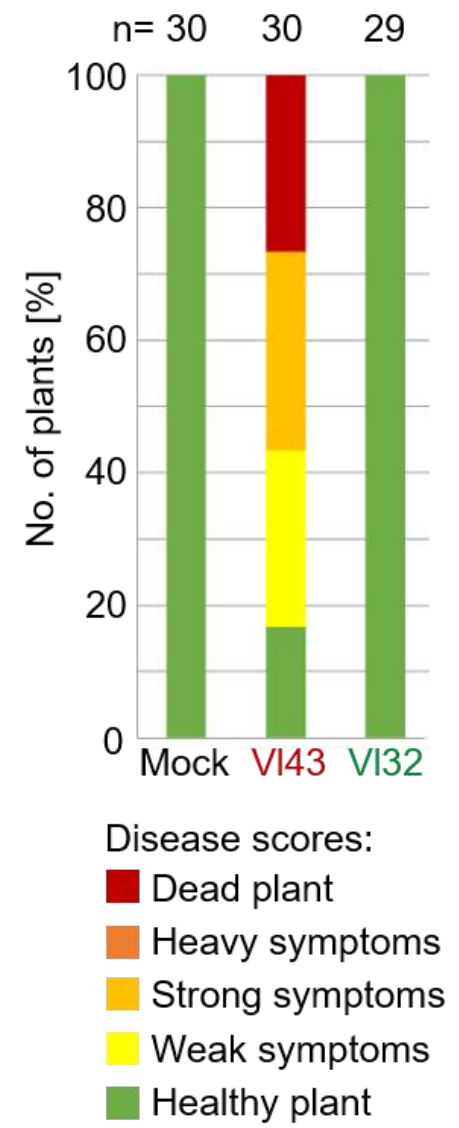

Figure 7: V. longisporum strains VI43 and VI32 colonize B. napus plants either pathogenically or asymptomatically. (A) Seven-day-old B. napus seedlings were inoculated by root dipping into distilled water as control (mock) or the same number of spores $\left(1.5 \times 10^{6}\right.$ spores $\left./ \mathrm{ml}\right)$ obtained from VI43 and VI32. Disease symptoms were scored after 35 days. Single representative $B$. napus plants (top) and overview pictures of 15 plants per strain from a single experiment (bottom) 35 days post inoculation are shown. Parameters fresh weight, height of vegetation point, length of longest leaf, and symptom index of leaves were assessed in two independent experiments and transformed into disease scores per plant relative to control plants. The stack diagram shows the relative number of plants with certain disease scores. $\mathrm{n}=$ total number of evaluated plants. $V$. longisporum strain VI43 induced visible disease symptoms in B. napus plants, whereas no disease symptoms can be observed in plants inoculated with VI32 spores. (B) Fungal outgrowth from stem sections of representative plants treated with sterile water (mock), VI43, or VI32 spores. Stems were harvested 35 days post inoculation with fungal spores, surface sterilized, cut, and incubated on PDM supplemented with chloramphenicol for seven days at $25^{\circ} \mathrm{C}$. Both fungal strains could be re-isolated from surface sterilized infected stem sections on plate, however, to a smaller extend from plant material inoculated with VI32 spores (indicated by red arrows). 


\subsubsection{The pathogenic V. Iongisporum VI43 genome harbors the VI43LS20kb region, which is absent in the asymptomatic colonizer VI32}

In order to investigate on which genomic basis the different pathotypes of the two $V$. longisporum isolates rely, the genomes of the pathogen and the asymptomatic colonizer were sequenced (BioFung Consortium BMBF). It was found that only very few genes were specific for one of the two $V$. longisporum genomes. Optical mapping of both genomes revealed differences in the chromosome numbers with 16 for VI32 and 15 for VI43, correlating with alterations in the chromosome sizes. Genome alignments allowed to explain the differences in the karyotypes between the two $V$. longisporum isolates by massive syntenic rearrangements as previously observed in several species of the genus Verticillium (Shi-kunne \& Faino, 2017). A correlation between genomic rearrangements and the evolution of LS regions was found in haploid Verticillia (Klosterman et al., 2011; de Jonge et al., 2013; Faino et al., 2016; Chen et al., 2018; Gibriel et al., 2019). Klosterman and coworkers reported on LS regions in the genome of $V$. dahliae VdLs.17 that share no synteny with the genome of $V$. alfalfae VaMs.102, which were suggested to be involved in adaptation to different host niches (Klosterman et al., 2011).

In order to address the question if LS genes are important for the pathotype of the $V$. longisporum isolate VI43, a bioinformatic screen was used to identify regions present specifically in the genome of the pathogenic V. longisporum isolate VI43, but are absent in the asymptomatic plant colonizer VI32. Thereby, a region of approximately $20 \mathrm{~kb}$ in size was identified, which is absent in the genome of the asymptomatic A1/D3 isolate. This region is named VI43LS20kb in the following. The same region could be identified in the genome of the pathogenic $V$. longisporum A1/D1 isolate VI145c.

$V$. longisporum species evolved by hybridization from two parental lineages with a $V$. dahliae strain, or at least a close relative to $V$. dahliae, as one of them (Karapapa et al., 1997; Collins et al., 2003; Clewes et al., 2008; Collado-Romero et al., 2010; Inderbitzin et al., 2011b; Tran et al., 2013). A homologous region to the VI43LS2Okb region could be identified in the genome of the $V$. dahliae strain JR2 (Figure 8), but not in the genome of $V$. alfalfae VaMs.102.

Correct genome assembly of the $V$. longisporum VI43LS20kb region was confirmed by PCR amplification and sequencing by primer walking for the entire region. The absence of this region in the VI32 genome, proposed by the bioinformatic screen, was confirmed by PCR reactions targeting seven genes, named Lineage Specific region Gene (LSG) 1 to 7, predicted for the VI43LS20kb region according to the gene annotations for V. dahliae JR2 (Ensembl Fungi, de Jonge et al., 2012; Figure 8). PCR products for all seven LSGs and the histone encoding gene $H 2 A$ as positive control were obtained using VI43 genomic DNA as a template, whereas no specific PCR products were obtained from genomic DNA of $\mathrm{VI32}$ for the seven LSGs, but for the positive control (Figure 8A). 
All predicted genes of this region were only identified in single copy in the $V$. longisporum VI43 and VI145c genomes by BLAST search of the nucleotide sequences. In contrast, the $V$. dahliae JR2 genome harbors two copies for LSG1 identified by BLAST search of the genomic sequence. One copy located in the VI43LS20kb homologous region between VDAG_JR2_Chr2g10300a and VDAG_JR2_Chr2g10310a was not annotated. A second copy is located on chromosome five (VDAG_JR2_Chr5g10950a).

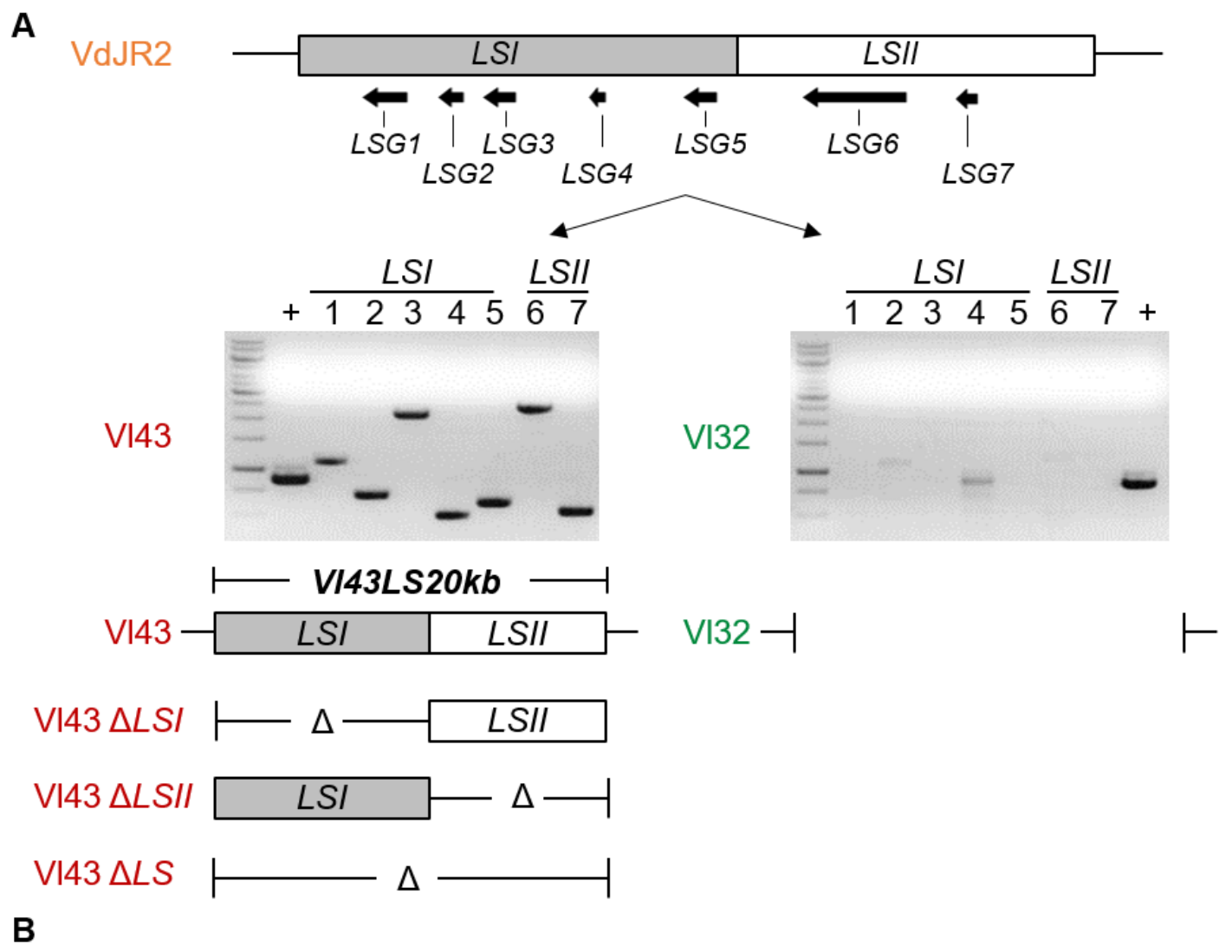

\begin{tabular}{|c|c|c|c|c|c|}
\hline $\begin{array}{l}\text { VI43 } \\
\text { genes }\end{array}$ & $\begin{array}{l}\text { Protein } \\
\text { size } / \mathrm{Mw}\end{array}$ & Domains & Localization & $\begin{array}{l}\text { Vd JR2 homolog/s } \\
\text { (VDAG_JR2_) }\end{array}$ & aa sequ. ID \\
\hline LSG1 & $173 \mathrm{aa} / 19 \mathrm{kDa}$ & - & Cytosol & $\begin{array}{l}\text { Chr5g10950a, } \\
2^{\text {nd }} \text { between } \\
\text { Chr2g10300a/ } \\
\text { Chr2g10310a }\end{array}$ & $\begin{array}{l}100 \% \text { to both } \\
\text { VdJR2 } \\
\text { copies }\end{array}$ \\
\hline LSG2 & $228 \mathrm{aa} / 25 \mathrm{kDa}$ & - & Cytosol & Chr2g10300a & $100 \%$ \\
\hline LSG3 & $258 \mathrm{aa} / 29 \mathrm{kDa}$ & $\begin{array}{c}\text { bHLH } \\
\text { DNA binding }\end{array}$ & Nucleus & Chr2g10290a & $99.2 \%$ \\
\hline LSG4 & $131 \mathrm{aa} / 15 \mathrm{kDa}$ & - & Nucleus & Chr2g10280a & $100 \%$ \\
\hline LSG5 & $163 \mathrm{aa} / 18 \mathrm{kDa}$ & $\begin{array}{l}\text { Zinc finger RING- } \\
\text { type, TM-helix }\end{array}$ & $\begin{array}{l}\text { Plasma } \\
\text { membrane }\end{array}$ & Chr2g10270a & $94.7 \%$ \\
\hline LSG6 & $666 \mathrm{aa} / 74 \mathrm{kDa}$ & bZIP & Nucleus & Chr2g10260a & $99.7 \%$ \\
\hline LSG7 & $119 \mathrm{aa} / 13 \mathrm{kDa}$ & - & Nucleus & Chr2g10250a & $99.2 \%$ \\
\hline
\end{tabular}


Figure 8: The symptomatic V. longisporum strain VI43 harbors a specific VI43LS20kb region in its genome, which is absent in the asymptomatic isolate VI32. (A) Lineage Specific region of $V$. longisporum VI43. An LS region of approximately $20 \mathrm{~kb}$ in size, named VI43LS20kb, is present in the genome of the pathogenic V. longisporum isolate VI43, but absent from the genome of the asymptomatic rapeseed colonizer VI32. A VI43LS20kb homologous region was identified in the genome of $V$. dahliae JR2. Seven Lineage Specific region Genes (LSG1-7) were predicted for this region in V. dahliae (Ensembl Fungi). The presence of the $V$. longisporum VI43LS20kb region in VI43 was confirmed by PCR reactions, giving products for LSG1-7 and the histone $\mathrm{H} 2$ a encoding gene as a positive control (+). No specific PCR products were obtained from genomic DNA of VI32 for the genes predicted in the VI43LS20kb region, but for the positive control. The VI43LS20kb region was dissected into two parts $L S I$ and $L S I I$ and VI43 deletion strains lacking either $L S I, L S I$, or the total $V I 43 L S 20 k b$ region (VI43 $\Delta L S I, \Delta L S I I, \Delta L S)$ were constructed to analyze the role of this region in virulence of VI43. (B) Characteristics of VI43 LSG1-7 deduced proteins. The VI43LS20kb region encodes homologs to LSG1-7 predicted in V. dahliae JR2 (Ensembl Fungi). Protein sequences of the deduced VI43 proteins Lsg1-7 were proposed according to the VI43 genomic sequences (VertiBase) and the V. dahliae JR2 transcripts (Ensembl Fungi). Protein domains were predicted by InterProScan. A basic helix-loop-helix (bHLH) DNA binding domain was proposed for Lsg3 (184-255 aa, IPR011598) as well as a Zinc finger RING-type domain (65-148 aa, IPR013083) and a transmembrane (TM) helix (15-37 aa, Phobius, TM-helix; 40-163 aa cytosolic; N-terminus non-cytosolic) for Lsg5. A basic-leucine zipper (bZIP) domain was predicted for Lsg6 (137-200 aa, PS50217, IPR004827). Protein localizations were predicted using CNLS mapper and DeepLoc-1.0 tools. The amino acid sequence identities (ID) of the VI43 and V. dahliae JR2 proteins were determined by alignment of the amino acid sequences using NCBI tools and MEGA 6.0 software. Mw: molecular weight.

For six of the seven genes in the VI43LS20kb region, the deduced proteins are rather small with 119-258 amino acids (aa) in length (Figure 8B). Only LSG6 potentially encodes a larger protein with 666 aa and a molecular weight of $74 \mathrm{kDa}$. Domains were only predicted for three of the hypothetical proteins. Two of them contain specific transcription factor domains with a basic helix-loop-helix (bHLH) DNA binding domain for Lsg3 and a basic-leucine Zipper (bZIP) domain for Lsg6. For the potential transcription factors Lsg3 and Lsg6, nuclear localization was proposed by the cNLS mapper and DeepLoc-1.0 prediction tools.

Lsg5 contains a Zinc finger RING-type domain and a transmembrane domain. Zinc finger RING-type domains are involved in protein-protein interactions and in mediating ubiquitin transfer to substrates or the Zinc finger RING-type domain protein itself (Joazeiro \& Weissman, 2000). DeepLoc-1.0 proposed a localization of Lsg5 to plasma membranes with $54 \%$.

As for Lsg3 and Lsg6, nuclear localization was predicted for Lsg4, whereas Lsg1 and Lsg2 proteins were sorted to the cytosol (Figure 8B). For none of the hypothetical proteins secretion signals were identified.

In summary, bioinformatic analyses identified a VI43LS2Okb region in the genome of the pathogenic $V$. longisporum isolate VI43, which is absent in the asymptomatic strain VI32. The presence of a homologous region in the haploid $V$. dahliae JR2 corroborates its origin in the parental lineage D1. The VI43LS20kb region encodes six small proteins 
without secretion signals and one larger protein, where two of them have the potential to function as transcription factors and one protein is potentially involved in ubiquitination.

\subsubsection{The VI43LS20kb region reduces virulence in rapeseed plants}

V143 deletion strains were constructed to unravel whether the VI43LS20kb region, which is present in the genome of the pathogenic $V$. longisporum isolate but absent in the asymptomatic isolate $\mathrm{VI} 32$, contributes to the differences in pathotypes.

The VI43LS20kb region was dissected into a $\sim 11.5 \mathrm{~kb} L S I$ region and a $\sim 8.5 \mathrm{~kb} L S I I$ region and VI43 deletion strains lacking either $L S I, L S I$, or the total $L S$ region were constructed (Figure 8A). Correct replacement of the regions against the respective marker cassettes was verified by Southern hybridization (Figure S1). The resulting strains VI43 $\Delta L S I$, VI43 $\Delta L S I I$, and VI43 $L L S$ displayed unaltered ex planta phenotypes in comparison to wild type 14 days after spot inoculation under different growth conditions, including simulated xylem medium, minimal medium with different carbon sources (sucrose or galactose), and minimal medium supplemented with different stress inducing agents as Sorbitol, SDS, or $\mathrm{H}_{2} \mathrm{O}_{2}$ (Figure S2). Small alterations in melanization of the colony centers between independent experiments were not significant.

Infection studies were performed with VI43 $\Delta L S I, \Delta L S I I$, and $\Delta L S$ strains in comparison to the $V$. longisporum isolates VI43, VI32, or water control (mock) to determine the role of the VI43LS20kb genomic region in virulence of VI43. Rapeseed plants treated with spores obtained from the isolate VI32 were indistinguishable from uninfected plants, whereas plants inoculated with VI43 $\Delta L S I, \Delta L S I I$, and $\Delta L S$ spores displayed more severe disease symptoms compared to plants treated with spores from the pathogenic wild type VI43 after 35 days (Figure 9). Whereas $27 \%$ of the plants treated with the VI43 wild type died from fungal infection, $70 \%$ dead plants were scored for VI43 $\Delta L S I, 83 \%$ for VI43 $\Delta L S I I$, and $89 \%$ for VI43 $\Delta L S$ (Figure 9A, B). Significant differences between plants inoculated with spores from VI43 and the LSI, LSII, and LS deletion strains were determined for the numbers of dead plants (Figure 9C).

Reversely, the region identified upon comparison of a pathogenic and an asymptomatic $V$. longisporum isolate does not promote induction of disease symptoms by the pathogen VI43, but attenuates pathogenesis in the host-fungus interaction and makes the fungus more tolerable for rapeseed plants. 
A

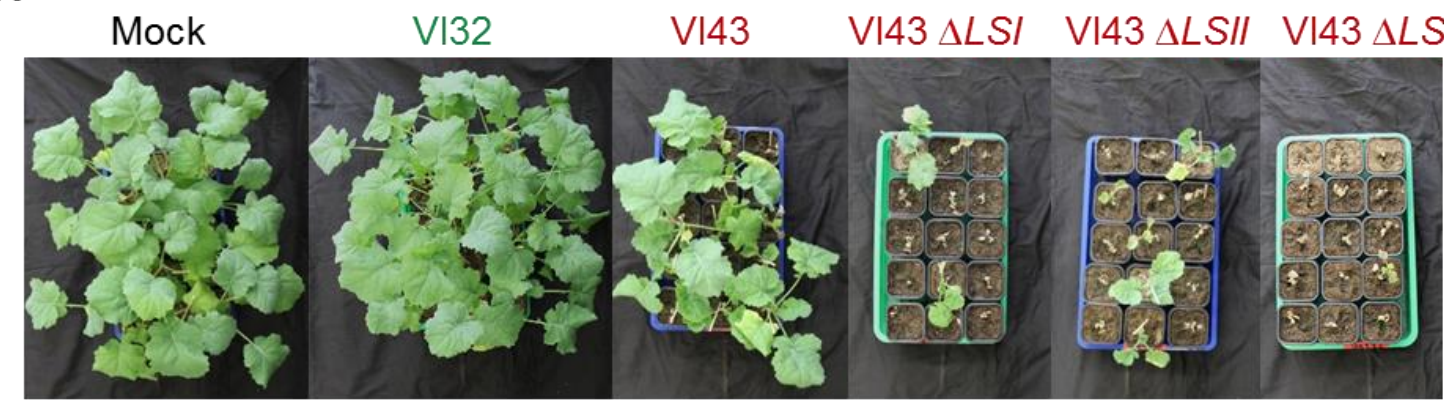

B

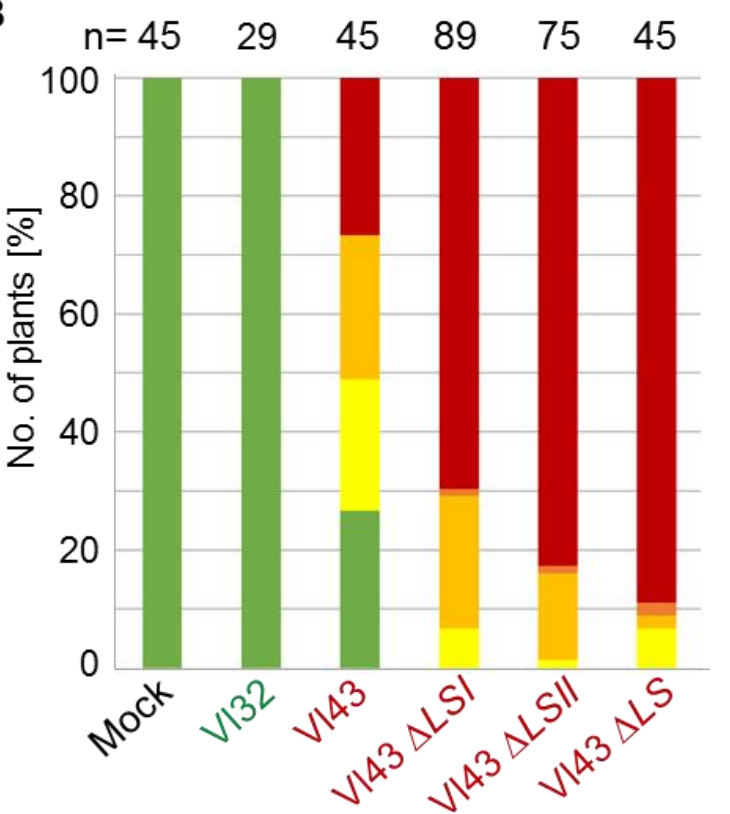

C

Disease scores:

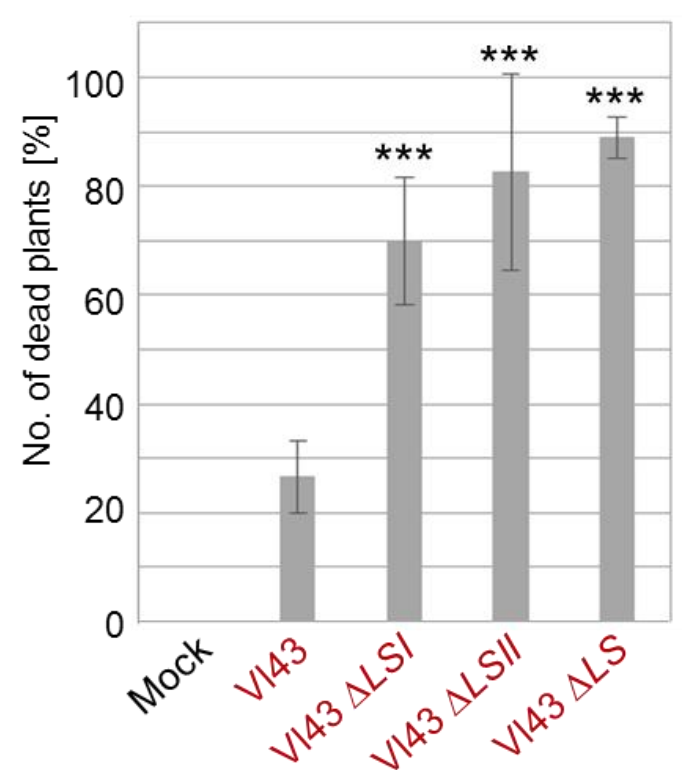

Dead plant

Heavy symptoms

Strong symptoms
Weak symptoms

Healthy plant

Figure 9: The VI43LS20kb region absent in the asymptomatic $V$. longisporum isolate VI32 attenuates disease symptom induction of VI43 in B. napus. Seven-day-old $B$. napus seedlings were inoculated by root dipping into water as control (mock) or into the same numbers of spores obtained from VI32 and VI43 wild types, VI43 $\Delta L S I, \Delta L S I$, or $\Delta L S$. Disease symptoms were assessed after 35 days. Two independent transformants were tested for VI43 $\Delta L S I$ (VGB201, VGB233) and $\Delta L S I I$ (VGB193, VGB214), and a single transformant for $\Delta L S$ (VGB232) in three independent experiments. For plants treated with spores obtained from VI32 no disease symptoms were observed. LSI, LSII and LS deletion strains display increased severity of disease symptoms in comparison to wild type VI43. (A) Overview pictures of $B$. napus plants after 35 days. (B) Stack diagram of the relative number of plants with certain disease scores per genotype. Parameters fresh weight, height of vegetation point, length of longest leaf, and symptom index of leaves were assessed and transformed into disease scores per plant relative to control plants. $n=$ total number of evaluated plants. (C) The diagram shows the number of dead plants 35 days post inoculation with water control or fungal spores relative to the total number of evaluated plants. Bars represent mean values of three independent experiments with standard deviations. Significant differences compared to wild type VI43 were calculated with one-way Anova and Student's t-test: ${ }^{* *} \mathrm{p}<0.001 ; \mathrm{n}($ mock, VI43, $\Delta L S)=3 ; \mathrm{n}(\mathrm{VI} 43 \Delta L S I, \Delta L S I)=6$. 


\subsubsection{The homologous VI43LS20kb region in V. dahliae is indifferent for virulence towards tomato plants}

The VI43LS20kb region identified by comparison of two $V$. longisporum isolates is in the same synteny in the haploid $V$. dahliae strain JR2, suggesting its origin in the haploid parental lineage D1. To address the question if the homologous region in $V$. dahliae has a similar function in attenuation of plant disease symptoms as in V. longisporum VI43, JR2 LS deletion strains were constructed (Figure S3) and tested regarding induction of disease symptoms in tomato plants (Figure 10).

Prior to the investigation of the in planta phenotype, the ex planta phenotypes of $V$. dahliae JR2 $\triangle L S$ were tested in comparison to the wild type ten days after spot inoculation on the same media as VI43 LS deletion strains (Figure S4). Like in $V$. longisporum $\mathrm{VI} 43$, the $L S$ region is dispensable for vegetative growth of $V$. dahliae JR2 ex planta since no significant phenotypic alterations were observed. Only slight reductions in melanization were visible for the JR2 LS deletion strain compared to wild type on CDM with galactose as carbon source (Figure S4).
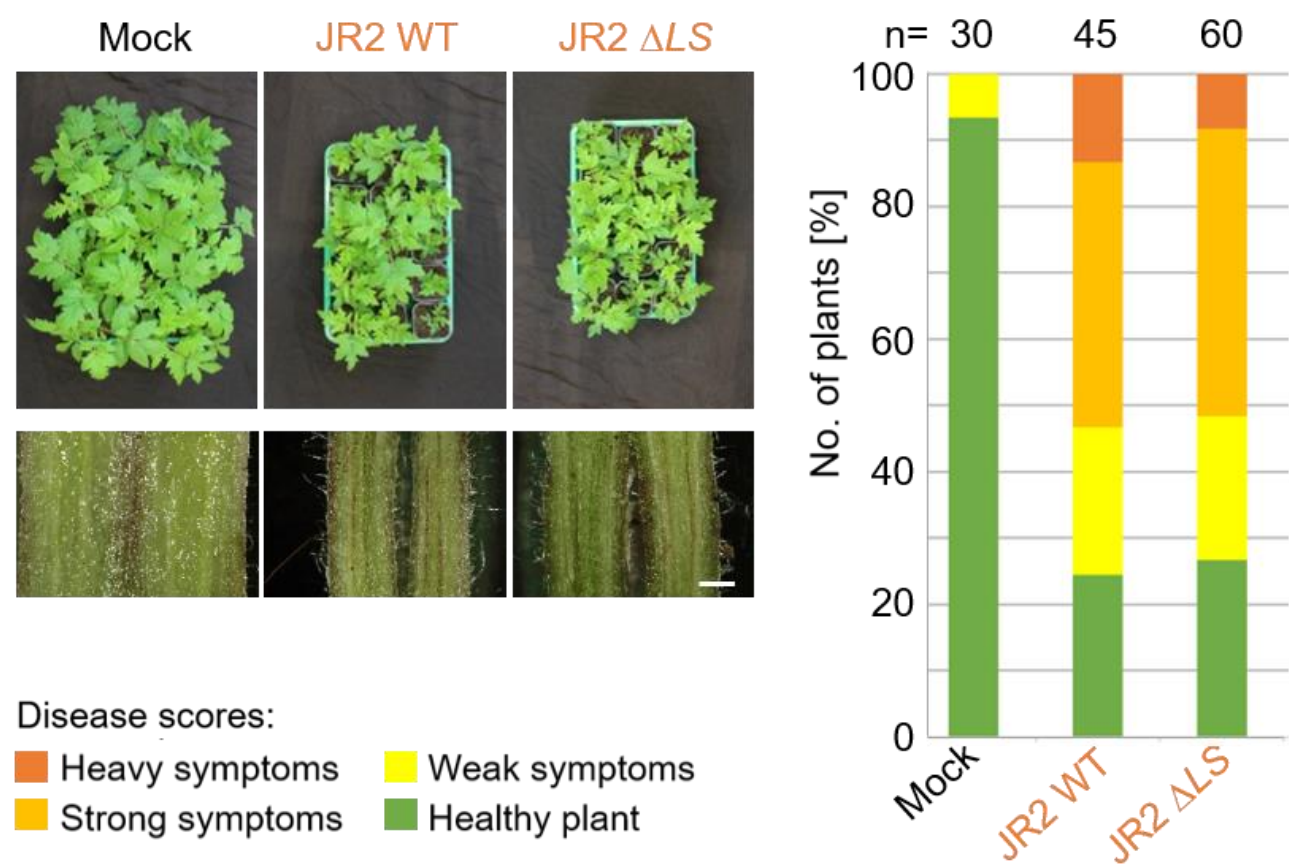

Figure 10: The VI43LS20kb homologous region in V. dahliae is indifferent for virulence towards tomato plants. Ten-day-old $S$. lycopersicum seedlings were inoculated by root dipping into water control (mock), the same numbers of spores obtained from the V. dahliae wild type JR2, or two independent LS deletion transformants (VGB383, VGB393). Overview pictures of control plants and plants inoculated with wild type or JR2 $\Delta L S$ and hypocotyl cross sections of representative plants are shown (Scale bar $=1 \mathrm{~mm}$ ). Disease symptoms fresh weight, height of vegetation point, and length of longest leave were assessed after 21 days in two independent experiments and transformed into a disease score per plant. The stack diagram shows the relative number of plants with a certain disease score. Plants infected with wild type or JR2 $\Delta L S$ display similar severity of disease symptoms. $\mathrm{n}=$ total number of evaluated plants. 
Tomato plant infections were performed to investigate the role of the $V$. dahliae VI43LS20kb homologous region in pathogenicity. S. lycopersicum plants were inoculated with spores obtained from two independent $\Delta L S$ transformants and the disease symptoms were scored after 21 days. JR2 $\Delta L S$ treated tomato plants displayed unaltered severity of disease symptoms and hypocotyl discolorations comparable to wild type infected plants (Figure 10).

Taken together, the $V$. longisporum A1/D1 VI43LS20kb region absent in the asymptomatic $V$. longisporum A1/D3 isolate VI32 is consistent in $V$. dahliae JR2 and presumably derives from the D1 parental strain. The region encodes several small proteins, two potential transcription factors and one protein potentially involved in proteinprotein interactions. In $V$. dahliae, the presence or absence of this region results in similar severity of disease symptoms in tomato plants. In contrast, the same region attenuates disease symptoms induced by the pathogenic $V$. longisporum A1/D1 isolate VI43 in rapeseed. In conclusion, even pathogenic fungi possess specific genomic regions which make virulence more tolerable for the host plant.

\subsection{Vmk1/Mek2 MAPK cascade-mediated microsclerotia formation and pathogenicity in $V$. dahliae are independent from the scaffold protein Ham5}

\subsubsection{V. dahliae Ham5 corresponds to the $N$. crassa scaffold protein HAM-5}

The Vmk1 MAPK signaling cascade is homologous to yeast Fus3/Kss1 MAPK pathways and is essential for regulation of virulence in V. dahliae (Rauyaree et al., 2005; Sarmiento-Villamil et al., 2018). The MAPK pathway components Vmk1 and the yeast Ste12-like transcription factor Vph1 of $V$. dahliae are essential for regulation of pathogenicity and differ in their impact on microsclerotia formation (Rauyaree et al., 2005; Sarmiento-Villamil et al., 2018). The genomes of the Pezizomycotina, the biggest subdivision of the Ascomycota, harbor a gene encoding a highly conserved protein which was allocated to scaffolding functions in the Fus3/Kss1-like MAPK pathway (JametVierny et al., 2007; Dettmann et al., 2014; Jonkers et al., 2014, 2016; Frawley et al., 2018). A scaffold protein for this pathway has not yet been described in any filamentous phytopathogen. The role of the $N$. crassa HAM-5 homolog in differentiation and virulence of $V$. dahliae was analyzed combined with the characterization of the MAP2K homolog Mek2 and the MAPK Vmk1 as control.

The V. dahliae gene VDAG_JR2_Chr4g07170a was identified as a predicted homolog to the $N$. crassa scaffold HAM-5 encoding gene using reciprocal BLAST search of the deduced amino acid sequences against the $V$. dahliae JR2 (Ensembl Fungi) and the 
N. crassa OR74A (NCBI database) proteomes. The HAM5 pre-mRNA of 4906 bp has a predicted intron-exon structure with five exons, which was confirmed by PCR amplification of the spliced transcript from wild type cDNA (Figure 11A).

A

V. dahliae

HAM5
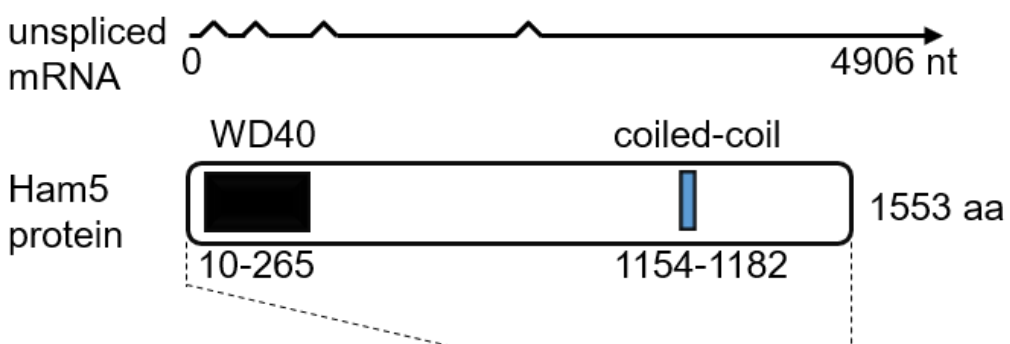

B

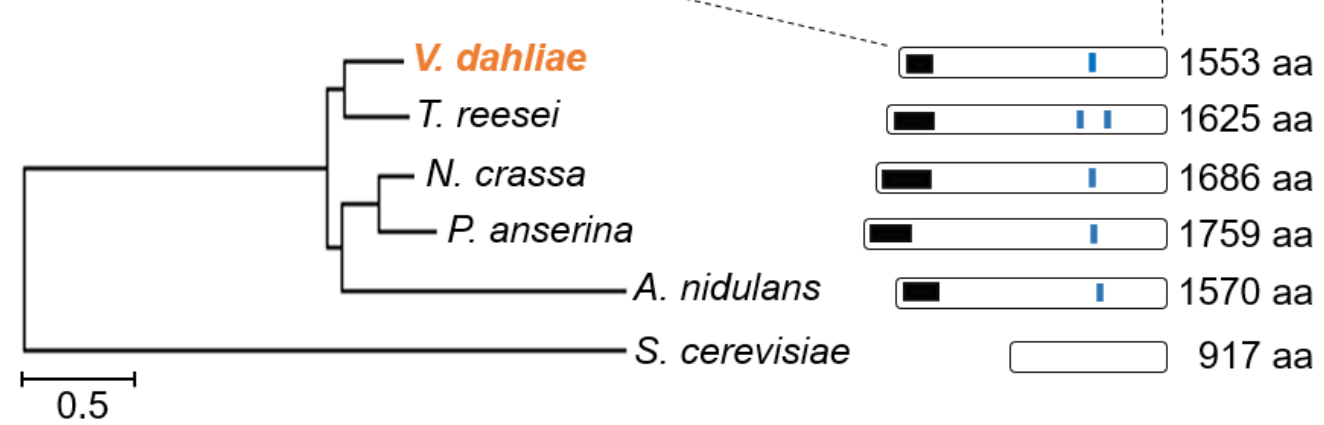

C

Ham5 protein:

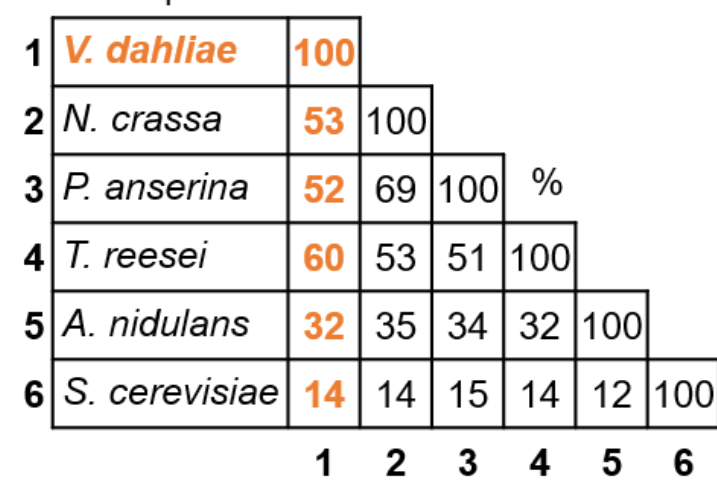

WD40 domain:

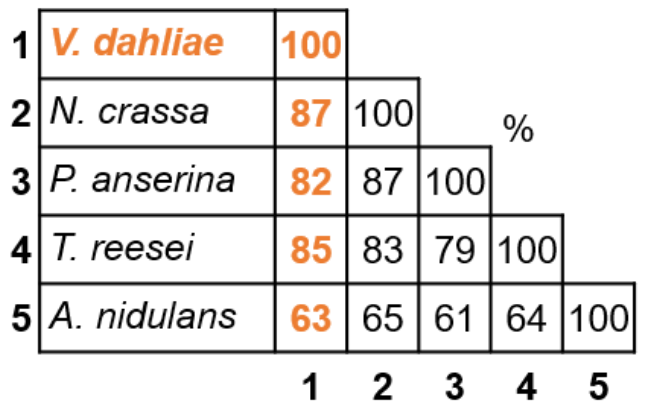

Figure 11: Comparison of $V$. dahliae Ham5 and corresponding proteins in related fungi. (A) The intron-exon structure of $V$. dahliae HAM5 predicted by Ensembl Fungi (VDAG_JR2_Chr4g07170a) was confirmed by PCR amplification of the spliced transcript from wild type cDNA. The protein consists of 1553 aa and contains predicted WD40 repeats at the N-terminus (black; IPR015943; 10-265 aa) and a coiled-coil domain (blue; 1154$1182 \mathrm{aa}$ ). Domains were predicted by InterProScan. (B) Phylogenetic tree of the Ham5 protein. Phylogenetic analysis with aa sequences from: V. dahliae Ham5 (VDAG_JR2 Chr4g07170a), T. reesei HAM-5 (AKN58846.1), N. crassa HAM-5 (XP_011393509.1), P. anserina IDC1 (ABJ96338.2), A. nidulans HamE (AN2701), and S. cerevisiae Ste5 (NP_010388.1) was performed (ClustalW algorithm). WD40 (IPR015943; black box) and coiled-coil domains (blue bar) were predicted by InterProScan. Scale bar = average number of amino acid substitutions per site. (C) Ham5 protein sequence identity matrices of the Ham5 full-length protein homologs and orthologs (left) and their WD40 domains (right) (ClustalW). Amino acid sequence identities are given in \%. Pairwise sequence identity and similarity calculation from multiple sequence alignments were performed using SIAS tool. 
The deduced Ham5 protein with a length of 1553 aa and a molecular weight of $170 \mathrm{kDa}$ contains $\mathrm{N}$-terminal WD40 repeats and a coiled-coil domain at the C-terminus. The corresponding HAM-5 proteins of related filamentous fungi are similar in length and show conserved protein domains, including C-terminal coiled-coil domains and N-terminal WD40 repeat domains (Figure 11A, B). WD40 repeat domains have been found in several fungal scaffold proteins (Pöggeler \& Kück, 2004; Jain, 2019; Hicks et al., 2001). These domains form symmetrical disc-like Beta-propeller structures, shaped by blades assembled around a central channel (Chen et al., 2011) that allow the coordination of multi-protein complexes (Xu \& Min, 2011). The WD40 domain was shown to be important for binding of the MAPK MAK-2 to the scaffold HAM-5 in N. crassa (Jonkers et al., 2014). A phylogenetic analysis of the $V$. dahliae Ham5 protein, its described homologs in ascomycetes, and the non-homologous yeast scaffold protein Ste5 indicated that V. dahliae Ham5 has the highest similarity to Trichoderma reesei HAM-5 with $60 \%$ aa sequence identity, followed by N. crassa HAM-5 with $53 \%$ and Podospora anserina IDC1 with $52 \%$ (Figure 11B, C). V. dahliae Ham5 and A. nidulans HamE are less closely related, showing only $32 \%$ aa sequence identity (Figure 11B, C). The orthologous yeast scaffold protein Ste5 has $14 \%$ aa sequence identity with the $V$. dahliae protein, is much smaller, and does not share the domain predictions of the Ham5 homologs (Figure 11B, C).

The proposed WD40 domains of the corresponding Ham5 proteins in related fungi displayed generally higher amino acid sequence similarities than the full-length proteins (Figure 11C). The deduced protein sequence of the putative HAM-5 homolog in V. dahliae has $87 \%$ identity to N. crassa HAM-5. V. dahliae Ham5 displays higher amino acid sequence conservation of the entire protein and the WD40 domain to N. crassa HAM-5 than A. nidulans HamE which shows $35 \%$ aa sequence identity to the entire protein and $65 \%$ to the WD40 repeat domain of $N$. crassa HAM-5 (Figure 11C).

\subsubsection{The V. dahliae MAP2K Mek2 and the MAPK Vmk1 show high similarities to N. crassa MEK-2 and MAK-2}

The $N$. crassa scaffold protein HAM-5 assembles the MAPK cascade components MAK-2 (MAPK), MEK-2 (MAP2K), and NRC-1 (MAP3K) (Dettmann et al., 2014; Jonkers et al., 2014, 2016). The $V$. dahliae homologs to $N$. crassa mek-2 and the $V$. dahliae VdLs.17 MAPK encoding gene VMK1 were identified by reciprocal BLAST search of the deduced amino acid sequences against the $V$. dahliae JR2 (Ensembl Fungi), N. crassa OR74A (NCBI database), or the V. dahliae VdLs.17 (Ensembl Fungi) proteome. 
The potential MAP2K homolog in $V$. dahliae is encoded by the gene VDAG_JR2_Chr1g13070a. The V. dahliae MEK2 pre-mRNA of 1756 bp contains four exons (Figure 12A left).
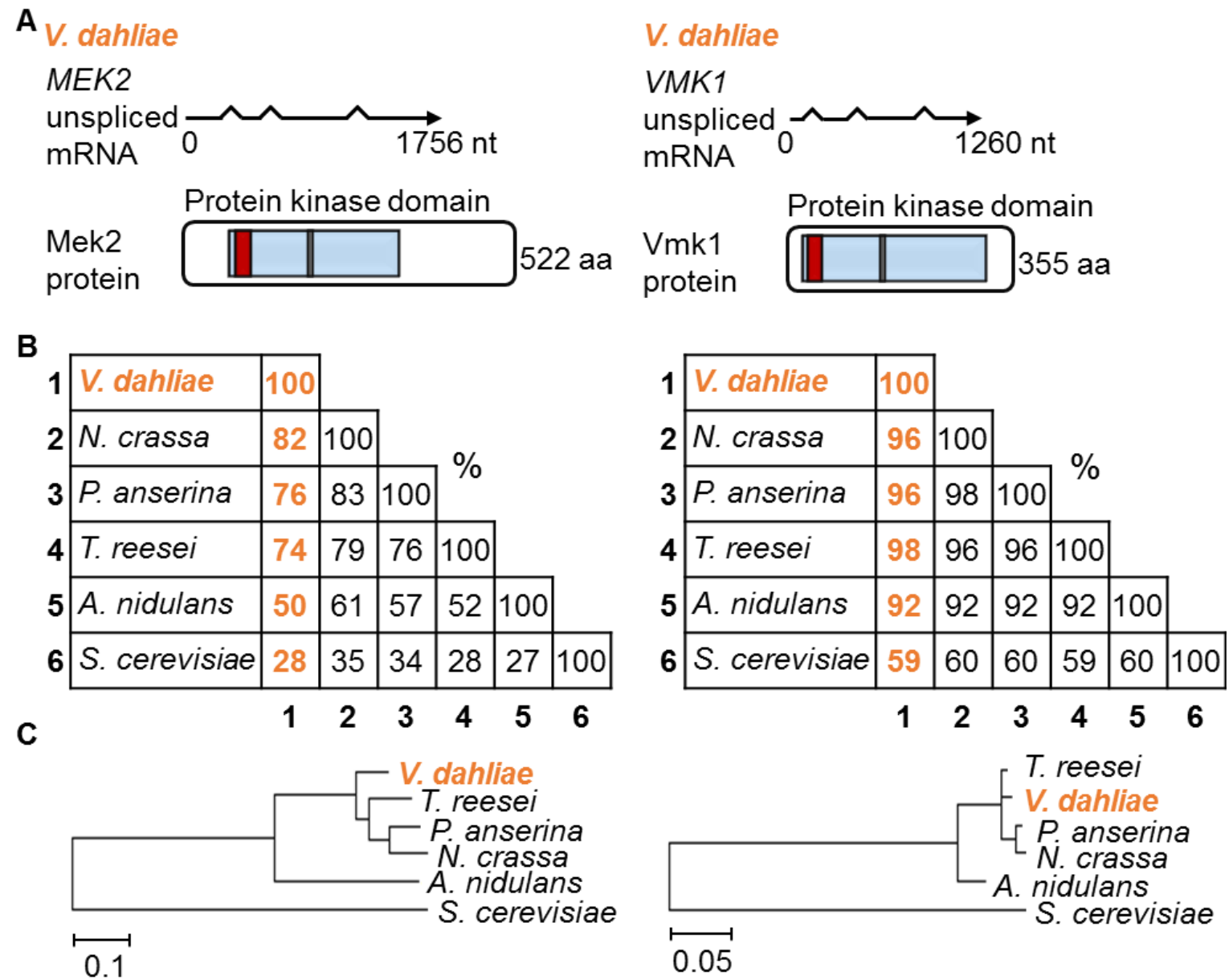

Figure 12: The V. dahliae MAP2K Mek2 and MAPK Vmk1 are highly conserved in fungi. (A) The unspliced mRNA structure and protein domain predictions of $V$. dahliae Mek2 and Vmk1. Predicted intron-exon structures of the $1756 \mathrm{bp} \mathrm{MEK2} \mathrm{(left)} \mathrm{and} \mathrm{the} 1260 \mathrm{bp} \mathrm{VMK1}$ (right) unspliced mRNAs were confirmed by PCR amplification of spliced transcripts from wild type cDNA. Mek2 consists of 522 aa and contains a protein kinase domain (blue; 67-332 aa; IPR000719), with the serine/threonine-protein kinase active site at 186-198 aa (grey bar; IPR008271) and an ATP binding site (red; 73-96 aa; IPR017441). Vmk1 consists of 355 aa and contains a protein kinase domain (blue; 23-311 aa; IPR000719), with the serine/threonine-protein kinase active site at 143-155 aa (grey bar; IPR008271) and an ATP binding site (red; 29-53 aa; IPR017441). Domains were predicted by InterProScan. (B) Protein sequence identity matrices of corresponding Mek2 (left) and Vmk1 (right) proteins from related fungi. Protein sequence identities are given in \%. Identifiers of used sequences are given in C. ClustalW algorithm, pairwise amino acid sequence identity and similarity calculation were performed using the SIAS online tool. (C) Phylogenetic trees of corresponding Mek2 (left) and Vmk1 (right) proteins from related fungi. Left: protein sequences were used from: V. dahliae Mek2 (VDAG_JR2_Chr1g13070a), T. reesei (XP_006963390.1), P. anserina (XP_001910826.1), N. crassa Mek-2 (XP_011394665.1), A. nidulans MkkB (AN3422), S. cerevisiae Ste7 (NP 010122.1). Right: prōein sequences were used from: V. dahliae Vmk1 (VDAG JR2_Chr2g01260a), T. reesei tmk1

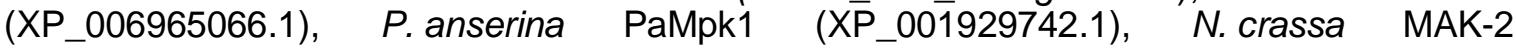
(AAK 25816.1$), \quad$ A. nidulans MpkB (AAF12815.2), $\quad$ S. cerevisiae Fus3 (NP_009537.1) (ClustalW algorithm). Scale bar = average number of amino acid substitutions per site. 
Expression was confirmed by PCR amplification of the spliced transcript from wild type cDNA. The deduced protein Mek2 consists of 522 aa with a molecular weight of $57 \mathrm{kDa}$ and contains a protein kinase domain with a serine/threonine-protein kinase active site and an ATP binding site (Figure 12A left). V. dahliae Mek2 possesses high conservation with maximum $82 \%$ aa sequence identity to $N$. crassa $M E K-2$ and only $28 \%$ to the yeast S. cerevisiae MAP2K Ste7 (Figure 12B, C left).

The homologous gene to VdLs.17 VMK1 (VDAG_09461) was identified in V. dahliae JR2 (VDAG_JR2_Chr2g01260a) with 100\% aa sequence identity. The predicted VMK1 premRNA of 1260 bp contains four exons (Figure 12A, right) and expression was confirmed by PCR amplification of the spliced transcript from wild type cDNA. The deduced protein Vmk1 consists of 355 aa with a molecular weight of $41 \mathrm{kDa}$. A protein kinase domain with a serine/threonine-protein kinase active site and an ATP binding site was predicted by InterProScan (Figure 12A, right).

The $V$. dahliae MAPK Vmk1 possesses even higher conservation than the MAP2K with $98 \%$ aa sequence identity to the corresponding protein in T. reesei, followed by $96 \%$ to the respective MAP kinase in $N$. crassa and $P$. anserina (Figure 12B, C, right).

Both components of the yeast Fus3-like MAPK pathway, the MAPK Vmk1 and the MAP2K Mek2, are highly conserved in V. dahliae JR2, with Mek2 a little less conserved in analyzed species than Vmk1.

\subsubsection{Vmk1- and Mek2-mediated regulation of vegetative growth and microsclerotia formation is independent from Ham5}

In order to analyze the role of the putative scaffold Ham5 in Vmk1 MAPK pathwaymediated development and virulence of $V$. dahliae, HAM5, MEK2, and VMK1 deletion strains as well as HAM5/MEK2 and HAM5/VMK1 double deletion strains were constructed. The genes under control of their native promoters and terminators were ectopically reintegrated into the genomes of the single deletion strains to analyze whether complementation of phenotypes is possible. Prior to functional investigation, correct integration of deletion and complementation constructs was confirmed by Southern hybridization analysis (Figure S5, S6, S7).

Analysis of the ex planta phenotype of the HAM5 deletion strain revealed wild type-like growth. No alterations were observed regarding colony size, melanization, or microsclerotia formation under several tested conditions, including complete media (PDM, Malt extract agar), simulated xylem medium, or minimal medium with different carbon sources (CDM with either sucrose, galactose, glucose, or cellulose). In Figure 13A $\triangle$ HAM5 colonies nine days after spot inoculation on SXM and CDM with either sucrose or cellulose as carbon source are shown as examples. 


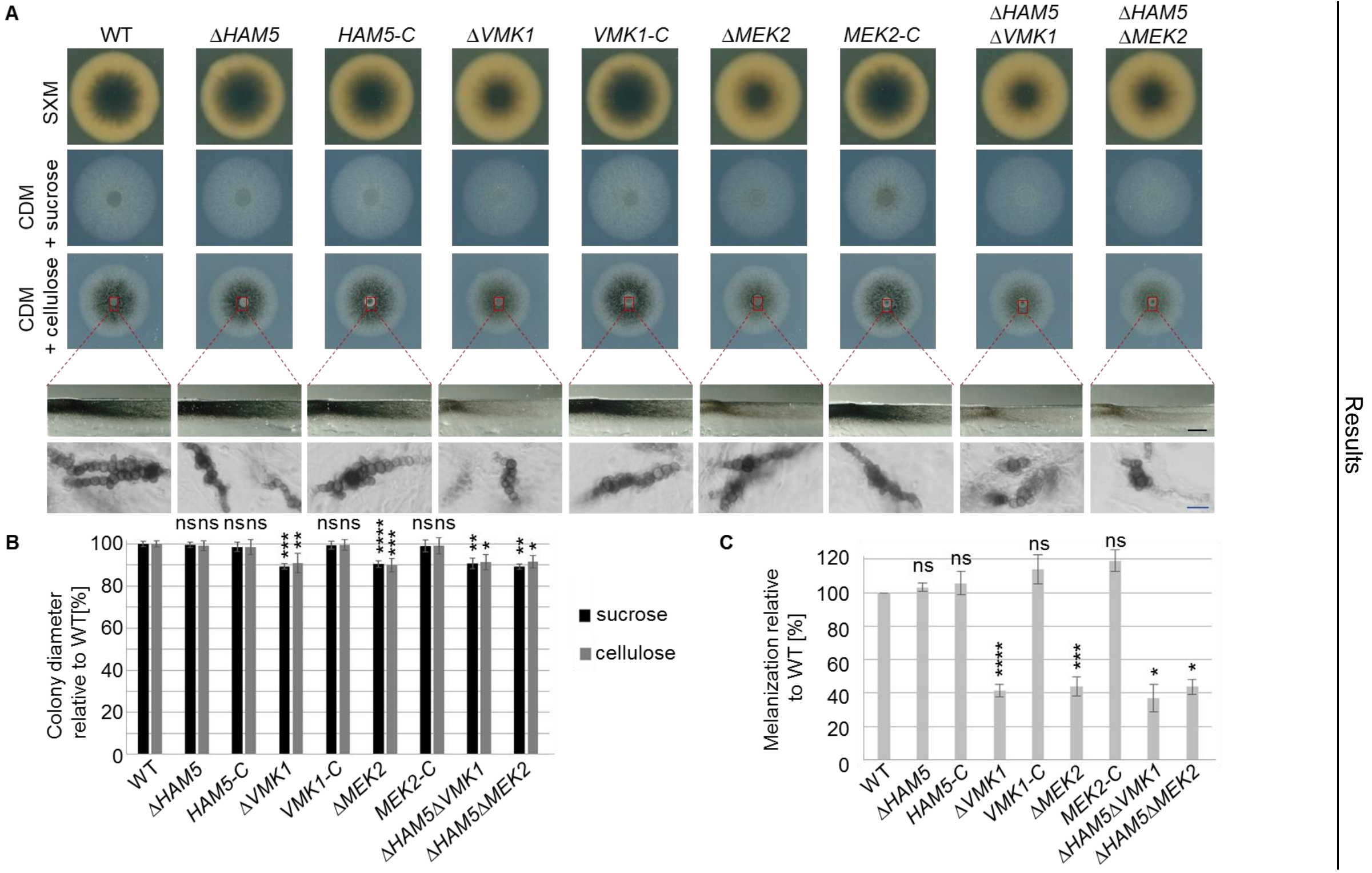


Figure 13: V. dahliae VMK1- and MEK2-mediated vegetative growth and microsclerotia formation function independent of HAM5. The HAM5 deletion strain was compared to wild type JR2, VMK1, and MEK2 single deletions with respective complementation strains, as well as $\triangle H A M 5 \triangle V M K 1$ and $\triangle H A M 5 \triangle M E K 2$ double deletions regarding their vegetative growth and microsclerotia formation ex planta. 50000 freshly harvested spores were spot inoculated on SXM or CDM with either sucrose or cellulose as carbon source and incubated at $25^{\circ} \mathrm{C}$ for nine days. The ex planta phenotypes of two independent transformants per single deletion strain (VGB279, VGB280, VGB335, VGB336, VGB337, VGB338) or one transformant per double deletion (VGB346, VGB417) and complementation strain (VGB389, VGB413, VGB415) were tested. Black scale bar $=1 \mathrm{~mm}$, blue scale bar $=20 \mu \mathrm{m}$. (A) Ex planta phenotypes nine days after spot inoculation. Cross sections of colony centers and microscopy of fungal material scraped from colony centers grown on CDM with cellulose as carbon source (indicated by red boxes and dashed lines) are shown. (B) Growth quantification on either CDM with sucrose (black) or cellulose (grey) as carbon source nine days after spot inoculation. Growth of $\triangle V M K 1, \triangle M E K 2, \triangle H A M 5 \triangle V M K 1$, and $\triangle H A M 5 \triangle M E K 2$ strains is significantly decreased to about $90 \%$ in comparison to wild type. $\triangle H A M 5$ and the complementation strains show wild type-like growth. Growth was quantified by measurement of two perpendicular diameters per colony for three plates per transformant and medium $(n=1)$. Bars represent mean values of three independent experiments with standard deviations relative to wild type. Significant differences to wild type determined with one-way Anova and Student's t-test are shown with ${ }^{*} \mathrm{p}<0.05 ;{ }^{* *} \mathrm{p}<0.01 ;{ }^{* \star *} \mathrm{p}<0.001$, ${ }^{* \star * *} \mathrm{p}=0$, ns $=$ nonsignificant. $\mathrm{n}(\triangle H A M 5 ; \triangle V M K 1 ; \triangle M E K 2)=6 ; \mathrm{n}(\mathrm{WT} ; \triangle H A M 5 \triangle V M K 1 ; \triangle H A M 5 \triangle M E K 2 ; H A M 5-C$; $V M K 1-C ; M E K 2-C)=3$. (C) Melanization of colonies grown on CDM with cellulose relative to wild type. Whereas $\triangle H A M 5$ shows wild type-like melanization, $\triangle M E K 2, \triangle V M K 1$, $\triangle H A M 5 \triangle M E K 2$, and $\triangle H A M 5 \triangle V M K 1$ display a significant decrease to $38-46 \%$ of the wild type level. Pictures were taken from the top view of the colonies after removal of aerial mycelium. The brightness factor of the colony center was measured for three colonies per transformant $(n=1)$ using the ImageJ software. The means of the brightness factor were determined, set relative to wild type, and inverted to obtain melanization values. Bars represent mean values with standard deviations from two independent experiments. Significant differences to wild type determined with one-way Anova and Student's t-test are shown with ${ }^{*} p<0.05$; ${ }^{* \star \star} \mathrm{p}<0.001,{ }^{* \star \star *} \mathrm{p}=0$, ns $=$ non-significant. n(WT; $\triangle H A M 5 \triangle V M K 1 ; \triangle H A M 5 \Delta M E K 2 ;$ HAM5$C ; V M K 1-C ; M E K 2-C)=2 ; \mathrm{n}(\triangle H A M 5)=3 ; \mathrm{n}(\triangle V M K 1 ; \triangle M E K 2)=4$.

Furthermore, vegetative growth of the $\triangle H A M 5$ strain was tested on minimal medium supplemented with stress inducing agents $\left(0.5 \mathrm{M} \mathrm{NaCl}, 0.00075 \% \mathrm{H}_{2} \mathrm{O}_{2}, 0.004 \%\right.$ SDS, or $0.8 \mathrm{M}$ Sorbitol) without observation of any phenotypical alterations. Since no selfanastomosis of vegetative hyphae or germination tubes were observed for the $V$. dahliae wild type JR2 under any growth condition, the role of Ham5 in cell fusion was not characterized.

In contrast to the HAM5 deletion strain, MEK2 and VMK1 single and double deletion strains show a $\sim 10 \%$ significant decrease in growth on CDM with either sucrose or cellulose as carbon source nine days after spot inoculation which was not observed for the complementation strains MEK2-C and VMK1-C (Figure 13A, B).

Whereas the HAM5 deletion strain shows wild type-like melanization, the colony centers formed by strains $\triangle M E K 2, \triangle V M K 1, \triangle H A M 5 \triangle M E K 2$, and $\triangle H A M 5 \triangle V M K 1$ on SXM and CDM with sucrose or cellulose as carbon source appear less melanized after nine days (Figure 13A). The differences in melanization become more apparent in cross sections of the colony centers. Microscopy of fungal material from the colony centers for all tested strains showed melanized microsclerotia similar to wild type, however, in qualitatively 
reduced frequencies in $\triangle M E K 2, \triangle V M K 1, \triangle H A M 5 \triangle M E K 2$, and $\triangle H A M 5 \triangle V M K 1$ (Figure $13 A)$. The melanization of the colony centers of all strains grown on cellulose containing medium was quantified and confirmed the wild type-like melanization in the $\triangle H A M 5$ strain. In contrast, a significant decrease in melanization to less than half was observed for VMK1 and MEK2 single or double deletion strains (Figure 13C).

These data corroborate, that MAP2K MEK2 and MAPK VMK1 contribute to vegetative growth, microsclerotia formation, and melanization independently from the putative cascade scaffold protein Ham5 in V. dahliae.

\subsubsection{Mek2- and Vmk1-mediated regulation of virulence is independent from Ham5 in tomato plants}

The impact of the putative scaffold protein Ham5 on virulence of $V$. dahliae was investigated in tomato plant infection experiments in comparison to the MAP2K Mek2 and the MAPK Vmk1.

Tomato plants inoculated with spores from $\triangle M E K 2, \triangle V M K 1, \triangle H A M 5 \triangle M E K 2$, or $\triangle H A M 5 \triangle V M K 1$ strains displayed plant heights comparable to mock plants after 21 days and no hypocotyl discolorations were observed (Figure 14A). None of the plants inoculated with spores from these strains displayed strong or heavy symptoms. The avirulent in planta phenotypes observed for $\triangle M E K 2$ and $\triangle V M K 1$ were complemented in experiments with VMK1-C and MEK2-C strains. Plants treated with VMK1-C displayed slightly more severe disease symptoms with only $3 \%$ healthy plants, whereas MEK2-C and wild type treatment resulted in about $20 \%$ healthy plants (Figure 14A).

In contrast to MEK2 and VMK1 single or double deletion strain treated plants, tomato plants inoculated with $\triangle H A M 5$ displayed disease symptoms indistinguishable from wild type infected plants including severe stunting and discoloration of the vascular system (Figure 14A).

To investigate which of the constructed strains are able to colonize plant tissues, reisolation from stem sections on agar plates was performed. Fungal outgrowth from stems was observed for all strains, even if to a smaller extend from plants treated with spores from $\triangle V M K 1, \triangle M E K 2, \triangle H A M 5 \triangle V M K 1$, and $\triangle H A M 5 \triangle M E K 2$ strains than from plants treated with wild type, $\triangle H A M 5$, or complementation strains (Figure 14B). For strains causing no symptoms, fungal outgrowth was confirmed by control PCR reactions targeting the transit region of the integrated marker cassettes (Figure 14C). Hence, MEK2 and VMK1 single and double deletion strains are able to invade tomato plants, but are impaired in induction of disease symptoms, whereas deletion of HAM5 allows wild type-like induction of disease symptoms. 
A
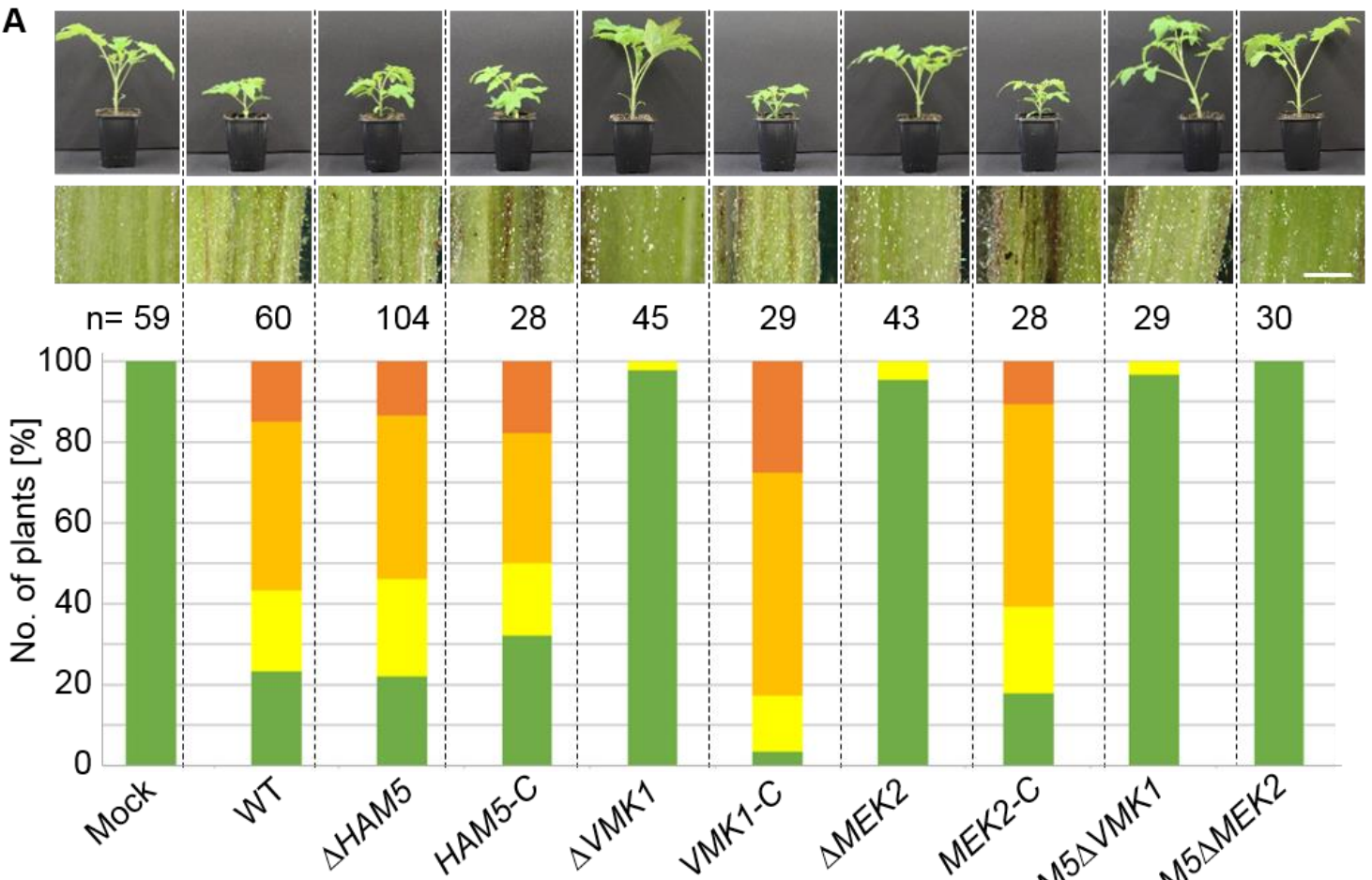

Disease scores:

Healthy plant

Weak symptoms

Strong symptoms
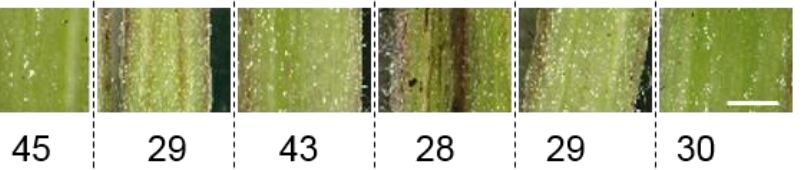

28

29

30
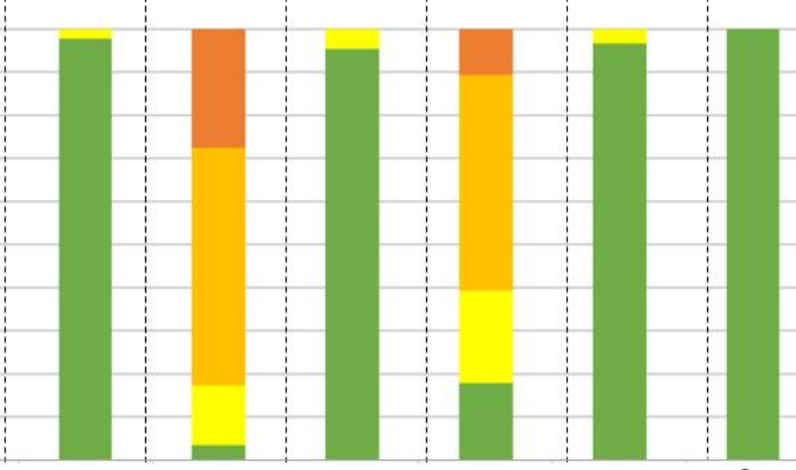

B
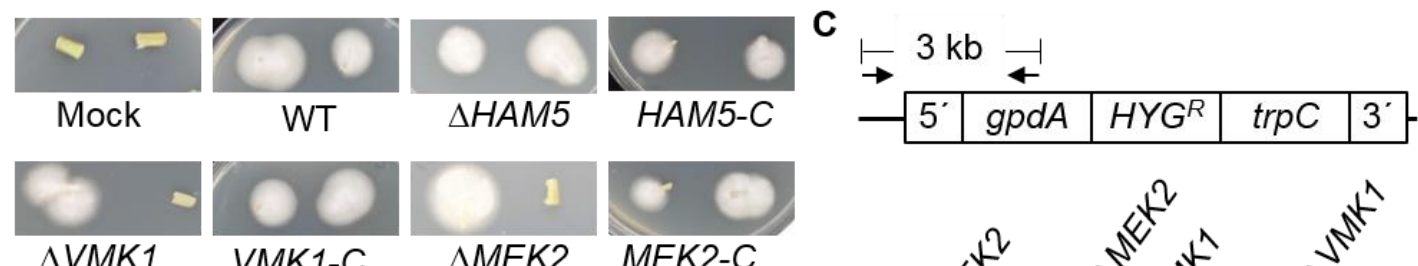

$\triangle V M K 1$
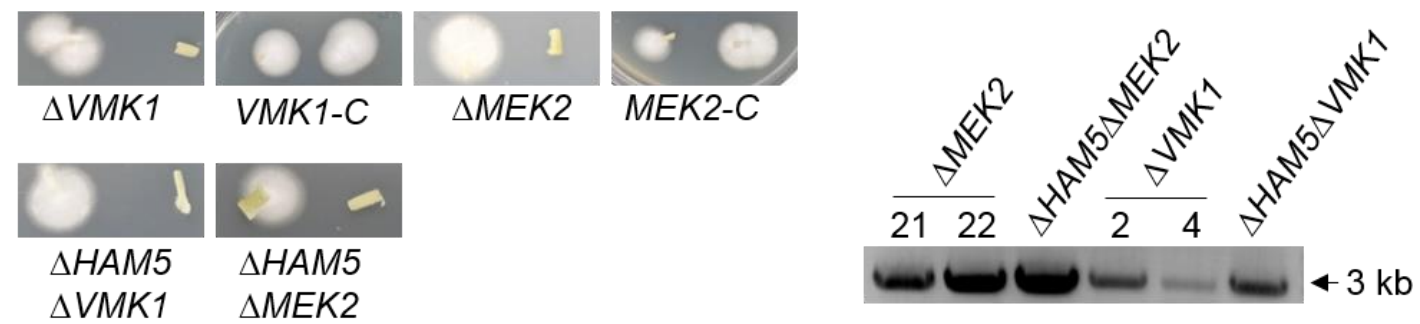

Figure 14: V. dahliae Vmk1 pathway-mediated pathogenicity towards S. lycopersicum is independent from the scaffold protein. (A) Ten-day-old tomato plants were inoculated by root dipping into sterile water as control (mock) or spores obtained from wild type JR2 (WT), HAM5, VMK1, and MEK2 deletions with the respective complementations (HAM5-C, VMK1-C, and MEK2-C) or double deletion strains. Representative plants and hypocotyl dissections after 21 days are shown. The relative number of plants with certain disease scores are displayed in the stack diagram. Two independent transformants of $\triangle H A M 5$ were tested in three (VGB280) to four (VGB279) independent experiments. Two independent transformants per VMK1 (transformant numbers 2 and 4 - VGB335, VGB336) and MEK2 (transformant numbers 21 and 22 - VGB337, VGB338) deletion and a single transformant per double deletion (VGB346, VGB417) and complementation strain (VGB389, VGB413, VGB415) were tested in two independent experiments. $n=$ the total number of evaluated plants; scale bar $=1 \mathrm{~mm}$. (B) Stem assay shows fungal outgrowth from stem sections for all tested strains. Stems were surface sterilized, cut, and incubated on PDM supplemented with chloramphenicol for seven days at $25^{\circ} \mathrm{C}$. (C) PCR validation of fungal outgrowth from stem assay as VMK1 and MEK2 single and double deletion strains. PCR reactions targeting the regions upstream of the $5^{\prime}$-flanking region of $V M K 1$ or MEK2 used for homologous recombination and the gpdA promoter of the introduced hygromycin resistance marker cassette give products with predicted fragment length about $3 \mathrm{~kb}$ for genomic DNA of all tested strains. 
These data corroborate that the function of the MAPK cascade components Vmk1 and Mek2 in virulence are independent from the scaffold protein Ham5. This was also observed for the MAPK pathway functions in vegetative growth and microsclerotia formation. Therefore, the cascade either requires a yet unidentified scaffold protein or a direct interplay between the kinases for the tested conditions.

\subsection{The unfolded protein response pathway regulator Hac1 controls development and virulence of $V$. dahliae}

\subsubsection{HAC1 and IRE1 genes of the UPR pathway in V. dahliae}

Efficient biosynthesis and secretion of proteins involved in colonization of the host plant are crucial for the success of plant colonizing fungi and assumed to be regulated by the unfolded protein response pathway (Joubert et al., 2011; Heimel et al., 2013). The UPR pathway senses ER stress correlated with increased protein folding demands via the transmembrane endoribonuclease Ire1 and regulates responsive genes via the bZIP transcription factor Hac1. In the course of this study, the presence of the fundamental components of the UPR pathway in $V$. dahliae was examined by BLAST search of deduced amino acid sequences and the function of the HAC1 homolog in development and virulence of $V$. dahliae was characterized.

The gene VDAG_JR2_Chr1g29650a was identified as V. dahliae JR2 IRE1 homolog by reciprocal BLAST search with the T. reesei Ire1 protein sequence of 1243 aa (XP_006962552.1, isolate QM6a). Incorrect annotation of the ORF with a deduced protein length of only 871 aa in $V$. dahliae JR2 is likely. Using the annotation of the homologous gene in VdLs.17 (VDAG_02974), the presence of an ORF encoding a 1236 aa protein with proposed Ire1-specific domains, including an ER luminal domain, a protein kinase, and a cytosolic endoribonuclease domain, was identified (Figure 15A). The deduced Ire1 proteins from $V$. dahliae and T. reesei display $56 \%$ aa sequence identity.

The V. dahliae HAC1 homolog VDAG_JR2_Chr2g09780a was identified by reciprocal BLAST search with the deduced amino acid sequence of $A$. nidulans hacA (Q8TFU8.2, isolate FGSC A4) against the $V$. dahliae JR2 (Ensembl Fungi) and A. nidulans (NCBI database) proteomes. The gene contains an ORF of $1634 \mathrm{bp}$. The Ensembl Fungi gene annotation of the potential HAC1 homolog in $V$. dahliae predicted only one splice variant. The HAC1 homologs in related filamentous fungi possess two mRNA splice variants, an uninduced $H A C 1^{u}$ and an unconventionally spliced $H A C 1^{i}$ mRNA which is preferentially spliced in response to ER stress (Heimel, 2015). Therefore, the hypothetically deduced protein of 526 aa with a molecular weight of $58 \mathrm{kDa}$ was named Hac1 $1^{\mathrm{u}}$. For Hac1 ${ }^{\mathrm{u}}$ an $\mathrm{N}$ terminal basic-leucine zipper domain and an N-terminal nuclear localization signal (NLS) 
were predicted (Figure 15B). The deduced Hac1 ${ }^{\mathrm{u}}$ proteins from $V$. dahliae and T. reesei display $35 \%$ aa sequence identity.

Hence, the $V$. dahliae genome harbors $H A C 1$ and IRE1 homologs with similar deduced protein sequences and domain predictions to the corresponding proteins in related filamentous fungi.

A

Ire1

protein endoribo-

V. dahliae

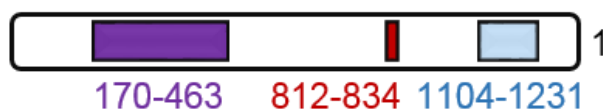

T. reesei

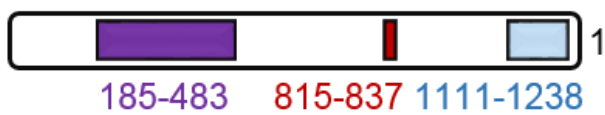

B Hac1u 1236 aa

V. dahliae 1243 aa

A. nidulans

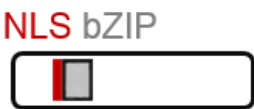

$107-164$

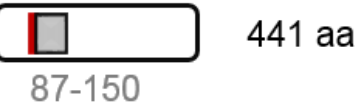

Figure 15: Protein domains of V. dahliae Ire1 and Hac1u in comparison to corresponding proteins of related filamentous fungi. Domains of deduced protein sequences of homologous IRE1 (VDAG_02974/VDAG_JR2_Chr1g29650a corrected) and HAC1 (VDAG_JR2_Chr2g09780a) genes in V. dahliae in comparison to the corresponding proteins in related filamentous fungi predicted by InterProScan and CNLS mapper. (A) Domain predictions for V. dahliae Ire1 (1236 aa protein; ER luminal Ire1 domain, cd09769, 170-463aa; protein kinase ATP binding site, IPR017441, 812-834 aa; cytosolic C-terminal endoribonuclease domain, PF06479, 1104-1231 aa) and T. reesei Ire1 (1243 aa protein; XP_006962552.1, isolate QM6a; cd09769, 170-463 aa; IPR017441, 812-834 aa; PF06479, 1104-1231 aa) are shown. (B) Domain predictions for V. dahliae Hac1 ${ }^{\mathrm{u}}$ (526 aa protein; NLS 94-105 aa; bZIP 107-164 aa, PS50217) and A. nidulans HacA ${ }^{u}$ (441 aa protein; CBF87535.1; FGSC A4; NLS 55-65 aa; bZIP 87-150aa, PS50217) are shown.

\subsubsection{The HAC1 mRNA is unconventionally spliced in $V$. dahliae}

The Ensembl Fungi gene annotation of the potential HAC1 homolog in V. dahliae predicted only one mRNA splice variant with a $53 \mathrm{nt}$ intron, resulting in a spliced $1581 \mathrm{nt}$ mRNA. Since unconventional splicing of the HAC1 mRNA was described for known homologs preferentially under ER stress inducing conditions, it was tested, whether different mRNA splice variants could be obtained for $V$. dahliae HAC1 from liquid cultures in presence or absence of dithiothreitol (DTT). DTT is a reducing agent which prevents the formation of disulfide bonds and thereby induces misfolding of proteins in the ER (Guillemette et al., 2011).The HAC1 cDNA sequence amplified from un-induced growth was in accordance with the database predicted splice variant, referred to as $H A C 1^{u}$ (Figure 16A). Additionally, a second splice variant could be obtained from these growth conditions, which was also amplified from ER stress inducing growth conditions, named $H A C 1^{i}$ (Sequence given in S10). The HAC1' mRNA results from splicing of a second intron with $20 \mathrm{nt}$ in size (Figure 16A). Splicing of the $20 \mathrm{nt}$ intron alters the ORF, leading to a shorter variant of $1254 \mathrm{nt}$. 
HAC1 pre-mRNA $\overbrace{0290343}^{\sim 852} \underbrace{872}_{1634} \mathrm{nt}$

HAC1u
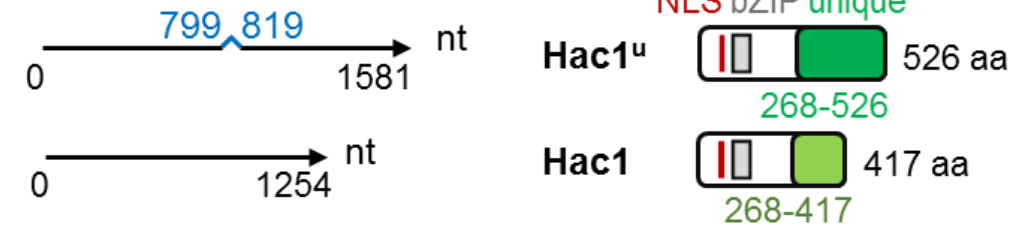

B

Consensus splice sites

V. dahliae

A. fumigatus

A. nidulans

T. reesei

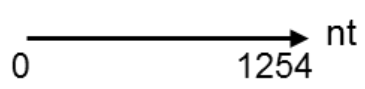

268-417

A. brassicicola

N. crassa

$U$. maydis

S. cerevisiae

5' CNGangn

H. sapiens

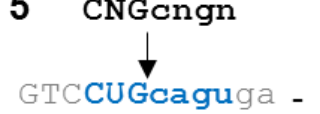

8 nt- gaccugCCGUGU

AUCCUGcagc $g g$ - 8 nt- gac cugCAGUGU

AUCCUGcagcga - 8 nt- gaccugCAGUGU

GUCCUGcagaga - 8 nt- gacccgCAGUGU

C

S. cerevisiae hac1

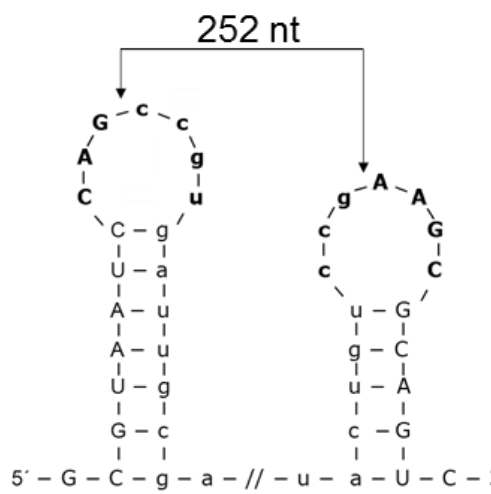

AUCCUGccgcga - 8 nt- gaccugCCGUGU

AUCCUGcugugg - 11 nt- gaCcugCAGUGU

UCCCUGcagc $g g$ - 53 nt- uCC CagCAGC $G \mathrm{G}$

AUCCAGccguga - 240 nt- uguccgAAGC $G C$

AGUCCGcagcac - 14 nt- $C$ CucugCAGC $A G$

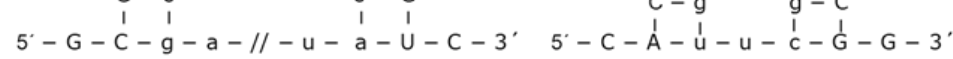

Figure 16: The V. dahliae HAC1 mRNA is unconventionally spliced. (A) mRNA and protein variants of $V$. dahliae HAC1. The $2634 \mathrm{nt} H A C 1$ pre-mRNA contains a $53 \mathrm{nt}$ conventional (black) and a 20 nt unconventional (blue) intron. V. dahliae produces two HAC1 mRNA variants: splicing of the conventional intron results in $H A C 1^{u}$ mRNA (1581 nt) for the potential $\mathrm{Hac}^{\mathrm{u}}$ protein (526 aa), additional unconventional splicing results in a shorter induced HAC1 $1^{\circ}$ ORF (1254 nt) encoding the Hac1 protein (417 aa). N-termini (268 aa) of both proteins are identical, containing a basic leucine zipper domain (bZIP, grey, PS50217; 107$164 \mathrm{aa}$ ) and a nuclear localization signal (NLS, red, 94-105 aa), whereas Hac1" ${ }^{\mathrm{u}}$ and Hac1 possess unique C-termini (dark or light green). (B) Alignment of the 5'- and $3^{\prime}$-splice sequences of the unconventionally spliced introns shows high conversation of $V$. dahliae HAC1 (VDAG_JR2_Chr2g09780a; blue), A. fumigatus hacA (XM_743634), A. nidulans hacA (AN9397), T. reesei QM6a hac1 (M419DRAFT_128619), A. brassicicola HacA (Joubert et al., 2011), N. crassa hac-1 (NCU01856), U. maydis cib1 (UMAG_11782), S. cerevisiae S288C hac1 (NC_001138.5), H. sapiens XBP1 (NM_005080.3). The consensus sequence of Ire1 splice sites (Hooks \& Griffiths-Jones, 2011) is indicated by CNG'CNGN. Ire1 splice sites are indicated by arrows, intron sequences as lowercase characters, splice sequences as capital letters. Numbers of nucleotides for those not shown are given. (C) Twin stem-loop secondary structures of $5^{\prime}$ - and $3^{\prime}$-splice sequences of $V$. dahliae and $S$. cerevisiae unconventional HAC1 introns. Ire1 splice sites are indicated by arrows, intron sequences as lowercase characters, splice sequences as capital letters, discontinuation of intron sequence as $/ /$. RNAfold was used for folding prediction (http://rna.tbi.univie.ac.at/cgi-bin/RNAfold.cgi). 
In yeast and filamentous ascomycetes, translation of the uninduced mRNA variant into Hac1 ${ }^{\text {u }}$ was described to be blocked (Chapman \& Walter, 1997; Rüegsegger et al., 2001; Saloheimo et al., 2003; Mulder et al., 2004; Joubert et al., 2011; Heimel, 2015). Differently, translation of the uninduced protein variant, acting as negative regulator of the UPR, was observed in higher eukaryotes (Yoshida et al., 2006, 2009). Hints to protein translation were given in the basidiomycete U. maydis (Heimel et al., 2013). The alternative splice variant $H A C 1^{i}$ encodes a 417 aa Hac1 protein (aa sequence shown in Figure S11) with a molecular weight of $44 \mathrm{kDa}$. For both hypothetical Hac1 protein variants the N-terminal 268 aa are identical with a nuclear localization signal (NLS) and bZIP domain. The bZIP protein domains are specifically found in transcription factors and are formed by a basic loop responsible for DNA binding and the hydrophobic leucine zipper required for homo- or heterodimerization of two DNA binding proteins (Hurst, 1995). The NLS proposes protein import into the nucleus. Due to the frame shift after unconventional splicing, the $\mathrm{C}$-terminus of the predicted $\mathrm{Hac} 1^{\mathrm{u}}$ and $\mathrm{Hac} 1$ proteins are unique with 258 unique amino acids in Hac1 ${ }^{\text {u }}$ versus 149 in Hac1.

The mRNA of HAC1 homologs from several fungal species up to humans was described to be cleaved by the cytosolic endoribonuclease domain of Ire1 by recognition of the conserved consensus splice sequence $5^{\prime}-\mathrm{CNG}^{\prime} \mathrm{CNGN}-3^{\prime}$ which is specific for unconventional splicing (Yoshida et al., 2001; Saloheimo et al., 2003; Mulder et al., 2004; Wimalasena et al., 2008; Richie et al., 2009; Cheon et al., 2011; Hooks \& Griffiths-Jones, 2011; Joubert et al., 2011; Heimel et al., 2013; Montenegro-Montero et al., 2015). Alignment of the 5 '- and 3 '-intron-exon-borders of both introns from V. dahliae HAC1 to the consensus sequence displayed conservation of the $20 \mathrm{nt}$ intron, which was found to be spliced under ER stress induced growth conditions (Figure 16B). The $53 \mathrm{nt}$ intron spliced in both, $H A C 1^{u}$ and $H A C 1^{i}$, did not match the consensus sequence.

Comparison of the 20 nt intron from $V$. dahliae HAC1 to homologous sequences in other organisms displays the same intron size in A. brassicicola, $A$. fumigatus, $A$. nidulans, and T. reesei. $N$. crassa $(23 \mathrm{nt})$ and human $(26 \mathrm{nt})$ homologs possess similar intron sizes. The unconventionally spliced intron of the homolog Cib1 in U. maydis shows intermediate length $(65 \mathrm{nt})$, whereas the yeast $S$. cerevisiae homolog is much longer with a size of $252 \mathrm{nt}$ (Figure 16B).

The mRNA of HAC1 homologs was described to form a twin stem-loop structure in several organisms, which is targeted by the endonuclease domain of Ire1 homologs (Yoshida et al., 2001; Saloheimo et al., 2003; Mulder et al., 2004; Wimalasena et al., 2008; Richie et al., 2009; Cheon et al., 2011; Hooks \& Griffiths-Jones, 2011; Joubert et al., 2011; Heimel et al., 2013; Montenegro-Montero et al., 2015). In order to allow predictions about the splice mechanism for $V$. dahliae HAC1, the secondary structure of 
the unconventionally spliced intron was analyzed. Figure 16C shows the twin stem-loop secondary structure of the $20 \mathrm{nt}$ intron from $\mathrm{V}$. dahliae HAC1 in comparison to the model organism S. cerevisiae, calculated by the RNAfold web server with the lowest free energy. The predicted Ire1 cleavage sites are located in the formed seven nucleotides loops.

Similarities between the $V$. dahliae Hac1 protein encoded by the unconventionally spliced mRNA $H A C 1^{i}$ to described UPR regulatory proteins in other fungi and human XBP1 (Xbox binding protein) were assessed (Figure 17). The highest similarity was found to T. reesei $\mathrm{HACl}$ with $55 \%$ aa sequence identity. The corresponding $A$. nidulans and $A$. fumigatus proteins display a higher amino acid similarity to the $V$. dahliae protein with $36 \%$ than the S. cerevisiae homolog with $28 \%$. The similarities to $U$. maydis cib1 and $H$. sapiens XBP1 was below $20 \%$ (Figure 17A). The proteins from V.dahliae and T. reesei cluster in one subclade in the phylogenetic tree (Figure 17B). Considered homologs from ascomycetes form one clade with lower amino acid similarities to the homologs of the basidiomycete $U$. maydis and the $H$. sapiens homolog XBP1.

In summary, V. dahliae HAC1 shows high similarity to homologs in related fungi. It encodes an unconventionally spliced intron with a proposed twin stem-loop structure, which is presumably recognized by the endoribonuclease Ire1 and displays similarities in the deduced protein sequence and protein domain predictions to UPR regulatory proteins from related fungi.

A

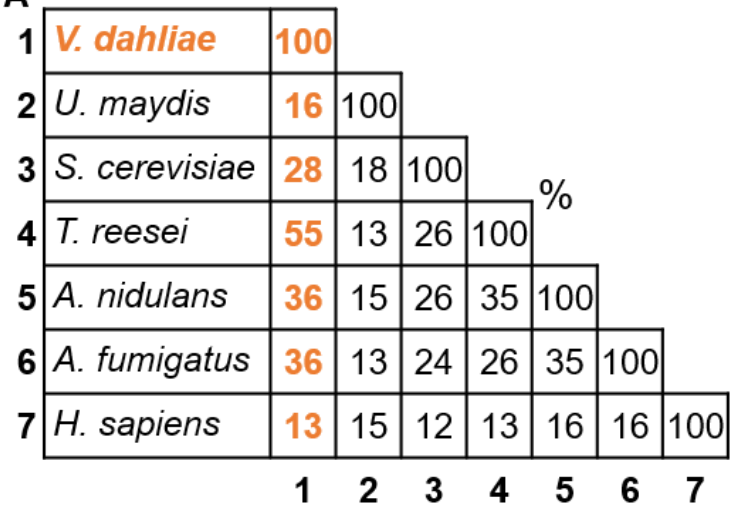

B

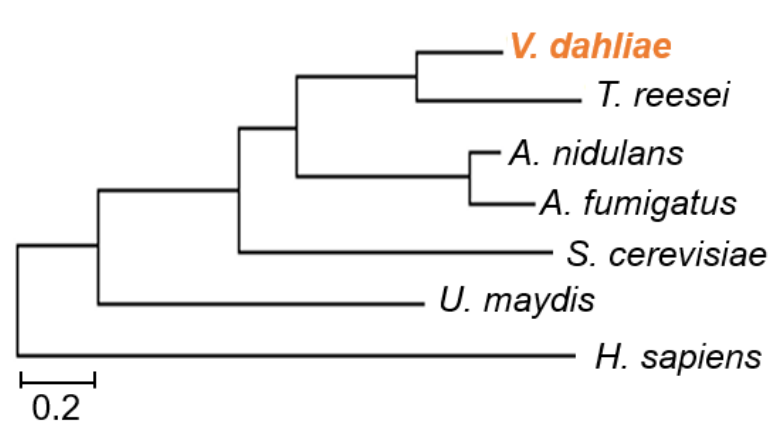

Figure 17: Similarities of Hac1 proteins from different organisms. (A) Protein sequence identity matrix of Hac1-like proteins (ClustalW algorithm, sequence identifiers given in $\mathbf{B}$ were used for the calculation). (B) Phylogenetic tree of Hac1-like proteins with V. dahliae Hac1 (Figure S11), U. maydis cib1s (XP_011390112.1; isolate 521), S. cerevisiae HAC1 ${ }^{\mathrm{i}}$ (NP_116622.1; isolate S288C), T. reesei HACli (XP_006964054.1; isolate QM6a), A. nidulans HacA' (Q8TFU8.2; isolate FGSC A4), A. fumigatus HacA' (ACJ61678.1; isolate H237), H. sapiens XBP1 (NP_001073007.1) sequences. ClustalW algorithm in MEGA6.0 was used with the Maximum likelihood method. The scale bar represents the average number of amino acid substitutions per site. 


\subsubsection{The unconventionally spliced mRNA variant $H A C 1^{i}$ is translated into the}

\section{Hac1 protein in V. dahliae}

In order to analyze the role of $H A C 1$ in the development and virulence of $V$. dahliae, a deletion strain was constructed. The impact of the different splice variants was tested by ectopic reintegration of the HAC1 gene or either one of the two mRNA splice variants fused to $H A$ at the $3^{\prime}$-end under control of the native promoter and terminator. Prior to functional investigation, correct integration of constructs was confirmed by Southern hybridization (Figure S8).

The expression levels of $H A C 1$ in the complementation strain $H A C 1-C$ and strains harboring the different splice variants, $H A C 1^{u}-H A$ and $H A C 1^{i}-H A$, were tested by quantitative reverse transcriptase PCR reactions in comparison to wild type and $\triangle H A C 1$ (Figure 18A).

A

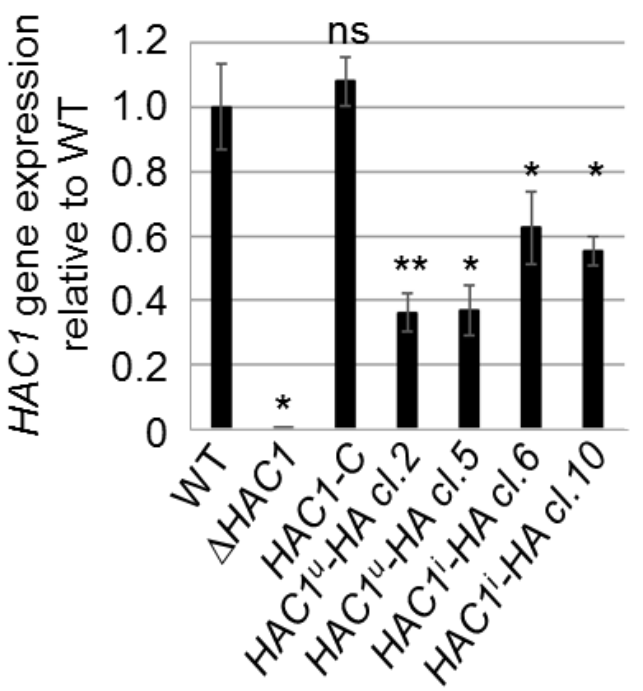

B

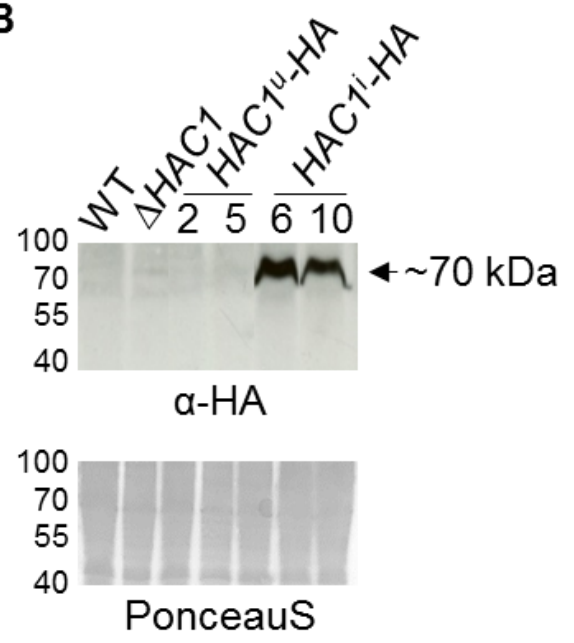

Figure 18: The V. dahliae unconventionally spliced mRNA HAC1i, but not the uninduced mRNA HAC1", is translated into the Hac1 protein. V. dahliae wild type JR2 (WT), HAC1 deletion ( $\triangle H A C 1$ - VGB371), complementation (HAC1-C - VGB382), as well as two transformants each of strains expressing ectopically integrated HAC1 mRNA splice variants fused to $H A$ at the $3^{\prime}$-end in the $\triangle H A C 1$ strain $\left(H A C 1^{4}-H A\right.$ transformant number 2 and 5 - VGB439, VGB440; HAC1'-HA transformant number 6 and 10 - VGB437, VGB438) were compared. (A) Quantification of HAC1 gene expression by primers targeting both HAC1 mRNA splice variants. $1 \times 10^{6}$ spores were inoculated in $50 \mathrm{ml} \mathrm{SXM}$ and incubated for three days at $25^{\circ} \mathrm{C}$ under constant agitation. HAC1 gene expression is decreased in $H A C 1^{u}-H A$ and $H A C 1^{i}-H A$. Mean values of two independent experiments with standard error of the mean normalized to wildtype and reference genes $H 2 A$ and EIF2B are shown. Significant differences to wild type calculated with one-way Anova and Student's t-test are shown with ${ }^{*} p<0.05 ;{ }^{* *} p<0.01$, ns $=$ non-significant. (B) Detection of Hac1 proteins in immunoblots using $\mathrm{HA}$-specific antibody and $80 \mu \mathrm{g}$ protein extracts isolated from mycelia grown in liquid PDM for four days at $25^{\circ} \mathrm{C}$ under constant agitation. PonceauS staining served as a loading control. A strong signal is only visible at $\sim 70 \mathrm{kDa}$ for tagged Hac1 in the constitutively spliced $H A C 1^{i}-H A$ strain instead of the predicted $46 \mathrm{kDA}$, whereas there was no specific band for the predicted Hac1 ${ }^{\mathrm{u}}-\mathrm{HA}$ protein $(59 \mathrm{kDa})$. 
The HAC1 expression was quantified for cultures grown in liquid simulated xylem medium for three days targeting both, induced and uninduced HAC1 mRNA variants, with the same primer pair. The HAC1-C complementation strain displayed wild type-like HAC1 expression levels (Figure 18A), whereas expression was reduced to less than $40 \%$ in $H A C 1^{u}-H A$ and to $~ 60 \%$ in $H A C 1^{i}-H A$ (Figure $18 \mathrm{~A}$ ).

Immunoblot analysis was performed to investigate whether both HAC1 mRNA variants were translated into $\mathrm{Hac}^{\mathrm{u}}{ }^{\mathrm{-}} \mathrm{HA}$ and $\mathrm{Hac1}-\mathrm{HA}$ fusion proteins with an $\mathrm{HA}$-antibody (Figure $18 \mathrm{~B})$. No bands were observed for $H A C 1^{u}-H A$ strains containing the conventionally spliced mRNA variant of HAC1 (Figure 18B). The predicted band size for the Hac1 ${ }^{\mathrm{u}}{ }_{-} \mathrm{HA}$ protein of $59 \mathrm{kDa}$ and the Hac1-HA protein of $46 \mathrm{kDa}$ were not detected, but a band with $\sim 70 \mathrm{kDa}$ was obtained for $H A C 1^{i}-H A$, expressing the unconventionally spliced $H A C 1$ mRNA variant (Figure 18B). These data suggest, that $H A C 1^{i}-H A$ is translated into a protein in $V$. dahliae, whereas the translation of $H A C 1^{u}-H A$ could not be observed.

\subsubsection{HAC1 is essential for microsclerotia formation of $V$. dahliae}

The impact of $V$. dahliae HAC1 on growth, microsclerotia formation, and fungal conidiation was investigated by comparison of the ex planta phenotypes of the $\triangle H A C 1$, $H A C 1-C, H A C 1^{u}-H A$ and $H A C 1^{i}-H A$ strains.

Vegetative growth of the $H A C 1$ deletion strain was severely impaired under all tested growth conditions (Figure 19). Colonies formed by the deletion strain ten days after spot inoculation formed less aerial mycelium, appeared less dense, and more transparent in comparison to wild type colonies grown on complete medium (PDM), minimal medium, or minimal medium supplemented with tunicamycin (Figure 19). Melanization of the $\triangle H A C 1$ colony centers was not observed under any tested growth condition. Furthermore, the deletion strain formed smaller colonies compared to wild type for every tested condition. Quantification of vegetative growth showed a significant decrease in the colony diameter about $12 \%$ for $\triangle H A C 1$ ten days after spot inoculation on CDM (Figure 19B).

Vegetative growth on minimal medium supplemented with tunicamycin was tested to examine the ability to cope with ER stress. Tunicamycin inhibits the glycosylation of proteins and, thereby, prevents folding in the ER und subsequent protein secretion (Guillemette et al., 2011). The mean colony diameter of $\triangle H A C 1$ grown on CDM supplemented with tunicamycin was $11 \%$ smaller than wild type colonies ten days after spot inoculation (Figure 19B). The supplementation of tunicamycin induced no additional decrease in the colony size of $\triangle H A C 1$ relative to wild type and the relative difference was unaltered (Figure 19B). 
A

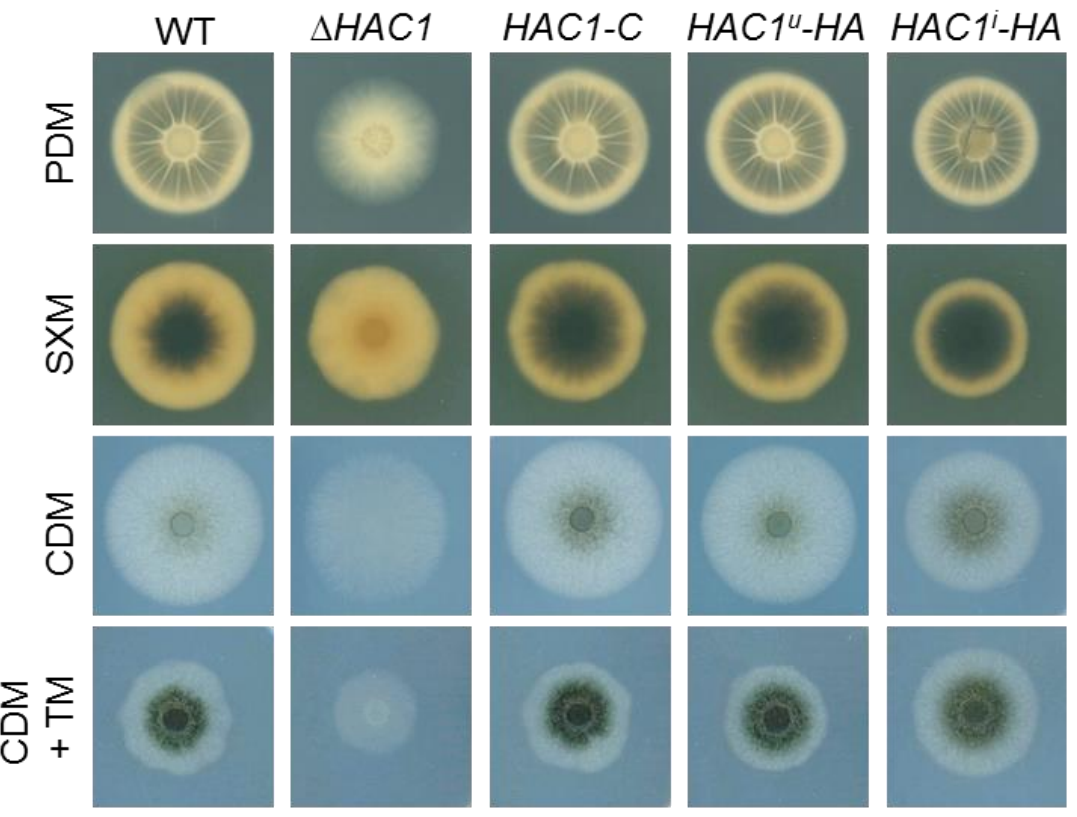

B

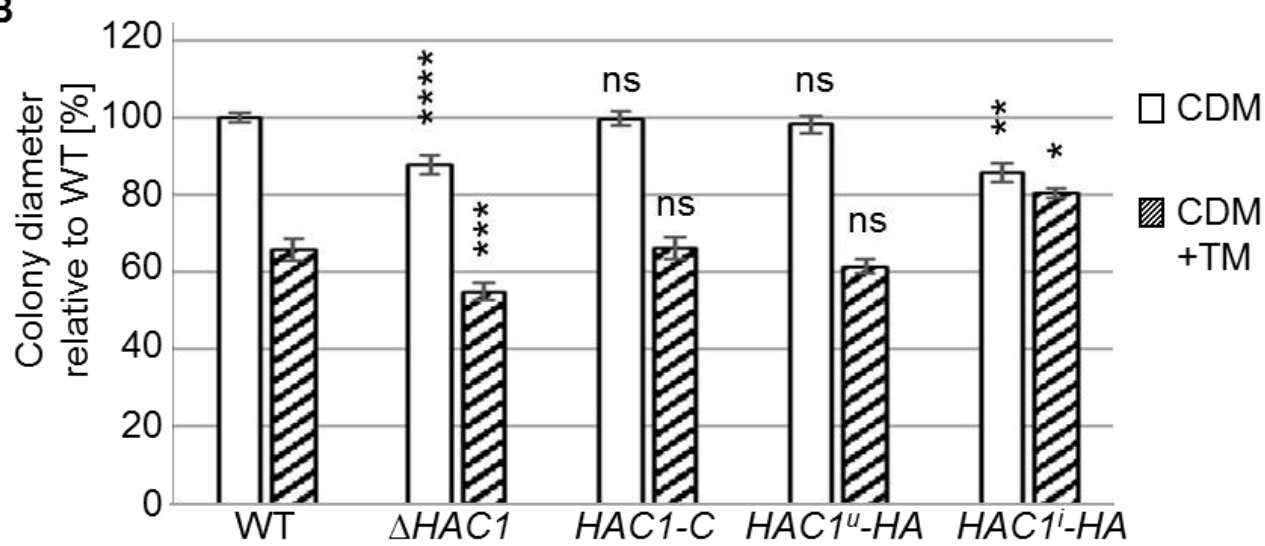

Figure 19: V. dahliae requires the expression of HAC1 for growth with or without tunicamycin-induced ER stress and melanization. Spot inoculation of 50000 spores from the wild type JR2 (WT) or $\triangle H A C 1, H A C 1-C, H A C 1^{u}-H A$, and $H A C 1^{i}-H A$ strains and incubation at $25^{\circ} \mathrm{C}$ for ten days. $\triangle H A C 1$ displays reduced vegetative growth compared to wild type. HAC1-C and HAC1u-HA strains display wild type-like growth, whereas colony diameters of $H A C 1^{i}-H A$ are decreased under non-stress conditions and relatively increased upon supplementation of tunicamycin. (A) Ex planta phenotypes on PDM, SXM, CDM, and CDM supplemented with $1 \mu \mathrm{g} / \mathrm{ml}$ tunicamycin (TM). $\triangle H A C 1$ forms less aerial mycelium, appears less dense, and more transparent on PDM, CDM, and CDM+TM. $\triangle H A C 1$ displays no melanization in the colony centers. (B) Quantification of vegetative growth. Growth was quantified by measurement of two perpendicular colony diameters for three colonies per transformant and medium $(\mathrm{n}=1)$. Two independent transformants were tested for $\triangle H A C 1$ (VGB371, VGB372), HAC14-HA (VGB439, VGB440), and HAC1'-HA (VGB437, VGB438), as well as one HAC1-C (VGB382) transformant. Bars represent mean values with standard deviations from three independent experiments for wild type and $\triangle H A C 1$, and two independent experiment for HAC1-C, HAC1 ${ }^{-}-H A$ and $H A C 1^{i}-H A$ grown on CDM. Mean values with standard deviations are shown from three independent experiments for wild type, $\triangle H A C 1$, and $H A C 1-C$, and two independent experiments for $H A C 1^{4}-H A$ and $H A C 1^{i}-H A$ grown on CDM+TM. Significant differences to wild type determined with one-way Anova and Student's t-test are shown with ${ }^{*} \mathrm{p}<0.05 ;{ }^{* *} \mathrm{p}<0.01 ;{ }^{* \star *} \mathrm{p}<0.001,{ }^{* * *} \mathrm{p}=0$, ns $=$ non-significant.

The HAC1-C and HAC14-HA strains displayed wild type-like growth. Colony diameters of $H A C 1^{i}-H A$ were decreased on all non-stress inducing media, but increased on CDM 
supplemented with tunicamycin (Figure 19). A decrease about $14 \%$ in the colony diameter was determined for the HAC1 ${ }^{i}-H A$ strain relative to wild type on CDM, whereas supplementation of tunicamycin resulted in a $\sim 15 \%$ increase of the colony diameters relative to wild type (Figure 19B). This observations suggests that the presence of the unconventionally spliced $H A C 1$ mRNA in the $H A C 1^{i}-H A$ strain and constitutive activation of the UPR enable a more efficient response to the induced ER stress.

Comparison of the ex planta phenotypes of the HAC1 deletion and the HAC1-C, $H A C 1^{u}-H A$, and $H A C 1^{i}-H A$ strains to wild type revealed the absence of melanized colony centers for $\triangle H A C 1$ on all tested media, whereas the HAC1-C and HAC1 ${ }^{-} H A$ strains displayed increased melanization. The ability of $\triangle H A C 1$ to develop microsclerotia as resting structures, maybe in an unmelanized form, was further analyzed in comparison to the complementation strains. The differences in melanization are most apparent in the colony centers and in colony cross sections (Figure 20). During microscopy of fungal material from colony centers of $\triangle H A C 1$ no melanized or unmelanized microsclerotia were observed for any medium, as exemplified for CDM in Figure 20.

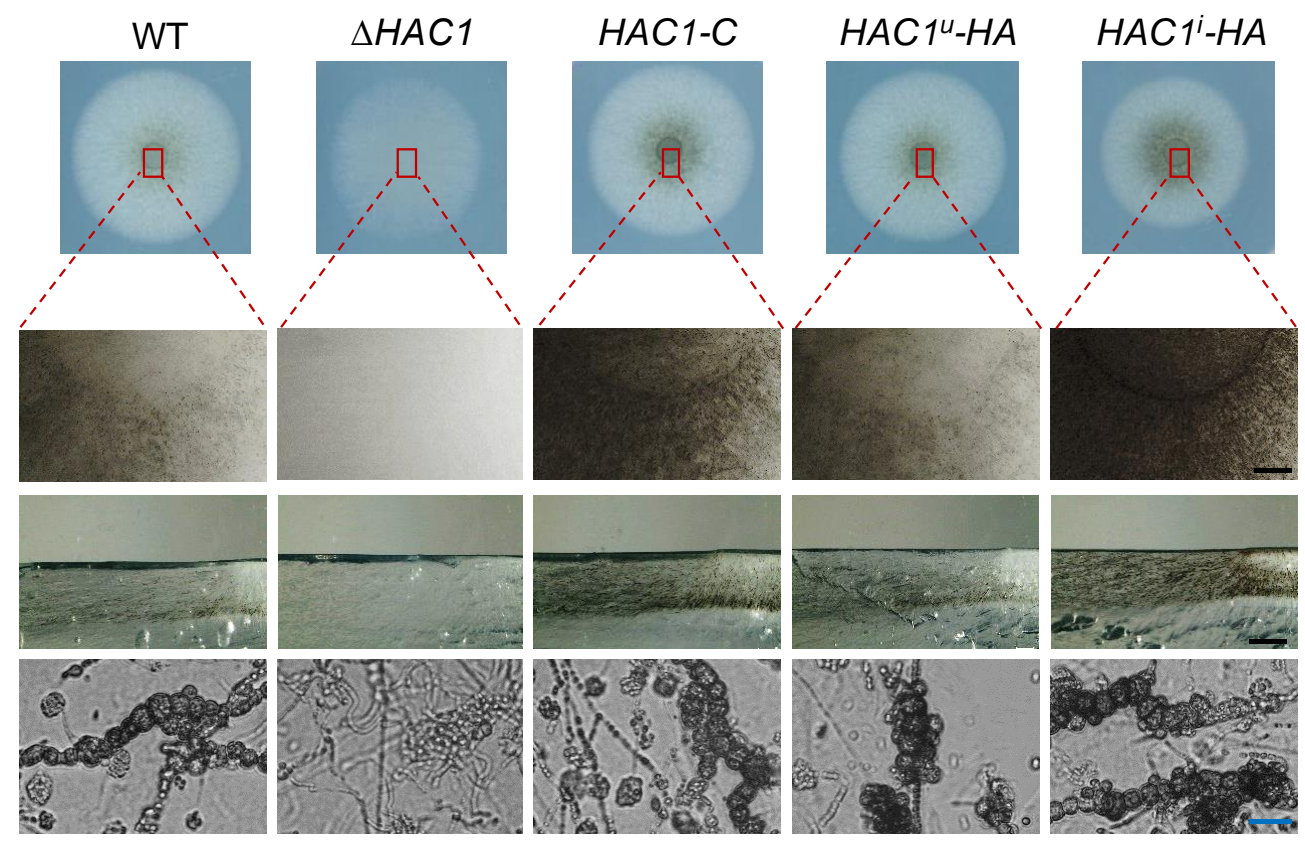

Figure 20: V. dahliae HAC1 is essential for microsclerotia formation. Microsclerotia formation was examined in $\triangle H A C 1, H A C 1-C, H A C 1^{4}-H A$, and $H A C 1^{i}-H A$ strains by spot inoculation of 50000 spores on $\mathrm{CDM}$ and incubation at $25{ }^{\circ} \mathrm{C}$ for ten days. Two independent transformants were tested for $\triangle H A C 1$ (VGB371, VGB372), HAC14-HA (VGB439, VGB440), and HAC1'-HA (VGB437, VGB438) strains and a single transformant for the HAC1-C (VGB382) strain. Close up pictures were taken from colony centers (indicated as red boxes) and cross sections were cut to visualize the appearance of microsclerotia as melanized structures in the agar. Fungal material from colony centers was scraped off for microscopy to observe microsclerotia morphology. Neither melanized nor unmelanized microsclerotia were observed for the $\triangle H A C 1$ strain, whereas formation of wild type-like microsclerotia was observed for all other strains. Microsclerotia formation was qualitatively increased in the $H A C 1-C$ and the $H A C 1^{i}-H A$ strains in comparison to wild type. Black scale bar $=1 \mathrm{~mm}$, blue scale bar $=20 \mu \mathrm{m}$. 
For all complementation strains, wild type-like shaped and melanized microsclerotia could be observed, however, in qualitatively increased occurrence for the HAC1-C and $H A C 1^{i}-H A$ strains and wild type-like frequencies in the $H A C 1^{u}-H A$ strain (Figure 20). The absence of microsclerotia in $\triangle H A C 1$ and the increase in melanization in $H A C 1^{i}-H A$ regardless of the decreased $H A C 1$ expression levels in $H A C 1^{i}-H A$ (Figure 18A) corroborate the role of $H A C 1$ in regulation of microsclerotia formation.

Within the plants vascular system $V$. dahliae forms conidiospores for spreading and systemic colonization of the host. The role of the UPR regulator Hac1 in conidiation was characterized by examination of the ability to form spores in the HAC1 deletion strain compared to wild type and the HAC1-C complementation strain. Therefore, the same number of spores was inoculated in liquid simulated xylem medium and quantified after five days. The HAC1 deletion strain displayed significantly reduced conidiospore numbers to $15 \%$ of the wild type level (Figure 21). Conidia levels of the HAC1-C strain were more similar to wild type with $86 \%$.

In summary, $H A C 1$ is essential for microsclerotia formation and has important impacts on conidiation. Constant activation of the UPR in the HAC1'-HA strain is correlated with increased growth in response to ER stress and increased microsclerotia formation in V. dahliae.

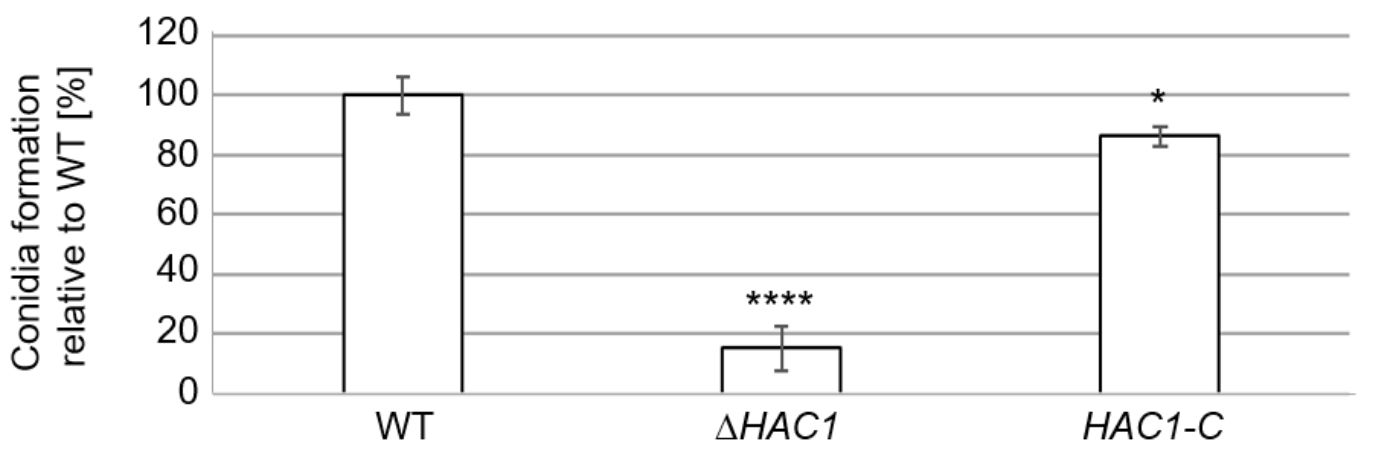

Figure 21: V. dahliae HAC1 positively regulates conidiospore formation. Quantification of conidiation was performed by inoculation of $4000 \mathrm{spores} / \mathrm{ml}$ in liquid SXM and incubation at $25^{\circ} \mathrm{C}$ under constant agitation for five days in triplicates. The number of spores was determined for the wild type JR2, two independent transformants of $\triangle H A C 1$ (VGB371, VGB372), and the HAC1-C (VGB382) complementation strain in three independent experiments. The numbers of conidia relative to wild type are shown. The values determined for two independent $\triangle H A C 1$ transformants are represented in one bar. Significant differences to wild type determined by one-way Anova and Student's t-test are shown with ${ }^{* * *} \mathrm{p}=0$, ${ }^{*} \mathrm{p}<0.05$ with $\mathrm{n}(\mathrm{WT})=3, \mathrm{n}(\triangle H A C 1)=6, \mathrm{n}(H A C 1-C)=3$. The HAC1 deletion strain shows reduced conidia formation. Conidia formation of $H A C 1-C$ was more similar to the wild typelevel. 


\subsubsection{V. dahliae HAC1 is dispensable for penetration of the A. thaliana root cortex}

A functional UPR was described to be essential for fungal propagation after penetration of plant cells in U. maydis. In both, U. maydis and A. brassicicola, the UPR regulatory transcription factor is essential for induction of disease symptoms in plants. Different to these two plant pathogenic species, which infect plants via the leaf surface by formation of appressoria, initial colonization of the host plant by $V$. dahliae occurs via the roots by intercellular growth or development of hyphopodia (Reusche et al., 2014; Zhao et al., $2014,2016)$. So far, the role of the UPR in initial colonization of the host and virulence was not described for root colonizing plant pathogens. Here, the root colonization behavior of the UPR defective V. dahliae HAC1 deletion strain was examined (Figure 22).
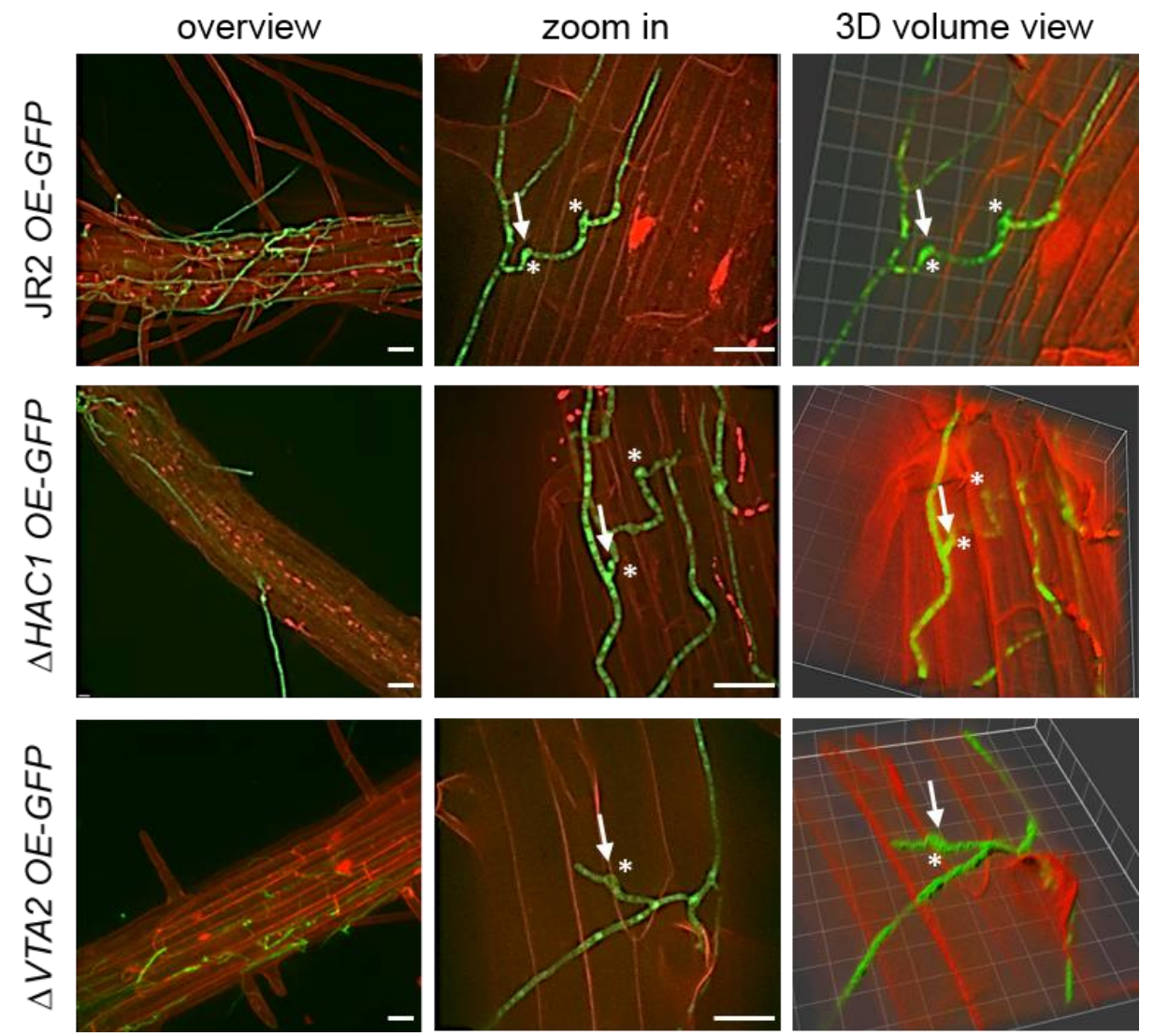

Figure 22: V. dahliae HAC1 is involved in initial colonization but dispensable for penetration of $\boldsymbol{A}$. thaliana roots. Fluorescence microscopy pictures of three-week-old A. thaliana plants seven days post inoculation with the same numbers of spores from wild type JR2, $\triangle H A C 1$, and $\triangle V T A 2$ constitutively expressing ectopic GFP. Overview pictures (left) as well as close up pictures (middle) were taken. 3D volume view pictures (right) calculated as maximum intensity projections of a Z-stack of microscope images are displayed. Four plants inoculated with either JR2 OE-GFP (VGB392) or $\triangle H A C 1$ OE-GFP (VGB380) strains in two independent experiments and two plants inoculated with spores obtained from $\triangle V T A 2$ $O E$-GFP strain were tested. Root cells were stained with $0.05 \%$ propidium iodide $/ 0.01 \%$ silwet solution. $\triangle H A C 1$ OE-GFP displays reduced propagation on the root surface, but is able to penetrate $A$. thaliana roots and propagate after invasion of the outer layer. Scale bars $=20 \mu \mathrm{m}$, white arrows = root penetration sites, asterisks = changes of growth direction . 
A HAC1 deletion strain expressing ectopically integrated GFP under control of the gpdA promoter was constructed in order to investigate the root colonization behavior in absence of HAC1. The $\triangle H A C 1$ OE-GFP strain was confirmed by fluorescence microscopy and Southern hybridization analysis (Figure S8). Three-week-old $A$. thaliana plants were inoculated with the same number of spores obtained from $\triangle H A C 1$ OE-GFP as well as from the wild type expressing free GFP and the fungal colonization behavior was observed after five to seven days. The Verticillium transcription activator of adhesion (VTA2) deletion strain served as control. $\triangle$ VTA2 penetrates plant roots like the wild type with less efficient proliferation inside the plant (Tran et al., 2014).

Overall less hyphae of the $\triangle H A C 1$ OE-GFP strain were observed on the root surface in comparison to wild type and the $\triangle V T A 2$ control strain five to seven days after root dipping (Figure 22). Penetration sites, recognizable by the formation of swollen hyphal tips and change in the growth direction, were observed in absence of HAC1 similar to wild type and the $\triangle V T A 2$ strain, even if more frequently in the wild type (Figure 22). Furthermore, hyphal growth after invasion of the outer layer was observed for the $\triangle H A C 1$ OE-GFP strain, as shown in the 3D volume view picture in Figure 22.

Therefore, $V$. dahliae HAC1 is involved in initial colonization of the outer root surface, but is neither required for penetration of the $A$. thaliana root nor for invasion of the outer root cortex.

\subsubsection{V. dahliae HAC1 is required for colonization of tomato plants}

A. thaliana root colonization assays had shown that $V$. dahliae HAC1 is involved in colonization of the root surface, but is dispensable for penetration and initial propagation within the root cortex. To investigate the role of HAC1 in subsequent steps of plant colonization and induction of disease symptoms, the HAC1 deletion strain as well as $H A C 1-C$ and the $H A C 1^{u}-H A$ and $H A C 1^{i}-H A$ strains were tested in tomato infection experiments (Figure 23).

$\triangle H A C 1$ induced significantly less severe disease symptoms than the wild type 21 days after spore inoculation with about $70 \%$ of plants displaying no disease symptoms. None of the $\triangle H A C 1$ treated plants displayed heavy symptoms and no hypocotyl discolorations were observed for any of these plants. The in planta phenotype of the $\triangle H A C 1$ strain was partially complemented in $H A C 1-C, H A C 1^{u}-H A$ and $H A C 1^{i}-H A$ treated plants. All of these plants displayed discoloration of the hypocotyl comparable to wild type infected plants (Figure 23A). HAC1-C treated plants showed only a slight reduction in disease symptoms, whereas inoculation with the $H A C 1^{u}-H A$ or $H A C 1^{i}-H A$ strains caused similar and even less severe symptoms in tomato plants in comparison to wild type. 
A
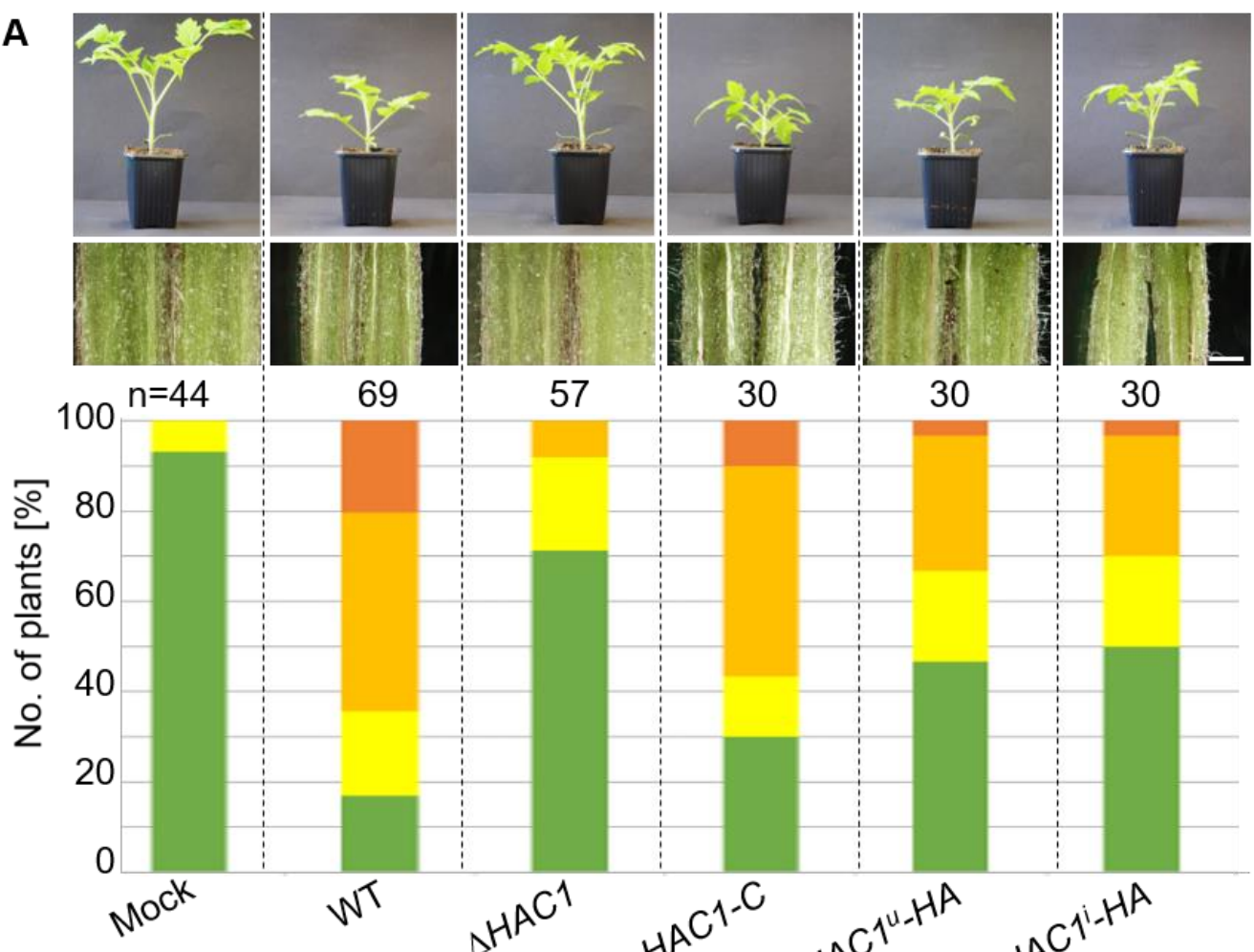

69
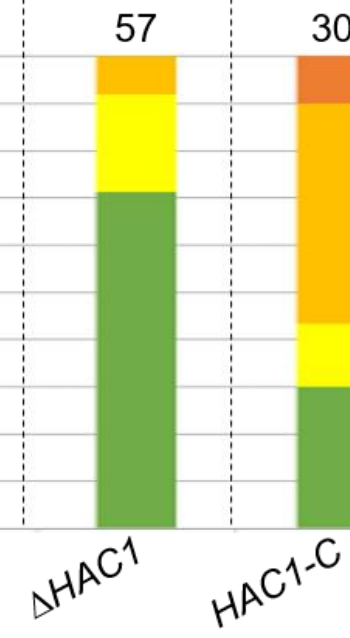

$30 \quad 30$

30

Disease scores:

$\square$ Healthy plant $\square$ Weak symptoms $\square$ Strong symptoms $\square$ Heavy symptoms

B

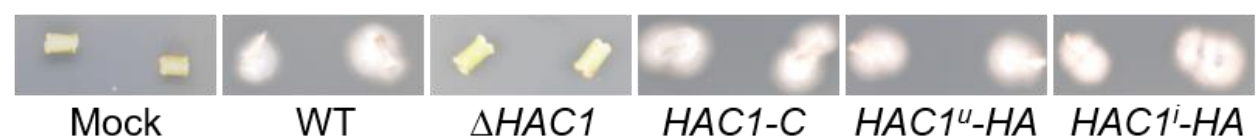

Figure 23: $V$. dahliae $H A C 1$ is required for induction of disease symptoms in S. Iycopersicum. (A) Ten-day-old tomato plants were inoculated by root dipping into water control (mock) or spores obtained from wild type JR2 (WT), HAC1 deletion, complementation strain $(H A C 1-C)$, or from strains ectopically expressing either the uninduced $\left(H A C 1^{u}-H A\right)$ or the induced $\left(H A C 1^{i}-H A\right)$ mRNA variant under control of the native promoter and terminator. Representative plants and hypocotyl dissections 21 days post inoculation are shown. The relative number of plants with certain disease scores are displayed in the stack diagram. Two independent transformants of $\triangle H A C 1$ (VGB371, VGB372) were tested in three independent experiments. One transformant of HAC1-C (VGB382) was tested in two independent experiments. Two independent transformants of HAC14-HA (VGB439, VGB440) and HAC1'-HA (VGB437, VGB438) were tested in one experiment. Only $\triangle H A C 1$ induced less severe disease symptoms in comparison to wild type. Different to plants treated with all other tested strains, the hypocotyl of $\triangle H A C 1$ inoculated plants appears mock-like (Scale bar $=1 \mathrm{~mm}$ ). (B) Fungal outgrowth from stem sections. Stems were harvested, surface sterilized, cut, and incubated on PDM supplemented with chloramphenicol for seven days at $25^{\circ} \mathrm{C}$. $\triangle H A C 1$ could not be re-isolated from surface sterilized stem sections.Stems from plants infected with all other tested strains displayed fungal outgrowth after seven days.

Fungal outgrowth from treated plants was tested to investigate whether the strains, especially $\triangle H A C 1$, were able to invade plant tissues. Therefore, stems from tomato plants used for determination of disease scores 21 days after inoculation were surfacesterilized, cut into stem sections, and incubated on PDM agar supplemented with 
chloramphenicol for seven days at $25^{\circ} \mathrm{C}$. Fungal outgrowth was observed for all strains except $\triangle H A C 1$ (Figure 23B).

Hence, the absence of HAC1 caused severely decreased development of disease symptoms in tomato plants and did not allow its re-isolation from infected tissue suggesting that $H A C 1$ is required to survive within the plants vascular system. Additionally, in an experiment with one repetition with two independent transformants each, a constitutively active UPR in $H A C 1^{i}-H A$ led to the induction of unaltered disease symptoms in tomato plants in comparison to the strain $H A C 1^{u}-H A$ harboring the uninduced mRNA splice variant of $H A C 1$ with intermediate disease severity. This result suggests that virulence of $V$. dahliae is unaltered in presence of a constitutively active UPR.

\subsection{The $V$. dahliae oleate $\Delta 12$-fatty acid desaturase Ode1 promotes differentiation with only a minor impact on virulence}

\subsubsection{V. dahliae Ode1 corresponds to the A. nidulans oleate $\Delta 12$-fatty acid desaturase OdeA}

Besides the UPR-monitored secretion of proteins, lipid molecules are involved in hostfungus communication. Linoleic acid and the derived oxylipins from plant and fungus can modulate fungal differentiation and the development of disease symptoms (Calvo et al., 2001; Brodhagen et al., 2008; Brodhun et al., 2009; Reverberi et al., 2010; Scala et al., 2014; Fischer \& Keller, 2016; Patkar \& Naqvi, 2017). Oleate $\Delta 12$-fatty acid desaturases catalyze the oxidation of oleic acid to linoleic acid by introduction of a second double bond at position 12 of the carbon chain (Los \& Murata, 1998). The impact of the oleate $\Delta 12$-fatty acid desaturase OdeA on fungal differentiation was described in two Aspergilli (Calvo et al., 2001; Chang et al., 2004; Wilson et al., 2004), however, it was not characterized in plant pathogens. The impact of the corresponding desaturase Ode1 in $V$. dahliae on differentiation and virulence was analyzed.

V. dahliae VDAG_JR2_Chr1g29610a was identified as the A. nidulans odeA (AAG36933.1) homolog by reciprocal BLAST search of the deduced amino acid sequences against the $V$. dahliae JR2 (Ensembl Fungi) and the A. nidulans (NCBI database) proteome with $66 \%$ aa sequence identity. The unspliced $V$. dahliae ODE1 mRNA of $1504 \mathrm{bp}$ contains two exons. The predicted intron-exon structure was confirmed by PCR amplification of the spliced transcript from wild type cDNA (Figure 24). The deduced Ode1 protein with a length of 481 aa and a predicted molecular weight of $54 \mathrm{kDa}$ contains two fatty acid desaturase (FAD) domains. The cytosolic catalytic center of $\Delta 12$-fatty acid desaturases is generally described to be formed by conserved $\mathrm{N}$ - and C-terminal histidine clusters described as FAD domains and iron atoms provided from 
the membrane-bound donor cytochrome $b_{5}$ (Los \& Murata, 1998). The $V$. dahliae Ode1 protein was predicted as a transmembrane protein with four putative hydrophobic transmembrane helices, two short non-cytosolic regions, and three hydrophile cytosolic regions, including the $\mathrm{N}$ - and $\mathrm{C}$-termini of the protein (Figure 24). The catalytic center of the desaturase was proposed to be directed to the cytosol. Ode1 was suggested to be localized to plastid membranes with $89 \%$ and in the ER membrane with $7 \%$ probability as the second-best hit by the DeepLoc-1.0 prediction tool. Since fungi do not possess plastids, the localization in the ER is the most likely suggestion from this database. For other fungal $\Delta 12$ - and $\Delta 15$-fatty acid desaturases, localization to the ER was described (Martin et al., 2007; Gostinčar et al., 2009).

A

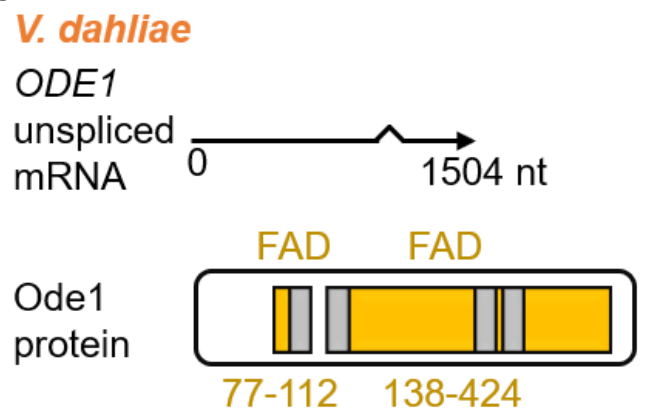

B 481 aa

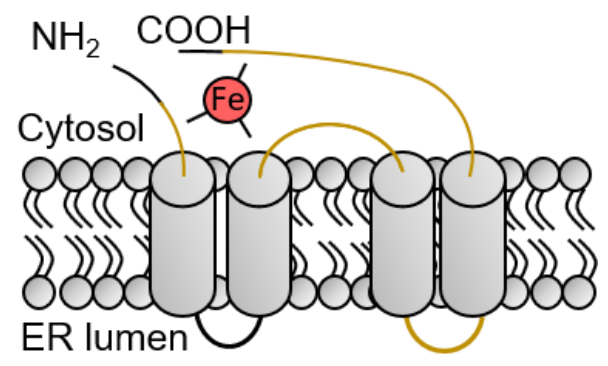

Figure 24: $V$. dahliae ODE1 encodes a membrane-bound oleate $\Delta 12$-fatty acid desaturase. (A) The $V$. dahliae oleate $\Delta 12$-fatty acid desaturase Ode1 is encoded by the gene VDAG_JR2_Chr1g29610a. The intron-exon structure of the 1504 bp ORF predicted by Ensembl Fungi was confirmed by PCR amplification of the spliced transcript from wild type cDNA. The deduced Ode1 protein consists of 481 aa. The protein contains two fatty acid desaturase domains (FAD; yellow; 77-112 aa, IPR021863; 138-424 aa; IPR005804) and four putative transmembrane helices (grey; 105-124 aa, 136-157 aa, 300-319 aa, 331-350 aa; Phobius). Domains were predicted by InterProScan. (B) Scheme of the predicted Ode1 protein domain localization based on the InterProScan and DeepLoc-1.0 prediction tools. The $\mathrm{N}$ - and C-termini of the protein are directed to the cytosol. An iron atom and the FAD domains form the catalytic center of Ode1. Four transmembrane protein domains were proposed. Yellow $=$ FAD domains, $\mathrm{Fe}=$ iron atom (based on Los \& Murata, 1998).

\subsubsection{V. dahliae Ode1 localizes to plasma membranes and cell organelle membranes}

In order to analyze the role of Ode1 and Ode1-derived products in development and virulence of $V$. dahliae an ODE1 deletion strain was constructed. Strains harboring ODE1 fused to GFP either at the $5^{\prime}$ - or $3^{\prime}$-end at the endogenous locus under control of the native promoter and terminator were constructed to analyze the protein localization (Figure 25) and used as complementation strains. Prior to functional investigation, correct integration of deletion and GFP-labeled constructs was confirmed by Southern hybridization (Figure S9). 
A

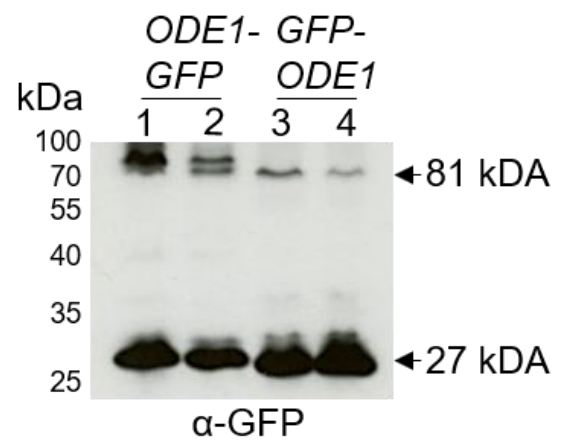

C
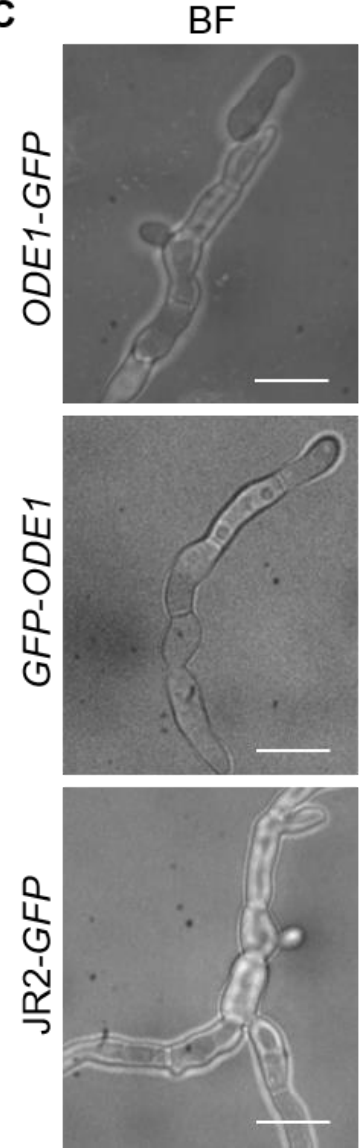

B
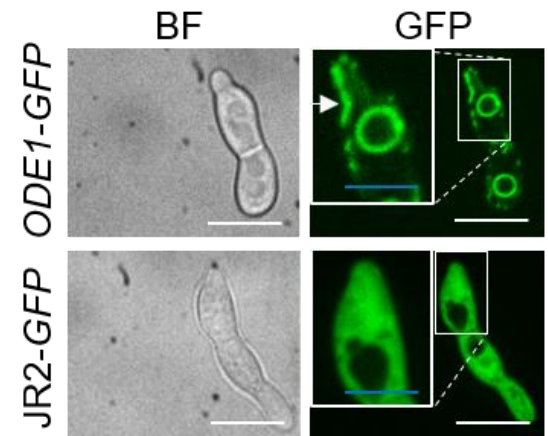

FM4-64
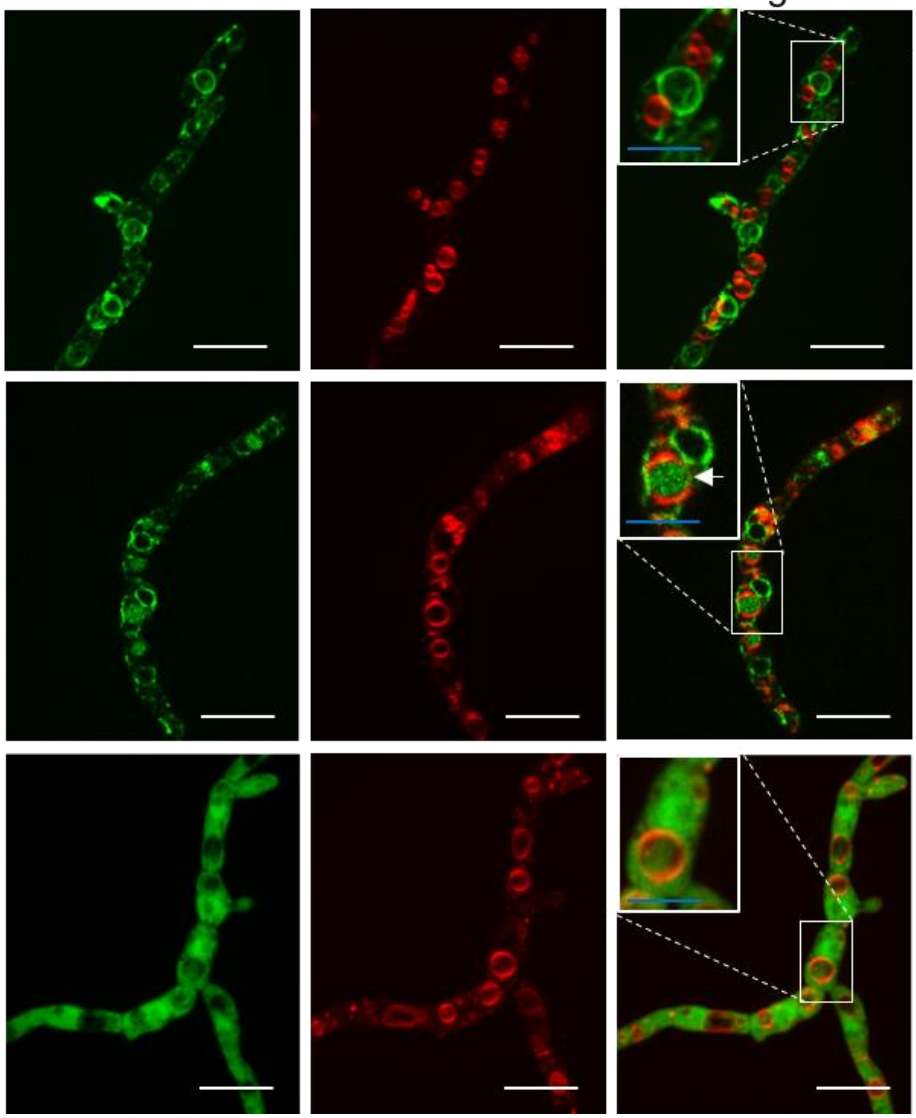

Figure 25: V. dahliae Ode1 is localized to plasma and organelle membranes different to vacuoles. (A) Immunoblot of complementation strains harboring ODE1-GFP or GFP-ODE1 at the endogenous locus under control of the native promoter and terminator. $80 \mu \mathrm{g}$ protein extracts isolated from ODE1-GFP transformant number 1 (VGB358) and 2 (VGB359) or GFP-ODE1 transformant number 3 (VGB360) and 4 (VGB361) grown in liquid PDM for four days at $25^{\circ} \mathrm{C}$ and a GFP-specific antibody were used. Ode1-GFP (81 kDa) and free GFP $(27 \mathrm{kDa})$ were detected, as well as an additional band for ODE1-GFP transformants. (B) Subcellular localization of Ode1-GFP after 12 hours at $25^{\circ} \mathrm{C}$ in liquid PDM. ODE1-GFP (VGB358) and wild type JR2 expressing ectopically integrated GFP under control of a constitutively active promotor were observed by fluorescence microscopy. Ode1-GFP localized to membranes of round cell organelles and close to hyphal tips of growing hyphae (indicated by white arrow). (C) Subcellular localization of Ode1-GFP and GFP-Ode1. Fluorescence microscopy 16 hours post inoculation of fungal spores (VGB358361 ) in liquid PDM and incubation at $25^{\circ} \mathrm{C}$ in comparison to free GFP in the JR2-GFP control strain. Vacuoles were stained in red with FM4-64. Ode1 localized to membranes of round cell organelles different than vacuoles. For GFP-ODE1, diffuse GFP signals were observed inside vacuoles as well (indicated by white arrows). Blue scale bars $=20 \mu \mathrm{m}$, white scale bars $=10 \mu \mathrm{m}$. 
The presence of the fusion proteins in fungal cells was verified for two independent transformants per strain in immunoblots (Figure 25A). Ode1 C-terminally fused to GFP displayed two bands, one with the predicted molecular weight of $81 \mathrm{kDa}$ and a second between $\sim 90-100 \mathrm{kDa}$, whereas only the band at $81 \mathrm{kDa}$ was observed for N-terminally tagged Ode1 (Figure 25A). In both, GFP-ODE1 and ODE1-GFP strains, the band representing free GFP at $\sim 27 \mathrm{kDa}$ was relatively strong in protein extracts isolated from mycelium grown for four days in complete medium (PDM), but more prominent for the strains harboring Ode1 N-terminally fused to GFP (Figure 25A).

For the protein sequence of the $V$. dahliae desaturase Ode1 four transmembrane helices were predicted (Figure 24). The subcellular localization of $\mathrm{N}$ - or C-terminal GFP fusion proteins under control of the native promoter was studied in the ODE1-GFP and GFP-ODE1 complementation strains in comparison to the wild type JR2 expressing ectopically integrated GFP under control of the constitutively active gpdA promotor. The GFP signals were observed by fluorescence microscopy 12 and 16 hours post inoculation of fungal spores in liquid complete medium (Figure 25B, C).

The GFP-signals were localized to membranes of cell organelles and to a minor extend to plasma membranes in fungal hyphae of both, ODE1-GFP and GFP-ODE1 strains (Figure 25B, C). Further, a localization to plasma membranes close to tips of growing hyphae was observed frequently (Figure 25B). Localization of Ode1 in the membranes of vacuoles could be excluded by FM4-64 staining (Figure 25C). Additionally, the presence of a diffuse GFP signal within FM4-64 stained vacuoles was observed in the strain producing Ode1 proteins $\mathrm{N}$-terminally fused to GFP. This observation suggests GFP-Ode1 to be less stable, resulting in the presence of free GFP and fusion protein degradation products in the vacuoles (Figure 25C).

\subsubsection{ODE1 is required for vegetative growth and microsclerotia formation in V. dahliae}

Oleate $\Delta 12$-fatty acid desaturases produce linoleic acid as an important membrane component. Membrane fluidity usually increases correlated with an increasing content of unsaturated fatty acids (Mansilla et al., 2004). Linoleic acid and the derived products are the major precursors for oxylipins with different impacts on fungal differentiation (Champe \& El-Zayat, 1989; Brodhun \& Feussner, 2011). The role of Ode1 in development of $V$. dahliae was examined testing vegetative growth and formation of microsclerotia as resting structures of the $O D E 1$ deletion strain under physiological and membrane stress inducing conditions in comparison to wild type and complementation strains.

Vegetative growth of the $\triangle O D E 1$ strain was tested by spot inoculation on various media including complete medium, simulated xylem medium, minimal medium with different 
carbon sources (sucrose, galactose, glucose, or cellulose), and on media supplemented with stress inducing agents $\left(\mathrm{NaCl}, \mathrm{H}_{2} \mathrm{O}_{2}\right.$, SDS) to investigate, whether ODE1 is important for vegetative growth or to deal with membrane stress in $V$. dahliae. Vegetative growth of the $\triangle O D E 1$ strain was decreased in all tested conditions. Examples are shown in Figure 26.

A

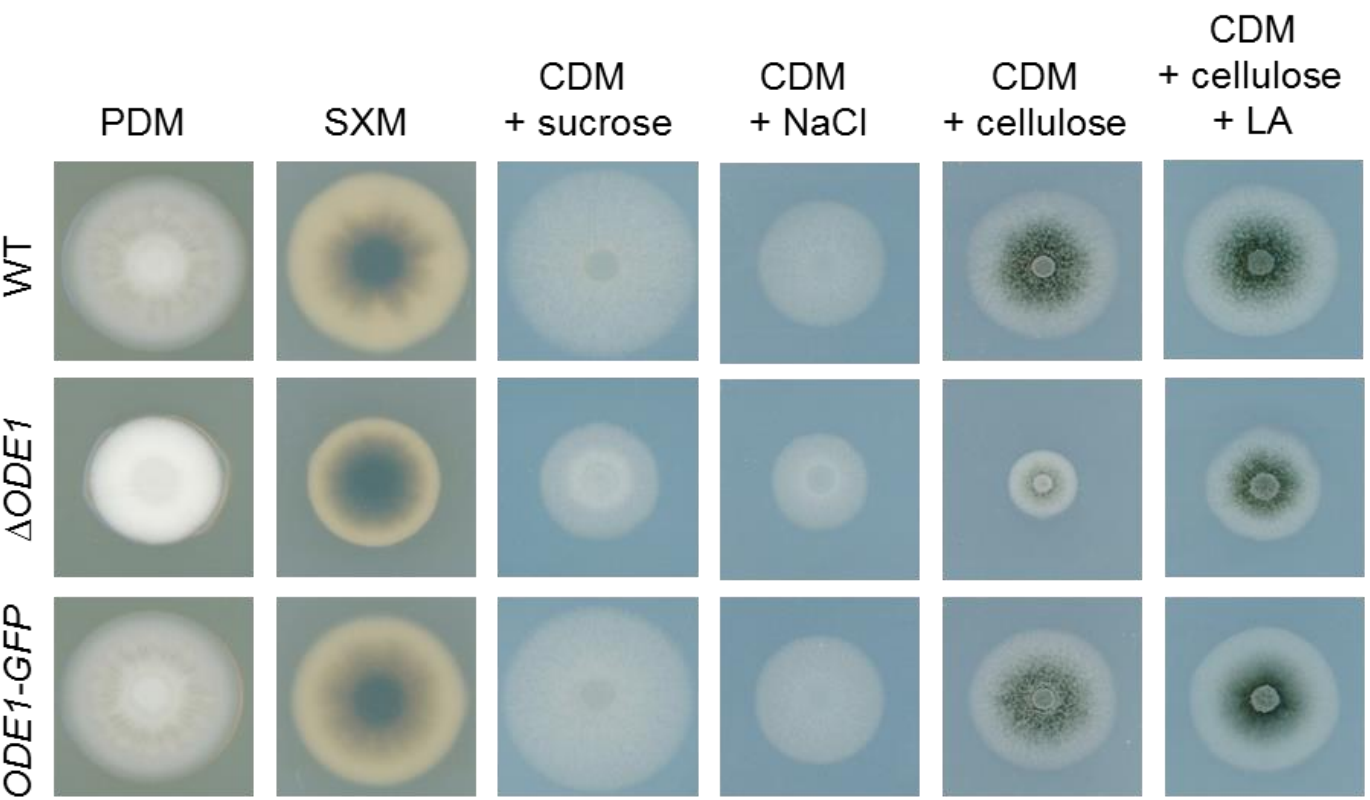

B
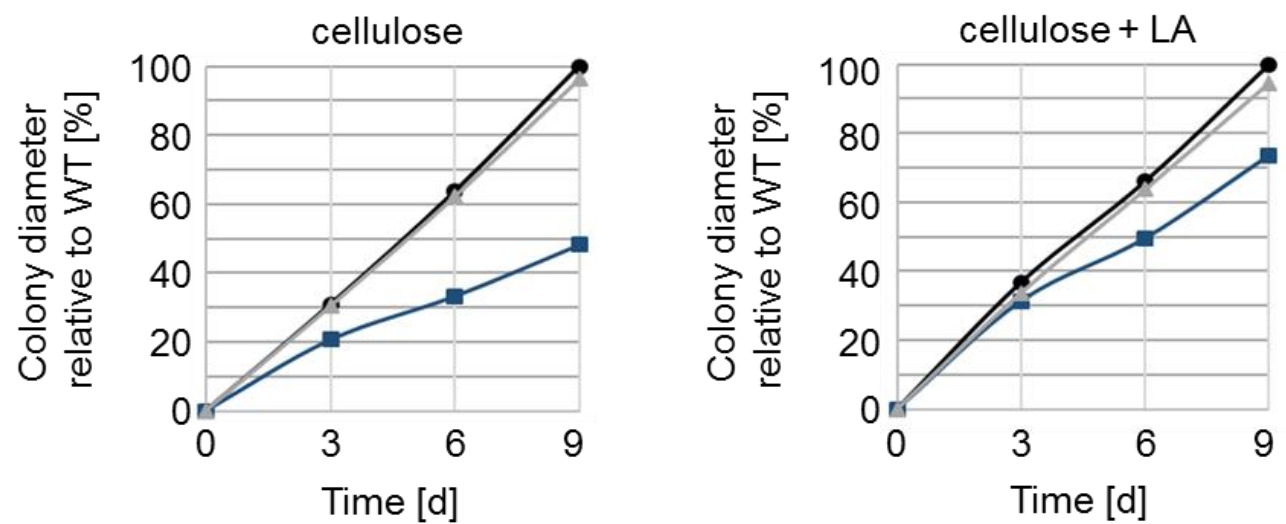

$\rightarrow$ WT

$\triangle O D E 1$

ODE1-GFP

Figure 26: V. dahliae ODE1 contributes to vegetative growth. (A) Ex planta phenotypes of the V. dahliae wild type JR2, $\triangle O D E 1$, and the ODE1-GFP complementation strain nine days after spot inoculation of 50000 spores on PDM, SXM, CDM supplemented with $\mathrm{NaCl}$ $(0.5 \mathrm{M}), \mathrm{CDM}$ with either sucrose or cellulose as carbon source, and CDM with cellulose supplemented with linoleic acid (LA; $0.125 \mathrm{mg} / \mathrm{ml}$ ) and incubation at $25^{\circ} \mathrm{C}$. (B) Growth quantification of the wild type JR2, $\triangle O D E 1$, and the ODE1-GFP complementation strain three, six, and nine days after spot inoculation of 50000 spores on CDM with cellulose as carbon source either without (left) or with supplementation of $0.125 \mathrm{mg} / \mathrm{ml} \mathrm{LA}$ (right). Growth was quantified by measurement of two perpendicular diameters per colony for three independent colonies of the wild type and two independent transformants per genotype (VGB331, VGB332, VGB358, VGB359) in two independent experiments with similar results. $\triangle O D E 1$ displayed $\sim 50 \%$ decreased growth in comparison to wild type and the ODE1-GFP strain about nine days after spot inoculation on CDM with cellulose. Supplementation of the medium with LA partially complements the growth defect of $\triangle O D E 1$ to $\sim 75 \%$ of the wild type level. 
Nine days after spot inoculation on complete medium $\triangle O D E 1$ showed a fluffier, whitish phenotype due to the production of more aerial mycelium compared to wild type and complementation strain ODE1-GFP. The supplementation of minimal medium with stress inducing agents resulted in decreased growth of all tested strains, but not especially in the $\triangle O D E 1$ strain as shown for sodium chloride in Figure $26 \mathrm{~A}$.

The ODE1 deletion strain displayed the most severe decrease in vegetative growth on minimal medium containing cellulose as carbon source with a $\sim 50 \%$ reduction of the colony diameter relative to wild type after nine days (Figure 26A, B). Colony diameters of ODE1-GFP were wild type-like (96\%). The observed growth defect of $\triangle O D E 1$ could be partially restored by supplementation of the medium with the oleate $\Delta 12$-fatty acid desaturase product linoleic acid, resulting in a colony diameter about $75 \%$. Again, ODE1-GFP displayed wild type-like colony diameters (95\%).

Alterations in melanization were observed for colonies formed by $\triangle O D E 1$. Colony cross sections of $\triangle O D E 1$ grown on minimal medium with cellulose displayed less melanized structures in the agar after nine days (Figure 27A).

Figure 27: V. dahliae ODE1 is involved in microsclerotia formation. Microsclerotia formed by the $V$. dahliae JR2 wild type, $\triangle O D E 1$, and the ODE1-GFP complementation strain after spot inoculation of 50000 spores. Two independent transformants per genotype were tested (VGB331, VGB332, VGB358, VGB359). Black scale bar $=1$ $\mathrm{mm}$, blue scale bar $=20 \mu \mathrm{m}$, red boxes and dashed lines = cross sections from colony centers. (A) Pictures from colonies (top), cross sections (middle) and microscopy of fungal material scraped from colony centers (bottom) after nine days on CDM with cellulose and incubation at $25^{\circ} \mathrm{C}$ are shown. (B) Colonies (top) and cross sections of colony centers (bottom) after 14 days on CDM with glucose (bottom view) and incubation at $25^{\circ} \mathrm{C}$ in the dark are shown.

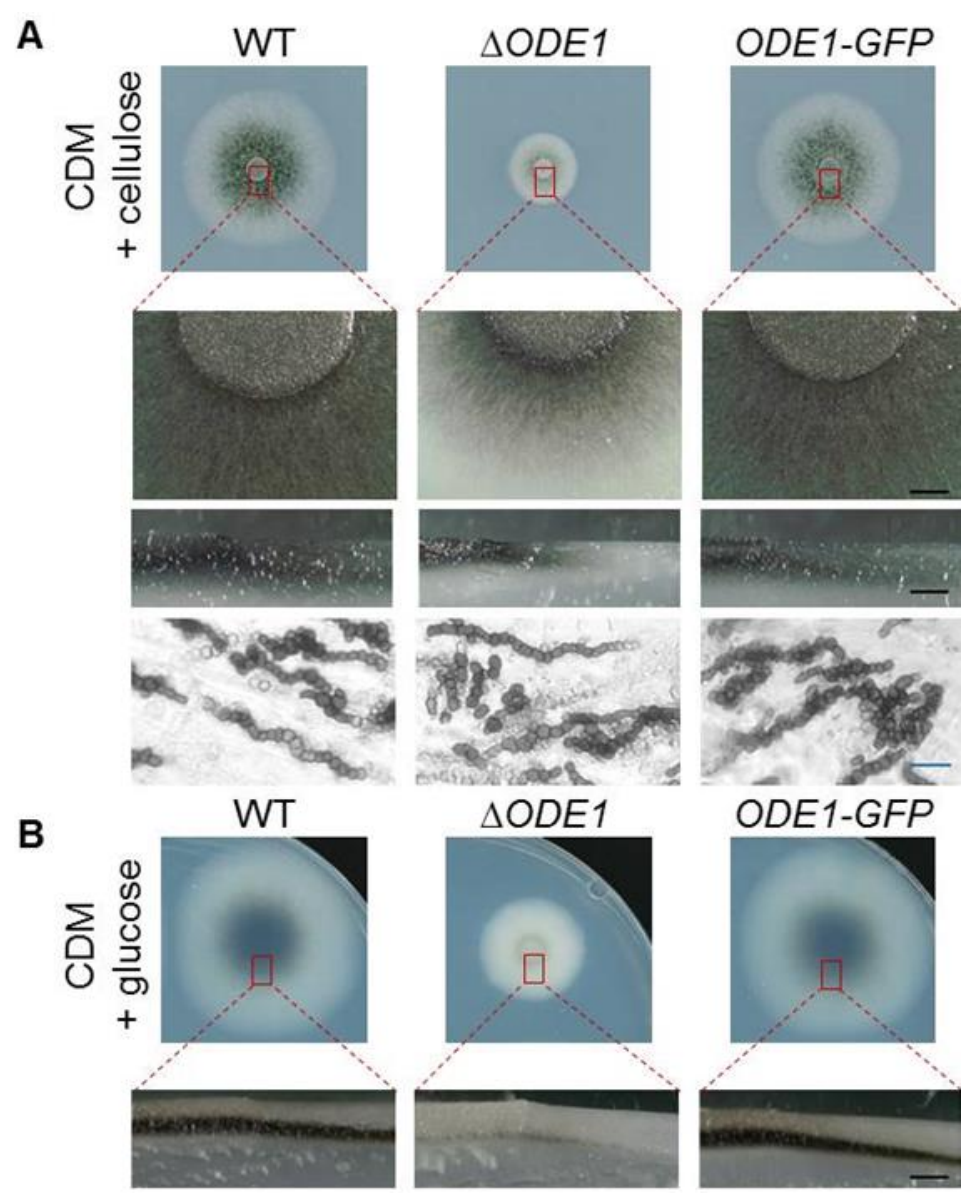


During microscopy of fungal material from colony centers of $\triangle O D E 1$, wild type-like microsclerotia could be observed. Changing the growth conditions to 14 days incubation in the dark on minimal medium with glucose, a decrease in microsclerotia production in $\triangle O D E 1$ became more apparent (Figure 27B).

Hence, $V$. dahliae Ode1 is essential for wild type-like development of the fungal colony and shows positive impacts on microsclerotia formation. However, Ode1 is dispensable for stress responses under tested conditions. The C-terminal GFP fusion to Ode1 allowed complementation phenotypes close to wild type levels. The observed growth defect of $\triangle O D E 1$ was partially restored in presence of extracellular linoleic acid.

\subsubsection{V. dahliae ODE1 is dispensable for induction of disease symptoms in tomato plants}

The ODE1 deletion strain was less impaired in growth in presence of linoleic acid ex planta. Also plants synthesize linoleic acid as an important precursor of defense-related compounds like plant oxylipins and cutin monomers and, therefore, might substitute the defect in fungal linoleic acid biosynthesis (Soliday \& Kolattukudy, 1977; Brodhun \& Feussner, 2011). During tomato plant infection experiments the impact of the ODE1 deletion on virulence of $V$. dahliae was investigated.

Tomato plants inoculated with spores obtained from the $\triangle O D E 1$ strain displayed overall similar stunting and hypocotyl discolorations to wild type-infected plants with only slightly reduced numbers of plants with disease symptoms after 21 days. About $70 \%$ of the tomato plants inoculated with $\triangle O D E 1$ spores displayed disease symptoms in comparison to $87 \%$ of the wild type treated plants (Figure 28 ).

In summary, the ODE1 deletion strain displayed generally impaired vegetative growth, correlated with decreased microsclerotia production and allows almost wild type-like induction of disease symptoms. Hardly any decrease in induced disease symptoms was observed in plants inoculated with spores obtained from the ODE1 deletion strain suggesting a minor or no role of the single gene ODE1 in virulence of $V$. dahliae. This virulent in planta phenotype of a strain with a severe growth defect might result from substitution of the fungal ODE1 product by linoleic acid provided in plant cells. 


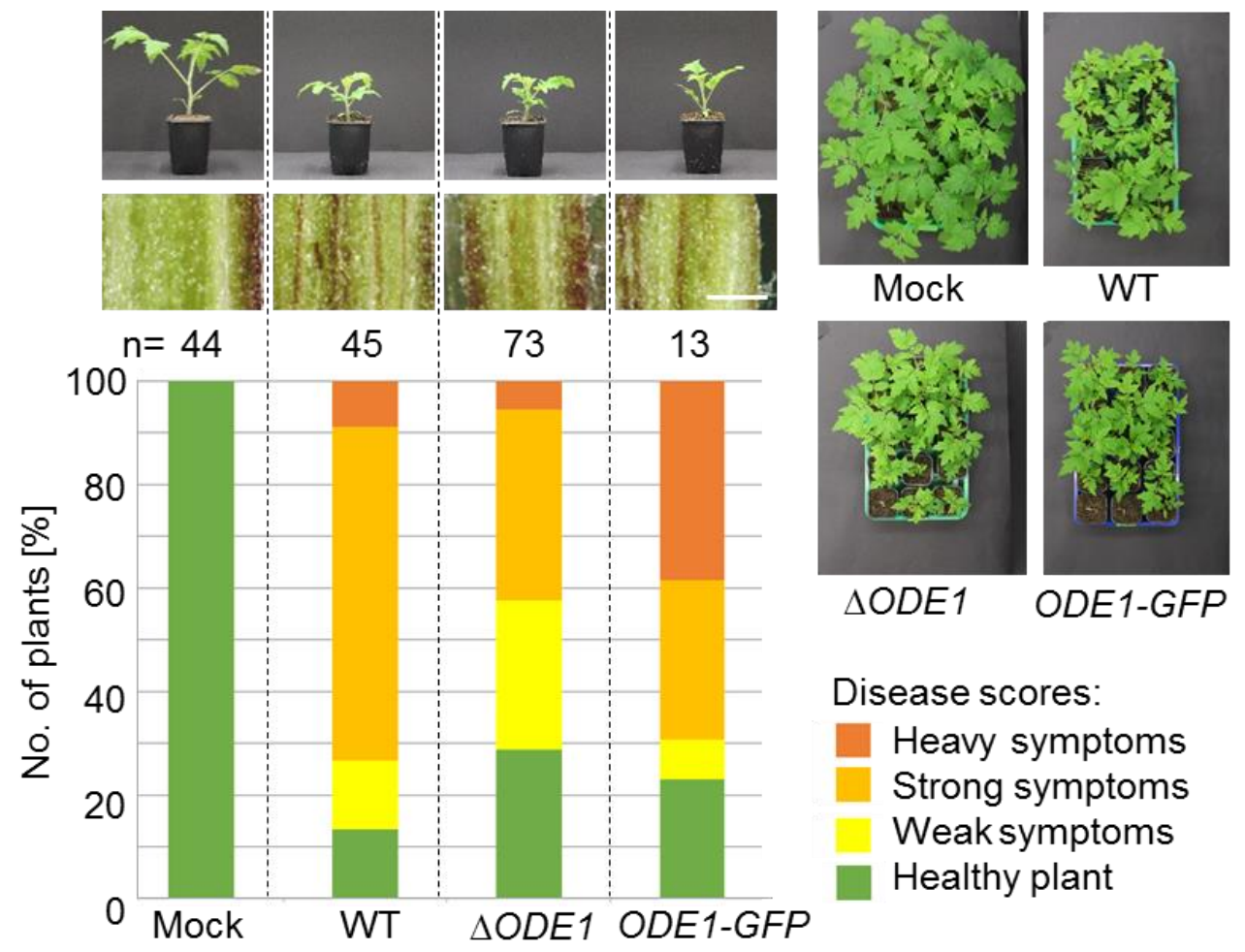

Figure 28: $V$. dahliae $O D E 1$ is not required for induction of severe disease symptoms in S. Iycopersicum. Ten-day-old tomato plants were inoculated by root dipping into water control (mock) or spores obtained from wild type JR2 (WT), $\triangle O D E 1$ (VGB331, VGB332), or ODE1-GFP complementation (VGB359) strains. Representative plants and overview of 15 plants per genotype from one experiment are shown. Disease symptoms were assessed after 21 days and transferred into disease scores per plant visualized in a stack diagram. Discoloration of hypocotyl dissections is shown. Data are shown from three independent experiments for wild type and $\triangle O D E 1$ (VGB331), two independent experiments for a second $\triangle O D E 1$ transformant (VGB332), and a single experiment for ODE1-GFP. Scale bar $=1 \mathrm{~mm}$, $\mathrm{n}=$ total number of evaluated plants per genotype. 


\section{Discussion}

The outcome of a host-fungus interaction can be fungal colonization due to plant susceptibility or fungal death combined with plant resistance. Between these two extremes several intermediate outcomes of disease severity can be observed. Several factors affect this relationship between a pathogen and a host, including environmental factors, nutritional conditions, age, and genome variations of both, plant and fungus. Fungal signaling genes were in the focus of this study. The aim was a better understanding of the complex interplay of fungal signaling cascades favoring beneficial or detrimental outcomes in interactions with host plants.

It was shown, that pathogenic $V$. longisporum isolates possess genomic insertions which contribute to attenuation of disease symptoms induced in the interplay with rapeseed plants. These regions can be either species- or host-specific.

Virulence of $V$. dahliae is mediated by MAPK signaling pathways, but the role of scaffold proteins in insulation of these pathways was yet unstudied in plant pathogens. Ham5 is a MAPK scaffold in different ascomycetes. Here it was shown, that MAPK signaling pathways in $V$. dahliae mediate differentiation, stress response, and virulence independent from this scaffold protein.

Plant invasion and suppression of the plant defense responses rely on the secretion of certain tools which can be regulated by the UPR pathway. Therefore, the $V$. dahliae bZIP transcription factor Hac1 and its role in virulence was characterized in this study. The UPR regulator has conserved as well as species-specific impacts on differentiation of V. dahliae and is an important factor for virulence.

Virulence can be connected to fungal differentiation. Important regulators of fungal differentiation are lipid metabolites which can act as signaling molecules. Characterization of the fungal oleate $\Delta 12$-fatty acid desaturase Ode1, catalyzing the synthesis of linoleic acid, revealed an important contribution to fungal growth with only a minor impact on the induction of disease symptoms.

Figure 29 represents an overview about the findings of this thesis, which will be discussed in detail in the following. 


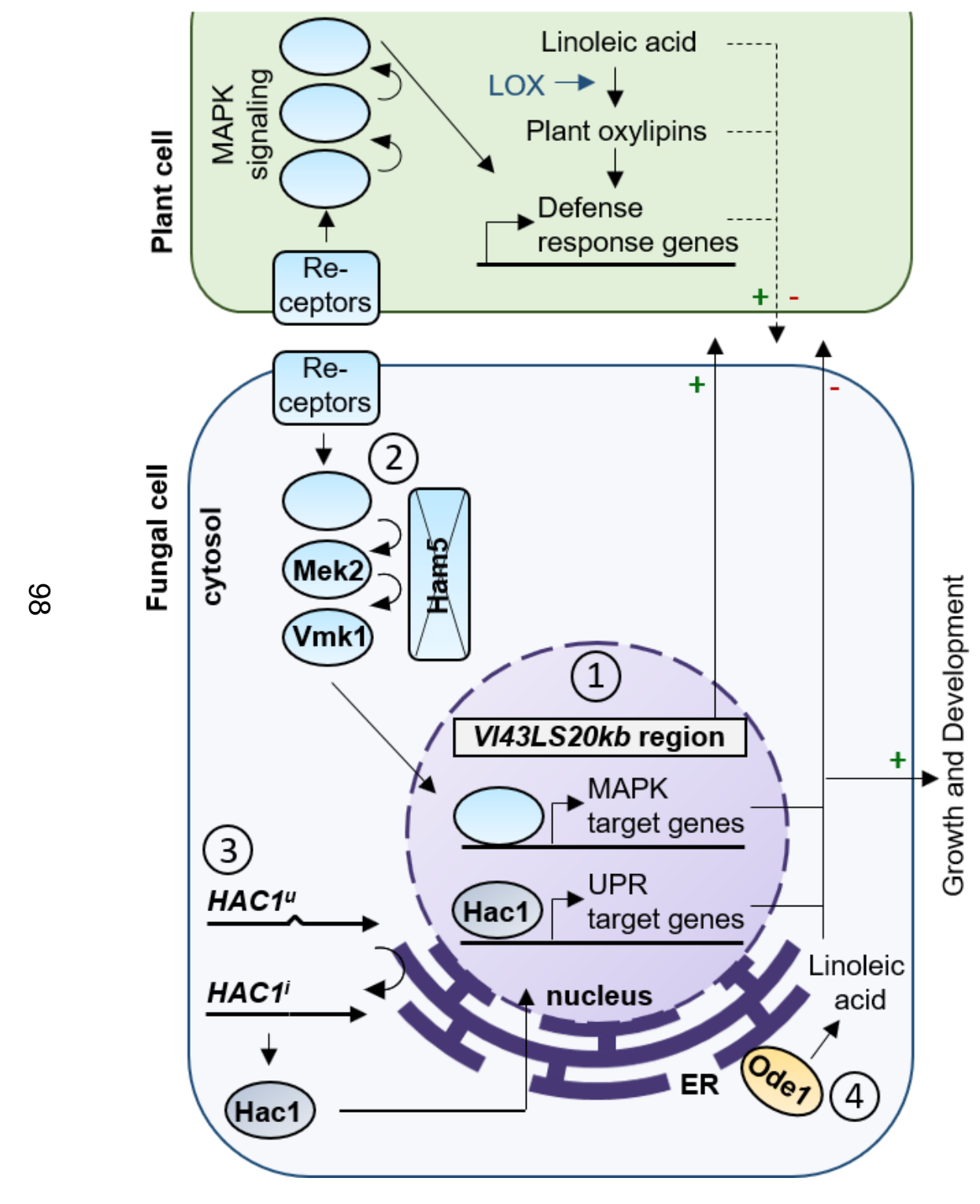

Figure 29: Interactions between haploid V. dahliae and amphidiploid $V$. longisporum isolates with their plant hosts studied in this thesis. (1) The pathogenic $V$. longisporum isolate VI43 possesses the genomic region VI43LS20kb, which is absent in the genome of the nonpathogenic isolate VI32. This region contributes to attenuation of disease symptoms induced in the interplay with rapeseed plants (indicated by arrow with green plus from fungal cell and arrow with dashed line and red minus from plant cell). (2) The $V$. dahliae Vmk1 MAPK pathway components Mek2 (MAP2K) and Vmk1 (MAPK) positively control vegetative growth and microsclerotia formation (indicated by arrow with green plus to "Growth and Development") and are required for induction of disease symptoms in plants (indicated by arrow with red minus from fungal cell) independent from the scaffold homolog Ham5 in V. dahliae. The MAPK pathway is required for initial penetration and for the susceptibility of the plant (indicated by arrow with dashed lines and green plus from plant cell). (3) Upon interaction with the host, the fungal cell has to cope with an increased demand for secreted proteins, which leads to ER stress. The uninduced mRNA of the UPR regulator Hac1 $\left(H A C 1^{4}\right)$ is unconventionally spliced (indicated by arrow close to ER membrane) and the $H A C 1^{i}$ mRNA is translated into the bZIP transcription factor Hac1, which regulates UPR target genes. $V$. dahliae HAC1 is involved in the ER stress response and vegetative growth under non-stress conditions, has a strong impact on conidiation, and is essential for the formation of microsclerotia (indicated by arrow with green plus to "Growth and Development"). HAC1 is required for the virulence of $V$. dahliae (indicated by arrow with red minus from fungal cell) and potentially regulates expression or secretion of effector proteins, which enables the fungus to circumvent plant defense responses (indicated by arrow with dashed line and green plus from plant cell). (4) The oleate $\Delta 12$-fatty acid desaturase Ode1, which catalyzes the synthesis of linoleic acid, supports fungal growth and microsclerotia formation (indicated by arrow with green plus to "Growth and Development"). Ode1 deficiency has only minor effect on development of disease symptoms in planta, which might be due to the availability of plant linoleic acid that potentially supports fungal growth and differentiation (indicated by arrow with dashed line and green plus from plant cell). 


\subsection{The pathogenic V.longisporum isolate VI43 possesses a genomic insertion contributing to disease symptom attenuation in rapeseed plants}

\subsubsection{V. longisporum isolates colonize plants with different pathotypes}

$V$. longisporum is an amphidiploid interspecies hybrid, which evolved from at least three separate hybridization events (Inderbitzin et al., 2011b; Figure 2). All known $V$. longisporum lineages arose by hybridization of the haploid species $A 1$ with one of three V. dahliae or V. dahliae related strains D1, D2, or D3 (Inderbitzin et al., 2011b). Isolates from the A1/D3 lineage were described overall as the least pathogenic in comparison to the lineages $A 1 / D 1$ and $A 1 / D 2$. However, not all isolates of the $A 1 / D 3$ lineage colonize rapeseed without induction of disease symptoms (Novakazi et al., 2015). In a pathogenicity test involving seven isolates from the lineage $A 1 / D 1$, four $A 1 / D 2$, and four $A 1 / D 3$, a single $A 1 / D 3$ isolate was the most virulent on rapeseed (Novakazi et al., 2015). In this study, the tested A1/D3 strain VI32 colonized rapeseed plants without induction of any disease symptoms, whereas the A1/D1 isolate VI43 induced severe disease symptoms under laboratory conditions (Figure 7). Both isolates were able to enter and propagate within the plants with different outcomes for plant health. Less fungal outgrowth could be observed from stem sections of the asymptomatic strain $\mathrm{VI32}$, hinting to reduced propagation and reduced fungal biomass within the plant. This might be either due to plant mechanisms reducing fungal growth or a less aggressive colonization behavior of VI32. Previous experiments comparing VI43 and VI32 root colonization did not show differences of the fungal behavior prior to entering the plant on the root surface. Accordingly, propagation of VI32 within the plant following initial penetration of the roots must be affected. The comparison of the ex planta phenotypes of $V$. longisporum isolates from different lineages revealed a difference in the growth behavior of strains from the A1/D1 towards the A1/D3 lineage on malt extract agar. A1/D3 strains VI32 and VI19 formed bundles of single hyphae, which were covering the colonies. These bundles were rare in the A1/D1 strains VI43 and VI145c. Since Verticillia colonize the vascular system of the host plant and occurrence of disease symptoms is correlated with clogging of vessels hindering the transpiration stream (Yadeta \& Thomma, 2013), the formation of arranged hyphal bundles might have a less disturbing influence on the flow of the xylem fluid in VI32 colonized plants. An impact of directed hyphal growth on the pathotype of plant colonizing fungi was observed in the mutualistic grass colonizing Epichloë festucae. Here, hyphae show parallel growth aligned with elongating leaf cells (Christensen et al., 2008). A mutant deficient in the reactive oxygen producing membrane-bound Nox complex (nicotinamide adenine 
dinucleotide phosphate oxidase complex), displayed pathogenic development correlated with hyperbranched hyphal growth which was no longer tightly connected to the leave cells (Brown et al., 2016; Tanaka et al., 2006, 2008; Takemoto et al., 2006, 2011). The formation of hyphal bundles in isolates of the $V$. longisporum A1/D3 lineage was observed only on malt extract agar, which is not very similar to the conditions of low nutrient availability given within the vascular system of rapeseed plants. If hyphal bundles are formed within the plant and influence the transpiration stream remains to be elucidated.

\subsubsection{The $V$. longisporum VI43LS2Okb region mediates virulence attenuation towards rapeseed plants}

Investigation of genomic differences between $V$. longisporum isolates with different pathotypes revealed a high conservation on gene level, but differences in the chromosome numbers and the karyotypes. The additional chromosome observed in the asymptomatic isolate $\mathrm{VI} 32$ was not an accessory chromosome comprising genes that are species-specific, but a result of rearranged genomic regions. Occurrence of frequent synteny breakpoints caused by genomic rearrangements was observed in all Verticillia (Shi-kunne \& Faino, 2017). Chromosomal reshuffling in eukaryotes is correlated with alterations in phenotypes and is assumed to confer varying fitness in different niches by gene gains or losses (Coghlan et al., 2005; Tang \& Amon, 2013; Dong et al., 2015; Plissonneau et al., 2018). In the haploid species $V$. dahliae a correlation between genomic rearrangements and the evolution of Lineage Specific regions was observed (Klosterman et al., 2011; de Jonge et al., 2013; Chen et al., 2018; Faino et al., 2016; Gibriel et al., 2019). The V.dahliae VdLs.17 LS regions 1-4 were proposed to be involved in adaptation of $V$. dahliae to different host niches and in its host range expansion in comparison to $V$. alfalfae (Klosterman et al., 2011). A genomic LS region originating from the parental lineage related to $V$. dahliae is present in the pathogenic isolates VI43 and VI145c and absent in the nonpathogenic V. longisporum isolate VI32 (Figure 8). This finding may lead to the assumption that this region contributes to fungal virulence. However, characterization of the VI43LS20kb region unraveled a negative impact on the severity of disease symptoms induced in V. longisporum VI43 colonized rapeseed plants. The significant effect of this region was shown in three different strains deficient in the entire VI43LS20kb region or in either the first or the second part of this region. All of the VI43 LS deletion strains induced more severe disease symptoms in rapeseed plants than the wild type (Figure 9, Figure 30).

The different $L S$ deletions did not cause obvious alterations in their ex planta phenotypes in comparison to the wild type VI43 (Figure S2). Increased fungal growth or propagation 
with detrimental impact on the host plant cannot be considered as an explanation for the hypervirulent in planta phenotype. Instead, negative impacts on pathogenicity might be explained with the hemibiotrophic life style of pathogenic Verticillium strains, performing a switch from an initial biotrophic stage to a later necrotrophic stage (Fradin \& Thomma, 2006). These stages require differentiated strategies in the crosstalk with the host plant. Fungal tools used to modulate the interaction with the plant can be divided into two groups: those secreted during biotrophic interactions and those, which induce the necrotrophic phase (O'Connell et al., 2012; Lo Presti et al., 2015; Chini et al., 2018; Gibriel et al., 2019). During the biotrophic interaction, the host defense responses are, for example, suppressed by effectors and secondary metabolites. Induction of the necrotrophic stage is correlated with the secretion of lytic enzymes, oxylipins, or fungal effectors which are activating the plant defense responses, induce cell death, and facilitate fungal nutrient uptake (O'Connell et al., 2012; Lo Presti et al., 2015; Chini et al., 2018; Gibriel et al., 2019). Mis-regulation of the tools required for induction of the necrotrophic phase can lead either to a lack of this phase, a premature induction, or rather increased secretion of the respective tools, which might result in hypervirulence. In our case, this means that expression or secretion of tools required to induce the necrotrophic phase might be decreased by certain factors encoded in the VI43LS20kb region in the wild type VI43. These pathogenicity attenuating factors would make the pathogenic isolate more tolerable for rapeseed plants and would not be required in the asymptomatic colonizer VI32, due to the absence of the respective targeted tools for induction of the necrotrophic phase.

The VI43LS2Okb region, identified in the pathogenic rapeseed colonizer VI43, encodes six hypothetical small proteins and one larger protein with cytosolic, nuclear, or transmembrane localization, but without secretion signals (Figure 8B). For Lsg1, Lsg2, Lsg4, and Lsg7 no domains were predicted. The absence of a predicted signal peptide makes the secretion of these proteins via the ER/Golgi secretory pathway not very likely. Alternatively, unconventional secretion mechanisms independent from secretion signal sequences exist in fungi (Miura \& Ueda, 2018). Unconventional secretion is described, for example, for fungal enzymes with effector properties, such as an isochorismatase in Phytophthora sojae and $V$.dahliae. The isochorismatase manipulates defense responses by suppression of salicylic acid accumulation in the host plant (Liu et al., 2014). One should consider that examples for mutants deficient in effectors with hypervirulent phenotypes in fungi are rare and, therefore, the presence of effector encoding genes in the VI43LS20kb region might be less likely. In U. maydis 12 effector gene clusters were identified, encoding genes upregulated simultaneously in infected plant tissue (Kämper et al., 2006). Absence of these clusters resulted in decreased 
virulence in four cases and in unaffected virulence in seven cases. However, hypervirulence was observed following disruption of the Um01234-41 effector gene cluster (Kämper et al., 2006). The mechanism explaining this phenotype has not been elucidated yet.

To date, research on LS regions correlated with synteny breakpoints in Verticillia was mainly restricted to identified effectors promoting fungal virulence (de Jonge et al., 2013; Faino et al., 2016; Kombrink et al., 2017; Li, 2019, Dissertation, University Wageningen). Effector gene enrichment in LS regions relative to the core genome was found by comparison of different $V$. dahliae isolates (de Jonge et al., 2013; Gibriel et al., 2019). In contrast, LS and core genomes from different species of the genus displayed the same relative numbers of genes encoding proteins with secretion signals (Klosterman et al., 2011). The number of effector genes relative to the total number of genes encoded in the LS regions of $V$. dahliae strains was rather small (de Jonge et al., 2013; Gibriel et al., 2019). Besides effector protein encoding genes other genes in LS regions might provide potential for niche adaptation.

Lsg3 and Lsg6 encoded by the VI43LS20kb region contain predicted transcription factor domains. Lsg3 is encoded in the LSI subregion, whereas Lsg6 is encoded in the LSII subregion. Downregulation of virulence-related genes has been observed during transcriptome analysis of related fungal species with different pathotypes. Recently, comparison of the transcriptomes of a $V$. dahliae strain, which causes less severe disease symptoms in cotton in comparison to a more virulent relative, revealed downregulation of pathogenicity-related genes as ,for example, genes involved in hydrophobin and melanin production (Jin et al., 2019). Genome comparison of the same $V$. dahliae strains displayed an enrichment of genes bioinformatically predicted as transcription factors. These transcription factors could downregulate pathogenicityrelated genes (Jin et al., 2019). Another example from different Colletotrichum species corroborates that differentiated transcriptional regulation during host colonization can decide about the life style of plant colonizing fungi resulting in different pathotypes, which are not necessarily associated with extensive remodeling of the gene repertoire (Hacquard et al., 2016). A limited activation of pathogenicity-related genes in planta was observed for the endophytic species $C$.tofieldiae in comparison to the pathogenic relative $C$. incanum (Hacquard et al., 2016). Transcription factors, which are negatively regulating virulence, have been characterized in A. brassicicola and F. oxysporum (Caracuel et al., 2003; Cho et al., 2012; Brown et al., 2016). In F. oxysporum the pH signaling transcription factor $\mathrm{PacC}$ negatively regulates genes expressed under low $\mathrm{pH}$ conditions and mutants deficient in this repressor display increased virulence in tomato plants (Caracuel et al., 2003). Further, hydrolytic enzymes are transcriptionally repressed 
by the A. brassicicola transcription factor Amr1 (Alternaria melanin regulation 1) known for its role in melanin biosynthesis in other plant pathogenic fungi (Cho et al., 2012). The role of this transcription factor in virulence is unique for A. brassicicola and was not observed for homologs as V. dahliae VdCmr1 (V. dahliae Colletotrichum homologous melanin regulation 1; Cho et al., 2012; Brown et al., 2016; Wang et al., 2018).

For Lsg5 encoded in the VI43LS20kb region, a Zinc finger RING-type domain was predicted (Figure 8B). Zinc finger RING-type domains mediate protein-protein interactions or ubiquitin transfer either to substrates or the Zinc finger RING-type domain protein itself (Joazeiro \& Weissman, 2000). The protein possesses an additional transmembrane domain, with a short non-cytosolic and a longer cytosolic region containing the RING domain. The yeast scaffold protein Ste5 of the pheromone response pathway contains a RING-domain required for its interaction with Ste4, a subunit of the G-protein coupled to the transmembrane receptors of the mating pathway (Inouye et al., 1997). Orthologous Ham5 scaffold proteins in ascomycete filamentous fungi do not possess a RING-domain (Figure 11). The regulation of signaling cascades assembled at the membrane following perception of external signals might involve RINGdomain transmembrane proteins, such as the protein Lsg5 encoded in the VI43LS20kb region.

Degradation of proteins located in plasma membranes, like receptors, transporters, and channels, by endocytosis is generally triggered by ubiquitination (Piper \& Luzio, 2007; Nakamura, 2011). This could be also true for soluble proteins interacting with complexes associated to the membrane. In mammals, Membrane-Associated RING-CH (MARCH) proteins are a well described family of RING-domain proteins, which are located to cell membranes and are important for ubiquitination of cell-surface immune molecules (Goto et al., 2003; Nakamura, 2011; Samji et al., 2014; Zhang et al., 2019). The absence of ubiquitin ligases located to the fungal plasma membrane might result in increased occurrence of certain membrane or membrane-complex-associated proteins. An adaptation of the fungal membrane proteome following interaction with the host plant's cell wall was described in the plant pathogen B. cinerea (Liñeiro et al., 2016). This adaptation might be disturbed in the absence of membrane-bound ubiquitin ligases responsible for degradation of membrane proteins. An increased occurrence of membrane-complex-associated proteins, such as co-receptors or activators of signaling cascades, in absence of a membrane-bound ubiquitin ligase could result in a permanent activation of cascades. This permanent cascade activation might promote virulence, for example by increased secretion of virulence factors.

Defects in signaling cascades important for sensing of the environment, signal transduction, and induction of infection-related morphogenesis result in hypervirulence in 
fungi (Brown et al., 2016). In the basidiomycete $U$. maydis the phosphatase Rok1 (regulator of Kpp2) controls dephosphorylation of the partially redundant Fus3/Kss1-like MAP kinases Kpp2 and Kpp6 (kinase PCR-product 2/ 6), which regulate efficient appressorium formation and plant invasion in a negative feedback loop (Di Stasio et al., 2009). U. maydis rok1-deficient mutants displayed a hypervirulent phenotype on maize plants correlated with more efficient appressorium formation. As previously mentioned, Nox complex-deficient mutants of $E$. festucae induce disease symptoms in planta correlated with hyperbranched hyphal growth with increased fungal biomass (Tanaka et al., 2006; Takemoto et al., 2006; Tanaka et al., 2008; Takemoto et al., 2011; Brown et al., 2016). In the same fungus, the Hog1-like MAPK signaling cascade is involved in downregulation of hydrolytic enzyme secretion and mutants deficient in the MAPK display virulence on their natural symbiotic hosts (Eaton et al., 2010; Brown et al., 2016). In summary, Lsg3 and Lsg6 have the potential to act as transcription factors with roles in downregulation of mechanisms required for induction of disease symptoms. Lsg5 could regulate signal transduction from the membrane via protein-protein interactions or ubiquitination, targeting components to proteasomal degradation. Further, proteins encoded in the VI43LS20kb region might act as effector proteins, which are unconventionally secreted. The mechanism leading to hypervirulence following deletion of the VI43LS20kb region in VI43 still remains to be elucidated. This is the first study unraveling an LS region in Verticillia which attenuates virulence in a pathogenic isolate. The absence of this region in the asymptomatic isolate VI32 shows that not only pathogenicity factors or the loss of genomic regions, but also regions involved in attenuation of pathogenicity, contribute to the differences in the genomes of isolates from different pathotypes.

\subsubsection{Host specificity of virulence and putative attenuating factors in Verticillia}

In this study, an LS region with functions in attenuation of disease symptoms in the interaction of $V$. longisporum with rapeseed plants was identified. The pathogenesisreducing mechanism of this region depends either on the fungal species or the host.

The genome of the haploid $V$. dahliae isolate JR2 contains a VI43LS20kb homologous region and VI43LS20kb homologous subregions could be identified rearranged in the genome of the $V$. dahliae strain VdLs.17. In contrast, the VI43LS20kb region is absent from the genome of $V$. alfalfae VaMs.102, which is more closely related to the $V$. longisporum parental lineage $A 1$, and from the $V$. longisporum isolate $V I 32$ (A1/D3). These findings corroborate the origin of the VI43LS20kb region in the genome of the $V$. longisporum lineage A1/D1 parental species D1. 
The VI43LS20kb homologous region encoded in the genome of $V$. dahliae JR2 displays an indifferent role in pathogenicity towards tomato plants (Figure 10, Figure 30). The $V$. dahliae JR2 wild type does not induce disease symptoms in rapeseed plants. Two $V$. dahliae isolates were able to colonize the roots of rapeseed plants, but the isolates remained in the lower parts of the plants without induction of disease symptoms (Zhou et al., 2006). Whether the homologous VI43LS20kb region in V. dahliae affects the colonization behavior and pathogenicity of this species against rapeseed or other host plants is currently unknown.

The genome of $V$. dahliae JR2 harbors an additional copy of the gene LSG1 encoded in the VI43LS20kb homologous region, which was not deleted in V. dahliae JR2 $\Delta L S$. Since all constructed deletion strains in $V$. longisporum VI43, $\Delta L S I, \Delta L S I$, and $\Delta L S$, resulted in induction of more severe disease symptoms in rapeseed plants and the phenotype was even stronger in $\Delta L S /$ than in $\Delta L S I$ (Figure 9), the presence of an additional copy of LSG1 located in the LSI subregion does not represent a sufficient explanation for the unaltered pathogenicity of $V$. dahliae JR2 in absence of the homologous VI43LS20kb region.

Even if $V$. dahliae species have a broad host range, the single isolates of the species differ in their host ranges and in virulence towards different host plants (Resende et al., 1994; Zeise \& von Tiedemann, 2002; Pegg \& Brady, 2002; Gibriel et al., 2019). For isolates of the same $V$.longisporum lineage, differences in their pathogenicity on different host plants was observed as well (Novakazi et al., 2015). Therefore, one can suggest that strains of both species generally display a high degree of specialization and adaptation to a certain host. This specialization might not always be the result of the acquirement of novel genes, but also the differential regulation of existing mechanisms in response to certain environmental factors.

In other plant colonizing fungi, gene losses due to chromosomal rearrangements with significant roles in niche adaptation were described (Sharma et al., 2014; Hartmann et al., 2017; Plissonneau et al., 2018), which might be a possible mechanism in Verticillia as well. Further, asymptomatic or less virulent isolates might contain several pathogenicity-related genes in their genomes, which are inactive due to certain regulatory mechanisms.

Several studies investigating LS regions from subgroups of $V$. dahliae isolates revealed effector genes required for colonization of particular hosts as, for example, the effector NLP-2 (necrosis and ethylene-inducing peptide 1-like protein 2) which is involved in virulence of the strain JR2 on tomato and $A$. thaliana, but dispensable for tobacco infection (Santhanam et al, 2013). The $V$. dahliae effector Tom1 is essential for tomato 
infection only and the effector Sun1 contributes to virulence specifically in sunflower colonization ( $\mathrm{Li}, 2019$, Dissertation, University Wageningen).

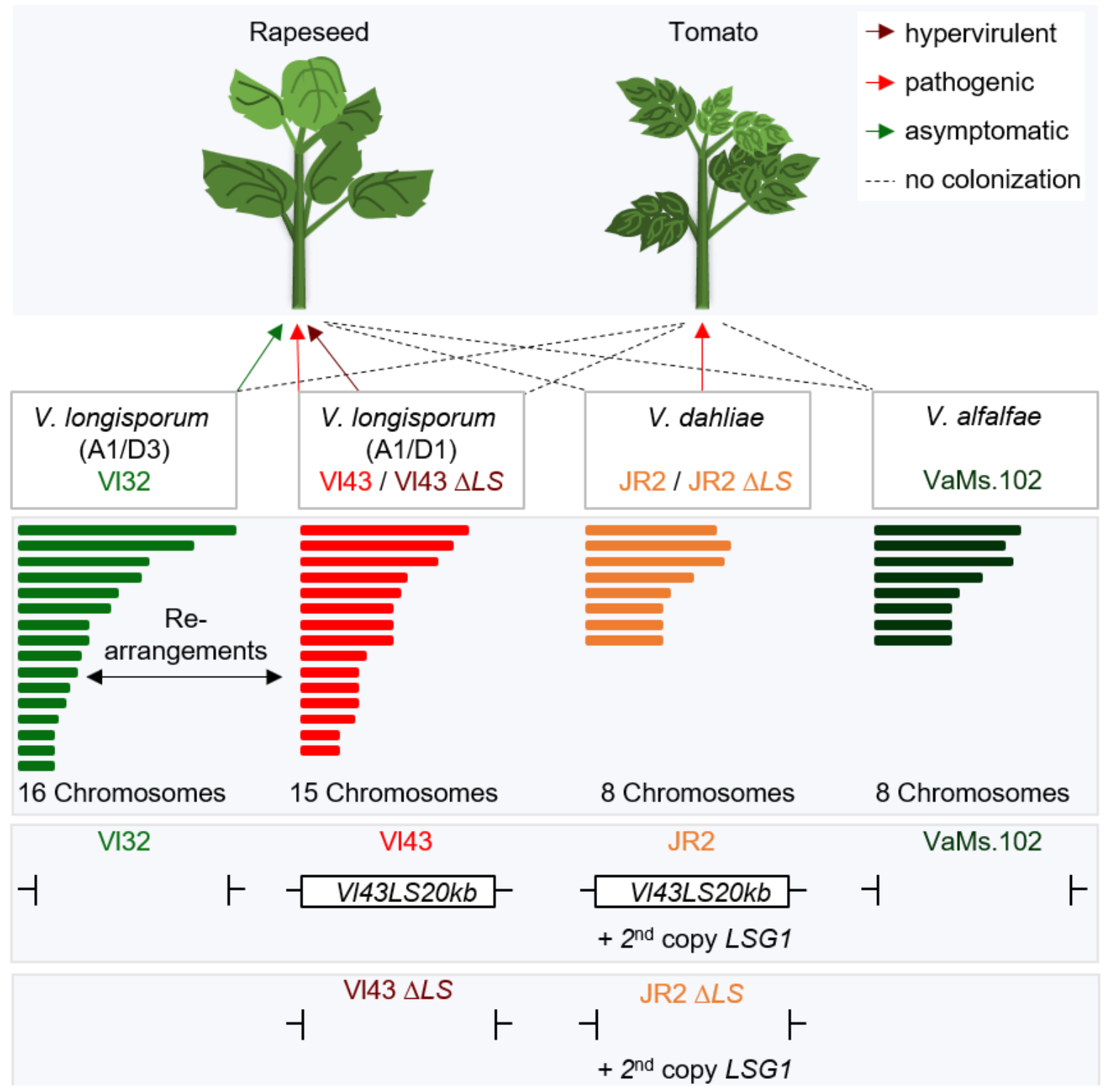

Figure 30: The VI43LS2Okb region attenuates pathogenicity of $V$. longisporum VI43 in rapeseed and the homologous region is indifferent for virulence of $V$. dahliae JR2 in tomato plants. The genomes of $V$. longisporum lineages evolved by hybridization from two haploid parental species, which are $V$. dahliae strains (D2, D3) or close relatives of $V$. alfalfae (A1) and $V$. dahliae (D1). The hybrid genomes differ in their karyotypes due to chromosomal rearrangements and display different chromosome numbers (15 for VI43; 16 for VI32). The species V. dahliae JR2 and V. longisporum VI43 (A1/D1) harbor the VI43LS2Okb region in their genomes, which is absent in V. longisporum VI32 (A1/D3) and V. alfalfae VaMs.102. V. dahliae JR2 exhibits an additional copy of the gene LSG1. Deletion of the VI43LS20kb homologous region in $V$. dahliae JR2 region results in an unaltered virulent phenotype on tomato plants (indicated by the light red arrow for both, the wild type and the $L S$ deletion strain), whereas deletion of the VI43LS20kb region in V. Iongisporum VI43 results in hypervirulence (indicated in dark red), suggesting a species- or host-specific role in attenuation of disease symptoms. $V$. longisporum isolates are unable to colonize tomato as a host (indicated by dashed lines), whereas V. dahliae remains in the roots without induction of disease symptoms and is not able to colonize the entire plant (indicated by dashed lines; Zhou et al., 2006). V. alfalfae colonizes alfalfa and hops and is not known as a colonizer of tomato or rapeseed plants (indicated by dashed lines; Bhat \& Subbarao, 1999). 
Further, seven LS genes acquired by horizontal gene transfer from F. oxysporum, contributed to the virulence of $V$. dahliae Vd991 in cotton, but were dispensable for virulence on tomato and lettuce (Chen et al., 2018). Integration of these genes into $V$. dahliae strains JR2 or VdLs.17 resulted in increased virulence on cotton, whereas there was no effect on tomato or lettuce hosts (Chen et al., 2018). The underlying mechanisms regulating the observed host-specific induction of disease symptoms is still unknown for all of these examples and remains to be elucidated. One could assume that fungal virulence factors, which are inducing disease symptoms in a certain host, target host-specific mechanisms for regulation of defense responses. The absence of the corresponding target in a different host would make the fungal tool dispensable. Further, recognition of host-specific external signals sent by the plant might be essential for induction of a fungal mechanism that is regulating its virulence.

In summary, the VI43LS20kb region makes V. longisporum VI43 more tolerable for rapeseed plants. Presence of the homologous region in $V$. dahliae JR2 is indifferent for induction of disease symptoms in tomato plants. Different impacts of the genomic region on the host-fungus interaction might be caused either by differential gene regulation in the different organisms, by host dependent gene regulation, or by host-specific targets of virulence factors regulated by the VI43LS20kb region.

\subsection{MAPK signaling pathways in $V$. dahliae mediate differentiation, stress response and virulence independent from the scaffold protein Ham5}

\subsubsection{The Ham5 scaffold function for the Fus3/Kss1-like MAPK cascade is not conserved among filamentous ascomycetes}

The perception of environmental signals and subsequent signal transduction are essential to induce adaptation processes in filamentous fungi. For example, sensing of a suitable host has to be forwarded to activate fungal colonization-related mechanisms. The Vmk1 MAPK signaling pathway in $V$. dahliae has a conserved role in fungal pathogenicity (Rauyaree et al., 2005; Sarmiento-Villamil et al., 2018). In homologous pathways of $N$. crassa and $A$. nidulans, a scaffold protein is required for insulation (Dettmann et al., 2014; Jonkers et al., 2014; Frawley et al., 2018) and homologs of this scaffold protein have been bioinformatically predicted in a subdivision of the Ascomycota (Jamet-Vierny et al., 2007). In this study, the impact of the corresponding V. dahliae protein Ham5 on Vmk1 MAPK pathway-mediated functions was analyzed.

Vmk1 has a conserved essential role in pathogenicity and MAPK deletion strains displayed reduced conidiation and microsclerotia formation (Rauyaree et al., 2005). A 
decrease in vegetative growth on certain media was observed in this study (Figure 13). The MAP2K Mek2 displayed the same impact on the phenotype as Vmk1 regarding microsclerotia formation, vegetative growth, and virulence (Figure 13, Figure 14). In addition to the high conservation of $V$. dahliae Mek2 to characterized homologs (Figure 12 ), these results provide indirect evidence that the kinase functions in the same MAPK pathway like Vmk1. No hints for additional targets of Mek2 have been observed. V. dahliae Vph1 was suggested to act downstream of the cascade as a yeast Ste12-like transcription factor activated upon phosphorylation by Vmk1 (Sarmiento-Villamil et al., 2018). The Vph1-deficient mutant displayed no alterations in its ex planta phenotype, but did not induce plant disease symptoms, suggesting that Vph1 is not the only target of the Vmk1 MAPK pathway in V. dahliae (Sarmiento-Villamil et al., 2018). This study showed that Vmk1 cascade-mediated functions in growth, microsclerotia formation, and virulence are independent from the presence of Ham5 (Figure 13, Figure 14), corroborating that these functions do not rely on insulation by the potential scaffold protein in $V$. dahliae. In $N$. crassa and $A$. nidulans the Ham5 scaffold protein homologs display similar functions as the Fus3/Kss1-like MAPK (Li et al., 2005; Aldabbous et al., 2010; Frawley et al., 2018). For example, N. crassa HAM-5 and MAK-2 are essential for hyphal and germling fusion, have a positive impact on vegetative growth, formation of aerial hyphae, as well as on sexual and asexual development (Li et al., 2005; Aldabbous et al., 2010). Similarly, the homolog IDC1 displays diverse impacts on fungal growth and differentiation in $P$. anserina. IDC1 is involved in the non-self-perception resulting in the reduction of vegetative growth and the induction of melanization in the stationary phase (JametVierny et al., 2007). In P. anserine, IDC1 contributes to sexual and asexual development and aerial hyphae formation (Jamet-Vierny et al., 2007). The observed phenotypes in absence of IDC1 display high similarity to phenotypes observed in absence of components of the cell wall integrity (CWI) MAPK pathway and migration of the respective MAPK to the nucleus is dependent on IDC1 in this fungus. These results led to the positioning of $P$. anserina IDC1 into another signaling cascade than its homologs in A. nidulans and N. crassa (Jamet-Vierny et al., 2007).

A scaffolding function of $V$. dahliae Ham5 in the CWI MAPK pathway, as suggested in $P$. anserina, is possible, but rather unlikely, since one would expect the observation of an ex planta or in planta phenotype in absence of a CWI MAPK cascade scaffold protein for the tested conditions. Even if homologs of the CWI MAPK pathway components in $V$. dahliae have not been characterized to date and their functions in fungal growth, differentiation, and virulence are still unknown, homologs of this cascade in related fungi possess effects on the response to cell wall stressors in many cases, and even more conserved, have strong impacts on virulence (Rui \& Hahn, 2007; Zhao \& Xu, 2007; 
Hamel et al., 2012; Segorbe et al., 2017; Jiang et al., 2018a). The cell wall stress response to SDS was unaffected in the absence of Ham5 in Verticillium and no impact on virulence was observed in this study. Overlapping functions of the Vmk1 and CWI MAPK pathways regarding their function in microsclerotia production are possible. The $V$. dahliae MAP2K and the MAPK homologs of the CWI pathway could be identified by BLAST searches due to their high conservation and were found to be upregulated in later stages of microsclerotia formation similar to Vmk1 and Mek2 (Xiong et al., 2014). This could be a hint for an impact of the CWI MAPK pathway on microsclerotia production or melanization, which would be expected to be reflected in the deletion phenotype of a scaffold protein as well.

Similar to the CWI MAPK pathway, a scaffold function of Ham5 for the Hog1 MAPK pathway is unlikely. The response to osmotic stress was not affected in absence of Ham5 in V. dahliae ( $\mathrm{NaCl}, \mathrm{H}_{2} \mathrm{O}_{2}$, Sorbitol). Besides their role in the response to osmotic stressors, the Hog1 MAPK pathway cytosolic osmo-sensor VdSsk1, the MAP2K VdPbs2, and the MAPK VdHog1 display partially overlapping functions with the Vmk1 MAPK pathway with positive impacts on microsclerotia formation or melanization and virulence (Wang et al., 2016; Tian et al., 2016; Zheng et al., 2019; Figure 31).

Shared functions with the cascade components could be shown also for potential receptors of the Vmk1 and Hog1 MAPK pathway. Direct evidence for upstream regulators for the activation of the Vmk1 MAPK pathway has not been given to date, but regulatory impacts of two transmembrane receptors, VdMsb2 and VdSho1, on the Vmk1 MAPK pathway in $V$. dahliae would be possible (Figure 31). Both receptors have been characterized regarding their functions in development and virulence in $V$. dahliae and display positive impacts on conidiation and virulence (Tian et al., 2014; Qi et al., 2016), similar to Vmk1 (Rauyaree et al., 2005). Microsclerotia development and melanization were unaffected in absence of VdSho1 (Qi et al., 2016), whereas microsclerotia production was reduced in a VdMsb2 deletion strain (Tian et al., 2014) similar to Vmk1 and Mek2 deletion strains in this study. Further, the receptors are involved in oxidative stress responses (Tian et al., 2014; Qi et al., 2016). Hints from studies in other fungi are given, which support the assumption that the receptors could have regulatory roles for both, Vmk1 and Hog1 MAPK pathways in V. dahliae. In yeast the transmembrane proteins Msb2 and Sho1 are involved in the activation of both, the Kss1 and the Hog1 MAPK pathway (Cullen et al., 2012). Physical interaction of Sho1 with the Fus3/Kss1-like MAPK module could be shown in F. graminearum (Gu et al., 2015). In F. oxysporum, impacts of the receptors on phosphorylation of the Fus3/Kss1-like MAPK homolog, expression of corresponding target genes, as well as on invasive growth were observed (Perez-Nadales \& Di Pietro, 2015). However, Msb2 and Sho1 influence the MAPK- 
dependent functions only partially, corroborating the presence of additional regulatory components for this pathway in F. oxysporum (Perez-Nadales \& Di Pietro, 2015).

High osmolarity

glycerol pathway
Vmk1

MAPK pathway
Cell wall

integrity pathway

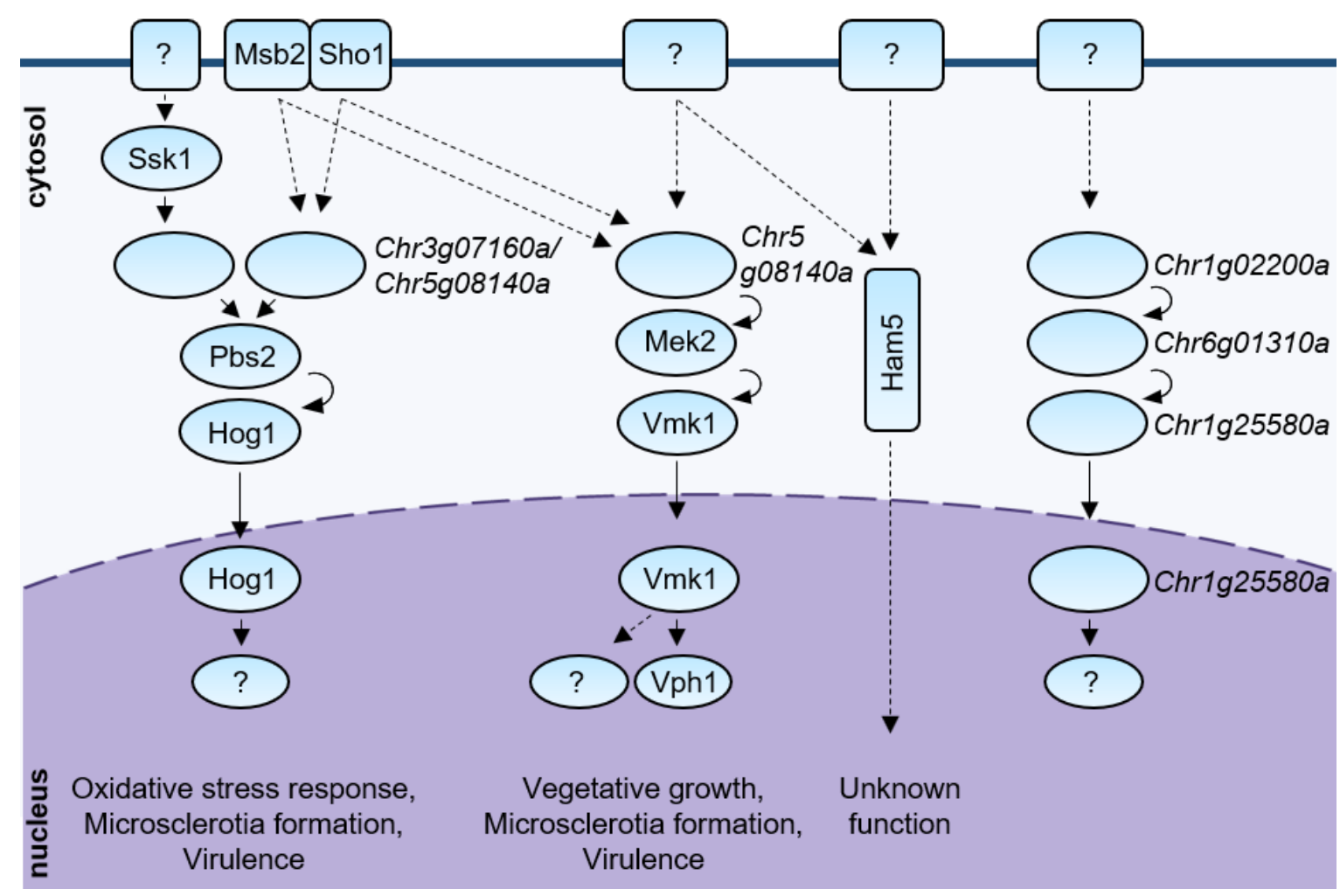

Figure 31: MAPK signaling pathways in $V$. dahliae. In $V$. dahliae components of two MAPK cascades, the Vmk1 and the Hog1 MAPK signaling pathways, were described to date. In this study, the MAP2K Mek2 and the Ham5 homolog of the Vmk1 MAPK cascade were characterized. Ham5 is dispensable for growth, microsclerotia formation, and virulence and therefore cannot be assumed to play a scaffolding role for these functions of the cascade. The functions of Ham5 in V. dahliae is unknown. Mek2 displays Vmk1-like positive regulatory roles in vegetative growth, microsclerotia production, and virulence. Indirect evidence was shown for Vph1 to act as a downstream transcription factor homologous to yeast Ste12. Vph1 functions in regulation of virulence but was dispensable for growth and microsclerotia production (Sarmiento-Villamil et al., 2018). Therefore, the presence of other potential targets of Vmk1 can be assumed. The Hog1 MAPK pathway displays similar functions in microsclerotia formation like the Vmk1 MAPK pathway. Both pathways are involved in regulation of virulence, however, the Vmk1 MAPK pathway has a greater impact. Described components are given with their respective names as determined in VdLs.17 (Xiong et al., 2014), but to date unstudied components are indicated with the V. dahliae JR2 gene identifiers (gene identifiers abbreviated for a better overview, VDAG_JR2_Chr...). The cell wall integrity MAPK pathway was not yet studied. Dashed lines indicate potential interactions. Question marks indicate, that proteins are unknown.

Structural differences between $V$. dahliae Ham5 to homologs in $N$. crassa and A. nidulans could be a reason for the lacking functional conservation. The characterized HAM5 gene in $V$. dahliae was the only hit during reciprocal BLAST search with known 
homologs and the deduced protein possesses a similar domain structure and higher protein sequence identity to $N$. crassa and all other species tested in this study than the A. nidulans homolog (Figure 11). However, V. dahliae Ham5 contains a shorter WD40 domain with 255 aa in comparison to 335 aa for N. crassa (Jonkers et al., 2016) and 311 aa for $A$. nidulans (Frawley et al., 2018). In N. crassa the N-terminal WD40 domain of HAM-5 was described to be essential for its function and the interaction with the MAK-2 protein, whereas the C-terminal domain plays a minor role for its function (Jonkers et al., 2014). WD40 repeat domains generally display roles in multi-protein interactions (Xu \& Min, 2011) and have been identified in several fungal scaffold proteins (Pöggeler \& Kück, 2004; Jain, 2019; Hicks et al., 2001). The N-terminal truncation in $V$. dahliae might have an impact on its function in mediating protein-protein interactions. In conclusion, a scaffolding function of the $V$. dahliae Ham5 homolog for one of the MAPK pathways in $V$. dahliae is rather unlikely, but might be possible in a not yet identified context. Further the Ham5 protein could be required for protein-protein interactions in another cascade (Figure 31). To date the function of the Ham5 protein in $V$. dahliae remains unknown. Even if the tested functions of the Vmk1- and Mek2mediated MAPK pathway are independent from the Ham5 homolog in $V$. dahliae, it is possible that a non-homologous scaffold protein, different to Ham5, might have insulating function on the cascade.

\subsubsection{V. dahliae Mek2 and Vmk1 are involved in fungal propagation in planta}

In the course of this study, it could be shown that the V. dahliae MAPK pathway components Mek2 and Vmk1 display crucial roles in induction of disease symptoms in tomato plants (Figure 14A). Hypocotyl discolorations as a hint for the induction of plant defense responses have not been observed following treatment with MEK2 or VMK1 deletion strains. These results were similar to findings of two $V$. dahliae Vmk1-deficient isolates from lettuce and tomato with severely reduced virulence and absence of vascular discolorations in tomato, cotton, egg-plant, and watermelon (Rauyaree et al., 2005). During both studies, plant experiments were performed with wounded plant roots, since Verticillia colonize plants preferentially via root wounds (Pegg \& Brady, 2002; Su et al., 2018). At this point, the role of Mek2 and Vmk1 in initial plant invasion of the root surface is unknown, but could be tested in root colonization assays. Plant infection experiments with the potential downstream Ste12-like transcription factor Vph1 suggested an essential role of the protein in hyphopodia formation and penetration of the root cortex (Sarmiento-Villamil et al., 2018). Only indirect evidence was given that Vph1 is a target of the Vmk1 MAPK pathway in V. dahliae (Sarmiento-Villamil et al., 2018). 
This study shows that the Fus3/Kss1-like MAPK pathway components Vmk1 and Mek2 contribute to, but are not essential for fungal propagation inside the plant tissue. $\triangle M E K 2$, $\triangle V M K 1, \triangle H A M 5 \triangle M E K 2$, or $\triangle H A M 5 \triangle V M K 1$ strains were successfully re-isolated from stem sections of infected plants, although to a minor extend compared to wild type or $\triangle H A M 5$ and, therefore, must be able to survive within the plant (Figure 14B, C). Unlike Vmk1 and Mek2 in this study, mutants deficient in Vph1 could not be re-isolated from asymptomatic plants (Sarmiento-Villamil et al., 2018). This might be due to different experimental settings in the respective plant infection experiments, since Vph1 deletion strain-treated plants were not wounded prior to spore inoculation different to Vmk1 and Mek2-treated plants in this study.

In several phytopathogenic fungi, the Fus3/Kss1-like MAPK deletion strains were apathogenic and the MAPK was not only essential for penetration of plant surfaces, but also essential for invasive growth and propagation within wounded plants (Xu \& Hamer, 1996; Takano et al., 2000; Zheng et al., 2000; Ruiz-Roldán et al., 2001; Mey et al., 2002). In others, the absence of the MAPK resulted in severely reduced virulence, invasive growth, and propagation, but not in the complete disability of fungal propagation within the host plant as in V. dahliae Vmk1-and Mek2-deficient strains in this study (Lev et al., 1999; Di Pietro et al., 2001; Cho et al., 2007). As an example, the A. brassicicola Fus3/Kss1-like MAPK Amk1 is essential for penetration of plant surfaces and the amk1 mutant was avirulent on intact host tissue, whereas it was able to colonize and partially induce disease symptoms on damaged host surfaces (Cho et al., 2007). The supplementation of damaged plant leaf material was sufficient to induce disease symptoms even on intact leaf surfaces, leading to the assumption that biochemical signals from damaged tissue were required to induce infection-related development in the amk1 mutant (Cho et al., 2007). The inability to penetrate intact host surfaces was correlated with decreased hydrolytic enzyme gene expression levels in absence of Amk1, which was increased on wounded tissue (Cho et al., 2007). A role of the Fus3/Kss1-like MAPK in regulation of cell wall degrading enzymes was observed as well in other phytopathogens as, for example, in F. oxysporum, Fusarium graminearum, and Cochliobolus heterostrophus (Di Pietro et al., 2001; Lev \& Horwitz, 2003; Jenczmionka \& Schäfer, 2005; Jiang et al., 2018a). In C. heterostrophus, cellulase genes were regulated by the MAPK pathway (Lev \& Horwitz, 2003). The F. oxysporum MAPK displayed an impact on gene expression of pectate lyases (Di Pietro et al., 2001), whereas the MAPK was dispensable for the production of pectinolytic or amylolytic enzymes in F. graminearum and was responsible for lipolytic activities instead (Jenczmionka \& Schäfer, 2005). 
In this study, the regulation of pectinolytic enzymes was not noticeably altered in absence of Vmk1 and Mek2 and seems to be mainly independent of the respective MAPK pathway in V. dahliae. The colony size of Mek2- and Vmk1-deficient strains was unaffected on simulated xylem medium containing pectin as carbon source (Figure 13A). The Vmk1- and Mek2-mediated MAPK pathway probably does not play a major role in the regulation of cellulase genes in $V$. dahliae, since colonies formed on minimal medium with either sucrose or cellulose as carbon source display only slight decreases in vegetative growth in comparison to wild type and the relative difference is the same on both tested media (Figure 13).

In conclusion, it was shown that the $V$. dahliae Fus3/Kss1-like MAPK components Mek2 and Vmk1 contribute to, but are not essential for survival of the fungus within wounded host plants, whereas both proteins are crucial for induction of disease symptoms. It can be assumed that the MAPK pathway has additional functions in virulence, besides substrate degradation, early infection-related morphogenesis, and fungal propagation in the host plant in V. dahliae.

\subsection{The unfolded protein response regulator Hac1 mediates differentiation and virulence in $V$. dahliae}

\subsubsection{Unconventional splicing and translation of the bZIP transcription factor Hac1 is conserved in V. dahliae}

During the different stages of the fungal perception and colonization of a host, signaling cascades coordinate the expression and secretion of tools enabling the interaction. This requires unfolded protein response-mediated adaptation of the protein processing and secretion capacity to changing demands in the ER (Richie et al., 2011; Heimel et al., 2013). In yeast, the ER transmembrane sensor Ire1 processes cytosolic unconventional splicing of the bZIP transcription factor Hac1 encoding mRNA (Shamu \& Walter, 1996; Welihinda \& Kaufman, 1996; Sidrauski \& Walter, 1997; Gonzalez et al., 1999; Okamura et al., 2000; Figure 4). After splicing, the mRNA is translated into a protein that migrates to the nucleus and regulates transcription of UPR target genes (Mori et al., 1996, 1998). The impacts of the homologous transcription factor Hac1 on growth, differentiation, ER stress response, and virulence in $V$. dahliae were characterized in this study.

The Ire1-dependent splicing of the HAC1 mRNA is conserved in V. dahliae, since the HAC1 mRNA contains an unconventional intron with a high sequence and structural similarity to Ire1 spliced introns in other organisms (Figure 16A; Yoshida et al., 2001; Saloheimo et al., 2003; Mulder et al., 2004; Wimalasena et al., 2008; Richie et al., 2009; Cheon et al., 2011; Hooks \& Griffiths-Jones, 2011; Joubert et al., 2011; Heimel et al., 
2013; Montenegro-Montero et al., 2015). The intron matches the consensus sequence of the Ire1 targeted splice sequence in HAC1 homologs (Hooks \& Griffiths-Jones, 2011) and formation of the characteristic twin stem-loop structure, required for recognition of the cleavage sites by Ire1, was proposed (Sidrauski \& Walter, 1997; Figure 16B, C). The unconventionally spliced mRNA variant of HAC1 could be amplified from ER stress inducing growth conditions but as well from growth conditions without additional supplementation of ER stressors, suggesting that the HAC1 mRNA is unconventionally spliced to a certain extend also in absence of those ER stressors.

The Hac1 protein was shown to be translated in the HAC1'-HA strain in immunoblots (Figure 18), which show a shift of the distinct band. A similar band shift has been observed in immunoblot analysis of the homologous $\mathrm{Cib} 1^{\text {s }}$ protein in U. maydis, which was proven to be the correct protein by mass spectrometry (personal communication apl. Prof. Kai Heimel). A possible explanation for the band shift is the formation of SDS stable secondary structures, leading to a slower migration through the gel.

In this study, a role of an uninduced protein variant $\mathrm{Hac} 1^{\mathrm{u}}$ was not found in V. dahliae, similar to other ascomycetes. Translation of the uninduced HAC1 mRNA into a Hac1u protein could not be detected in immunoblots, even though the gene was expressed (Figure 17). This could be due to a translational block or a strong instability of the Hac1u protein. The $H A C 1^{i}-H A$ strain, expressing the unconventionally spliced mRNA variant of HAC1 in absence of the unspliced HAC1 mRNA variant, was viable and complemented the observed deletion phenotypes regarding microsclerotia formation and induction of disease symptoms in planta. This suggests that the presence of $H A C 1^{u}$ has no significant role for these functions in $V$. dahliae. Theoretically, both mRNAs, $H A C 1^{u}$ and $H A C 1^{i}$, could be translated into proteins with an identical $\mathrm{N}$-terminus but unique $\mathrm{C}$-termini due to the frame shift of the ORF after unconventional splicing (Figure 16A). The bZIP domain and the NLS are located in the identical N-terminus. Therefore, both hypothetical proteins contain the bZIP transcription factor domain important for the formation of dimers, which can be either homo- or heterodimers, and both could be translocated into the nucleus. In higher eukaryotes the HAC1 homolog XBP1 is translated into both, the uninduced and the induced protein variants (Yoshida et al., 2006, 2009). Here, the bZIP domain mediates homo- as well as heterodimerization, resulting either in XBP1 ${ }^{\mathrm{u}}-\mathrm{XBP} 1^{\mathrm{u}}$, XBP14-XBP1, or XBP1-XBP1 dimers (Tirosh et al., 2006; Yoshida et al., 2006). Homodimers of the XBP1 protein act as positive UPR regulators by binding to UPR target genes in the nucleus (Yamamoto et al., 2007). Since XBP1" contains a nuclear export signal, homodimers of this protein reside in the cytosol and heterodimerization prevents nuclear localization of the induced protein variant and thereby acts as negative regulator of the UPR (Tirosh et al., 2006; Yoshida et al., 2006). In the basidiomycete 
U. maydis, regulatory roles of the unspliced mRNA of the HAC1 homolog cib $1^{u}$ and the protein Cib1 ${ }^{\mathrm{u}}$ were proposed (Heimel et al., 2013). Overexpression of the induced mRNA variant $c i b 1^{s}$ results in hyperactivation of the UPR and lethality as long as the unspliced mRNA is not present in the cell. Expression of cib1 ${ }^{u}$ is required for attenuation of the transcriptional UPR regulator in U. maydis (Heimel et al., 2013).

The length of the unconventional intron plays an important role in posttranscriptional regulation of the HAC1 mRNA. The similarity of the short $20 \mathrm{nt}$ unconventional intron in $V$. dahliae to related filamentous ascomycetes (Figure 16B) supports the assumption that the translation of the $H A C 1^{u}$ mRNA might be either blocked or the protein is very unstable due to similar mechanisms. In contrast to $S$. cerevisiae, base-pairing interaction between the unconventional introns and the 5'UTR for inhibition of ribosomal translation cannot be observed in the HAC1 mRNA of filamentous ascomycetes due to the shortened length (Mulder \& Nikolaev, 2009). Truncation of the 5'UTR length was described to result in translation of the HAC1 mRNA upon ER stress in different Aspergilli, T. reesei, and A. brassisicola (Saloheimo et al., 2003; Mulder et al., 2004; Joubert et al., 2011). Initially, an impact of upstream open reading frames (uORFs) on translational repression was assumed (Saloheimo et al., 2003; Mulder et al., 2004). Studies in $A$. niger revealed base-pairing of the 5'UTR of the HAC1 mRNA with an inverted repeat sequence as attenuation mechanism (Mulder \& Nikolaev, 2009; Heimel, 2015). Additionally to the translational block of the unspliced HAC1 mRNA, accelerated degradation of $\mathrm{Hac}^{u}$ proteins was observed in yeast (Di Santo et al., 2016). These findings in related fungi might explain the absence of $\mathrm{Hac}^{\mathrm{u}}{ }^{\mathrm{u}}$ in $\mathrm{V}$. dahliae.

\subsubsection{The unfolded protein response regulator Hac1 mediates species-specific differentiation in $V$. dahliae}

In this study, it was shown that $V$. dahliae HAC1 affects growth and fungal differentiation even in the absence of typical UPR stress inducing agents (Figure 19-21). V. dahliae $H A C 1$ is essential for the formation of microsclerotia as resting structures and has a positive impact on conidiation. The role of $H A C 1$ in fungal growth and differentiation in V. dahliae is partially conserved to other filamentous fungi, but specific impacts of HAC1 could be observed as well.

The protein sequence conservation of Hac1 between homologs from V. dahliae, yeast, the dimorphic basidiomycete U. maydis, ascomycetes, and human is rather low with the highest similarity of $55 \%$ between $V$. dahliae and T. reesei (Figure 17). The low sequence conservation of $\mathrm{Hac} 1$ in the highly conserved pathway might be a result of its function in the crosstalk to developmental programs (Heimel et al., 2013). In several studies, it could be shown that the impact of the Hac1 homologs on fungal growth and 
differentiation is highly adapted, since even closely related species show different phenotypes in absence of the UPR regulatory transcription factor (Heimel et al., 2013; Montenegro-Montero et al., 2015).

V. dahliae HAC1 is involved in the response to ER stress. The HAC1 deletion strain was generally impaired in vegetative growth and did not show a pronounced additional impairment in response to tunicamycin. However, the expression of the induced splice variant of HAC1 improved the ability to cope with the ER stress situation in $V$. dahliae, although the expression levels of the induced splice variant in $H A C 1^{i}-H A$ were more than a third lower than in the wild type (Figure 18-20). Under non-stress conditions, HAC1'-HA displayed reduced growth in comparison to wild type, which might be explained by differential regulation of genes supporting vegetative growth in correlation to a hyperactive UPR. Similar as in V. dahliae, genomic integration of the spliced HAC1 mRNA variant resulted in decreased growth in Aspergillus niger correlated with a downregulation of mechanisms for energy generation and cell development, whereas the genes for the protein secretion machinery were upregulated (Carvalho et al., 2012).

In U. maydis and C. neoformans the UPR has no impact on vegetative growth in absence of ER stress as in yeast (Nikawa et al., 1996; Kaufman, 1999; Cheon et al., 2011; Heimel et al., 2013). In contrast, similar to the observed conidiation defects in $V$. dahliae, the absence of HAC1 resulted in severe reduction in conidiation in $M$. oryzae (Tang et al., 2015), A. niger (Mulder \& Nikolaev, 2009), and A. brassicicola (Joubert et al., 2011).

Besides the similar impact on conidiation, an important role of Hac1 in vegetative growth in absence of ER stress could be shown for the same fungi as in V. dahliae (Mulder \& Nikolaev, 2009; Joubert et al., 2011; Tang et al., 2015). The impact of the UPR regulator on fungal growth and conidiation in the absence of ER stress in these species suggests a constitutive activation of the UPR leading to the regulation of certain target genes involved in growth and developmental processes (Heimel, 2015). This is confirmed by the finding that the induced mRNA variant could be amplified from cultures grown under non-stress inducing conditions in $V$. dahliae as mentioned previously. In A. fumigatus, a basal UPR activity under non-stress conditions has been observed as well (Feng et al., 2011). Comparison of the transcriptomes of a HAC1 deletion strain and the wild type, either under non-stress or ER stress conditions, revealed very different gene expression profiles in A. fumigatus, corroborating the possibility to adjust the expression of UPR target genes to certain stress levels (Feng et al., 2011).

Different to the ER stress-specific UPR activation or the constitutive activation of the UPR to a certain level, in a few examples, the UPR was activated under specific conditions. The Hac1 homolog is dispensable for unstressed vegetative growth, but 
required for growth at higher temperatures and on complex substrates in $A$. fumigatus (Richie et al., 2009). A similar observation was made in N. crassa, where the UPR regulator is required especially for growth on crystalline cellulose substrates (Montenegro-Montero et al., 2015). Here, an interplay of the sterol regulatory element binding protein (SREBP) pathway, which regulates the secretion of lignocellulolytic enzymes in filamentous fungi, and the UPR was suggested (Montenegro-Montero et al., 2015; Qin et al., 2017). The secretion of cellulases is suppressed in absence of the UPR regulator in N. crassa (Montenegro-Montero et al., 2015; Qin et al., 2017). An impact of the UPR on cellulose degradation and a correlation to secretion of cellulases has been observed as well in T. reesei and A. nidulans (Collén et al., 2005; Brown et al., 2013; Wang et al., 2014; Qin et al., 2017). A direct role in coupling of the conserved UPR to organism-specific developmental programs was found for the U.maydis UPR transcription factor Cib1 (Heimel et al., 2013). The Cib1 protein contains a C-terminal domain specifically required for its interaction with the developmental regulator Clampless 1 (Clp1) of the b mating type-dependent signaling pathway (Heimel et al., 2010 , 2013). The heterodimer formation stabilizes Clp1, is important to initiate fungal propagation after penetration, has a role in effector secretion, and is involved in regulation of virulence in the smut fungus (Heimel et al., 2013; Hampel et al., 2016). The b mating type-dependent signaling pathway initiates the morphological switch from yeast to filamentous growth and is specifically found in heterobasidiomycetes (Kothe, 1996). Hac1 proteins in ascomycetes might undergo completely different protein-protein interactions in frame of coupling the UPR to differentiation.

In this study, it could be shown that HAC1 is essential for the formation of microsclerotia, the melanized resting structures of $V$. dahliae (Figure 19, 20). HAC1 deletion strains were unable to form microsclerotia under any tested condition and the expression of the induced HAC1 mRNA variant in $H A C 1^{i}-H A$ resulted in increased microsclerotia formation, regardless of a decreased expression level in comparison to wild type. One suggestion to explain the essential role of $H A C 1$ in microsclerotia formation is that the UPR is a crucial checkpoint to induce resting structure formation following sensing of unfavorable conditions. Microsclerotia formation can be observed at temperatures below the growth optimum ( $20^{\circ} \mathrm{C}$; Soesanto \& Termorshuizen, 2001), under nutrient-limited conditions as on complex substrates or in dense cultures, as well as in the presence of several stressors including ER stress inducing agents. The mechanisms activating the formation of microsclerotia in $V$. dahliae are not yet understood, even if several candidates were shown to influence the production. Components of the cAMP signaling pathway, which is stimulated by perception of high-glucose conditions, have negative impacts on the formation of microsclerotia such as the catalytic subunit of the cAMP- 
dependent protein kinase A (Tzima et al., 2010, 2012). As previously described in this work, several MAPK signaling components show positive regulatory impacts on microsclerotia formation as for example Vmk1 and Mek2 (Figure 13; Rauyaree et al., 2005; Tian et al., 2014; Qi et al., 2016; Wang et al., 2016; Tian et al., 2016; Zheng et al., 2019). Other candidates are substantial for the melanization as for example Vayg1 (Verticillium homolog to Aspergillus yellowish green 1), which is required for dihydroxynaphthalene biosynthesis (Fan et al., 2017). Even if all these candidates have impacts on microsclerotia formation or melanization, examples for essential roles comparable to $H A C 1$ are rare. One example with an essential role in microsclerotia formation is the transcription factor Som1 (sorting mitochondrial 1), which is additionally involved in growth, conidiation, adhesion, and virulence of $V$. dahliae (Bui et al., 2019). Som1 was found to be required for regulation of adhesin encoding genes, such as the transcription activators of adhesion Vta2 and Vta3 which are regulators of flocculin genes involved in adhesion and microsclerotia formation (Tran et al., 2014; Bui et al., 2019). In S. cerevisiae, it could be shown that Hac1 is involved in the regulation of flocculin genes and interacts with the general control of amino acid biosynthesis (Herzog et al., 2013) and that ER stressed cells display slightly increased flocculation (Scrimale et al., 2009). Therefore, the absence of microsclerotia in Som1-and Hac1-deficient $V$. dahliae strains hints to a crosstalk of Som 1 and the UPR in the regulation of microsclerotia formation. In conclusion, the UPR regulator HAC1 is active to a certain extend under non-stress conditions. It is involved in the regulation of growth and sporulation and might be a checkpoint to sense and transduce the necessity of resting structure formation in V. dahliae (Figure 32).

\subsubsection{V. dahliae Hac1 is essential for fungal colonization of the host plant}

In this study, it could be shown that the V. dahliae UPR regulator HAC1 is involved in colonization of the root surface, but not required for the penetration and initial invasion of the root cortex (Figure 22). Further, HAC1 is involved in successful propagation within the host plant (Figure 23). Less fungal growth on the root surface was observed for the HAC1 deletion strain in comparison to wild type and the VTA2 deletion strain in root colonization experiments. This can be either caused by impaired growth or by an inability to adhere to the surface. Similar to Hac1, the transcription factor Som1 was found to be required for proliferation on the root surface correlated to its impact on adhesin gene expression (Bui et al., 2019). Therefore, not only the similar impact on microsclerotia formation, but also the potential role of HAC1 in adhesion could hint into direction of an interplay of the UPR and Som1. However, the impact of HAC1 on adhesion requires further investigation. Different to $H A C 1, S O M 1$ is essential for penetration of the root 
surface (Bui et al., 2019). However, target genes of Som1 were found to control different steps of plant root penetration and colonization including the transcription factors Vta2 and Vta3 (Bui et al., 2019).

Similar to $V$. dahliae, the initial penetration of the plant surface was unaffected in the appressorium-forming fungus $A$. brassicicola in absence of the UPR regulator AbHacA (Joubert et al., 2011). In the dimorphic fungus U. maydis the Hac1 orthologue is required to induce biotrophic growth within the plant after formation of appressoria and penetration of the plant surface (Heimel et al., 2010; Heimel et al., 2013). In contrast, UPR components are involved in the initial penetration of the plant surface by $M$. oryzae (Yi et al., 2009; Tang et al., 2015; Jiang et al., 2018b).

Since fungal re-isolation from stems was not possible for the $V$. dahliae HAC1 deletion strain in this study, one can assume that the fungus is hardly able to propagate in the vascular system and primarily remains in the roots or lower parts of the plant or does not survive after initial colonization of the root cortex. The absence of HAC1 resulted in severely reduced conidiation in simulated xylem medium (Figure 21), which might disable fungal spreading in the vascular system. Besides the decrease in conidiation, increased sensitivity to plant phytoalexins was observed in the A. brassicicola UPR regulator-deficient strain. An altered sensitivity to plant antimicrobial compounds remains to be elucidated in the $V$. dahliae HAC1 deletion strain, but could comprise an explanation for the observed in planta phenotype. Tomato plants treated with the HAC1 deletion strain displayed severely decreased disease symptoms, however, in $\sim 30 \%$ of the plants rather mild symptoms could be observed (Figure 23A). The induction of these symptoms could be a result from damage caused by the fungus or from the energy costs of successful immune defense responses in the plants. The impairment in adapting to increased protein secretion demands upon host colonization might also be an explanation for the less pathogenic outcome of the interaction of the HAC1 deletion strain with the host plant as previously suggested in studies with the plant and animal pathogens A. fumigatus, A. brassicicola, and U. maydis (Joubert et al., 2011; Richie et al., 2011; Heimel et al., 2013).

It is possible that the plant is able to activate defense responses and suppress fungal colonization of the HAC1 deletion strain due to a lack of fungal effector gene expression and secretion, which are required to modulate the plants immune system. Examples for regulation of fungal effector genes by the corresponding UPR transcription factor in the maize colonizer U. maydis are given (Hampel et al., 2016). Further, it is possible that $V$. dahliae Hac1 regulates virulence factors independent of ER stress induction, similar to the signal peptide peptidase Ssp1 in U. maydis (Pinter et al., 2019). Here, deletion of the 
Cib1-regulated gene ssp1 resulted in an avirulent in planta phenotype without impacts of ssp1 on vegetative growth or ER stress response (Pinter et al., 2019).

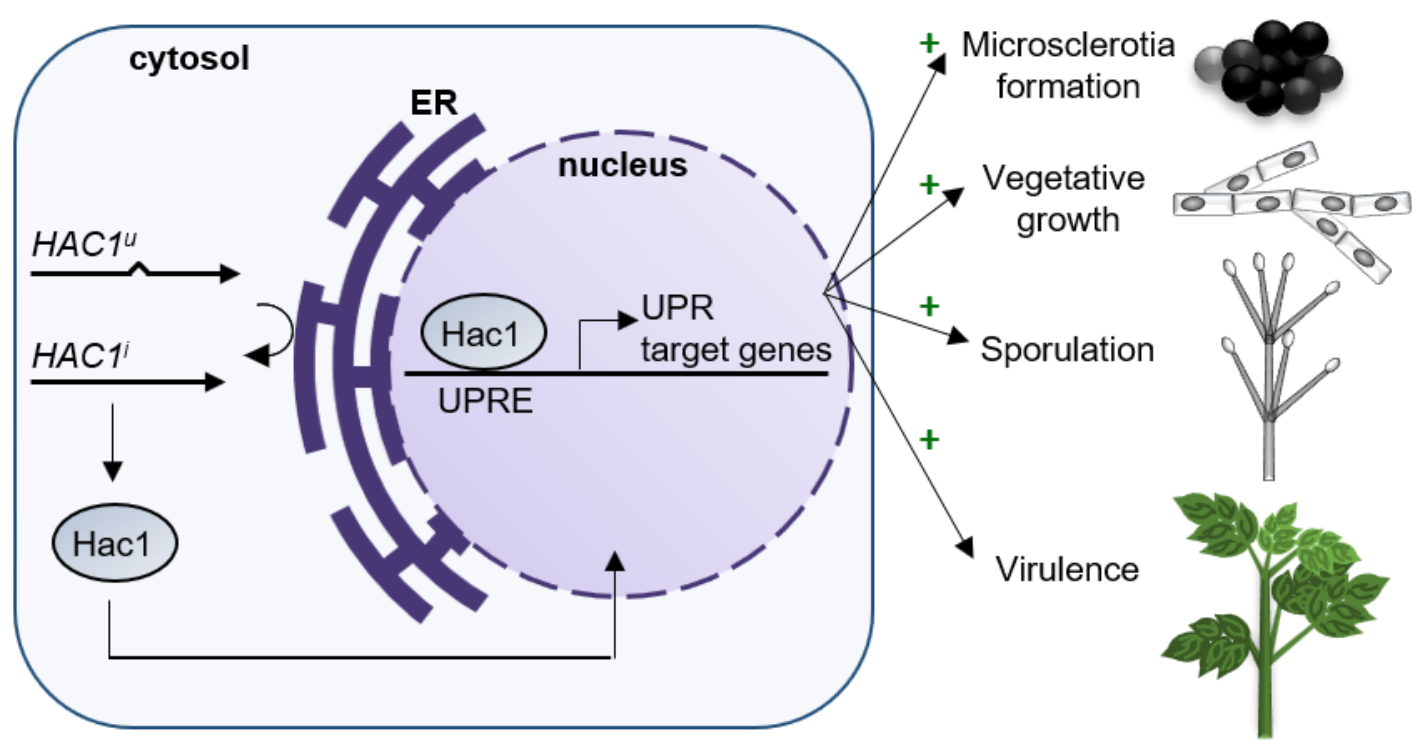

Figure 32: V. dahliae HAC1 regulates differentiation and virulence. The HAC1 mRNA occurs in two splice variants in the cell. The HAC1 ${ }^{u}$ mRNA contains an unconventionally spliced intron, which is presumably targeted by the ER transmembrane endoribonuclease Ire1 (indicated by an arrow located at the ER membrane). Splicing of the unconventional intron results in the mRNA splice variant $H A C 1^{i}$, which is translated into the protein Hac1. The transcription factor Hac1 presumably regulates transcription of UPR target genes by binding to UPR elements (UPRE) in their promoters and is essential for the development of microsclerotia as resting structures. Hac1 regulates vegetative growth under non-stress and ER stress conditions. Furthermore, HAC1 or Hac1-regulated genes are important for sporulation, fungal propagation within host plants, and induction of disease symptoms.

In this study, the impact of the constitutive presence of unconventionally spliced $H A C 1^{i}$ mRNA on growth, differentiation, and virulence of $V$. dahliae was tested by construction of $H A C 1^{i}-H A$. Ectopic integration into the HAC1 deletion strain resulted in decreased gene expression in comparison to wild type similar to the strain containing an ectopic integration of the HAC1u mRNA, HAC1 ${ }^{u}-H A$ (Figure 18). The decreased expression levels were sufficient to complement the microsclerotia and growth phenotype of the $H A C 1$ deletion strain in $H A C 1^{u}-H A$. For $H A C 1^{i}-H A$ even increased microsclerotia formation could be observed. The decreased growth in $H A C 1^{i}-H A$ is assumed to be caused by a differential gene expression of metabolic genes in response to the perception of ER stress similar to A. niger (Carvalho et al., 2012) and not due to an incomplete complementation. However, tomato plants treated with $H A C 1^{4}-H A$ or $H A C 1^{i}-H A$ strains displayed a similar induction of disease symptoms with intermediate severity between wild type and $H A C 1$ deletion strain infected plants, suggesting an impact of the decreased expression levels of $H A C 1$ on fungal virulence. Since the differences in HAC1 gene expression to wild type are similar in both strains and both 
strains induce similar severities of disease symptoms, an impact of the constitutive presence of unconventionally spliced HAC1 mRNA on virulence cannot be assumed.

In conclusion, the UPR regulator Hac1 plays a major impact on the fungal ability to colonize host plants, either due to decreased growth and fungal propagation on the root surface as well as decreased conidiation and spreading within the plant, but likely as well due to the inability to evade the plant immune response, since it is not required for penetration, but for propagation inside the host (Figure 32).

\subsection{The $V$. dahliae oleate $\Delta 12$-fatty acid desaturase Ode1 has important roles in fungal growth and is dispensable for induction of disease symptoms}

\subsubsection{The $V$. dahliae oleate $\Delta 12$-fatty acid desaturase Ode1 contributes to fungal growth and differentiation}

Different signaling molecules contribute to the crosstalk between fungi and their host plants and decide about the outcome of the interaction. Besides the secretion of effector proteins, which can be regulated by the UPR (Hampel et al., 2016; Pinter et al., 2019), lipid metabolites can act as signaling molecules (Christensen \& Kolomiets, 2011). Since linoleic acid and the derived oxylipins were described to be able to modulate fungal differentiation and the development of disease symptoms (Calvo et al., 2001; Brodhagen et al., 2008; Brodhun et al., 2009; Reverberi et al., 2010; Scala et al., 2014; Fischer \& Keller, 2016; Patkar \& Naqvi, 2017), the impact of the oleate $\Delta 12$-fatty acid desaturase Ode1 on differentiation and virulence of $V$. dahliae was analyzed.

Due to the protein domain structure, the similarity to the corresponding desaturase in A. nidulans, and the positive impact of medium supplementation with linoleic acid on growth of the ODE1 deletion strain (Figure 24, 26), V. dahliae Ode1 can be assumed to catalyze the conversion of oleic acid to linoleic acid. In $A$. nidulans and $A$. parasiticus, a depletion of linoleic acid and the derived products as well as an increase in oleic acid following disruption of the homologous ODE1 gene were observed (Calvo et al., 2001; Chang et al., 2004).

The construction of strains with Ode1 either N- or C-terminally fused to GFP has shown, that the $\mathrm{N}$-terminus of the protein is important for its function. C-terminally GFP tagged Ode1 is more stable than N-terminally GFP tagged Ode1 since diffuse GFP signals in vacuoles were observed for GFP-ODE1, which were absent in ODE1-GFP (Figure 25C). The vacuolar localization of diffuse GFP suggest an accelerated degradation of the membrane protein by autophagosomes in the vacuoles (Liu et al., 2012). The presence of an additional larger protein version of Ode1, specifically present for ODE1-GFP in 
immunoblots (Figure 25A), supports possible posttranslational modification of Ode1 as part of the mechanism controlling Ode1 activity.

V. dahliae Ode1 localizes to membranes of cell organelles, presumably ER membranes, and in irregular patterns in the plasma membranes, often close to growing hyphal tips or branch points (Figure 25B, C). The protein sequence predicted that Ode1 is a transmembrane protein, which is a typical feature of desaturases (Uttaro, 2006). A signal peptide sequence predicting ER localization was not identified, however $\Delta 12$-desaturases in plants, animals, and lower eukaryotes are generally described to be located either in plastids or in ER membranes (Uttaro, 2006). Even if few fungal $\Delta 12$-desaturases have been described, their localization has not yet been shown (Passorn et al., 1999; Sakuradani et al., 1999; Calvo et al., 2001; Watanabe et al., 2004; Chang et al., 2004; Wilson et al., 2004; Wei et al., 2006). The detection of Ode1 at sites of hyphal growth and branch points suggests a directed vesicle transport. The product of the fatty acid desaturase is linoleic acid, which is an important component of fungal plasma membranes with roles in adjusting membrane rigidity (Gostinčar et al., 2009). The presence of the desaturase close to hyphal tips could facilitate growth by serving one of the products required for extension of hyphae. During growth, liquid uptake supports the increase of the cell volume and exocytosis expands the plasma membrane and cell wall (Money, 2016). In related fungi such as Aspergilli and N. crassa, growth at hyphal tips or branch points was dependent on a structure close to these locations, named the Spitzenkörper as vesicle supply center, consisting of cytoskeletal elements and vesicles which are distributed to extend the hyphal tips by exocytosis (Harris et al., 2005). In N. crassa, chitin and glucan synthases localize to the Spitzenkörper to build up the cell wall (Su et al., 2012). Besides, linoleic acid might not only provide a building block for the membrane. On the other hand, it could increase the membrane fluidity and facilitate exocytosis. In yeast, it has been suggested that increased ergosterol contents in the membrane promote vesicle fusion (Munn et al., 1999).

Even if $V$. dahliae contains more than one gene encoding potential oleate $\Delta 12$-fatty acid desaturases in its genome, this study could show that Ode1 provides an important contribution to fungal growth and differentiation (Figure 33). This observations suggests that not all encoded $\Delta 12$-fatty acid desaturases might have additive or only partiallyredundant functions. Reciprocal BLAST search has unraveled the presence of a second potential homolog to $A$. nidulans odeA with only slightly lower conservation of the deduced amino acid sequence than Ode1, almost the same size (479 aa), and similar domain structure (VDAG_JR2_Chr2g10170a; FAD domains: 75-114 aa IPR021863, 142427 aa IPR005804; four transmembrane helices, Phobius). 
Besides, another gene is predicted to encode an OdeA non-homologous $\Delta 12$-fatty acid desaturase with 402 aa in length and different domain structure, which was clearly predicted to be localized to the ER membrane using DeepLoc-1.0 and with its $\mathrm{N}$-terminus directed to the ER lumen (VDAG_JR2_Chr4g12440a; FAD domain: 85-367 aa IPR005804; N-terminus located in ER 1-53 aa, Phobius; four transmembrane helices, Phobius). Deletion of ODE1 as a single of these desaturases resulted in a decrease in the colony diameter about $50 \%$ in comparison to wild type. This was qualitatively similar on every tested medium including media supplemented with osmotic or cell wall stressors. The only more favorable condition was caused by substitution of linoleic acid (Figure 26).

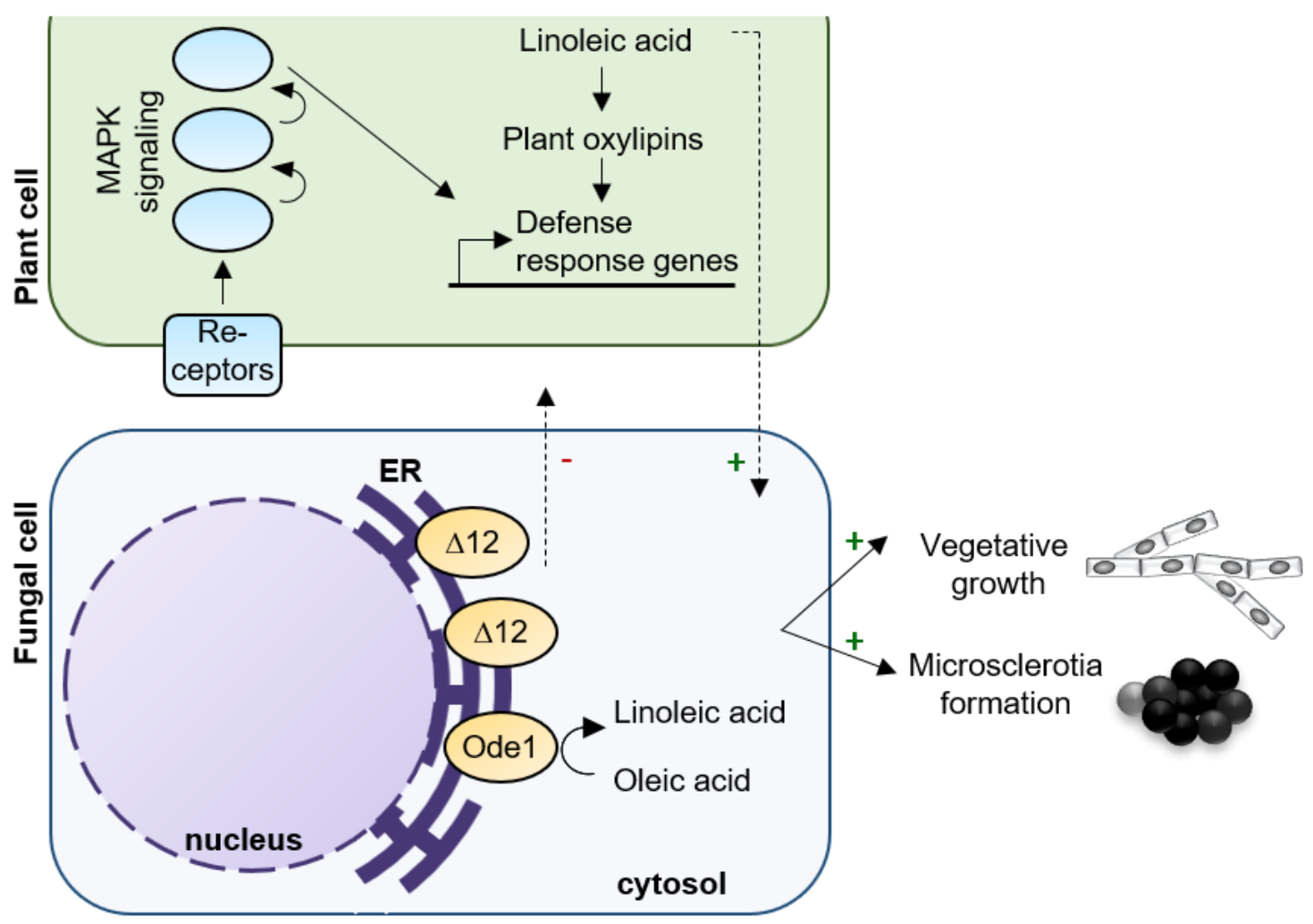

Figure 33: V. dahliae Ode1 displays a strong impact on vegetative growth, but is dispensable for induction of disease symptoms. The oleate $\Delta 12$-fatty acid desaturase Ode1 catalyzes the conversion of oleic acid into linoleic acid. The ODE1 defective strain displays impaired vegetative growth and microsclerotia formation, which is partially restored on medium supplemented with linoleic acid. This corroborates a positive impact of Ode1 or Ode1-derived products on growth and microsclerotia formation (indicated by arrows with green plus). The ODE1 deficiency displays only a minor impact on fungal virulence and the deletion strain is able to induce severe disease symptoms in planta. The availability of plantderived linoleic acid might complement the observed growth defect (indicated by arrow with dashed line and green plus) and promote fungal propagation or enable the biosynthesis of linoleic acid-derived products with unknown impact on the plants defense responses. Additional oleate $\Delta 12$-fatty acid desaturases $(\Delta 12)$ in $V$. dahliae could compensate the defect in $O D E 1$ and allow modulation of plant defense responses (indicated by arrows with dashed line with red minus). 
Similarly, a general decrease in growth was observed for $A$. nidulans and $A$. parasiticus upon deletion of the homologous oleate $\Delta 12$-fatty acid desaturases (Calvo et al., 2001; Chang et al., 2004; Wilson et al., 2004). BLAST and domain searches of $A$. nidulans OdeA revealed that the genome of this organism harbors an additional gene encoding a non-homologous protein with predicted $\Delta 12$-fatty acid desaturase domain and cytosolic $\mathrm{N}$-terminus (XP_664808.1) similar to the Ode1 non-homologous oleate $\Delta 12$-fatty acid desaturase predicted in $V$.dahliae, suggesting that also here other proteins with potentially overlapping functions exist.

With regard to the unaltered phenotype of the $V$. dahliae ODE1 deletion strain on stress media, one can conclude that Ode1 and its products are not especially required for the response to stresses even if one could expect that the adaptation of the membrane fluidity might influence stress responses. An increased membrane content of unsaturated fatty acids increased the sensitivity to salt stress in yeast for example (Gostinčar et al., 2009). It is possible that different desaturases are upregulated in response to certain environmental conditions or at certain developmental stages. FAD2, a single of a multitude of desaturases in $A$. thaliana, is activated upon sensing of ER stress and increases the ER stress tolerance by its positive impact on membrane fluidity (Nguyen et al., 2019).

Additionally to the impact on growth, $V$. dahliae Ode1 contributes to microsclerotia formation (Figure 27). Whereas here only a reduced resting structure formation was observed, deletion of the ODE1 homolog in A. parasiticus resulted in a complete loss of sclerotia development (Chang et al., 2004; Wilson et al., 2004). Since the ODE1 deletion strain displayed no specific stress-dependent phenotype one can assume that the decrease in microsclerotia formation can be seen as a result from the general growth defect and the altered polyunsaturated fatty acid metabolism.

\subsubsection{Plant-derived unsaturated fatty acids might replace products synthesized by $V$. dahliae Ode1 during host colonization}

V. dahliae Ode1 contributes to fungal growth even if the genome possesses additional genes, which are hypothetically encoding oleate $\Delta 12$-fatty acid desaturases. Ode 1 is not required for induction of severe disease symptoms in tomato plants as deletion strain infected plants display only a minor decrease in severity of disease symptoms (Figure 28). Therefore, Ode1 is not required for initial colonization and penetration. Inside the plant, the $O D E 1$ deletion strain might be able to use plant-derived unsaturated fatty acids to compensate for the growth defect. It was shown that the ODE1 deletion strain was able to use exogenous linoleic acid (Figure 26) and the plant provides linoleic acid which is used as a membrane component, precursor of plant oxylipins, and building blocks for 
the cuticula for example (Soliday \& Kolattukudy, 1977; Brodhun \& Feussner, 2011). Plant linoleic acid and the derived oxylipins are recognized as mimics of the fungal signaling molecules and promote sporulation and mycotoxin production in Aspergilli (Burow et al., 1997; Calvo et al., 1999; Wilson et al., 2004; Brodhagen et al., 2008; Gao \& Kolomiets, 2009; Horowitz Brown et al., 2009; Reverberi et al., 2010). In A. nidulans and A. flavus, perception of exogenous oxylipins via G-protein coupled receptors was confirmed for a few oxylipins (Affeldt et al., 2012, 2014; Fischer \& Keller, 2016).

Even if plants might provide substitutes for fungal linoleic acid or its derived compounds, it cannot be excluded that products from other oleate $\Delta 12$-fatty acid desaturases contribute to successful host colonization in $V$. dahliae. Endogenous linoleic acid production of the corresponding $A$. parasiticus oleate $\Delta 12$-fatty acid desaturase OdeA has positive effects on the colonization of live corn and peanut seeds, even if those provide linoleic acid contents (Wilson et al., 2004). The absence of odeA did not alter fungal growth on dead seeds. Therefore, fungal linoleic acid or linoleic acid-derived products were assumed to be required for suppression of defense responses in the seed (Wilson et al., 2004). The expression of plant LOX genes was modulated upon colonization by Aspergilli, resulting in altered jasmonic acid induced plant defense responses (Burow et al., 2000; Tsitsigiannis \& Keller, 2007; Horowitz Brown et al., 2009; Gao et al., 2009). Similar results with essential roles of oxylipins from both interaction partners have been found in F. verticilloides, where fungal oxylipins produced by LDS1 induce maize LOX3 for suppression of the plant JA-mediated defense responses and promote fungal virulence (Battilani et al., 2018).

In conclusion, $V$. dahliae Ode1 is dispensable for induction of severe disease symptoms even if the respective deletion strain displays a severe growth defect ex planta. Therefore, linoleic acid or linoleic acid derived compounds are either dispensable for virulence or the fungus can compensate the loss of Ode1-derived products through the action of other endogenous enzymes or exogenous plant-derived polyunsaturated fatty acids (Figure 33). 


\section{References}

Affeldt KJ, Brodhagen M \& Keller NP (2012) Aspergillus oxylipin signaling and quorum sensing pathways depend on $\mathrm{G}$ protein-coupled receptors. Toxins (Basel). 4: 695-717.

Affeldt KJ, Carrig J, Amare M \& Keller NP (2014) Global survey of canonical Aspergillus flavus G protein-coupled receptors. MBio 5: e01501-14.

Aldabbous MS, Roca MG, Stout A, Huang IC, Read ND \& Free SJ (2010) The ham-5, rcm-1 and rco-1 genes regulate hyphal fusion in Neurospora crassa. Microbiology 156: 2621-2629.

Ali GS \& Reddy A (2008) PAMP-triggered immunity: Early events in the activation of Flagellin Sensitive2. Plant Signal. Behav. 3: 423-426.

Almagro Armenteros JJ, Sønderby CK, Sønderby SK, Nielsen H \& Winther O (2017) DeepLoc: prediction of protein subcellular localization using deep learning. Bioinformatics 33: 33873395.

Andolfi A, Maddau L, Cimmino A, Linaldeddu BT, Basso S, Deidda A, Serra S \& Evidente A (2014) Lasiojasmonates A-C, three jasmonic acid esters produced by Lasiodiplodia sp., a grapevine pathogen. Phytochemistry 103: 145-153.

Andreou A \& Feussner I (2009) Lipoxygenases - Structure and reaction mechanism. Phytochemistry 70: 1504-1510.

Balnojan S (2016) Influence of the Fbx15 protein and adjacent elements on the development and virulence of Verticillium dahliae. Master thesis at the University of Goettingen.

Bardwell L, Cook JG, Voora D, Baggott DM, Martinez AR \& Thorner J (1998) Repression of yeast Ste12 transcription factor by direct binding of unphosphorylated Kss1 MAPK and its regulation by the Ste7 MEK. Genes Dev. 12: 2887-2898.

Battilani P, Lanubile A, Scala V, Reverberi M, Gregori R, Falavigna C, Dall'asta C, Park Y-S, Bennett J, Borrego EJ \& Kolomiets MV (2018) Oxylipins from both pathogen and host antagonize jasmonic acid-mediated defence via the 9-lipoxygenase pathway in Fusarium verticillioides infection of maize. Mol. Plant Pathol. 19: 2162-2176.

Bayram Ö, Sarikaya-Bayram Ö, Ahmed YL, Maruyama J, Valerius O, Rizzoli SO, Ficner R, Irniger S \& Braus GH (2012) The Aspergillus nidulans MAPK module AnSte11-Ste50-Ste7-Fus3 controls development and secondary metabolism. PLoS Genet. 8: e1002816.

Bergkessel M \& Guthrie C (2013) Colony PCR. In Laboratory Methods in Enzymology., Lorsch J (ed), San Diego, USA: Elsevier. 1: 299-309.

Berlanger I \& Powelson ML (2000) Verticillium wilt. Plant Heal. Instr. Am. Phytopathol. Soc. doi:10.1094/PHI-I-2000-0801-01. Updated 2005.

Bertani G (1951) Studies on lysogenesis I. The mode of phage liberation by lysogenic Escherichia coli. J. Bacteriol. 62: 293-300.

Bhat RG \& Subbarao KV (1999) Host range specificity in Verticillium dahliae. Genet. Resist. 89: 1218-1225.

Boyce KJ, Chang H, D'Souza CA \& Kronstad JW (2005) An Ustilago maydis septin is required for filamentous growth in culture and for full symptom development on maize. Eukaryot. Cell 4: 2044-2056.

Bradford MM (1976) A rapid and sensitive method for the quantitation of microgram quantities of protein utilizing the principle of protein-dye binding. Anal. Biochem. 72: 248-254.

Brasier C (2000) The rise of the hybrid fungi. Nature 405: 134-135.

Brodhagen M, Tsitsigiannis DI, Hornung E, Goebel C, Feussner I \& Keller NP (2008) Reciprocal oxylipin-mediated cross-talk in the Aspergillus-seed pathosystem. Mol. Microbiol. 67: 378391.

Brodhun F \& Feussner I (2011) Oxylipins in fungi. FEBS J. 278: 1047-1063.

Brodhun F, Go C, Hornung E \& Feussner I (2009) Identification of PpoA from Aspergillus nidulans as a fusion protein of a fatty acid heme dioxygenase/ peroxidase and a cytochrome P450. J. Biol. Chem. 284: 11792-11805. 
Brown N, de Gouvea P, Krohn N, Savoldi M \& Goldman G (2013) Functional characterisation of the non-essential protein kinases and phosphatases regulating Aspergillus nidulans hydrolytic enzyme production. Biotechnol. Biofuels 6: 91.

Brown NA, Urban M \& Hammond-Kosack KE (2016) The trans-kingdom identification of negative regulators of pathogen hypervirulence. FEMS Microbiol. Rev. 40: 19-40.

Bui (2017) Verticillium dahliae transcription factors Som1 and Vta3 control microsclerotia formation and sequential steps of plant root penetration and colonisation to induce disease. Dissertation at the University of Goettingen.

Bui T, Harting R, Braus-Stromeyer SA, Tran V, Leonard M, Höfer A, Abelmann A, Bakti F, Valerius O, Schlüter R, Stanley CE, Ambrósio A \& Braus GH (2019) Verticillium dahliae transcription factors Som1 and Vta3 control microsclerotia formation and sequential steps of plant root penetration and colonisation to induce disease. New Phytol. 221: 2138-2159.

Burow GB, Gardner HW \& Keller NP (2000) A peanut seed lipoxygenase responsive to Aspergillus colonization. Plant Mol. Biol. 42: 689-701.

Burow GB, Nesbitt TC, Dunlap J \& Keller NP (1997) Seed lipoxygenase products modulate Aspergillus mycotoxin biosynthesis. Mol. Plant-Microbe Interact. 10: 380-387.

Calvo AM, Gardner HW \& Keller NP (2001) Genetic connection between fatty acid metabolism and sporulation in Aspergillus nidulans. J Biol Chem. 276: 25766-25774.

Calvo AM, Hinze LL, Gardner HW \& Keller NP (1999) Sporogenic effect of polyunsaturated fatty acids on development of Aspergillus spp. Appl. Environ. Microbiol. 65: 3668-3673.

Caracuel Z, Roncero MIG, Espeso EA, González-Verdejo Cl, García-Maceira FI \& Di Pietro A (2003) The $\mathrm{pH}$ signalling transcription factor PacC controls virulence in the plant pathogen Fusarium oxysporum. Mol. Microbiol. 48: 765-779.

Carroll CL, Carter CA, Goodhue RE, Lawell C-YCL \& Subbarao KV (2018) A review of control options and externalities for Verticillium wilts. Phytopathology 108: 160-171.

Carvalho ND, Jørgensen TR, Arentshorst M, Nitsche BM, van den Hondel CA, Archer DB \& Ram AF (2012) Genome-wide expression analysis upon constitutive activation of the HacA bZIP transcription factor in Aspergillus niger reveals a coordinated cellular response to counteract ER stress. BMC Genomics 13: 350.

Castoria R, Fanelli C, Zoina A \& Scala F (1995) Analysis of fatty acids in lipids of Verticillium dahliae and induction of lubimin accumulation in eggplant. Plant Pathol. 44: 791-795.

Champe SP \& El-Zayat AA (1989) Isolation of a sexual sporulation hormone from Aspergillus nidulans. J. Bacteriol. 171: 3982-3988.

Chang P, Wilson RA, Keller NP \& Cleveland TE (2004) Deletion of the $\Delta 12$-oleic acid desaturase gene of a nonaflatoxigenic Aspergillus parasiticus field isolate affects conidiation and sclerotial development. J. Appl. Microbiol. 97: 1178-1184.

Chapman RE \& Walter P (1997) Translational attenuation mediated by an mRNA intron. Curr. Biol. 7: 850-859.

Chen C, Harel A, Gorovoits R, Yarden O \& Dickman MB (2004) MAPK regulation of sclerotial development in Sclerotinia sclerotiorum is linked with $\mathrm{pH}$ and cAMP sensing. Mol. PlantMicrobe Interact. 17: 404-413.

Chen CK-M, Chan N-L \& Wang AH-J (2011) The many blades of the $\beta$-propeller proteins: conserved but versatile. Trends Biochem. Sci. 36: 553-561.

Chen JY, Liu C, Gui YJ, Si KW, Zhang DD, Wang J, Short DPG, Huang JQ, Li NY, Liang Y, Zhang WQ, Yang L, Ma XF, Li TG, Zhou L, Wang BL, Bao YM, Subbarao KV, Zhang GY \& Dai XF (2018) Comparative genomics reveals cotton-specific virulence factors in flexible genomic regions in Verticillium dahliae and evidence of horizontal gene transfer from Fusarium. New Phytol. 217: 756-770.

Cheon SA, Jung KW, Chen YL, Heitman J, Bahn YS \& Kang HA (2011) Unique evolution of the UPR pathway with a novel bZIP transcription factor, Hxl1, for controlling pathogenicity of Cryptococcus neoformans. PLoS Pathog. 7: e1002177. 
Chini A, Cimmino A, Masi M, Reveglia P, Nocera P, Solano R \& Evidente A (2018) The fungal phytotoxin lasiojasmonate A activates the plant jasmonic acid pathway. J. Exp. Bot. 69: 30953102.

Cho Y, Cramer RA, Kim KH, Davis J, Mitchell TK, Figuli P, Pryor BM, Lemasters E \& Lawrence CB (2007) The Fus3/Kss1 MAP kinase homolog Amk1 regulates the expression of genes encoding hydrolytic enzymes in Alternaria brassicicola. Fungal Genet. Biol. 44: 543-553.

Cho Y, Srivastava A, Ohm RA, Lawrence CB \& Wang K-H (2012) Transcription factor Amr1 induces melanin biosynthesis and suppresses virulence in Alternaria brassicicola. PLoS Pathog 8: e1002974.

Choi KY, Satterberg B, Lyons DM \& Elion EA (1994) Ste5 tethers multiple protein kinases in the MAP kinase cascade required for mating in S. cerevisiae. Cell 78: 499-512.

Chou S, Lane S \& Liu H (2006) Regulation of mating and filamentation genes by two distinct Ste12 complexes in Saccharomyces cerevisiae. Mol Cell Biol. 13: 4794-4805.

Christensen MJ, Bennett RJ, Ansari HA, Koga H, Johnson RD, Bryan GT, Simpson WR, Koolaard JP, Nickless EM \& Voisey CR (2008) Epichloë endophytes grow by intercalary hyphal extension in elongating grass leaves. Fungal Genet. Biol. 45: 84-93.

Christensen SA \& Kolomiets MV (2011) The lipid language of plant-fungal interactions. Fungal Genet. Biol. 48: 4-14

Clewes E, Edwards SG \& Barbara DJ (2008) Direct molecular evidence supports long-spored microsclerotial isolates of Verticillium from crucifers being interspecific hybrids. Plant Pathol. 57: 1047-1057.

Coghlan A, Eichler EE, Oliver SG, Paterson AH \& Stein L (2005) Chromosome evolution in eukaryotes: a multi-kingdom perspective. Trends Genet. 21: 673-682.

Cole SJ, Yoon AJ, Faull KF \& Diener AC (2014) Host perception of jasmonates promotes infection by Fusarium oxysporum formae speciales that produce isoleucine- and leucineconjugated jasmonates. Mol. Plant Pathol. 15: 589-600.

Coleman JJ, Rounsley SD, Rodriguez-Carres M, Kuo A, Wasmann CC, Grimwood J, Schmutz J, Taga M, White GJ, Zhou S, Schwartz DC, Freitag M, Ma L, Danchin EGJ, Henrissat B, Coutinho PM, Nelson DR, Straney D, Napoli CA, Barker BM, et al. (2009) The genome of Nectria haematococca: contribution of supernumerary chromosomes to gene expansion. PLoS Genet. 5: e1000618.

Collado-Romero M, Jiménez-Díaz RM \& Mercado-Blanco J (2010) DNA sequence analysis of conserved genes reveals hybridization events that increase genetic diversity in Verticillium dahliae. Fungal Biol. 114: 209-218.

Collén A, Saloheimo M, Bailey M, Penttilä M \& Pakula TM (2005) Protein production and induction of the unfolded protein response in Trichoderma reesei strain Rut-C30 and its transformant expressing endoglucanase I with a hydrophobic tag. Biotechnol. Bioeng. 89: 335-344.

Collins A, Okoli CAN, Morton A, Parry D, Edwards SG \& Barbara DJ (2003) Isolates of Verticillium dahliae pathogenic to crucifers are of at least three distinct molecular types. Phytopathology 93: 364-376.

Conn PM (2011) The unfolded protein response and cellular stress. In Methods in Enzymology. Conn MP (ed). Los Angeles, CA, USA: Elsevier 489: 384.

Covert SF, Kapoor P, Lee M, Briley A \& Nairn CJ (2001) Agrobacterium tumefaciens-mediated transformation of Fusarium circinatum. Mycol. Res. 105: 259-264.

Cox JS, Shamu CE \& Walter P (1993) Transcriptional induction of genes encoding endoplasmic reticulum resident proteins requires a transmembrane protein kinase. Cell 73: 1197-206.

Cullen PJ, Sprague Jr. GF (2012) The regulation of filamentous growth in yeast. Genetics 190: 23-49.

Dagenais TRT, Chung D, Giles SS, Hull CM, Andes D \& Keller NP (2008) Defects in conidiophore development and conidium-macrophage interactions in a dioxygenase mutant of Aspergillus fumigatus. Infect. Immun. 76: 3214-3220. 
Dall'Asta C, Falavigna C, Galaverna G \& Battilani P (2012) Role of maize hybrids and their chemical composition in Fusarium infection and fumonisin production. J. Agric. Food Chem. 60: 3800-3808.

Dall'Asta C, Giorni P, Cirlini M, Reverberi M, Gregori R, Ludovici M, Camera E, Fanelli C, Battilani P \& Scala V (2015) Maize lipids play a pivotal role in the fumonisin accumulation. World Mycotoxin J. 8: 87-97.

David H, Ozçelik IS, Hofmann G \& Nielsen J (2008) Analysis of Aspergillus nidulans metabolism at the genome-scale. BMC Genomics 9: 163.

Debuchy R \& Turgeon BG (2006) Mating-type structure, evolution, and function in Euascomycetes. In The Mycota (A comprehensive treatise on fungi as experimental systems for basic and applied research). Kües U. FR (ed). Berlin, Heidelberg: Springer 1: 293-323.

de Jonge R, Bolton MD, Kombrink A, Van Den Berg GCM, Yadeta KA \& Thomma BPHJ (2013) Extensive chromosomal reshuffling drives evolution of virulence in an asexual pathogen. Genome Res. 23: 1271-1282.

de Jonge R, Peter van Esse H, Maruthachalam K, Bolton MD, Santhanam P, Saber MK, Zhang Z, Usami T, Lievens B, Subbarao KV \& Thomma BPHJ (2012) Tomato immune receptor Ve1 recognizes effector of multiple fungal pathogens uncovered by genome and RNA sequencing. Proc. Natl. Acad. Sci. 109: 5110-5115.

Depotter JRL, Deketelaere S, Inderbitzin P, von Tiedemann A, Höfte M, Subbarao KV, Wood TA \& Thomma BPHJ (2016a) Verticillium longisporum, the invisible threat to oilseed rape and other brassicaceous plant hosts. Mol. Plant Pathol. 17: 1004-1016.

Depotter JRL, Seidl MF, van den Berg GCM, Thomma BPHJ \& Wood TA (2017) A distinct and genetically diverse lineage of the hybrid fungal pathogen Verticillium longisporum population causes stem striping in British oilseed rape. Environ. Microbiol. 19: 3997-4009.

Depotter JRL, Seidl MF, Wood TA \& Thomma BPHJ (2016b) Interspecific hybridization impacts host range and pathogenicity of filamentous microbes. Curr. Opin. Microbiol. 32: 7-13.

Depotter JRL, Thomma BPHJ \& Wood TA (2019) Measuring the impact of Verticillium longisporum on oilseed rape (Brassica napus) yield in field trials in the United Kingdom. Eur. J. Plant Pathol. 153: 321-326.

Dettmann A, Heilig Y, Valerius O, Ludwig S \& Seiler S (2014) Fungal communication requires the MAK-2 pathway elements STE-20 and RAS-2, the NRC-1 adapter STE-50 and the MAP kinase scaffold HAM-5. PLoS Genet. 10: e1004762.

Di Santo R, Aboulhouda S \& Weinberg DE (2016) The fail-safe mechanism of post-transcriptional silencing of unspliced HAC1 mRNA. Elife 5: e20069.

Di Stasio M, Brefort T, Mendoza-Mendoza A, Münch K \& Kahmann R (2009) The dual specificity phosphatase Rok1 negatively regulates mating and pathogenicity in Ustilago maydis. Mol. Microbiol. 73: 73-88.

Djonovic S, Vargas WA, Kolomiets MV, Horndeski M, Wiest A \& Kenerley CM (2007) A proteinaceous elicitor Sm1 from the beneficial fungus Trichoderma virens is required for induced systemic resistance in maize. Plant Physiol. 145: 875-889.

Dong S, Raffaele S \& Kamoun S (2015) The two-speed genomes of filamentous pathogens: waltz with plants. Curr. Opin. Genet. Dev. 35: 57-65.

Eaton CJ, Cox MP, Ambrose B, Becker M, Hesse U, Schardl CL \& Scott B (2010) Disruption of signaling in a fungal-grass symbiosis leads to pathogenesis. Plant Physiol. 153: 1780-1794.

Edgar RC (2004) MUSCLE: multiple sequence alignment with high accuracy and high throughput. Nucleic Acids Res. 32: 1792-1797.

Ellenberger T, Fass D, Arnaud M \& Harrison SC (1994) Crystal structure of transcription factor E47: E-box recognition by a basic region helix-loop-helix dimer. Genes Dev. 8: 970-980.

Enebak SA, Haase DL, Pinto JR \& Riley LE (2012) Soil fumigation: the critical use exemption, quarantine pre-shipment rules, re-registration decision, and their effect on the 2012 growing season. Natl. Proc. For. Conserv. Nurs. Assoc. 68: 26-30. 
Evans JL, Moclock MA \& Gealt MA (1986) The fatty acid composition of the conidia and mycelia of the fungus Aspergillus nidulans. Can. J. Microbiol. 32: 179-181.

Eynck C, Koopmann B, Grunewaldt-Stoecker G, Karlovsky P \& von Tiedemann A (2007) Differential interactions of Verticillium longisporum and $V$. dahliae with Brassica napus detected with molecular and histological techniques. Eur. J. Plant Pathol. 118: 259-274.

Eynck C, Koopmann B \& von Tiedemann A (2009) Identification of Brassica accessions with enhanced resistance to Verticillium longisporum under controlled and field conditions. J. Plant Dis. Prot. 116: 63-72.

Faino L, Seidl MF, Shi-Kunne X, Pauper M, Van Den Berg GCM, Wittenberg AHJ \& Thomma BPHJ (2016) Transposons passively and actively contribute to evolution of the two-speed genome of a fungal pathogen. Genome Res. 26: 1091-1100.

Fan R, Klosterman SJ, Wang C, Subbarao K V, Xu X, Shang W \& Hu X (2017) Vayg1 is required for microsclerotium formation and melanin production in Verticillium dahliae. Fungal Genet. Biol. 98: 1-11.

Feng X, Krishnan K, Richie DL, Aimanianda V, Hartl L, Grahl N, Powers-Fletcher M V., Zhang M, Fuller KK, Nierman WC, Lu LJ, Latgé J-P, Woollett L, Newman SL, Cramer RA, Rhodes JC \& Askew DS (2011) HacA-independent functions of the ER stress sensor IreA synergize with the canonical UPR to influence virulence traits in Aspergillus fumigatus. PLoS Pathog. 7: e1002330.

Feys B (1994) Arabidopsis mutants selected for resistance to the phytotoxin coronatine are male sterile, insensitive to methyl jasmonate, and resistant to a bacterial pathogen. Plant Cell online 6: 751-759.

Fischer GJ \& Keller NP (2016) Production of cross-kingdom oxylipins by pathogenic fungi: An update on their role in development and pathogenicity. J. Microbiol. 54: 254-264.

Fitzell R, Evans G \& Fahy P (1980) Studies on the colonization of plant roots by Verticillium dahliae Klebahn with use of immunofluorescent staining. Aust. J. Bot. 28: 357-368.

Fleissner A, Leeder AC, Roca MG, Read ND \& Glass NL (2009) Oscillatory recruitment of signaling proteins to cell tips promotes coordinated behavior during cell fusion. Proc. Natl. Acad. Sci. U. S. A. 106: 19387-19392.

Floerl S, Druebert C, Majcherczyk A, Karlovsky P, Kües U \& Polle A (2008) Defence reactions in the apoplastic proteome of oilseed rape (Brassica napus var. napus) attenuate Verticillium longisporum growth but not disease symptoms. BMC Plant Biol. 8: 129.

Fogelqvist J, Tzelepis G, Bejai S, llbäck J, Schwelm A \& Dixelius C (2018) Analysis of the hybrid genomes of two field isolates of the soil-borne fungal species Verticillium longisporum. BMC Genomics 19: 14.

Fradin EF \& Thomma BPHJ (2006) Physiology and molecular aspects of Verticillium wilt diseases caused by $V$. dahliae and $V$. albo-atrum. Mol. Plant Pathol. 7: 71-86.

Fradin EF, Zhang Z, Juarez Ayala JC, Castroverde CD, Nazar RN, Robb J, Liu CM \& Thomma BPHJ (2009) Genetic dissection of Verticillium wilt resistance mediated by tomato Ve1. Plant Physiol. 150: 320-332.

Frawley D, Karahoda B, Sarikaya-Bayram Ö \& Bayram Ö (2018) The HamE scaffold positively regulates MpkB phosphorylation to promote development and secondary metabolism in Aspergillus nidulans. Sci. Rep. 8: 16588.

Fu C, lyer P, Herkal A, Abdullah J, Stout A \& Free SJ (2011) Identification and characterization of genes required for cell-to-cell fusion in Neurospora crassa. Eukaryot. Cell 10: 1100-1109.

Gao X, Brodhagen M, Isakeit T, Brown SH, Göbel C, Betran J, Feussner I, Keller NP \& Kolomiets MV (2009) Inactivation of the lipoxygenase $Z m L O X 3$ increases susceptibility of maize to Aspergillus spp. Mol. Plant-Microbe Interact. 22: 222-231.

Gao X \& Kolomiets MV (2009) Host-derived lipids and oxylipins are crucial signals in modulating mycotoxin production by fungi. Toxin Rev. 28: 79-88.

Gao X, Shim W-B, Göbel C, Kunze S, Feussner I, Meeley R, Balint-Kurti P \& Kolomiets MV (2007) Disruption of a maize 9-Lipoxygenase results in increased resistance to fungal 
pathogens and reduced levels of contamination with mycotoxin fumonisin. Mol. Plant-Microbe Interact. 20: 922-933.

Geer LY, Marchler-Bauer A, Geer RC, Han L, He J, He S, Liu C, Shi W \& Bryant SH (2010) The NCBI BioSystems database. Nucleic Acids Res. 38: S492-S496.

Gibriel HAY, Li J, Zhu L, Seidl MF \& Thomma BPHJ (2019) Verticillium dahliae strains that infect the same host plant display highly divergent effector catalogs. bioRxiv (ahead of print) doi:10.1101/528729.

Gonzalez TN, Sidrauski C, Dörfler S \& Walter P (1999) Mechanism of non-spliceosomal mRNA splicing in the unfolded protein response pathway. EMBO J. 18: 3119-3132.

Good M, Tang G, Singleton J, Reményi A \& Lim WA (2009) The Ste5 scaffold directs mating signaling by catalytically unlocking the Fus3 MAP kinase for activation. Cell 136: 1085-1097.

Goodrich-Tanrikulu M, Howe K, Stafford A \& Nelson MA (1998) Changes in fatty acid composition of Neurospora crassa accompany sexual development and ascospore germination. Microbiology 144: 1713-1720.

Gostinčar C, Turk M \& Gunde-Cimerman N (2009) Environmental impacts on fatty acid composition of fungal membranes. In Fungi from different environments, Misra JK \& Deshmukh SK (eds). Enfield, NH, USA: Science Publishers 1: 278-325.

Goto E, Ishido S, Sato Y, Ohgimoto S, Ohgimoto K, Nagano-Fujii M \& Hotta H (2003) c-MIR, a human E3 ubiquitin ligase, is a functional homolog of herpesvirus proteins MIR1 and MIR2 and has similar activity. J. Biol. Chem. 278: 14657-14668.

Griffiths DA (1970) The fine structure of developing microsclerotia of Verticillium dahliae Kleb. Arch. fuer Mikrobiol. 74: 207-212.

Gu Q, Chen Y, Liu Y, Zhang C \& Ma Z (2015) The transmembrane protein FgSho1 regulates fungal development and pathogenicity via the MAPK module Ste50-Ste11-Ste7 in Fusarium graminearum. New Phytol. 206: 315-328.

Guillemette T, Calmes B, Simoneau P \& Index FH (2014) Impact of the UPR on the virulence of the plant fungal pathogen $A$. brassicicola. Virulence 5: 357-364.

Guillemette T, Ram AF, Carvalho ND, Joubert A, Simoneau P \& Archer DB (2011) Methods for investigating the UPR in filamentous fungi. Methods Enzymol. 490: 1-29.

Hacquard S, Kracher B, Hiruma K, Münch PC, Garrido-Oter R, Thon MR, Weimann A, Damm U, Dallery J-F, Hainaut M, Henrissat B, Lespinet O, Sacristán S, Ver Loren van Themaat E, Kemen E, McHardy AC, Schulze-Lefert P \& O'Connell RJ (2016) Survival trade-offs in plant roots during colonization by closely related beneficial and pathogenic fungi. Nat. Commun. 7: $1-12$.

Hamel L-P, Nicole M-C, Duplessis S \& Ellis BE (2012) Mitogen-activated protein kinase signaling in plant-interacting fungi: distinct messages from conserved messengers. Plant Cell 24: 1327-1351.

Hampel M, Jakobi M, Schmitz L, Meyer U, Finkernagel F, Doehlemann G \& Heimel K (2016) Unfolded protein response (UPR) regulator Cib1 controls expression of genes encoding secreted virulence factors in Ustilago maydis. PLoS One 11: e0153861.

Hanahan D, Jessee J \& Bloom FR (1991) Plasmid transformation of Escherichia coli and other bacteria. Methods Enzymol. 204: 63-113.

Harris SD, Read ND, Roberson RW, Shaw B, Seiler S, Plamann M \& Momany M (2005) Polarisome meets Spitzenkörper: microscopy, genetics, and genomics converge. Eukaryot. Cell 4: 225-229.

Hartmann FE, Sánchez-Vallet A, McDonald BA \& Croll D (2017) A fungal wheat pathogen evolved host specialization by extensive chromosomal rearrangements. ISME J. 11: 11891204.

Hastie AC (1973) Hybridization of Verticillium albo-atrum and Verticillium dahliae. Trans. Br. Mycol. Soc. 60: 511-523.

Hastie AC (1989) The analysis of Verticillium strain relationship. In Vascular wilt diseases of plants. Tjamos EC \& Beckman CH (eds). Berlin, Heidelberg: Springer. 28: 315-323. 
Heale JB \& Karapapa VK (1999) The Verticillium threat to Canada's major oilseed crop: canola. Can. J. Plant Pathol. 21: 1-7.

Heimel K (2015) Unfolded protein response in filamentous fungi - implications in biotechnology. Appl. Microbiol. Biotechnol. 99: 121-132.

Heimel K, Freitag J, Hampel M \& Ast J (2013) Crosstalk between the unfolded protein response and pathways that regulate pathogenic development in Ustilago maydis. Plant Cell 25: 42624277.

Heimel K, Scherer M, Schuler D \& Kämper J (2010) The Ustilago maydis Clp1 protein orchestrates pheromone and b-dependent signaling pathways to coordinate the cell cycle and pathogenic development. Plant Cell 22: 2908-2922.

Heitman J \& Kronstad JW, Taylor JW, Casselton LA (eds) (2007) Sex in Fungi. WA, USA: American Society of Microbiology 1: 1-542.

Herskowitz I (1995) MAP kinase pathways in yeast: for mating and more. Cell 80: 187-197.

Herzog B, Popova B, Jakobshagen A, Shahpasandzadeh H \& Braus GH (2013) Mutual cross talk between the regulators Hac1 of the unfolded protein response and Gcn4 of the general amino acid control of Saccharomyces cerevisiae. Eukaryot. Cell 12: 1142-1154.

Hetz C (2012) The unfolded protein response: controlling cell fate decisions under ER stress and beyond. Nat. Rev. Mol. Cell Biol. 13: 89-102.

Hicks J, Lockington RA, Strauss J, Dieringer D, Kubicek CP, Kelly J \& Keller N (2001) RcoA has pleiotropic effects on Aspergillus nidulans cellular development. Mol. Microbiol. 39: 14821493.

Hollensteiner J, Wemheuer F, Harting R \& Kolarzyk AM (2017) Bacillus thuringiensis and Bacillus weihenstephanensis inhibit the growth of phytopathogenic Verticillium species. Front. Microbiol. 7: 1-19.

Hooks KB \& Griffiths-Jones S (2011) Conserved RNA structures in the non-canonical Hac1/Xbp1 intron. RNA Biol. 8: 552-556.

Horowitz Brown S, Scott JB, Bhaheetharan J, Sharpee WC, Milde L, Wilson RA \& Keller NP (2009) Oxygenase coordination is required for morphological transition and the host-fungus interaction of Aspergillus flavus. Mol. Plant-Microbe Interact. 22: 882-894.

Horowitz Brown S, Zarnowski R, Sharpee WC \& Keller NP (2008) Morphological transitions governed by density dependence and lipoxygenase activity in Aspergillus flavus. Appl. Environ. Microbiol. 74: 5674-5685.

Hurst HC (1995) Transcription factors 1: bZIP proteins. Protein Profile 2: 101-168.

Husain A, Ahmad A \& Agrawal PK (1993) (-)-Jasmonic acid, a phytotoxic substance from Botryodiplodia theobromae: characterization by NMR spectroscopic methods. J. Nat. Prod. 56: 2008-2011.

Inderbitzin P, Bostock RM, Davis RM, Usami T, Platt HW \& Subbarao KV (2011a) Phylogenetics and taxonomy of the fungal vascular wilt pathogen Verticillium, with the descriptions of five new species. PLoS One 6: e28341.

Inderbitzin P, Davis RM, Bostock RM \& Subbarao KV (2011b) The ascomycete Verticillium longisporum is a hybrid and a plant pathogen with an expanded host range. PLoS One 6: e18260.

Inderbitzin P \& Subbarao KV (2014) Verticillium systematics and evolution: how confusion impedes Verticillium wilt management and how to resolve it. Phytopathology 104: 564-574.

Ingram R (1968) Verticillium dahliae var. Iongisporum, a stable diploid. Trans. Br. Mycol. Soc. 51: 339-341.

Inouye C, Dhillon N \& Thorner J (1997) Ste5 RING-H2 domain: role in Ste4-promoted oligomerization for yeast pheromone signaling. Science 278: 103-106.

Jabnoun-Khiareddine H, Daami-Remadi M, Hibar K, Robb J \& El Mahjoub M (2006) effect of temperature on Verticillium wilts of tomato in Tunisia. Plant Pathol. J. 5: 1-6. 
Jain BP (2019) Genome wide analysis of WD40 proteins in Saccharomyces cerevisiae and their orthologs in Candida albicans. Protein J. 38: 58-75.

Jamet-Vierny C, Debuchy R, Prigent M \& Silar P (2007) IDC1, a Pezizomycotina-specific gene that belongs to the PaMpk1 MAP kinase transduction cascade of the filamentous fungus Podospora anserina. Fungal Genet. Biol. 44: 1219-1230.

Jenczmionka NJ \& Schäfer W (2005) The Gpmk1 MAP kinase of Fusarium graminearum regulates the induction of specific secreted enzymes. Curr. Genet. 47: 29-36.

Jiang C, Zhang X, Liu H \& Xu J-R (2018a) Mitogen-activated protein kinase signaling in plant pathogenic fungi. PLoS Pathog. 14: e1006875.

Jiang $\mathrm{H}$, Lin L, Tang W, Chen X, Zheng Q, Huang J, Yang T, Su L, Dong Y, Wang B \& Wang Z (2018b) Putative interaction proteins of the ubiquitin ligase Hrd1 in Magnaporthe oryzae. Evol. Bioinform. Online 14: 1176934318810990.

Jin L, Chen D, Liao S, Zhang Y, Yu F, Wan P, Yu D, Wu Q \& Zhang Y (2019) Transcriptome analysis reveals downregulation of virulence-associated genes expression in a low virulence Verticillium dahliae strain. Arch. Microbiol. (ahead of print) doi:10.1007/s00203-019-01663-7.

Joazeiro CA \& Weissman AM (2000) RING finger proteins: mediators of ubiquitin ligase activity. Cell 102: 549-552.

Jones P, Binns D, Chang HY, Fraser M, Li W, McAnulla C, McWilliam H, Maslen J, Mitchell A, Nuka G, Pesseat S, Quinn AF, Sangrador-Vegas A, Scheremetjew M, Yong S-Y, Lopez R \& Hunter S (2014) InterProScan 5: genome-scale protein function classification. Bioinformatics 30: $1236-1240$.

Jonikas MC, Collins SR, Denic V, Oh E, Quan EM, Schmid V, Weibezahn J, Schwappach B, Walter P, Weissman JS \& Schuldiner M (2009) Comprehensive characterization of genes required for protein folding in the endoplasmic reticulum. Science 323: 1693-1697.

Jonkers W, Fischer MS, Do HP, Starr TL \& Glass NL (2016) Chemotropism and cell fusion in Neurospora crassa relies on the formation of distinct protein complexes by HAM- 5 and a novel protein HAM-14. Genetics 203: 334-391.

Jonkers W, Leeder AC, Ansong C, Wang Y, Yang F, Starr TL, Camp DG, Smith RD \& Glass NL (2014) HAM-5 functions as a MAP kinase scaffold during cell fusion in Neurospora crassa. PLoS Genet. 10: e1004783.

Joubert A, Simoneau P, Campion C, Poupard P, François JM, Georgeault S, Sellier E \& Guillemette T (2011) Impact of the unfolded protein response on the pathogenicity of the necrotrophic fungus Alternaria brassicicola. Mol. Microbiol. 79: 1305-1324.

Kämper J, Kahmann R, Bölker M, Ma L-J, Brefort T, Saville BJ, Banuett F, Kronstad JW, Gold SE, Müller O, Perlin MH, Wösten HAB, de Vries R, Ruiz-Herrera J, Reynaga-Peña CG, Snetselaar K, McCann M, Pérez-Martín J, Feldbrügge M, Basse CW, et al. (2006) Insights from the genome of the biotrophic fungal plant pathogen Ustilago maydis. Nature 444: 97101.

Karapapa VK, Bainbridge BW \& Heale JB (1997) Morphological and molecular characterization of Verticillium longisporum comb. nov., pathogenic to oilseed rape. Mycol. Res. 101: 12811294.

Kaufman RJ (1999) Stress signaling from the lumen of the endoplasmic reticulum: coordination of gene transcriptional and translational controls. Genes Dev. 13: 1211-1233.

Kersey PJ, Allen JE, Allot A, Barba M, Boddu S, Bolt BJ, Carvalho-Silva D, Christensen M, Davis P, Grabmueller C, Kumar N, Liu Z, Maurel T, Moore B, McDowall MD, Maheswari U, Naamati G, Newman V, Ong CK, Paulini M, et al. (2018) Ensembl Genomes 2018: an integrated omics infrastructure for non-vertebrate species. Nucleic Acids Res. 46: D802-D808.

Klosterman SJ, Atallah ZK, Vallad GE \& Subbarao KV (2009) Diversity, pathogenicity, and management of Verticillium species. Annu. Rev. Phytopathol. 47: 39-62.

Klosterman SJ, Subbarao KV, Kang S, Veronese P, Gold SE, Thomma BPHJ, Chen Z, Henrissat B, Lee YH, Park J, Garcia-Pedrajas MD, Barbara DJ, Anchieta A, de Jonge R, Santhanam P, Maruthachalam K, Atallah Z, Amyotte SG, Paz Z, Inderbitzin P, et al. (2011) Comparative 
genomics yields insights into niche adaptation of plant vascular wilt pathogens. PLoS Pathog. 7: e1002137.

Kohno K (2010) Stress-sensing mechanisms in the unfolded protein response: similarities and differences between yeast and mammals. J. Biochem. 147: 27-33.

Kohno K, Normington K, Sambrook J, Gething MJ \& Mori K (1993) The promoter region of the yeast KAR2 (BiP) gene contains a regulatory domain that responds to the presence of unfolded proteins in the endoplasmic reticulum. Mol. Cell. Biol. 13: 877-890.

Koike ST, Subbarao KV, Davis RM, Gordon TR \& Hubbard J (1994) Verticillium wilt of cauliflower in California. Plant Dis. 78: 1116-1121.

Kolar M, Punt PJ, van den Hondel CAMJJ \& Schwab H (1988) Transformation of Penicillium chrysogenum using dominant selection markers and expression of an Escherichia coli lacZ fusion gene. Gene 62: 127-134.

Kombrink A, Rovenich H, Shi-Kunne X, Rojas-Padilla E, van den Berg GCM, Domazakis E, de Jonge R, Valkenburg DJ, Sánchez-Vallet A, Seidl MF \& Thomma BPHJ (2017) Verticillium dahliae LysM effectors differentially contribute to virulence on plant hosts. Mol. Plant Pathol. 18: 596-608.

Kosugi S, Hasebe M, Tomita M \& Yanagawa H (2009) Systematic identification of cell cycledependent yeast nucleocytoplasmic shuttling proteins by prediction of composite motifs. Proc. Natl. Acad. Sci. 106: 10171-10176.

Kothe E (1996) Tetrapolar fungal mating types: sexes by the thousands. FEMS Microbiol. Rev. 18: $65-87$.

Kozutsumi Y, Segal M, Normington K, Gething M-J \& Sambrook J (1988) The presence of malfolded proteins in the endoplasmic reticulum signals the induction of glucose-regulated proteins. Nature 332: 462-464.

Kranz JE, Satterberg B \& Elion EA (1994) The MAP kinase Fus3 associates with and phosphorylates the upstream signaling component Ste5. Genes Dev. 8: 313-327.

Krishnan K \& Askew DS (2014) The fungal UPR: a regulatory hub for virulence traits in the mold pathogen Aspergillus fumigatus. Virulence 5: 334-340.

Lazo GR, Stein PA \& Ludwig RA (1991) A DNA transformation-competent Arabidopsis genomic library in Agrobacterium. Biotechnology 9: 963-967.

Lev S \& Horwitz BA (2003) A mitogen-activated protein kinase pathway modulates the expression of two cellulase genes in Cochliobolus heterostrophus during plant infection. Society 15: 835844.

Lev S, Sharon A, Hadar R, Ma H \& Horwitz BA (1999) A mitogen-activated protein kinase of the corn leaf pathogen Cochliobolus heterostrophus is involved in conidiation, appressorium formation, and pathogenicity: diverse roles for mitogen-activated protein kinase homologs in foliar pathogens. Proc. Natl. Acad. Sci. U.S.A. 96: 13542-13547.

Li C, Wen A, Shen B, Lu J, Huang Y \& Chang Y (2011) FastCloning: a highly simplified, purification-free, sequence- and ligation-independent PCR cloning method. BMC Biotechnol 11: 92.

Li D, Bobrowicz P, Wilkinson HH \& Ebbole DJ (2005) A mitogen-activated protein kinase pathway essential for mating and contributing to vegetative growth in Neurospora crassa. Genetics 170: $1091-1104$.

Li G, Zhou X \& Xu J-R (2012) Genetic control of infection-related development in Magnaporthe oryzae. Curr. Opin. Microbiol. 15: 678-684.

Li J (2019) Identification of host-specific effectors mediating pathogenicity of the vascular wilt pathogen Verticillium dahliae. Dissertation at the Wageningen University.

Liñeiro E, Chiva C, Cantoral JM, Sabidó E \& Fernández-Acero FJ (2016) Modifications of fungal membrane proteins profile under pathogenicity induction: a proteomic analysis of Botrytis cinerea membranome. Proteomics 16: 2363-2376. 
Liu T, Song T, Zhang X, Yuan H, Su L, Li W, Xu J \& Liu S (2014) Unconventionally secreted effectors of two filamentous pathogens target plant salicylate biosynthesis. Nat. Commun. 5: 4686.

Liu XH, Gao HM, Xu F, Lu JP, Devenish RJ \& Lin FC (2012) Autophagy vitalizes the pathogenicity of pathogenic fungi. Autophagy 8: 1415-1425.

Livak KJ \& Schmittgen TD (2001) Analysis of relative gene expression data using real-time quantitative PCR and the $2^{-\Delta \Delta C_{T}}$ Method. Methods 25: 402-408.

López-Ráez JA, Verhage A, Fernández I, García JM, Azcón-Aguilar C, Flors V \& Pozo MJ (2010) Hormonal and transcriptional profiles highlight common and differential host responses to arbuscular mycorrhizal fungi and the regulation of the oxylipin pathway. J. Exp. Bot. 61: 2589-2601.

Lo Presti L, Lanver D, Schweizer G, Tanaka S, Liang L, Tollot M, Zuccaro A, Reissmann S \& Kahmann R (2015) Fungal effectors and plant susceptibility. Annu. Rev. Plant Biol. 66: 513545.

Los DA \& Murata N (1998) Structure and expression of fatty acid desaturases. Biochim. Biophys. Acta. 1394: 3-15.

Luo X, Xie C \& Dong J (2014) Interactions between Verticillium dahliae and its host: vegetative growth, pathogenicity, plant immunity. Appl. Microbiol. Biotechnol. 98: 6921-6932.

Ma L-J, van der Does HC, Borkovich KA, Coleman JJ, Daboussi M-J, Di Pietro A, Dufresne M, Freitag M, Grabherr F, Henrissat B, Houterman PM, Kang S, Shim W-B, Woloshuk C, Xie X, Xu J-R, Antoniw J, Baker SE, Bluhm BH, Breakspear A, et al. (2010) Comparative genomics reveals mobile pathogenicity chromosomes in Fusarium. Nature 464: 367-373.

Madhani HD \& Fink GR (1998) The riddle of MAP kinase signaling specificity. Trends Genet. 14: 151-155.

Malavazi I, Goldmanm GH \& Brown NA (2014) The importance of connections between the cell wall integrity pathway and the unfolded protein response in filamentous fungi. Brief. Funct. Genomics 13: 456-470.

Malcolm GM, Kuldau GA, Gugino BK \& del Mar Jiménez-Gasco M (2013) Hidden host plant associations of soilborne fungal pathogens: an ecological perspective. Phytopathology 103: 538-544.

Mansilla MC, Cybulski LE, Albanesi D \& de Mendoza D (2004) Control of membrane lipid fluidity by molecular thermosensors. J. Bacteriol. 186: 6681-6688.

Marcus S, Polverino A, Barr M \& Wigler M (1994) Complexes between STE5 and components of the pheromone-responsive mitogen-activated protein kinase module. Proc. Natl. Acad. Sci. U.S.A. 91: 7762-7766.

Martin CE, Oh C-S \& Jiang Y (2007) Regulation of long chain unsaturated fatty acid synthesis in yeast. Biochim. Biophys. Acta. 1771: 271-285.

Mazur P, El-zayat AE, Champe SP (1991) Structure and synthesis of sporogenic psi factors from Aspergillus nidulans. J. Chem. Soc. Chem. Commun. 20: 1486-1487.

Mehrabi R, Bahkali AH, Abd-Elsalam KA, Moslem M, Ben M'Barek S, Gohari AM, Jashni MK, Stergiopoulos I, Kema GHJ \& de Wit PJ (2011) Horizontal gene and chromosome transfer in plant pathogenic fungi affecting host range. FEMS Microbiol. Rev. 35: 542-554.

Menardo F, Praz CR, Wyder S, Ben-David R, Bourras S, Matsumae H, McNally KE, Parlange F, Riba A, Roffler S, Schaefer LK, Shimizu KK, Valenti L, Zbinden H, Wicker T \& Keller B (2016) Hybridization of powdery mildew strains gives rise to pathogens on novel agricultural crop species. Nat. Genet. 48: 201-205.

Metzenberg RL \& Glass NL (1990) Mating type and mating strategies in Neurospora. BioEssays 12: 53-59.

Mey G, Oeser B, Lebrun MH \& Tudzynski P (2002) The biotrophic, non-appressorium-forming grass pathogen Claviceps purpurea needs a Fus3/Pmk1 homologous mitogen-activated protein kinase for colonization of rye ovarian tissue. Mol. Plant. Microbe. Interact. 15: 303312. 
Miersch O, Bohlmann H \& Wasternack C (1999) Jasmonates and related compounds from Fusarium oxysporum. Phytochemistry 50: 517-523.

Milgroom MG, del Mar Jiménez-Gasco M, Olivares García C, Drott MT \& Jiménez-Díaz RM (2014) Recombination between clonal lineages of the asexual fungus Verticillium dahliae detected by genotyping by sequencing. PLoS One 9: e106740.

Miura N \& Ueda M (2018) Evaluation of unconventional protein secretion by Saccharomyces cerevisiae and other fungi. Cells 7: E128.

Miyazaki T, Nakayama H, Nagayoshi Y, Kakeya H \& Kohno S (2013) Dissection of Ire1 functions reveals stress response mechanisms uniquely evolved in Candida glabrata. PLoS Pathog. 9: e1003160.

Money NP (2016) Fungal Cell Biology and Development. In The Fungi. Watkinson SC Boddy L \& Money NP (eds). New York, NY, USA: Academic Press 3: 37-66.

Montenegro-Montero A, Goity A \& Larrondo LF (2015) The bZIP transcription factor HAC-1 is involved in the unfolded protein response and is necessary for growth on cellulose in Neurospora crassa. PLoS One 10: e0131415.

Mori K, Kawahara T, Yoshida H, Yanagi H \& Yura T (1996) Signalling from endoplasmic reticulum to nucleus: transcription factor with a basic-leucine zipper motif is required for the unfolded protein-response pathway. Genes to Cells 1: 803-817.

Mori K, Ma W, Gething MJ \& Sambrook J (1993) A transmembrane protein with a cdc2+/CDC28related kinase activity is required for signaling from the ER to the nucleus. Cell 74: 743-756.

Mori K, Ogawa N, Kawahara T, Yanagi H \& Yura T (1998) Palindrome with spacer of one nucleotide is characteristic of the cis-acting unfolded protein response element in Saccharomyces cerevisiae. J. Biol. Chem. 273: 9912-9920.

Mösch HU, Kübler E, Krappmann S, Fink GR \& Braus GH (1999) Crosstalk between the Ras2pcontrolled mitogen-activated protein kinase and CAMP pathways during invasive growth of Saccharomyces cerevisiae. Mol. Biol. Cell 10: 1325-1335.

Mulder HJ \& Nikolaev I (2009) HacA-dependent transcriptional switch releases hacA mRNA from a translational block upon endoplasmic reticulum stress. Eukaryot. Cell 8: 665-675.

Mulder HJ, Saloheimo M, Penttilä M \& Madrid SM (2004) The transcription factor HACA mediates the unfolded protein response in Aspergillus niger, and up-regulates its own transcription. Mol. Genet. Genomics 271: 130-140.

Mullins ED, Chen X, Romaine P, Raina R, Geiser DM \& Kang S (2001) Agrobacterium-mediated transformation of Fusarium oxysporum: an efficient tool for insertional mutagenesis and gene transfer. 91: 173-180.

Munn AL, Heese-Peck A, Stevenson BJ, Pichler H \& Riezman H (1999) Specific sterols required for the internalization step of endocytosis in yeast. Mol. Biol. Cell 10: 3943-3957.

Murashige T \& Skoog F (1962) A revised medium for rapid growth and bioassays with tobacco tissue cultures. Plant Physiol. 15: 473-497.

Nakamura N (2011) The role of the transmembrane RING finger proteins in cellular and organelle function. Membranes 1: 354-393.

Nguyen VC, Nakamura Y \& Kanehara K (2019) Membrane lipid polyunsaturation mediated by fatty acid desaturase 2 (FAD 2) is involved in endoplasmic reticulum stress tolerance in Arabidopsis thaliana. Plant J. (ahead of print) doi:10.1111/tpj.14338.

Nikawa J, Akiyoshi M, Hirata S \& Fukuda T (1996) Saccharomyces cerevisiae IRE2/HAC1 is involved in IRE1-mediated KAR2 expression. Nucleic Acids Res. 24: 4222-4226.

Novakazi F, Inderbitzin P, Sandoya G, Hayes RJ, von Tiedemann A \& Subbarao KV (2015) The three lineages of the diploid hybrid Verticillium longisporum differ in virulence and pathogenicity. Phytopathology 105: 662-673.

O'Connell RJ, Thon MR, Hacquard S, Amyotte SG, Kleemann J, Torres MF, Damm U, Buiate EA, Epstein L, Alkan N, Altmüller J, Alvarado-Balderrama L, Bauser CA, Becker C, Birren BW, Chen Z, Choi J, Crouch JA, Duvick JP, Farman MA, et al. (2012) Lifestyle transitions in plant 
pathogenic Colletotrichum fungi deciphered by genome and transcriptome analyses. Nat. Genet. 44: 1060-1065.

Oberhofer M \& Leuchtmann A (2012) Genetic diversity in epichloid endophytes of Hordelymus europaeus suggests repeated host jumps and interspecific hybridizations. Mol. Ecol. 21: 2713-2726.

Okamura K, Kimata Y, Higashio H, Tsuru A \& Kohno K (2000) Dissociation of Kar2p/BiP from an ER sensory molecule, Ire1p, triggers the unfolded protein response in yeast. Biochem. Biophys. Res. Commun. 279: 445-450.

Ortoneda M, Guarro J, Madrid MP, Caracuel Z, Roncero MIG, Mayayo E \& Di Pietro A (2004) Fusarium oxysporum as a multihost model for the genetic dissection of fungal virulence in plants and mammals. Infect. Immun. 72: 1760-1766.

Park G, Xue C, Zhao X, Kim Y, Orbach M \& Xu J-R (2006) Multiple upstream signals converge on the adaptor protein Mst50 in Magnaporthe grisea. Plant Cell online 18: 2822-2835.

Park G, Xue C, Zheng L, Lam S \& Xu J-R (2002) MST12 regulates infectious growth but not appressorium formation in the rice blast fungus Magnaporthe grisea. Mol. Plant-Microbe Interact. 15: 183-192.

Passorn S, Laoteng K, Rachadawong S, Tanticharoen M \& Cheevadhanarak S (1999) Heterologous expression of Mucor rouxii $\Delta^{12}$-desaturase gene in Saccharomyces cerevisiae. Biochem. Biophys. Res. Commun. 263: 47-51.

Patkar RN, Benke PI, Qu Z, Chen YYC, Yang F, Swarup S \& Naqvi NI (2015) A fungal monooxygenase-derived jasmonate attenuates host innate immunity. Nat. Chem. Biol. 11: 733-740.

Patkar RN \& Naqvi NI (2017) Fungal manipulation of hormone-regulated plant defense. PLoS Pathog. 13: e1006334.

Patterson JC, Klimenko ES \& Thorner J (2010) Single-cell analysis reveals that insulation maintains signaling specificity between two yeast MAPK pathways with common components. Sci. Signal. 3: ra75.

Pegg GF (1984) The impact of Verticillium diseases in agriculture. Phytopathol. Mediterr. 23: 176192.

Pegg GF \& Brady BL (2002) Verticillium wilts. Pegg GF \& Brady BL (eds) Wallingford, UK: CABI Publishing 1: 1-552.

Perez-Nadales E \& Di Pietro A (2015) The transmembrane protein Sho1 cooperates with the mucin Msb2 to regulate invasive growth and plant infection in Fusarium oxysporum. Mol. Plant Pathol. 16: 593-603.

Di Pietro A, García-Maceira FI, Méglecz E \& Roncero MIG (2001) A MAP kinase of the vascular wilt fungus Fusarium oxysporum is essential for root penetration and pathogenesis. Mol. Microbiol. 39: 1140-1152.

Pinter N, Hach CA, Hampel M, Rekhter D, Zienkiewicz K, Feussner I, Poehlein A, Daniel R, Finkernagel F \& Heimel K (2019) Signal peptide peptidase activity connects the unfolded protein response to plant defense suppression by Ustilago maydis. PLoS Pathog. 15: e1007734.

Piper RC \& Luzio JP (2007) Ubiquitin-dependent sorting of integral membrane proteins for degradation in lysosomes. Curr. Opin. Cell Biol. 19: 459-465.

Plett JM, Daguerre Y, Wittulsky S, Vayssieres A, Deveau A, Melton SJ, Kohler A, Morrell-Falvey JL, Brun A, Veneault-Fourrey C \& Martin F (2014) Effector MiSSP7 of the mutualistic fungus Laccaria bicolor stabilizes the Populus JAZ6 protein and represses jasmonic acid (JA) responsive genes. Proc. Natl. Acad. Sci. 111: 8299-8304.

Plissonneau C, Hartmann FE \& Croll D (2018) Pangenome analyses of the wheat pathogen Zymoseptoria tritici reveal the structural basis of a highly plastic eukaryotic genome. BMC Biol. 16: 5 .

Pöggeler S \& Kück U (2004) A WD40 repeat protein regulates fungal cell differentiation and can be replaced functionally by the mammalian homologue striatin. Eukaryot. Cell 3: 232-240. 
Printen JA \& Sprague GF (1994) Protein-protein interactions in the yeast pheromone response pathway: Ste5p interacts with all members of the MAP kinase cascade. Genetics 138: 609619.

Pryciak PM \& Huntress FA (1998) Membrane recruitment of the kinase cascade scaffold protein Ste5 by the Gbetagamma complex underlies activation of the yeast pheromone response pathway. Genes Dev. 12: 2684-2697.

Punt PJ, Dingemanse MA, Jacobs-Meijsing BJM, Pouwels PH \& van den Hondel CAMJJ (1988) Isolation and characterization of the glyceraldehyde-3-phosphate dehydrogenase gene of Aspergillus nidulans. Gene 69: 49-57.

Qi X, Zhou S, Shang X \& Wang X (2016) VdSho1 regulates growth, oxidant adaptation and virulence in Verticillium dahliae. J. Phytopathol. 164: 1064-1074.

Qin L, Wu VW \& Glass NL (2017) Deciphering the regulatory network between the SREBP pathway and protein secretion in Neurospora crassa. MBio 8: e00233-17.

Raffaele S \& Kamoun S (2012) Genome evolution in filamentous plant pathogens: why bigger can be better. Nat. Rev. Microbiol. 10: 417-430.

Ralhan A, Schöttle S, Thurow C, Iven T, Feussner I, Polle A \& Gatz C (2012) The vascular pathogen Verticillium longisporum requires a jasmonic acid-independent COl1 function in roots to elicit disease symptoms in Arabidopsis shoots. Plant Physiol. 159: 1192-1203.

Rambo GW \& Bean GA (1974) Sterols and fatty acids of aflatoxin and non-aflatoxin producing isolates of Aspergillus. Phytochemistry 13: 195-198.

Rana A, Sahgal M \& Johri BN (2017) Fusarium oxysporum: genomics, diversity and plant-host interaction. In Developments in fungal biology and applied mycology. Satyanarayana T, Deshmukh SK \& Johri BN (eds). Berlin, Heidelberg: Springer 1:159-199.

Rasband W (1997) Rasband. ImageJ, U. S. Natl. Institutes Heal. Bethesda, Maryland, USA. Available at: http://imagej.nih.gov/ij.

Rauyaree P, Ospina-Giraldo MD, Kang S, Bhat RG, Subbarao KV, Grant SJ \& Dobinson KF (2005) Mutations in VMK1, a mitogen-activated protein kinase gene, affect microsclerotia formation and pathogenicity in Verticillium dahliae. Curr. Genet. 48: 109-116.

Resende ML V., Flood J \& Cooper RM (1994) Host specialization of Verticillium dahliae, with emphasis on isolates from cocoa (Theobroma cacao). Plant Pathol. 43: 104-111.

Reusche M, Truskina J, Thole K, Nagel L, Rindfleisch S, Tran VT, Braus-Stromeyer SA, Braus GH, Teichmann T \& Lipka V (2014) Infections with the vascular pathogens Verticillium longisporum and Verticillium dahliae induce distinct disease symptoms and differentially affect drought stress tolerance of Arabidopsis thaliana. Environ. Exp. Bot. 108: 23-37.

Reverberi M, Punelli F, Scarpari M, Camera E, Zjalic S, Ricelli A, Fanelli C \& Fabbri AA (2010) Lipoperoxidation affects ochratoxin A biosynthesis in Aspergillus ochraceus and its interaction with wheat seeds. Appl. Microbiol. Biotechnol. 85: 1935-1946.

Richie DL, Feng X, Hartl L, Aimanianda V, Krishnan K, Powers-Fletcher M V, Watson DS, Galande AK, White SM, Willett T, Latgé J-P, Rhodes JC \& Askew DS (2011) The virulence of the opportunistic fungal pathogen Aspergillus fumigatus requires cooperation between the endoplasmic reticulum-associated degradation pathway (ERAD) and the unfolded protein response (UPR). Virulence 2: 12-21.

Richie DL, Hartl L, Aimanianda V, Winters MS, Fuller KK, Miley MD, White S, McCarthy JW, Latgé J-P, Feldmesser M, Rhodes JC \& Askew DS (2009) A role for the unfolded protein response (UPR) in virulence and antifungal susceptibility in Aspergillus fumigatus. PLoS Pathog. 5: e1000258.

Riemann M, Haga K, Shimizu T, Okada K, Ando S, Mochizuki S, Nishizawa Y, Yamanouchi U, Nick P, Yano M, Minami E, Takano M, Yamane H \& Lino M (2013) Identification of rice Allene Oxide Cyclase mutants and the function of jasmonate for defence against Magnaporthe oryzae. Plant J. 74: 226-238.

Rispail N \& Di Pietro A (2009) Fusarium oxysporum Ste12 controls invasive growth and virulence downstream of the Fmk1 MAPK cascade. Mol. Plant-Microbe Interact. 22: 830-839. 
Rispail N, Soanes DM, Ant C, Czajkowski R, Grünler A, Huguet R, Perez-Nadales E, Poli A, Sartorel E, Valiante V, Yang M, Beffa R, Brakhage AA, Gow NAR, Kahmann R, Lebrun M-H, Lenasi H, Perez-Martin J, Talbot NJ, Wendland J, et al. (2009) Comparative genomics of MAP kinase and calcium-calcineurin signalling components in plant and human pathogenic fungi. Fungal Genet. Biol. 46: 287-298.

Ron D \& Walter $P$ (2007) Signal integration in the endoplasmic reticulum unfolded protein response. Nat. Rev. Mol. Cell Biol. 8: 519-529.

Rouxel T, Grandaubert J, Hane JK, Hoede C, van de Wouw AP, Couloux A, Dominguez V, Anthouard V, Bally P, Bourras S, Cozijnsen AJ, Ciuffetti LM, Degrave A, Dilmaghani A, Duret L, Fudal I, Goodwin SB, Gout L, Glaser N, Linglin J, et al. (2011) Effector diversification within compartments of the Leptosphaeria maculans genome affected by repeat-induced point mutations. Nat. Commun. 2: 202.

Rüegsegger U, Leber JH \& Walter P (2001) Block of HAC1 mRNA translation by long-range base pairing is released by cytoplasmic splicing upon induction of the unfolded protein response. Cell 107: 103-114.

Rui O \& Hahn M (2007) The Slt2-type MAP kinase Bmp3 of Botrytis cinerea is required for normal saprotrophic growth, conidiation, plant surface sensing and host tissue colonization. Mol. Plant Pathol. 8: 173-184.

Ruiz-Roldán MC, Maier FJ \& Schäfer W (2001) PTK1, a mitogen-activated-protein kinase gene, is required for conidiation, appressorium formation, and pathogenicity of Pyrenophora teres on barley. Mol. Plant-Microbe Interact. 14: 116-125.

Sabbagh Jr. W, Flatauer LJ, Bardwell AJ \& Bardwell L (2001) Specificity of MAP kinase signaling in yeast differentiation involves transient versus sustained MAPK activation. Mol. Cell 8: 683691.

Sakuradani E, Kobayashi M, Ashikari T \& Shimizu S (1999) Identification of $\Delta 12$-fatty acid desaturase from arachidonic acid-producing Mortierella fungus by heterologous expression in the yeast Saccharomyces cerevisiae and the fungus Aspergillus oryzae. Eur. J. Biochem. 261: 812-820.

Saloheimo M, Valkonen M \& Penttilä M (2003) Activation mechanisms of the HACl-mediated unfolded protein response in filamentous fungi. Mol. Microbiol. 47: 1149-1161.

Samji T, Hong S \& Means RE (2014) The membrane associated RING-CH proteins: a family of E3 ligases with diverse roles through the cell. Int. Sch. Res. Not. 2014: 637295.

Sanders IR (2011) Mycorrhizal symbioses: how to be seen as a good fungus. Curr. Biol. 21: R550-552.

Santhanam P, Esse HP Van, Albert I, Faino L, Nürnberger T \& Thomma BPHJ (2013) Evidence for functional diversification within a fungal NEP1-like protein family. Mol. Plant Pathol. 26: 278-286.

Sarmiento-Villamil JL, Prieto P, Klosterman SJ \& Garcia-Pedrajas, Maria D (2018) Characterization of two homeodomain transcription factors with critical but distinct roles in virulence in the vascular pathogen Verticillium dahliae. Mol. Plant Pathol. 19: 986-1004.

Scala V, Giorni P, Cirlini M, Ludovici M, Visentin I, Cardinale F, Fabbri AA, Fanelli C, Reverberi M, Battilani P, Galaverna G \& Dall'Asta C (2014) LDS1-produced oxylipins are negative regulators of growth, conidiation and fumonisin synthesis in the fungal maize pathogen Fusarium verticillioides. Front. Microbiol. 5: 669.

Schaeffer HJ \& Weber MJ (1999) Mitogen-activated protein kinases: specific messages from ubiquitous messengers. Mol. Cell. Biol. 19: 2435-2444.

Scholz SS, Schmidt-Heck W, Guthke R, Furch ACU, Reichelt M, Gershenzon J \& Oelmüller R (2018) Verticillium dahliae-Arabidopsis interaction causes changes in gene expression profiles and jasmonate levels on different time scales. Front. Microbiol. 9: 217.

Schröder M, Chang JS \& Kaufman RJ (2000) The unfolded protein response represses nitrogenstarvation induced developmental differentiation in yeast. Genes Dev. 14: 2962-2975.

Schwarz DS \& Blower MD (2016) The endoplasmic reticulum: structure, function and response to cellular signaling. Cell. Mol. Life Sci. 73: 79-94. 
Scrimale T, Didone L, de Mesy Bentley KL \& Krysan DJ (2009) The unfolded protein response is induced by the cell wall integrity mitogen-activated protein kinase signaling cascade and is required for cell wall integrity in Saccharomyces cerevisiae. Mol. Biol. Cell 20: 164-175.

Segorbe D, Di Pietro A, Pérez-Nadales E \& Turrà D (2017) Three Fusarium oxysporum mitogenactivated protein kinases (MAPKs) have distinct and complementary roles in stress adaptation and cross-kingdom pathogenicity. Mol. Plant Pathol. 18: 912-924.

Seidl MF \& Thomma BPHJ (2014) Sex or no sex: evolutionary adaptation occurs regardless. BioEssays 36: 335-345.

Selin C, de Kievit TR, Belmonte MF \& Fernando WGD (2016) Elucidating the role of effectors in plant-fungal interactions: progress and challenges. Front. Microbiol. 7: 600.

Shamu CE \& Walter P (1996) Oligomerization and phosphorylation of the Ire1p kinase during intracellular signaling from the endoplasmic reticulum to the nucleus. EMBO J. 15: 30283039.

Sharma R, Mishra B, Runge F \& Thines M (2014) Gene loss rather than gene gain is associated with a host jump from monocots to dicots in the smut fungus Melanopsichium pennsylvanicum. Genome Biol. Evol. 6: 2034-2049.

Shi-kunne X \& Faino L (2018) Evolution within the fungal genus Verticillium is characterized by chromosomal rearrangement and gene loss. Environ. Microbiol. 20: 1362-1373.

Short DPG, Gurung S, Hu X, Inderbitzin P \& Subbarao KV (2014) Maintenance of sex-related genes and the Co-occurrence of both mating types in Verticillium dahliae. PLoS One 9: e112145.

Sidrauski C, Cox JS \& Walter P (1996) tRNA ligase is required for regulated mRNA splicing in the unfolded protein response. Cell 87: 405-413.

Sidrauski C \& Walter P (1997) The transmembrane kinase Ire1p is a site-specific endonuclease that initiates mRNA splicing in the unfolded protein response. Cell 90: 1031-1039.

Siebold M \& von Tiedemann A (2013) Effects of experimental warming on fungal disease progress in oilseed rape. Glob. Chang. Biol. 19: 1736-1747.

Smith BJ (1984) SDS polyacrylamide gel electrophoresis of proteins. In Methods in Molecular Biology. Walker JM (ed). Clifton, NJ, USA: Humana Press 1: 41-55.

Smith G (1949) The effect of adding trace elements to Czapek-Dox medium. Transactions of the British Mycological Society. 32: 280-283.

Soesanto L \& Termorshuizen AJ (2001) Effect of temperature on the formation of microsclerotia of Verticillium dahliae. J. Phytopathol. 149: 685-691.

Soliday CL \& Kolattukudy PE (1977) Biosynthesis of Cutin. Plant Physiol. 59: 1116-1121.

Song Y, Liu L, Wang Y, Valkenburg D-J, Zhang X, Zhu L \& Thomma BPHJ (2018) Transfer of tomato immune receptor Ve1 confers Ave1-dependent Verticillium resistance in tobacco and cotton. Plant Biotechnol. J. 16: 638-648.

Southern EM (1975) Detection of specific sequences among DNA fragments separated by gel electrophoresis. J. Mol. Biol. 98: 503-517.

Stergiopoulos I \& de Wit PJ (2009) Fungal effector proteins. Annu. Rev. Phytopathol. 47: 233263.

Stukenbrock EH, Christiansen FB, Hansen TT, Dutheil JY \& Schierup MH (2012) Fusion of two divergent fungal individuals led to the recent emergence of a unique widespread pathogen species. Proc. Natl. Acad. Sci. U.S.A. 109: 10954-10959.

Su X, Lu G, Rehman L, Li X, Sun L, Guo H \& Cheng H (2018) mCherry-labeled Verticillium dahliae could be utilized to investigate its pathogenicity process in Nicotiana benthamiana. Genes 9: 508.

Su X, Schmitz G, Zhang M, Mackie RI \& Cann IKO (2012) Heterologous gene expression in filamentous fungi. In Advances in Applied Microbiology. Gadd GM \& Sariaslani S (eds). Urbana, IL, USA: Academic Press Inc 81: 1-61. 
Subbarao KV, Kabir Z, Martin FN \& Koike ST (2007) Management of soilborne diseases in strawberry using vegetable rotations. Plant Dis. 91: 964-972.

Sverdlov E \& Azhikina T (2005) Primer Walking. Encycl. Life Sci. doi:10.1038/npg.els.0005382.

Takano Y, Kikuchi T, Kubo Y, Hamer JE, Mise K \& Furusawa I (2000) The Colletotrichum lagenarium MAP kinase gene CMK1 regulates diverse aspects of fungal pathogenesis. Mol. Plant-Microbe Interact. 13: 374-383.

Takemoto D, Kamakura S, Saikia S, Becker Y, Wrenn R, Tanaka A, Sumimoto H \& Scott B (2011) Polarity proteins Bem1 and Cdc24 are components of the filamentous fungal NADPH oxidase complex. Proc. Natl. Acad. Sci. 108: 2861-2866.

Takemoto D, Tanaka A \& Scott B (2006) A p67Phox-like regulator is recruited to control hyphal branching in a fungal-grass mutualistic symbiosis. The Plant Cell Online 18: 2807-2821.

Tamura K, Stecher G, Peterson D, Filipski A \& Kumar S (2013) MEGA6: Molecular Evolutionary Genetics Analysis version 6.0. Mol. Biol. Evol. 30: 2725-2729.

Tanaka A, Christensen MJ, Takemoto D, Park P \& Scott B (2006) Reactive oxygen species play a role in regulating a fungus-perennial ryegrass mutualistic interaction. Plant Cell 18: 10521066.

Tanaka A, Takemoto D, Hyon G-S, Park P \& Scott B (2008) NoxA activation by the small GTPase RacA is required to maintain a mutualistic symbiotic association between Epichloë festucae and perennial ryegrass. Mol. Microbiol. 68: 1165-1178.

Tang W, Ru Y, Hong L, Zhu Q, Zuo R, Guo X, Wang J, Zhang H, Zheng X, Wang P \& Zhang Z (2015) System-wide characterization of bZIP transcription factor proteins involved in infection-related morphogenesis of Magnaporthe oryzae. Environ. Microbiol. 17: 1377-1396.

Tang Y-C \& Amon A (2013) Gene copy-number alterations: a cost-benefit analysis. Cell 152: 394405.

Thaler JS, Owen B \& Higgins VJ (2004) The role of the jasmonate response in plant susceptibility to diverse pathogens with a range of lifestyles. Plant Physiol. 135: 530-538.

Thatcher LF, Manners JM \& Kazan K (2009) Fusarium oxysporum hijacks COl1-mediated jasmonate signaling to promote disease development in Arabidopsis. Plant J. 58: 927-939.

Thines B, Katsir L, Melotto M, Niu Y, Mandaokar A, Liu G, Nomura K, He SY, Howe GA \& Browse $\mathrm{J}$ (2007) JAZ repressor proteins are targets of the SCFCOl1 complex during jasmonate signalling. Nature 448: 661-665.

Thompson JD, Higgins DG \& Gibson TJ (1994) ClustalW: improving the sensitivity of progressive multiple sequence alignment through sequence weighting, position-specific gap penalties and weight matrix choice. Nucleic Acids Res. 22: 4673-4680.

Tian L, Wang Y, Yu J, Xiong D, Zhao H \& Tian C (2016) The Mitogen-activated protein kinase kinase VdPbs2 of Verticillium dahliae regulates microsclerotia formation, stress response, and plant infection. Front. Microbiol. 7: 1532.

Tian L, Xu J, Zhou L \& Guo W (2014) VdMsb regulates virulence and microsclerotia production in the fungal plant pathogen Verticillium dahliae. Gene 550: 238-244.

Timpner C, Braus-Stromeyer SA, Tran VT \& Braus GH (2013) The Cpc1 regulator of the crosspathway control of amino acid biosynthesis is required for pathogenicity of the vascular pathogen Verticillium longisporum. Mol. Plant-Microbe Interact. 26: 1312-1324.

Tran VT, Braus-Stromeyer SA, Kusch H, Reusche M, Kaever A, Kühn A, Valerius O, Landesfeind M, Aßhauer K, Tech M, Hoff K, Pena-Centeno T, Stanke M, Lipka V \& Braus GH (2014) Verticillium transcription activator of adhesion Vta2 suppresses microsclerotia formation and is required for systemic infection of plant roots. New Phytol. 202: 565-581.

Tran VT, Braus-Stromeyer SA, Timpner C \& Braus GH (2013) Molecular diagnosis to discriminate pathogen and apathogen species of the hybrid Verticillium longisporum on the oilseed crop Brassica napus. Appl. Microbiol. Biotechnol. 97: 4467-4483.

Travers KJ, Patil CK, Wodicka L, Lockhart DJ, Weissman JS \& Walter P (2000) Functional and genomic analyses reveal an essential coordination between the unfolded protein response and ER-associated degradation. Cell 101: 249-258. 
Tsitsigiannis DI, Bok J-W, Andes D, Nielsen KF, Frisvad JC \& Keller NP (2005a) Aspergillus cyclooxygenase-like enzymes are associated with prostaglandin production and virulence. Infect. Immun. 73: 4548-4559.

Tsitsigiannis DI \& Keller NP (2006) Oxylipins act as determinants of natural product biosynthesis and seed colonization in Aspergillus nidulans. Mol. Microbiol. 59: 882-892.

Tsitsigiannis DI \& Keller NP (2007) Oxylipins as developmental and host-fungal communication signals. Trends Microbiol. 15: 109-118.

Tsitsigiannis DI, Kowieski TM, Zarnowski R \& Keller NP (2004a) Endogenous lipogenic regulators of spore balance in Aspergillus nidulans. Eukaryot. Cell 3: 1398-1411.

Tsitsigiannis DI, Kowieski TM, Zarnowski R \& Keller NP (2005b) Three putative oxylipin biosynthetic genes integrate sexual and asexual development in Aspergillus nidulans. Microbiology 151: 1809-1821.

Tsitsigiannis DI, Zarnowski R \& Keller NP (2004b) The lipid body protein, PpoA, coordinates sexual and asexual sporulation in Aspergillus nidulans. J. Biol. Chem. 279: 11344-11353.

Turgeon BG \& Yoder OC (2000) Proposed nomenclature for mating type genes of filamentous ascomycetes. Fungal Genet. Biol. 31: 1-5.

Turrà D, Segorbe D \& Di Pietro A (2014) Protein kinases in plant-pathogenic fungi: conserved regulators of infection. Annu. Rev. Phytopathol. 52: 267-288.

Tzima A, Paplomatas EJ, Rauyaree P \& Kang S (2010) Roles of the catalytic subunit of cAMPdependent protein kinase $A$ in virulence and development of the soilborne plant pathogen Verticillium dahliae. Fungal Genet. Biol. 47: 406-415.

Tzima AK, Paplomatas EJ, Tsitsigiannis DI \& Kang S (2012) The G protein b subunit controls virulence and multiple growth- and development-related traits in Verticillium dahliae. Fungal Genet. Biol. 49: 271-283.

Uttaro AD (2006) Biosynthesis of polyunsaturated fatty acids in lower eukaryotes. IUBMB Life 58: 563-571.

Van Drogen F, Stucke VM, Jorritsma G \& Peter M (2001) MAP kinase dynamics in response to pheromones in budding yeast. Nat. Cell Biol. 3: 1051-1059.

Vallad GE \& Subbarao KV (2008) Colonization of resistant and susceptible lettuce cultivars by a green fluorescent protein-tagged isolate of Verticillium dahliae. Phytopathology 98: 871-885.

Velásquez AC, Castroverde CDM \& He SY (2018) Plant-Pathogen warfare under changing climate conditions. Curr. Biol. 28: R619-R634.

Wang G, Zhang D \& Chen S (2014) Effect of earlier unfolded protein response and efficient protein disposal system on cellulase production in Rut C30. World J. Microbiol. Biotechnol. 30: 2587-2595.

Wang Y, Hu X, Fang Y, Anchieta A, Goldman PH, Hernandez G \& Klosterman SJ (2018) Transcription factor $\mathrm{VdCmr1}$ is required for pigment production, protection from UV irradiation, and regulates expression of melanin biosynthetic genes in Verticillium dahliae. Microbiology 164: 685-696.

Wang Y, Tian L, Xiong D, Klosterman SJ, Xiao S \& Tian C (2016) The mitogen-activated protein kinase gene, VdHog1, regulates osmotic stress response, microsclerotia formation and virulence in Verticillium dahliae. Fungal Genet. Biol. 88: 13-23.

Watanabe K, Oura T, Sakai H \& Kajiwara S (2004) Yeast $\Delta 12$ fatty acid desaturase: gene cloning, expression, and function. Biosci. Biotechnol. Biochem. 68: 721-727.

Wei DS, Li MC, Zhang XX, Zhou H \& Xing LJ (2006) A novel $\Delta^{12}$-fatty acid desaturase gene from methylotrophic yeast Pichia pastoris GS115. Acta Biochim. Pol. 53: 753-759.

Welihinda AA \& Kaufman RJ (1996) The unfolded protein response pathway in Saccharomyces cerevisiae. Oligomerization and trans-phosphorylation of Ire1p (Ern1p) are required for kinase activation. J. Biol. Chem. 271: 18181-18187.

Widmann C, Gibson S, Jarpe MB \& Johnson GL (1999) Mitogen-activated protein kinase: conservation of a three-kinase module from yeast to human. Physiol. Rev. 79: 143-180. 
Wilhelm S (1955) Longevity of the Verticillium wilt fungus in the laboratory and field. Phytopath 45: $180-181$.

Williams GC (1975) Sex and evolution. In Monographs in Population Biology. Princeton, NJ, USA: Princeton University Press 8: 170-193.

Wilson RA, Calvo AM, Chang P-K \& Keller NP (2004) Characterization of the Aspergillus parasiticus $\Delta^{12}$-desaturase gene: a role for lipid metabolism in the Aspergillus-seed interaction. Microbiology 150: 2881-2888.

Wimalasena TT, Enjalbert B, Guillemette T, Plumridge A, Budge S, Yin Z, Brown AJP \& Archer DB (2008) Impact of the unfolded protein response upon genome-wide expression patterns, and the role of Hac1 in the polarized growth, of Candida albicans. Fungal Genet. Biol. 45: 1235-1247.

Woodcock DM, Crowther PJ, Doherty J, Jefferson S, DeCruz E, Noyer-Weidner M, Smith SS, Michael MZ \& Graham MW (1989) Quantitative evaluation of Escherichia coli host strains for tolerance to cytosine methylation in plasmid and phage recombinants. Nucleic Acids Res. 17: 3469-3478.

Wu C, Leberer E, Thomas DY \& Whiteway M (1999) Functional characterization of the interaction of Ste50p with Ste11p MAPKKK in Saccharomyces cerevisiae. Mol. Biol. Cell 10: 2425-1440.

Xiong D, Wang Y, Ma J, Klosterman SJ, Xiao S \& Tian C (2014) Deep mRNA sequencing reveals stage-specific transcriptome alterations during microsclerotia development in the smoke tree vascular wilt pathogen, Verticillium dahliae. BMC Genomics 15: 1-19.

Xu C \& Min J (2011) Structure and function of WD40 domain proteins. Protein Cell 2: 202-214.

Xu G, Jansen G, Thomas DY, Hollenberg CP \& Ramezani Rad M (1996) Ste50p sustains mating pheromone-induced signal transduction in the yeast Saccharomyces cerevisiae. Mol. Microbiol. 20: 773-783.

Xu J-R (2000) MAP kinases in fungal pathogens. Fungal Genet. Biol. 31: 137-152.

Xu JR \& Hamer JE (1996) MAP kinase and cAMP signaling regulate infection structure formation and pathogenic growth in the rice blast fungus Magnaporthe grisea. Genes Dev. 10: 26962706.

Yadeta KA \& Thomma BPHJ (2013) The xylem as battleground for plant hosts and vascular wilt pathogens. Front. Plant Sci. 4: 97.

Yi M, Chi M-H, Khang CH, Park S-Y, Kang S, Valent B \& Lee Y-H (2009) The ER chaperone LHS1 is involved in asexual development and rice infection by the blast fungus Magnaporthe oryzae. Plant Cell online 21: 681-695.

Yoshida H, Matsui T, Yamamoto A, Okada T \& Mori $\mathrm{K}$ (2001) XBP1 mRNA is induced by ATF6 and spliced by IRE1 in response to ER stress to produce a highly active transcription factor. Cell 107: 881-891.

Yoshida H, Oku M, Suzuki M \& Mori K (2006) pXBP1U encoded in XBP1 pre-mRNA negatively regulates unfolded protein response activator $\mathrm{pXBP} 1^{\mathrm{s}}$ in mammalian ER stress response. $J$. Cell Biol. 172: 565-575.

Yoshida $\mathrm{H}$, Uemura A \& Mori $\mathrm{K}$ (2009) pXBP1U, a negative regulator of the unfolded protein response activator $\mathrm{pXBP} 1^{\mathrm{s}}$, targets ATF6 but not ATF4 in proteasome-mediated degradation. Cell Struct. Funct. 34: 1-10.

Zeise K \& von Tiedemann A (2002) Host specialization among vegetative compatibility groups of Verticillium dahliae in relation to Verticillium longisporum. J. Phytopathol. 150: 112-119.

Zhang Y, Tada T, Ozono S, Yao W, Tanaka M, Yamaoka S, Kishigami S, Fujita H \& Tokunaga K (2019) Membrane-associated RING-CH (March) 1 and 2 are March family members that inhibit HIV-1 infection. J. Biol. Chem. 294: 3397-3405.

Zhao P, Zhao Y-L, Jin Y, Zhang T \& Guo H-S (2014) Colonization process of Arabidopsis thaliana roots by a green fluorescent protein-tagged isolate of Verticillium dahliae. Protein Cell 5: 9498.

Zhao X, Kim Y, Park G \& Xu J-R (2005) A mitogen-activated protein kinase cascade regulating infection-related morphogenesis in Magnaporthe grisea. Plant Cell 17: 1317-1329. 
Zhao X, Mehrabi R \& Xu JR (2007) Mitogen-activated protein kinase pathways and fungal pathogenesis. Eukaryot. Cell 6: 1701-1714.

Zhao X \& Xu J-R (2007) A highly conserved MAPK-docking site in Mst7 is essential for Pmk1 activation in Magnaporthe grisea. Mol. Microbiol. 63: 881-894.

Zhao Y, Zhou T \& Guo H (2016) Hyphopodium-specific VdNoxB/VdPIs1- dependent ROS-Ca ${ }^{2+}$ signaling is required for plant infection by Verticillium dahliae. PLOS Pathog. 12: e1005793.

Zheng J, Tang C, Deng C \& Wang Y (2019) Involvement of a response regulator VdSsk1 in stress response, melanin biosynthesis and full virulence in Verticillium dahliae. Front. Microbiol. 10: 1-10.

Zheng L, Campbell M, Murphy J, Lam S \& Xu J-R (2000) The BMP1 Gene Is Essential for Pathogenicity in the Gray Mold Fungus Botrytis cinerea. Mol. Plant-Microbe Interact. 13: 724732.

Zhou L, Hu Q, Johansson A \& Dixelius C (2006) Verticillium longisporum and V. dahliae: infection and disease in Brassica napus. Plant Pathol. 55: 137-144.

Zuker M (2003) Mfold web server for nucleic acid folding and hybridization prediction. Nucleic Acids Res. 31: 3406-3415. 


\section{Supplementary Figures}

A

VI43 WT
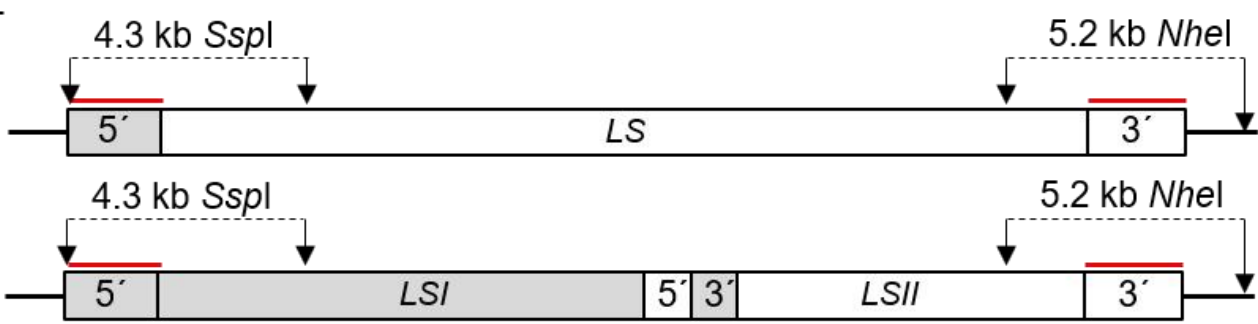

VI43 $\Delta L S I \quad 6.3 \mathrm{~kb} S s p l$

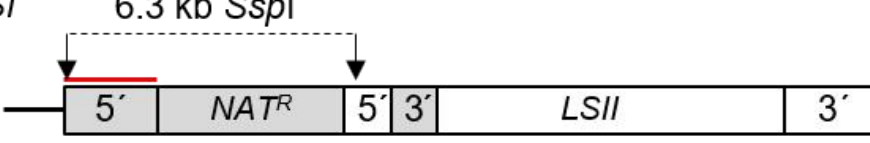

VI43 $\Delta L S I I$

$7.4 \mathrm{~kb}$ Nhel

\begin{tabular}{|l|l|l|l|l|l|}
\hline $5^{\prime}$ & $L S I$ & $5^{\prime}$ & $3^{\prime}$ & $N^{\prime}$ & $3^{\prime}$ \\
\hline
\end{tabular}

VI43 $\Delta L S$

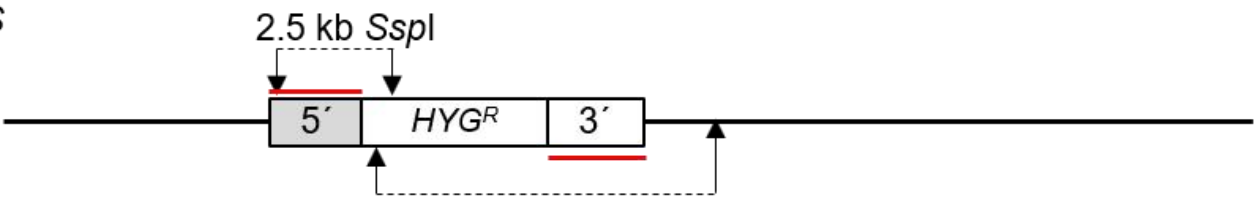

$6.5 \mathrm{~kb}$ Nhel

B
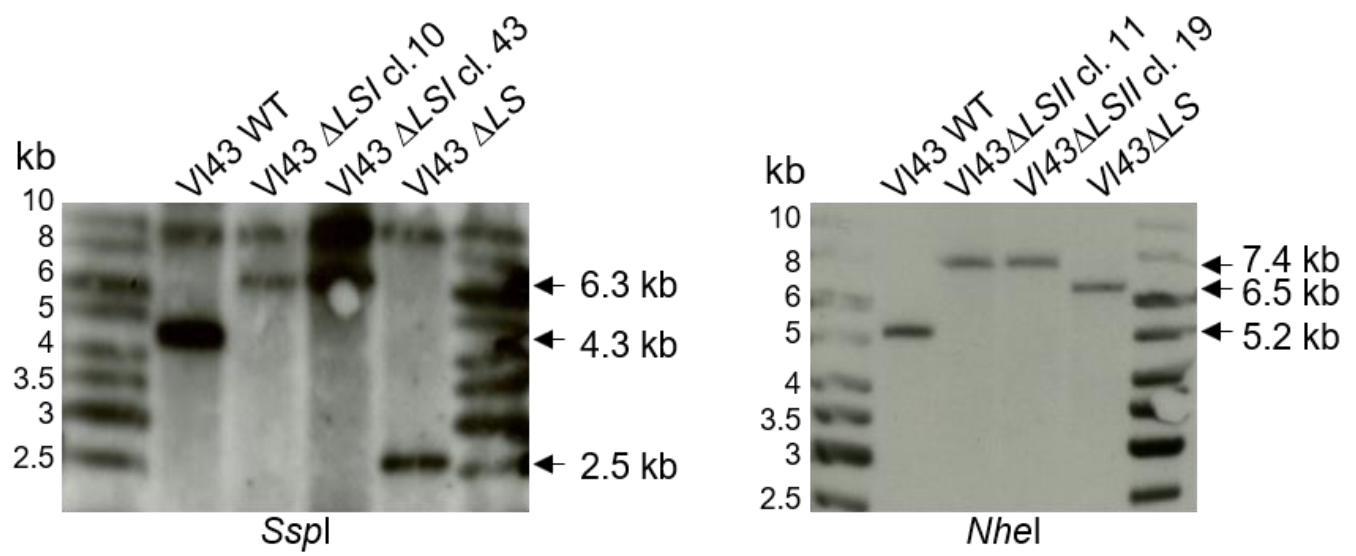

Figure S1: Southern hybridization of V. longisporum VI43 LSI, LSII, and LS deletion

strains. (A) VI43 $\Delta L S I, \Delta L S I I$, and $\Delta L S$ strains were constructed by replacement of genomic regions against nourseothricin or hygromycin resistance marker cassettes under control of a gpdA promoter and a trpC terminator $\left(N A T^{R}\right.$ or $\left.H Y G^{R}\right)$ by homologous recombination of the up- and downstream flanking regions. The VI43LS20kb region was dissected into a $11.5 \mathrm{bp}$ $L S /$ region and a 8.5 bp $L S$ Il region. The $L S I$ deletion strain was used as parental strain of the $L S$ deletion strain. Restriction sites of $S s p l$ and Nhel used for Southern hybridization analysis are indicated by arrows. Expected fragment lengths labeled with the 5'- or 3'flanking regions as probes (indicated in red) are given. (B) Confirmation of VI43 LSI, LSII, and $L S$ deletion strains by Southern hybridization. Left: $2.0 \mathrm{~kb} 5^{\prime}$-flanking region was used as a probe and genomic DNA was cut using the restriction enzyme Sspl. The predicted bands of $4.3 \mathrm{~kb}$ for VI43 wild type, $6.3 \mathrm{~kb}$ for $L S I$ deletion transformants number 10 (VGB201) and 43 (VGB233), and $2.5 \mathrm{~kb}$ for the LS deletion strain (VGB232), and an additional unspecific band at $\sim 8 \mathrm{~kb}$ present in all genotypes were obtained. Right: the $2.0 \mathrm{~kb} 3^{\prime}$-flanking region was used as probe and genomic DNA was cut using the restriction enzyme Nhel. The predicted bands of $5.2 \mathrm{~kb}$ for VI43 wild type, $7.4 \mathrm{~kb}$ for LSII deletion transformants 11 (VGB193) and 19 (VGB214), and $6.5 \mathrm{~kb}$ for the LS deletion strain (VGB232) were obtained. 


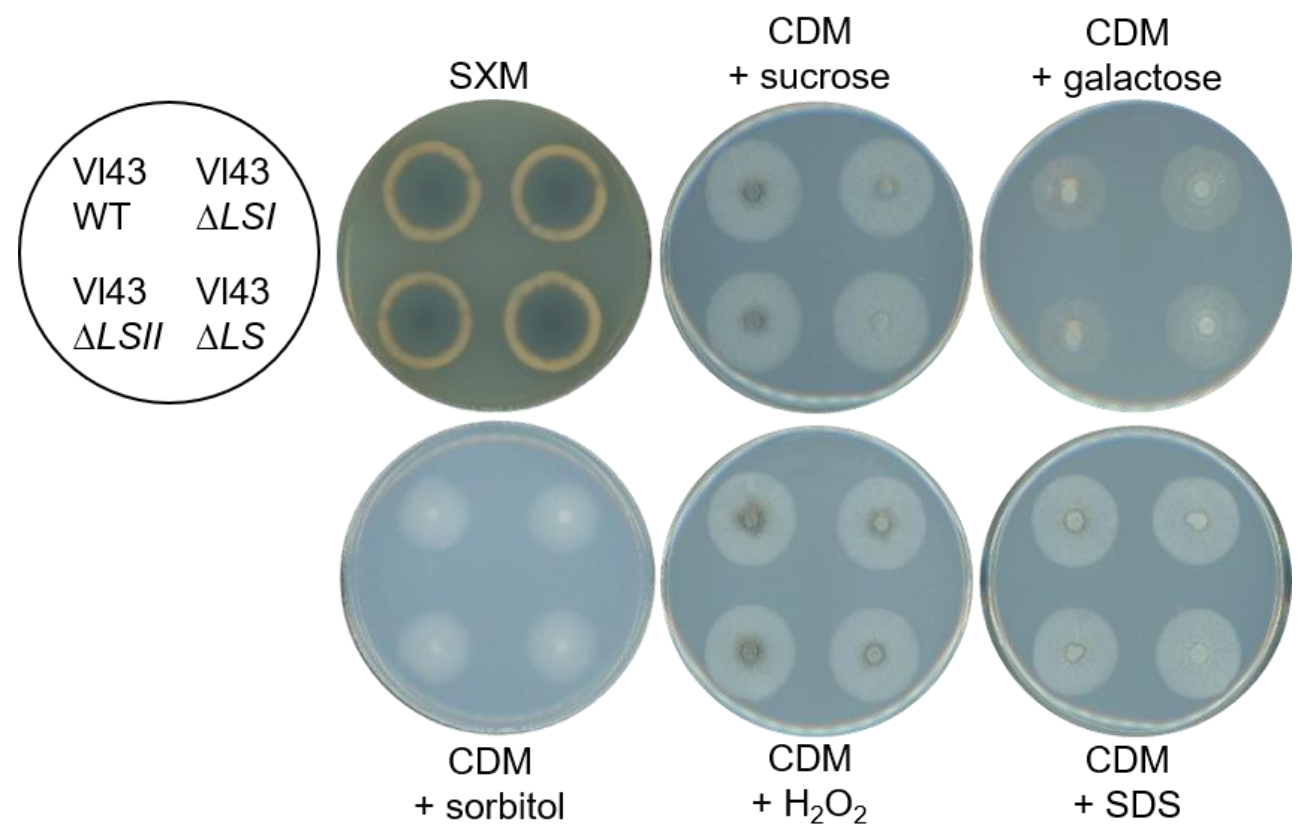

Figure S2: V. longisporum VI43LS20kb region is dispensable for vegetative growth on standard and stress inducing media ex planta. Ex planta phenotypes of VI43 LSI, LSII, and $L S$ deletion strains compared to wild type VI43 14 days after spot inoculation of 50000 spores on SXM, CDM with either sucrose or galactose as carbon source, or CDM supplemented with either $0.8 \mathrm{M}$ sorbitol, $0.00075 \% \mathrm{H}_{2} \mathrm{O}_{2}$, or $0.004 \%$ SDS incubated at $25^{\circ} \mathrm{C}$. Two independent transformants were tested for VI43 $\Delta L S I$ (VGB201, VGB233) and VI43 $\Delta L S I I$ (VGB193, VGB214), and a single transformant for VI43 $\Delta L S$ (VGB232). 
A

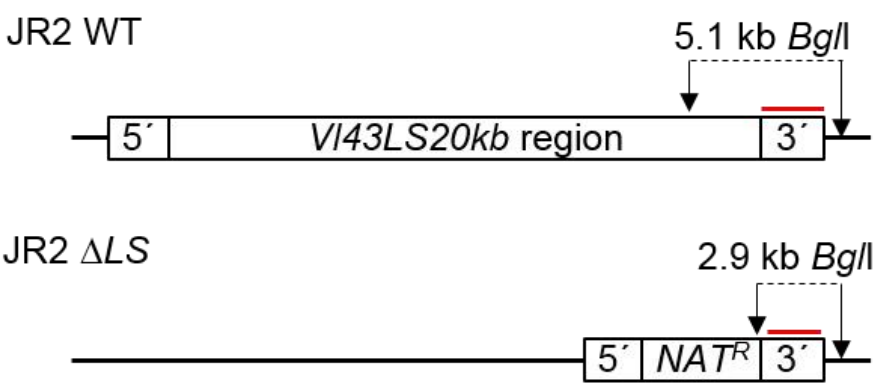

B

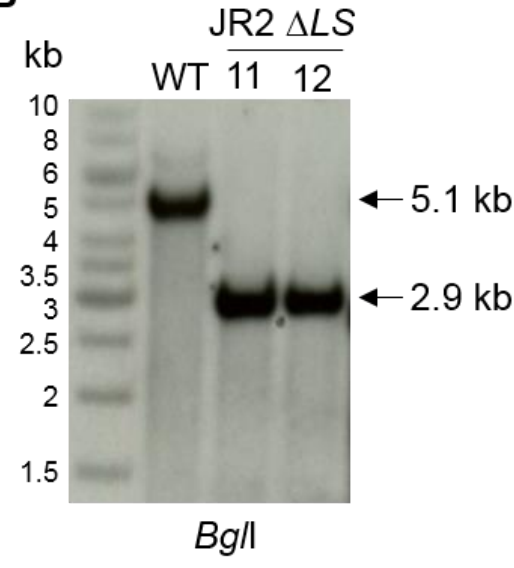

Figure S3: Southern hybridization of $V$. dahliae LS deletion transformants. (A) Scheme of restriction sites used for Southern hybridization. V. dahliae JR2 lacking the VI43LS20kb homologous region was constructed by replacement of the genomic region against a nourseothricin resistance marker cassettes under control of a gpdA promoter and a trpC terminator $\left(N A T^{R}\right)$ by homologous recombination of the up- and downstream flanking regions. Restriction sites of $B g / l$ are indicated by arrows and the expected fragment lengths labeled with the 3'-flanking region as a probe (indicated in red) are given. (B) Confirmation of two independent transformants of the V. dahliae JR2 LS deletion strain (VGB383, VGB393) by Southern hybridization using restriction enzyme $B g / l$ and $2.1 \mathrm{~kb} 3^{\prime}$-flanking region as a probe. Genomic DNA of the wild type was used as a control. The predicted bands of $5.1 \mathrm{~kb}$ for the wild type and $2.9 \mathrm{~kb}$ for the deletion were obtained. 


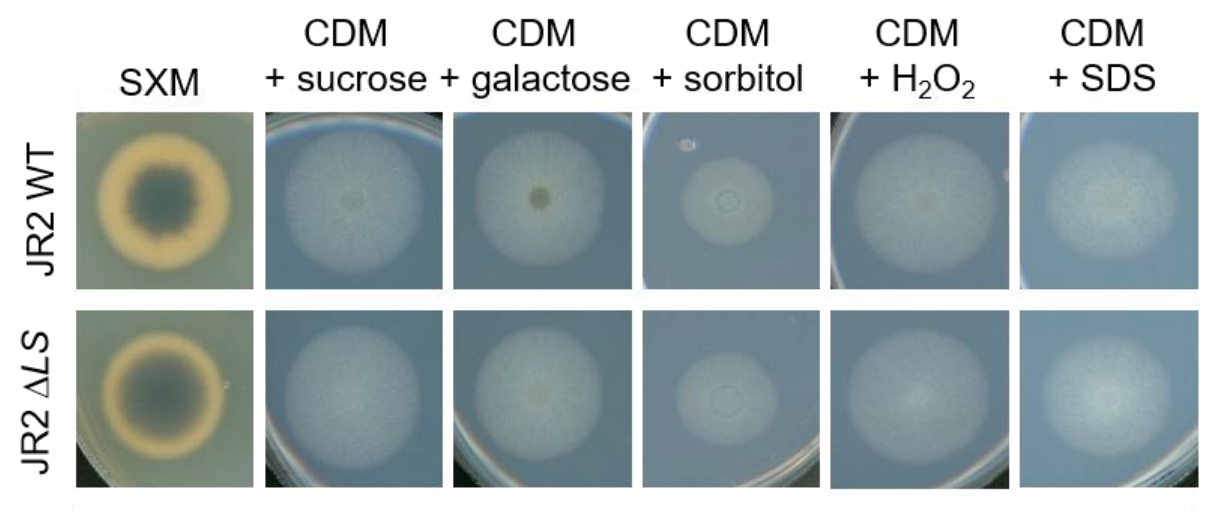

Figure S4: The $V$. dahliae JR2 LS deletion strain displays no significant alterations in its ex planta phenotype. Ex planta phenotypes of the $V$. dahliae JR2 LS deletion strain ten days after spot inoculation of 50000 spores on SXM, CDM with either sucrose or galactose as carbon source, or CDM supplemented with $0.8 \mathrm{M}$ sorbitol, $0.00075 \% \mathrm{H}_{2} \mathrm{O}_{2}$, or $0.004 \%$ SDS incubated at $25^{\circ} \mathrm{C}$. Two independent transformants were tested (VGB383, VGB393). 
A
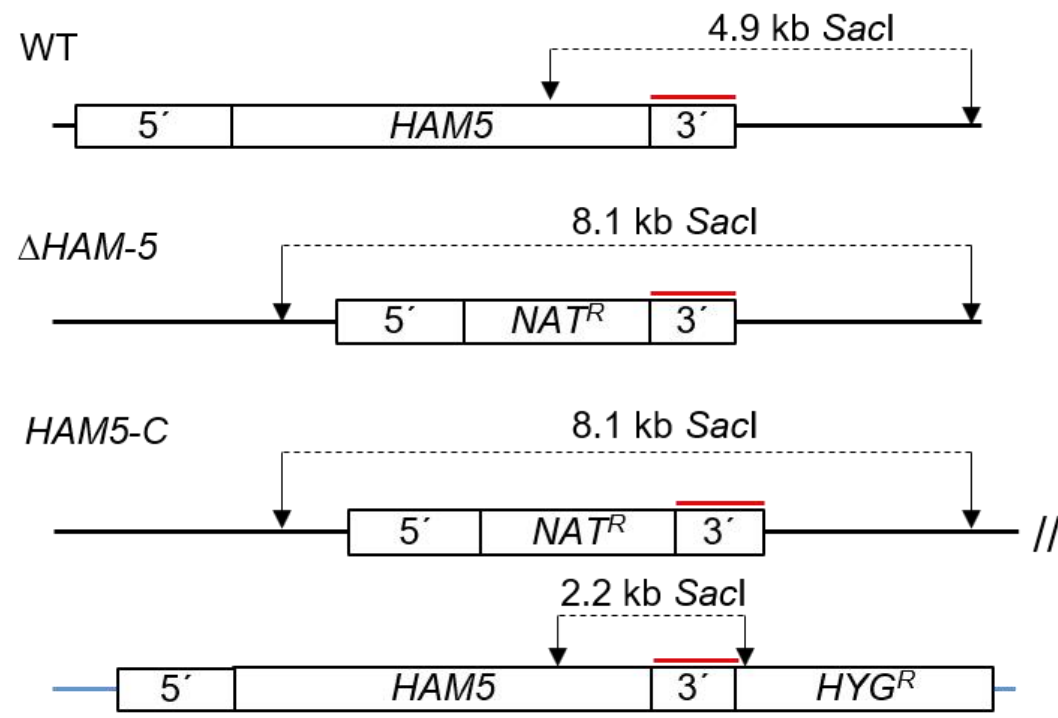

B

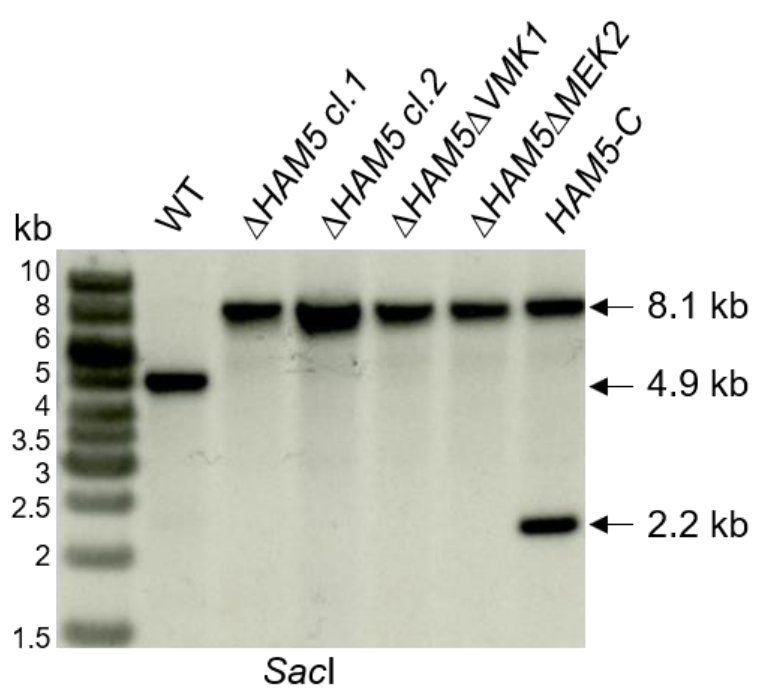

Figure S5: Southern hybridization of V. dahliae HAM5 single and VMK1/MEK2 double deletions and complementation strains. (A) Scheme of restriction sites used for Southern hybridization. $H Y G^{R}$ : the hygromycin resistance cassette under control of the gpdA promoter and a trpC terminator; $N A T^{R}$ : the nourseothricin resistance cassette under control of the gpdA promoter and a trpC terminator; restriction sites of Sacl are indicated by arrows and the expected fragment lengths labeled with the $3^{\prime}$-flanking region as a probe (indicated in red) are given. Ectopic integration sites of complementation strains are indicated by // at the end of the endogenous locus and by a blue line for the ectopic locus. (B) Southern hybridization for confirmation of strains: $\triangle H A M 5$ transformant number 1 (VGB279) and 2 (VGB280), $\triangle H A M 5 \triangle V M K 1$ (VGB417), $\triangle H A M 5 \triangle M E K 2$ (VGB346), HAM5-C (VGB415). Genomic DNA from wild type JR2 was used as a control. The $3^{\prime}$-flanking regions of HAM5 was used as a probe and genomic DNA was cut using the restriction enzyme Sacl. The predicted bands of $4.9 \mathrm{~kb}$ for wild type, $8.1 \mathrm{~kb}$ for the deletion, and $2.2 \mathrm{~kb}$ and $8.1 \mathrm{~kb}$ for the complementation were observed. 
A
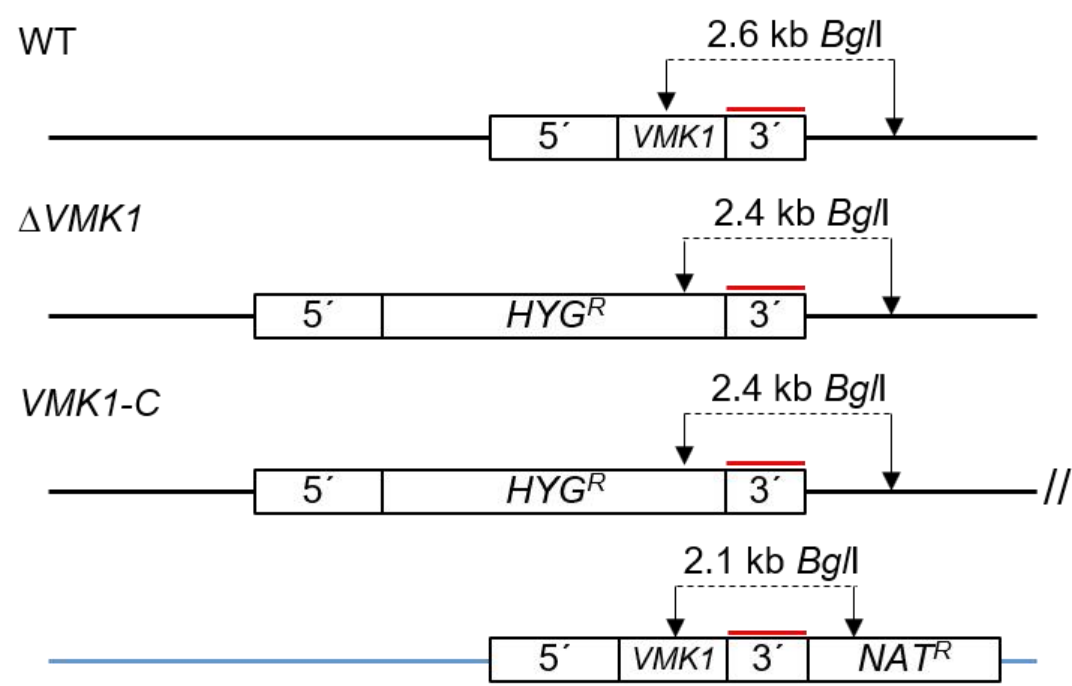

B

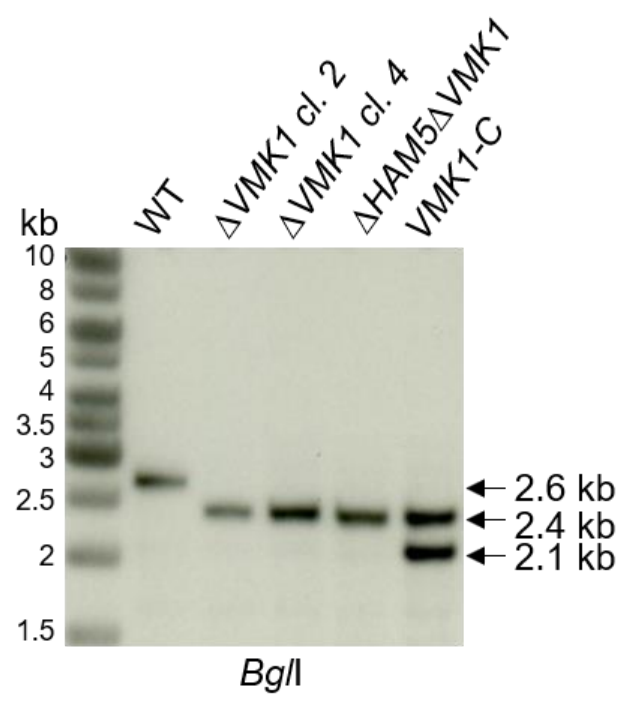

Figure S6: Southern hybridization of $V$. dahliae VMK1 single and HAM5/VMK1 double deletions and complementation strains. (A) Scheme of restriction sites used for Southern hybridization. $H Y G^{R}$ : the hygromycin resistance cassette under control of the gpdA promoter and a trpC terminator; $N A T^{R}$ : the nourseothricin resistance cassette under control of the gpdA promoter and a trpC terminator; restriction sites of $B g / l$ are indicated by arrows and the expected fragment lengths labeled with the $3^{\prime}$-flanking region as a probe (indicated in red) are given. Ectopic integration sites of complementation strains are indicated by // at the end of the endogenous locus and by a blue line for the ectopic locus. (B) Southern hybridization for confirmation of strains: $\triangle V M K 1$ transformant number 2 (VGB335) and 4 (VGB336), $\triangle H A M 5 \triangle V M K 1$ (VGB417), VMK1-C (VGB413). Genomic DNA was used from wild type JR2 as a control. The $3^{\prime}$-flanking region of VMK1 was used as a probe and genomic DNA was cut using the restriction enzyme $\mathrm{Bgll}$. The predicted bands of $2.6 \mathrm{~kb}$ for wild type, $2.4 \mathrm{~kb}$ for the deletion, and $2.1 \mathrm{~kb}$ and $2.4 \mathrm{~kb}$ for the complementation were observed. 
A

WT

$5.2 \mathrm{~kb} \mathrm{Nrul}$

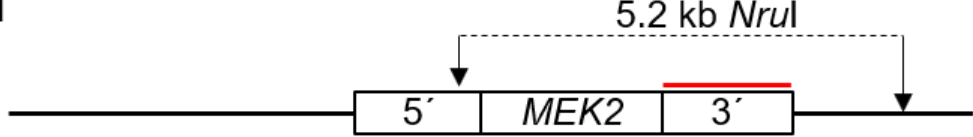

$\triangle M E K 2$

$7.1 \mathrm{~kb}$ Nrul

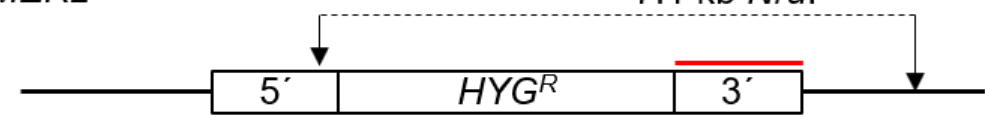

MEK2-C

$7.1 \mathrm{~kb}$ Nrul
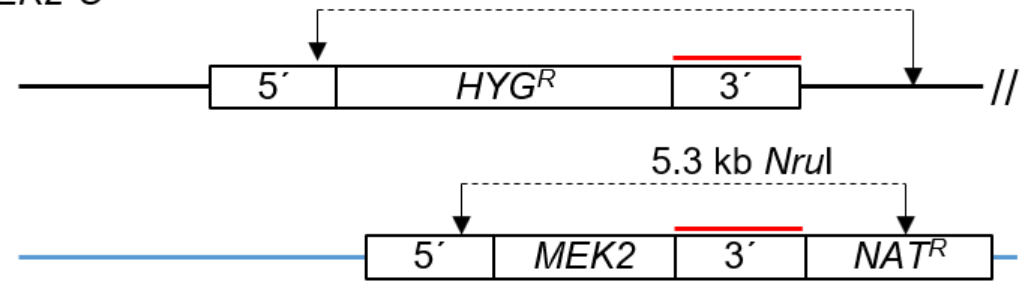

B

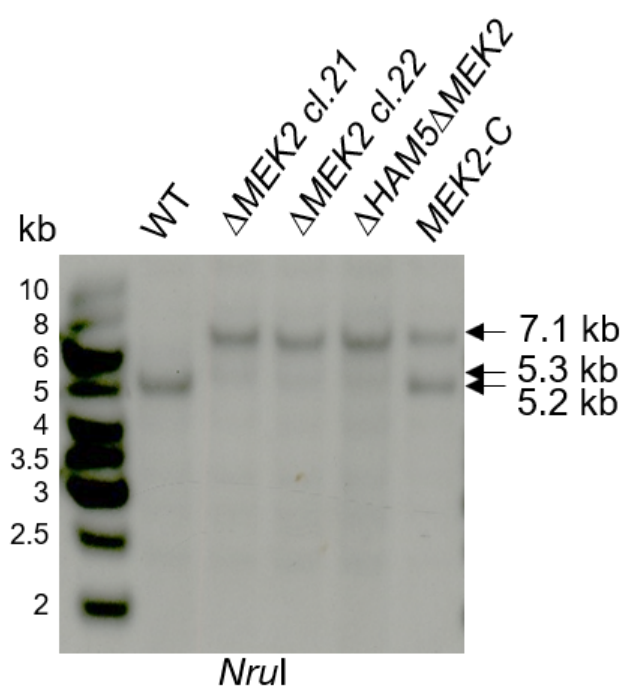

Figure S7: Southern hybridization of $V$. dahliae MEK2 single and HAM5/MEK2 double deletions and complementation strains. (A) Scheme of restriction sites used for Southern hybridization. $H Y G^{R}$ : the hygromycin resistance cassette under control of the gpdA promoter and a $\operatorname{trpC}$ terminator; $N A T^{R}$ : the nourseothricin resistance cassette under control of the gpdA promoter and a trpC terminator; restriction sites of $\mathrm{Nrul}$ are indicated by arrows and the expected fragment length labeled with the $3^{\prime}$-flanking region as a probe (indicated in red) are given. Ectopic integration sites of complementation strains are indicated by // at the end of the endogenous locus and by a blue line for the ectopic locus. (B) Southern hybridization for confirmation of strains: $\triangle M E K 2$ transformant number 21 (VGB337) and 22 (VGB338), $\triangle H A M 5 \triangle M E K 2$ (VGB346), and MEK2-C (VGB389). Genomic DNA was used from wild type JR2 as a control. The $3^{\prime}$-flanking region of MEK2 was used as a probe and genomic DNA was cut using the restriction enzyme Nrul. The predicted bands of $5.2 \mathrm{~kb}$ for WT, $7.1 \mathrm{~kb}$ for the deletion, and $5.3 \mathrm{~kb}$ and $7.1 \mathrm{~kb}$ for the complementation were observed. 
A
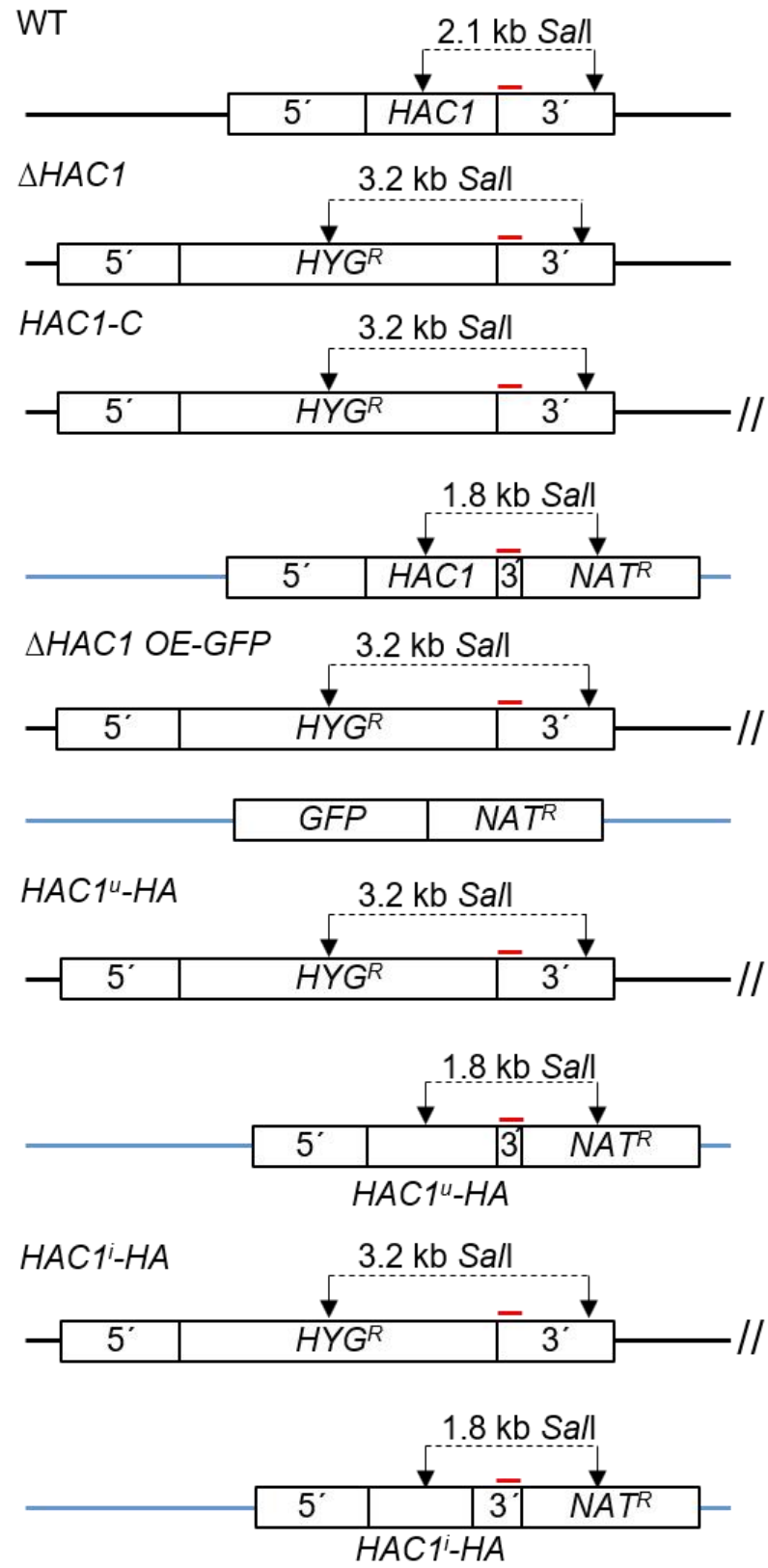

Figure S8: Southern hybridization of V. dahliae HAC1 deletion, HAC1 deletion with ectopic GFP overexpression, HAC1 complementation, as well as $H A C 1^{u_{-}} H A$ and HAC1 ${ }^{i}-H A$ strains. (A) Scheme of restriction sites used for Southern hybridization. $H Y G^{R}$ : the hygromycin resistance cassette under control of the gpdA promoter and a trpC terminator; $N A T^{R}$ : the nourseothricin resistance cassette under control of the gpdA promoter and a $\operatorname{trp} C$ terminator; restriction sites of Sall are indicated by arrows. Expected fragment lengths labeled with the 3'-flanking region as a probe (indicated in red) are given. Ectopic integration sites of complementation strains are indicated by // at the end of the endogenous locus and by a blue line for the ectopic locus. (B) Southern hybridization for confirmation of strains: $\triangle H A C 1$ transformant number 1 (VGB371) and 23 (VGB372), $\triangle H A C 1$ OE-GFP (VGB380), HAC1-C complementation (VGB382), HAC14-HA transformant number 2 (VGB439) and 5 (VGB440), and HAC1'-HA transformant number 6 (VGB437) and 10 (VGB438). The 3'-flanking region of $H A C 1$ was used as a probe. Genomic DNA was cut using the restriction enzyme Sall. The predicted bands of $2.1 \mathrm{~kb}$ for wild type, $3.2 \mathrm{~kb}$ for $\triangle H A C 1$ and $\triangle H A C 1 O E-G F P$, and $3.2 \mathrm{~kb}$ and $1.8 \mathrm{~kb}$ for the HAC1-C, HAC1 ${ }^{u}-H A$, and $H A C 1^{i}-H A$ strains were obtained. 
A
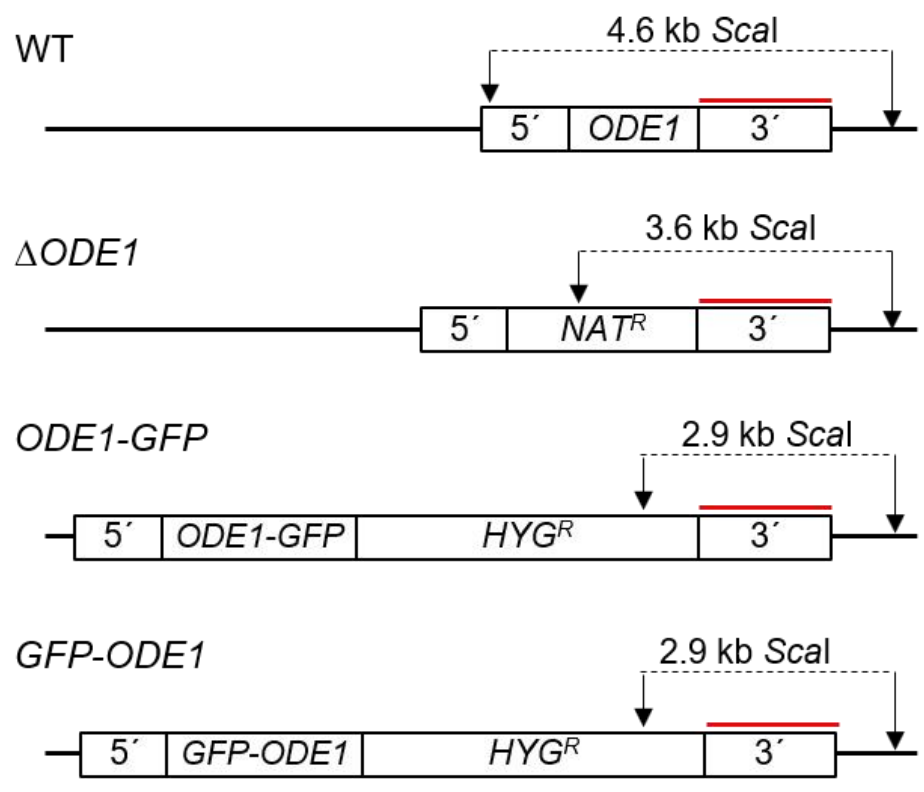

B

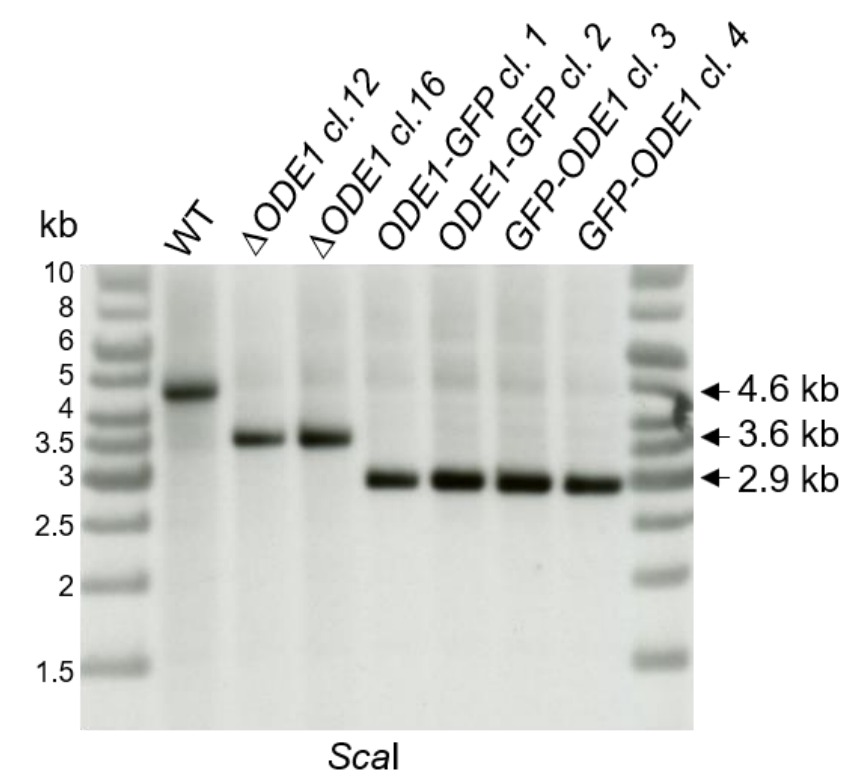

Figure S9: Southern hybridization of $V$. dahliae ODE1 deletion and complementation strains. (A) Scheme of restriction sites used for Southern hybridization. $H Y G^{R}$ : the hygromycin resistance cassette under control of the $g p d A$ promoter and a $\operatorname{trp} C$ terminator; $N A T^{R:}$ : the nourseothricin resistance cassette under control of the gpdA promoter and a $\operatorname{trpC}$ terminator; restriction sites of Scal are indicated by arrows and the expected fragment lengths labeled with the $3^{\prime}$-flanking region as a probe (indicated in red) are given. (B) Southern hybridization for confirmation of strains: $\triangle O D E 1$ transformant number 12 (VGB331) and 16 (VGB332), ODE1-GFP transformant number 1 (VGB358) and 2 (VGB359), and GFP-ODE1 transformant number 3 (VGB360) and 4 (VGB361). The 3'-flanking region of ODE1 was used as a probe. Genomic DNA was cut using the restriction enzyme Scal. The predicted bands of $4.6 \mathrm{~kb}$ for wild type, $3.6 \mathrm{~kb}$ for the deletion, and $2.9 \mathrm{~kb}$ for the endogenous complementation strains were observed. 
ATG GAG TCT TGG GAG CAC TCC ACC ACA CCA ATG ATC AAG TTC GAG GAC TCG CCA GCC GAG TCT TTC GTC TCG ACA CCA GGC GAC ATG TAC CCG TCA CTC TTC CCA GAG TCC GCC TCC CCC AAC ACC CTC GAT CCT TCC AAC ATG ATG AGC CCT TCC TCA CCC CAA GAC CTC ACC ATT GCC GAC ACG GAT ATG CCT CTC TCC GAG GCT TCC GCC GGC GAC AAG AAG GGG TCC AAG AAG CGC AAG TCC TGG GGT CAG GTC CTT CCC GAG CCC AAG ACC AAC TTG CCG CCC AGG AAA CGA GCC AAG ACT GAG GAT GAG AAG GAG CAG CGT CGT GTG GAA CGC GTT CTG CGC AAC CGC CGT GCT GCC CAG TCT TCG AGG GAG CGC AAG AGG CTC GAG GTT GAG GCC CTC GAG ATG AAG AAC AAG GAG CTC GAG ACT GCC CTG AAC CAC GCA CAA CAG GCG AAC GCT AGG TTG ATG GAG GAG CTT ACC AAG TTC CGC CGT GGT TCC GGT GCC GTC GCC CGT TCT TCT TCC CCC TTT GAC TCC TTC CAC AAC AGC AAC TCG GTC ACC CTC TCC CCC GAG CTG TTC GGC TCT CAA GAC GGC CGC CGG CCA TCA GTG GCC GAC TCC GAG TCG ACA CTC GTC GAC GGT TTG ATG GCG GCC TCC AAG TCC GCC GCG ACC GTC AAC CCC GCC TCC CTC TCG CCC GCC CTC ACC CCC GTC CCC GAG ACG GAT GAG ACC AGC GCC CAA CAA GAA GCT GCC GTG GCC GCC CCT TCC CCT GTC GCC CTT TCC TCC GAC GTG ACA CAA CGT CCT GCC GTG TCG GTC GGA GGA AAT GCC TCA GTC GTG GGT GGC CTC GCA GAC TTC CCT GCA CCC AAC ATG GAC TTT GTA CCT TCA GCT TCA GAT GCT CAT GAT CAC TTC CTC GGC GGT CAT TTC AGC GTG TCA GAG GCC TTT GAT GCA GAT CGC TAT GTC CTT GAG AGC GGG CTT CTC TCT TCC CCC AAC TCA GTC GAT TAT GAC AAC GAT ATT ATG GCT GGT GAC TCG TCC GCG TTC GCA TCC GCG TTC AAC TTC GAC ATG GAC GAG TTC CTC AAC GAT GAG GCC AGC GCA GCC GCC ACT GAC GCG TCA GCA GCG GAG AAC AGC GCA GCG GAC CCG GAC TAC GGC CGC CGT GCC CTT AAC CCT GAG ACT CAA GTC TCT TCA GAA AAT CCT AAC CTG CAG CCC CAA TCT GGC GCG TCC ACT TAT GGA TGC GAC GAT GGA GGC ATT GCG GTT GGT GTC TGA
30
60
90
120
150
180
210
240
270
300
330
360
390
420
450
480
510
540
570
600
630
660
690
720
750
780
810
840
870
900
930
960
990
1020
1050
1080
1110
1140
1170
1200
1230
1254

Figure S10: The cDNA sequence of $V$. dahliae JR2 HAC1'. The V. dahliae JR2 HAC1' sequence with 1254 nucleotides was obtained by cDNA synthesis from mycelium of wild type

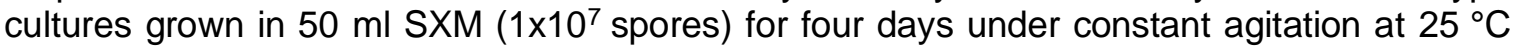
and subsequent supplementation with $3 \mathrm{mM}$ DTT for three hours. HAC1 ${ }^{i}$ was amplified using primers JST171/JST174 and was fully sequenced. 


$\begin{array}{rllllr}1 & \text { MESWEHSTTP } & \text { MIKFEDSPAE } & \text { SFVSTPGDMY } & \text { PSLFPESASP } & 40 \\ 41 & \text { NTLDPSNMMS } & \text { PSSPQDLTIA } & \text { DTDMPLSEAS } & \text { AGDKKGSKKR } & 80 \\ 81 & \text { KSWGQVLPEP } & \text { KTNLPPRKRA } & \text { KTEDEKEQRR } & \text { VERVLRNRRA } & 120 \\ 121 & \text { AQSSRERKRL } & \text { EVEALEMKNK } & \text { ELETALNHAQ } & \text { QANARLMEEL } & 160 \\ 161 & \text { TKERRGSGAV } & \text { ARSSSPFDSF } & \text { HNSNSVTLSP } & \text { ELFGSQDGRR } & 200 \\ 201 & \text { PSVADSESTL } & \text { VDGLMAASKS } & \text { AATVNPASLS } & \text { PALTPVPETD } & 240 \\ 241 & \text { ETSAQQEAAV } & \text { AAPSPVALSS } & \text { DVTQRPAVSV } & \text { GGNASVVGGL } & 280 \\ 281 & \text { ADFPAPNMDF } & \text { VPSASDAHDH } & \text { FLGGHFSVSE } & \text { AFDADRYVLE } & 320 \\ 321 & \text { SGLLSSPNSV } & \text { DYDNDIMAGD } & \text { SSAFASAFNF } & \text { DMDEFLNDEA } & 360 \\ 361 & \text { SAAATDASAA } & \text { ENSAADPDYG } & \text { RRALNPETQV } & \text { SSENPNLQPQ } & 400 \\ 401 & \text { SGASTYGCDD } & \text { GGIAVGV } & & & \end{array}$

Figure S11: The amino acid sequence of $V$. dahliae JR2 Hac1. The deduced protein sequence from $H A C 1^{i}$ with 417 aa in length. Red: NLS predicted by cNLS Mapper (94-105 aa); Blue: N-terminal basic-leucine zipper domain (bZIP, PS50217; 107-164 aa). 


\section{List of Figures}

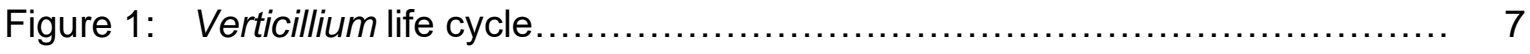

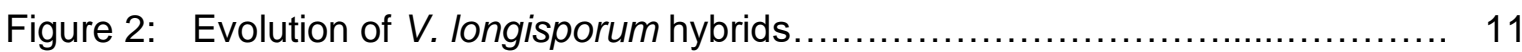

Figure 3: The yeast Fus3 and Kss1 MAPK pathways and homologous cascades in filamentous ascomycetes $A$. nidulans and $V$. dahliae.......................... 14

Figure 4: Hac1 is the central unfolded protein response regulator in S. cerevisiae..... 18

Figure 5: Linoleic acid biosynthesis by oleate $\Delta 12$-fatty acid desaturases............. 22

Figure 6: Fungal and plant oxylipins in crosstalk and fungal differentiation.............. 25

Figure 7: $\quad$ V. longisporum strains VI43 and VI32 colonize B. napus plants either pathogenically or asymptomatically

Figure 8: The symptomatic $V$. longisporum strain V143 harbors a specific VI43LS20kb region in its genome, which is absent in the asymptomatic isolate $\mathrm{VI} 32$.

Figure 9: The VI43LS2Okb region absent in the asymptomatic $V$. longisporum isolate VI32 attenuates disease symptom induction of VI43 in B. napus.

Figure 10: The VI43LS20kb homologous region in $V$. dahliae is indifferent for virulence towards tomato plants

Figure 11: Comparison of $V$. dahliae Ham5 and corresponding proteins in related fungi.

Figure 12: The $V$. dahliae MAP2K Mek2 and MAPK Vmk1 are highly conserved in fungi.

Figure 13: V. dahliae VMK1- and MEK2-mediated vegetative growth and microsclerotia formation function independent of HAM5

Figure 14: V. dahliae Vmk1 pathway-mediated pathogenicity towards S. Iycopersicum is independent from the scaffold protein.

Figure 15: Protein domains of $V$. dahliae Ire1 and Hac1 ${ }^{\mathrm{u}}$ in comparison to corresponding proteins of related filamentous fungi

Figure 16: The $V$. dahliae HAC1 mRNA is unconventionally spliced. 78

Figure 17: Similarities of Hac1 proteins from different organisms.

Figure 18: The $V$. dahliae unconventionally spliced mRNA HAC1', but not the uninduced mRNA HAC1" , is translated into the Hac1 protein.

Figure 19: $V$. dahliae requires the expression of $H A C 1$ for growth with or without tunicamycin-induced ER stress and melanization.

Figure 20: $V$. dahliae HAC1 is essential for microsclerotia formation.

Figure 21: $V$. dahliae HAC1 positively regulates conidiospore formation

Figure 22: $V$. dahliae $H A C 1$ is involved in initial colonization but dispensable for penetration of $A$. thaliana roots.

Figure 23: $V$. dahliae HAC1 is required for induction of disease symptoms in S. lycopersicum

Figure 24: $V$. dahliae $O D E 1$ encodes a membrane-bound oleate $\Delta 12$-fatty acid desaturase.

Figure 25: $V$. dahliae Ode1 is localized to plasma and organelle membranes different to vacuoles

Figure 26: $V$. dahliae $O D E 1$ contributes to vegetative growth.

Figure 27: $V$. dahliae $O D E 1$ is involved in microsclerotia formation. 
Figure 28: $V$. dahliae $O D E 1$ is not required for induction of severe disease symptoms in S. lycopersicum plants.

Figure 29: Interactions between haploid $V$. dahliae and amphidiploid $V$. longisporum isolates with their plant hosts studied in this thesis.

Figure 30: The VI43LS20kb region attenuates pathogenicity of $V$. longisporum VI43 in rapeseed and the homologousregion is indifferent for virulence of V. dahliae JR2 on tomato plants

Figure 31: MAPK signaling pathways in $V$. dahliae................................. 110

Figure 32: $V$. dahliae HAC1 regulates differentiation and virulence........................ 120

Figure 33: $V$. dahliae Ode1 displays a strong impact on vegetative growth, but is dispensable for induction of disease symptoms.... 123 


\section{List of Tables}

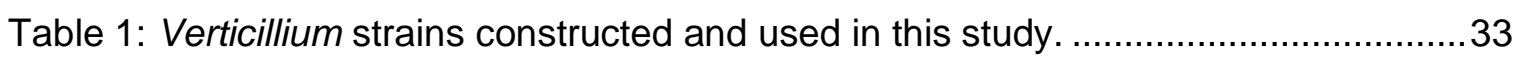

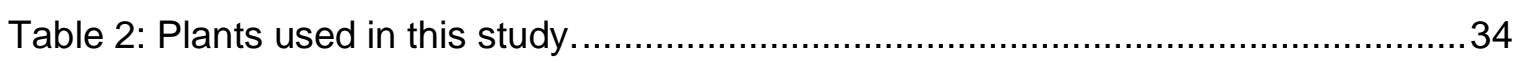

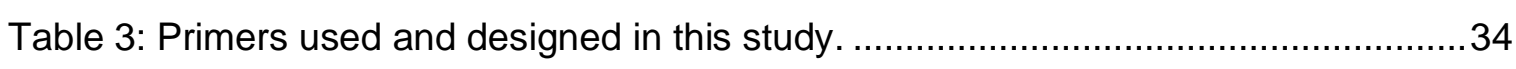

Table 4: Plasmids constructed and used in this study ............................................. 39 


\section{List of Supplementary Figures}

Figure S1: Southern hybridization of V. Iongisporum VI43 LSI, LSII, and LS deletion strains.

Figure S2: $\quad$. longisporum VI43LS20kb region is dispensable for vegetative growth on standard and stress inducing media ex planta.

Figure S3: Southern hybridization of $V$. dahliae LS deletion transformants

Figure S4: The $V$. dahliae JR2 LS deletion strain displays no significant alterations in its ex planta phenotype.

Figure S5: Southern hybridization of $V$. dahliae HAM5 single and VMK1/MEK2 double deletions and complementation strains

Figure S6: Southern hybridization of $V$. dahliae VMK1 single and HAM5/VMK1 double deletions and complementation strains....

Figure S7: Southern hybridization of $V$. dahliae MEK2 single and HAM5/MEK2 double deletions and complementation strains

Figure S8: Southern hybridization of $V$. dahliae HAC1 deletion, HAC1 deletion with ectopic GFP overexpression, HAC1 complementation, as well as $H A C 1^{u}-H A$ and $H A C 1^{u}-H A$ strains.

Figure S9: Southern hybridization of $V$. dahliae ODE1 deletion and complementation strains

Figure S10: The cDNA sequence of $V$. dahliae JR2 HAC1 ${ }^{i}$. 154

Figure S11: The amino acid sequence of $V$. dahliae JR2 Hac1 155 


\section{Abbreviations}

5

$3^{\prime}$

12-OH-JA

$\alpha$

${ }^{\circ} \mathrm{C}$

$\Delta$

$\lambda$

$\mu \mathrm{g}$

$\mu \mathrm{l}$

$\mu \mathrm{m}$

$\mu \mathrm{mol}$

aa

$A M P^{R}$

Amr

APS

AS

ATF

ATMT

bHLH

BLAST

bp

bZIP

CDM

cDNA

Cib

cl.

$\mathrm{cm}$

$\mathrm{COI}$

C-terminus

CWDE(s)

CWI

DIC

DNA

DTT

ECL

EDTA

ER

ERAD

et al.

$\mathrm{EtOH}$

FAD

FLO

FM4-64

Fus

$g$

GFP

$\mathrm{GmbH}$

${ }^{\circ}$ gpdA

G-protein upstream flanking region

downstream flanking region

12-hydroxy jasmonic acid

antibody

degree Celsius

deletion

wavelength

microgram

microliter

micrometer

micromol

amino acid(s)

ampicillin resistance marker cassette

Alternaria melanin regulation

ammonium persulfate

acetosyringone

activating transcription factor

Agrobacterium tumefaciens-mediated transformation

basic helix-loop-helix

basic local alignment search tool

base pair(s)

basic leucine zipper

Czapek-Dox medium

complementary deoxyribonucleic acid

Clp1 interacting bZIP1

clone/ transformant

centimeter(s)

coronatine insensitive

carboxy terminus

cell wall degrading enzyme(s)

cell wall integrity

differential interference contrast

deoxyribonucleic acid

dithiothreitol

enhanced chemiluminescence

$2,2^{\prime}, 2^{\prime \prime}, 2^{\prime \prime \prime}$-(Ethane-1,2-diyldinitrilo) tetra-acetic acid

endoplasmic reticulum

endoplasmic reticulum associated protein degradation

et alii (and others)

ethanol

fatty acid desaturase

flocculation

$\mathrm{N}$-(3-Triethylammoniumpropyl)-4-(6-(4-(Diethylamino) Phenyl)

Hexatrienyl) Pyridinium Dibromide

cell fusion

gram

green fluorescent protein

Gesellschaft mit beschränkter Haftung

A. nidulans glyceraldehyde-3-phosphate dehydrogenase promoter

guanine nucleotide-binding protein 


\begin{tabular}{|c|c|}
\hline $\mathrm{H}_{2} \mathrm{O}_{2}$ & hydrogen peroxide \\
\hline HA & hemagglutinin \\
\hline $\mathrm{Hac}$ & $\begin{array}{l}\text { homologous to Atf/Creb (activating transcription factor/ cAMP } \\
\text { response element-binding protein) }\end{array}$ \\
\hline$H A C 1-C$ & HAC1 complementation \\
\hline$H A C 1^{i}$ & induced messenger RNA variant of $H A C 1$ \\
\hline$H A C 1^{i}-H A$ & $\begin{array}{l}\text { induced messenger RNA variant of } H A C 1 \text { with } 5^{\prime} \\
\text { hemagglutinin sequence }\end{array}$ \\
\hline$H A C 1^{u}$ & uninduced messenger RNA variant of $H A C 1$ \\
\hline$H A C 1^{u}-H A$ & $\begin{array}{l}\text { uninduced messenger RNA variant of } H A C 1 \text { with } 5^{\prime} \\
\text { hemagglutinin sequence }\end{array}$ \\
\hline Ham & hyphal anastomosis mutant \\
\hline HAM5-C & HAM5 complementation \\
\hline Hog & high osmolarity glycerol \\
\hline HRP & horseradish peroxidase \\
\hline $\mathrm{Hxl}$ & HAC1 and XBP1-like gene \\
\hline Ire & inositol requiring \\
\hline IRTG & international research training group \\
\hline JA & jasmonic acid \\
\hline JA-Ile & jasmonoyl isoleucine \\
\hline JAZ & jasmonate ZIM-domain \\
\hline$K A N^{R}$ & kanamycin resistance marker cassette \\
\hline $\mathrm{kb}$ & kilobase(s) \\
\hline kDA & kilo Dalton \\
\hline KGaA & Kommanditgesellschaft auf Aktien \\
\hline Kpp & kinase PCR-product \\
\hline Kss & kinase suppressor of Sst2 (super sensitive 2) mutations \\
\hline I & liter \\
\hline LA & linoleic acid \\
\hline LasA & JA ester lasiojasmonate $A$ \\
\hline LB & lysogeny broth \\
\hline LDS(s) & linoleate diol synthase(s) \\
\hline LOX(s) & lipoxygenase (s) \\
\hline LS & lineage specific \\
\hline$L S G(s)$ & lineage specific region gene(s) \\
\hline $\mathrm{M}$ & molar \\
\hline MAK & mitogen activated kinase \\
\hline MAPK & mitogen activated protein kinase(s) \\
\hline MEK & MAPK/ERK kinase \\
\hline MEK2-C & MEK2 complementation \\
\hline MES & 2-(N-Morpholino)-ethane sulphonic acid monohydrate \\
\hline $\mathrm{Mb}$ & mega base pair(s) \\
\hline $\mathrm{mg}$ & milli gram(s) \\
\hline $\min$ & minute(s) \\
\hline $\mathrm{ml}$ & milliliter(s) \\
\hline $\mathrm{mm}$ & millimeter(s) \\
\hline $\mathrm{mM}$ & millimolar \\
\hline mRNA & messenger RNA \\
\hline $\mathrm{ms}$ & millisecond(s) \\
\hline Msb & multicopy suppression of a budding defect \\
\hline Mw & molecular weight \\
\hline & number of elements \\
\hline$N A T^{R}$ & nourseothricin resistance marker cassette \\
\hline
\end{tabular}




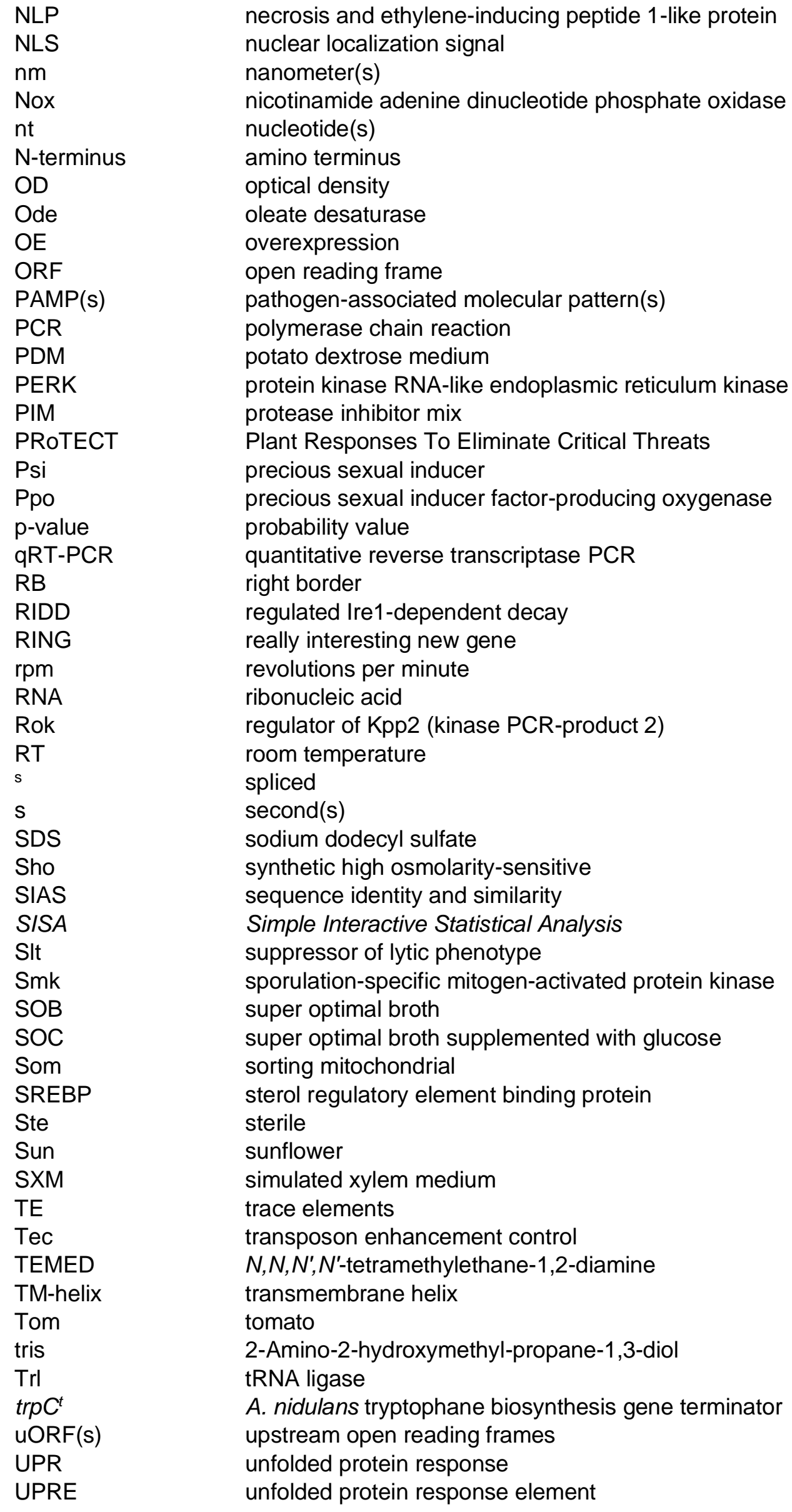




$\begin{array}{ll}\text { UTR } & \text { untranslated region } \\ \text { UV } & \text { ultra-violet } \\ \text { V } & \text { volt } \\ \text { Va } & \text { Verticillium alfalfae } \\ \text { Vayg } & \text { Verticillium homolog to Aspergillus yellowish green } \\ \text { Vd } & \text { Verticillium dahliae } \\ \text { VdCmr } & \text { V. dahliae homolog to Colletotrichum melanin regulation } \\ \text { Ve/Vel } & \text { velvet } \\ \text { VGB } & \text { Verticillium strain collection Gerhard H. Braus } \\ \text { VI43LS20kb } & \text { Verticillium longisporum 43 approximately } 20 \text { kb lineage specific } \\ \text { VI } & \text { Verticillium longisporum } \\ \text { Vmk } & \text { Verticillium mitogen activated protein kinase } \\ \text { VMK1-C } & \text { VMK1 complementation } \\ \text { WD40 } & \text { tryptophan-aspartic acid dipeptide ending structural motif of } \\ \text { WT } & \text { circa 40 amino acids } \\ \text { XBP } & \text { wild type } \\ & \text { X-box binding protein }\end{array}$




\section{Acknowledgements}

Foremost, I would like to thank Prof. Dr. Gerhard H. Braus for his support, fruitful discussions, his expertise and helpful advice during my Ph.D. project. I am grateful for the opportunity to join his working group, national and international scientific meetings, and the international research training group (IRTG) 2172 "Plant Responses To Eliminate Critical Threats" (PRoTECT) with an active exchange to the cooperating research groups at the University of British Columbia in Vancouver. I am thankful to our cooperation partner and member of the thesis advisory committee Prof. Ph.D, James W. Kronstad for his commitment and hospitality during my stay in his group and for sharing his expertise in the field of fungal pathogenesis. I want to thank apl. Prof. Dr. Kai Heimel for being a member of my thesis committee. I sincerely appreciate his support, his expertise in the research field of the unfolded protein response in plant-fungal interactions, a lot of helpful advice, and motivating suggestions.

Additionally, I thank Prof. Dr. Andrea Polle, Prof. Dr. Petr Karlovsky, and Prof. Dr. Volker Lipka for being members of my examination board and for fruitful discussions in frame of the IRTG 2172 PRoTECT meetings.

I want to thank the IRTG speaker Prof. Dr. Ivo Feußner, all principle investigators from the University of Goettingen and the University of British Columbia, and my fellow students. I am very grateful to be a part of the doctoral program IRTG 2172 PRoTECT of the Goettingen Graduate School for Neuroscience, Biophysics and Molecular Biosciences (GGNB). The GGNB allowed me to attend method and soft skill courses and gave me the financial support to visit the $12^{\text {th }}$ International Verticillium Symposium in Slowenia. The IRTG 2172 PRoTECT offered enrolled students to invite experts of the field plant-microbe interactions to seminars, to develop important competences in a broad selection of method and soft skill courses, and the opportunity to organize the International Plant Immunity Symposium (IPIS) 2018. Further, the IRTG 2172 PRoTECT made the cooperation with the University of British Columbia possible to get international experience and build up an international network. I had the chance to organize and participate at industry excursions to companies, from which I learned a lot for my Ph.D. and future career. This was facilitated by a great organizational support by the scientific and administrative coordinators Dr. Stefanie König, Judith Wassiltschenko and Anja Vogelpohl.

I thank Rebekka Harting, Nicole Scheiter, Miriam Leonard, Annalena Höfer, Dr. Tri-Thuc Bui, and the newly enrolled members of the Verticillium group Alexandra Nagel and Isabel Maurus. I enjoy the working atmosphere in our group, the discussions, the breaks and the self-evident commitment to mutual support. I am very grateful for the supervision 
of Dr. Rebekka Harting during my Ph.D. project. I appreciate our constructive discussions, her expertise, her advice regarding the project and the thesis, and her experimental support. I am grateful to Nicole for her kindness, organizational skills, and excellent technical support. My gratitude goes to Miriam for the experiences we made together in our Master and Ph.D. programs, in the working groups in Vancouver, and Goettingen and in our friendship. I can always count on her support and her organizational skills. Thanks to Annalena for her support, cheerfulness and humor. I want to thank Thuc for sharing his experimental experiences and for having a nice time together as lab neighbors. I am very grateful to Alexandra and Isabel for their great assistance in the final phase of my Ph.D. and for their diligence and friendliness and I am happy that they joined our group.

Furthermore, I want to thank Rica Bremenkamp, Jan Teer, and Eva Friederike Hettwer. Rica and Eva supported my project in lab rotations and proceeded as research assistants. Jan helped as a research assistant and went on, working on parts of my project during his Master thesis.

For the proofreading of my thesis I would like to thank Rebekka, Anna Maria Köhler, Annalena, Fruzsina Bakti, Miriam, and Alexandra.

Thanks to Dr. Ellen Hornung from the "Plant Biochemistry" for the provision of linoleic acid used in this study.

I am very grateful to Dr. Daniela Nordzieke who shared her protocols and expertise regarding hyphal and conidial anastomosis and supported me by searching for those in Verticillium.

I am very thankful to all members of the department of "Molecular Microbiology and Genetics" for a nice atmosphere, their kindness and helpfulness in everyday lab life. A special thank you to Heidi Northemann, Andrea Wäge, Katharina Ziese-Kubon, Gabriele Heinrich, and Verena Große for supporting administrative issues as well as for keeping everything going. Thanks as well, to the technical support provided by the maintenance facility.

My gratitude further goes to all lab members of the Kronstad lab in Vancouver who hosted Miriam and me during our stay. Special thanks to Sean, and Linda, who participated in the exchange of the IRTG and became friends.

Besides my colleagues, I would like to express my thanks to my friends from outside of the lab, my family, and Marcel for all the support and motivation they have given me. 